\title{
Análise experimental de algoritmos de planaridade
}

\author{
Alexandre Noma \\ DISSERTAÇÃO APRESENTADA AO \\ InSTITUTO DE MATEMÁTICA E \\ ESTATÍSTICA DA \\ Universidade de SÃo Paulo \\ COMO PARTE DOS REQUISITOS \\ PARA OBTENÇÃO DO GRAU DE \\ Mestre EM CiÊnCia da COMPUTAÇÃo
}

Orientadora: Profa. Dra. Cristina Gomes Fernandes.

- São Paulo, Maio de 2003 -

- Durante o desenvolvimento deste trabalho, o aluno recebeu apoio financeiro da FAPESP- 
Aos meus pais Massagi e Eliana. Aos meus irmãos Eduardo e Beatriz. A todos meus amigos ... ...e à Ayumi, com muito amor. 


\section{Agradecimentos}

Agradeço a Deus por me conceder uma família tão amorosa e pela oportunidade de estudar numa faculdade como o IME.

Meus sinceros agradecimentos à professora Cristina Gomes Fernandes e ao professor José Coelho de Pina, que não foi citado na capa apesar de ter sido a peça fundamental para esta dissertação. Agradeço pela amizade, forma dedicada e paciente com que me orientaram durante todo o período do mestrado.

Agradeço aos meus pais, Massagi e Eliana, por todo amor, carinho e dedicação durante todos os dias de minha vida, e aos meus irmãos, Eduardo e Beatriz, pelas brincadeiras, risadas e companhia.

Aos meus tios Newton e Toshiko e aos meus avós Tatsuma e Yolanda por todo amor e apoio.

Ao Renato Carmo pelo apoio durante a fase inicial deste trabalho e ao Shigueo e Wagner Dias pela amizade e ajuda durante os períodos de graduação e de pós.

Aos amigos Cebola, William, Henrique e Masaishi pelas aulas de tênis e pelo prazer de suas companhias.

Aos colegas Rodrigo, Eduardo, Mauricio, Romão, Ruth, Hamada, Vaz, Herbert, Leirê, Agnês, Tadao, Said, Fábio, Autoexec, Roy, Leston, Claus, Gruber e a todos aqueles que não foram citados, mas que estão presentes em meu coração.

Um obrigado especial aos professores Zé Augusto, Nami, Feofiloff, Carlinhos, Antônio Elias, Yoshiko, Siang e Mandel pela atenção e por todos os ensinamentos que adquiri.

À FAPESP pelo apoio financeiro e a todos os funcionários do IME.

Finalmente, um obrigado muitíssimo especial à minha namorada Ayumi pela paciência, amor e por todo carinho. 


\title{
Resumo
}

O problema da planaridade consiste em: dado um grafo $G$, decidir se $G$ é ou não é planar. A resposta deve vir acompanhada de uma justificativa. Se $G$ é planar, então uma justificativa é uma representação de um desenho de $G$ no plano sem cruzamento de arestas. Se $G$ não é planar, então uma justificativa é uma subdivisão do $K_{3,3}$ ou do $K_{5} \mathrm{em} G$.

Existem vários algoritmos lineares para testar planaridade. Dois deles são bem conhecidos: o algoritmo proposto por Auslander e Parter [1], posteriormente corrigido por Goldstein [9] (APG), e o algoritmo proposto por Lempel, Even e Cederbaum [12] (LEC).

Hopcroft e Tarjan [10] apresentaram em 1974 a primeira implementação linear do algoritmo de APG. Pouco depois, Booth e Lueker apresentaram uma implementação linear do algoritmo de LEC, introduzindo uma nova estrutura de dados chamada PQ-árvore. Recentemente, Shih e Hsu [15] e Boyer e Myrvold [3] publicaram duas implementações lineares do algoritmo de LEC que evitam o uso da PQ-árvore.

Este trabalho apresenta uma descrição do algoritmo de LEC e uma descrição da implementação de Shih e Hsu, bem como um estudo comparativo desta implementação com as implementações de Hopcroft e Tarjan, Booth e Lueker e de Boyer e Myrvold.

\begin{abstract}
The planarity testing problem consists of: given a graph $G$, decide whether $G$ is planar or not. The answer must be followed by a justification. If $G$ is planar, then a justification is a representation of a drawing of $G$ in the plane with no crossing edges. If $G$ is not planar, then a justification is a subdivision of $K_{3,3}$ or $K_{5}$ in $G$.

There are several linear-time algorithms to test planarity. Two of them are well known: the algorithm proposed by Auslander and Parter [1], later corrected by Goldstein [9] (APG), and the algorithm proposed by Lempel, Even e Cederbaum [12] (LEC).

In 1974, Hopcroft and Tarjan [10] presented the first linear-time implementation of APG's algorithm. Not long after that, Booth and Lueker presented a linear-time implementation of LEC's algorithm introducing a new data structure named PQ-tree. Recently, Shih and Hsu [15] and Boyer and Myrvold [3] proposed two linear-time implementations of LEC's algorithm that avoids using $\mathrm{PQ}$-tree.

This work presents a description of LEC's algorithm and a description of Shih and Hsu's implementation, as well as a comparative study involving this implementation, Hopcroft and Tarjan's, Booth and Lueker's and Boyer and Myrvold's implementation.
\end{abstract}




\section{Índice}

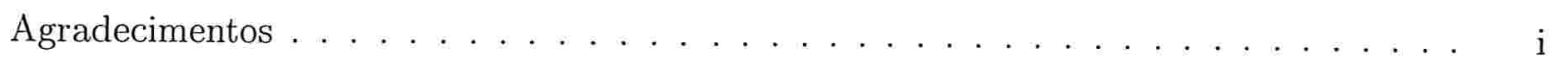

$\operatorname{Resumo\ldots \ldots \ldots \ldots \ldots \ldots \ldots \ldots \ldots \ldots \ldots \ldots \ldots \ldots \ldots \ldots \ldots }$

Lista de Figuras $\quad$ vii

$\begin{array}{ll}\text { Introdução } & 1\end{array}$

1 Preliminares $\quad 7$

1.1 Teoria dos grafos . . . . . . . . . . . . . . . 7

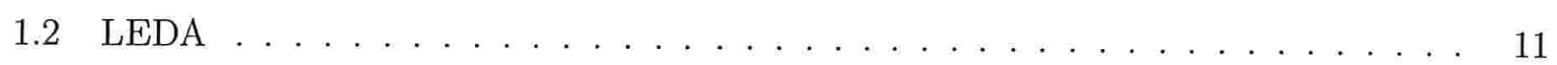

2 Planaridade $\quad 15$

2.1 Desenhos, faces, grafos planares e grafos planos . . . . . . . . . . . 15

2.2 Fórmula de Euler . . . . . . . . . . . . . . . . . . . . 16

2.3 Grafos de Kuratowski $\ldots \ldots \ldots \ldots \ldots \ldots$

2.4 Descrições combinatórias e ciclos faciais . . . . . . . . . . . . . . . . 19

2.5 Critério combinatório de planaridade . . . . . . . . . . . . . . 21

2.6 Formulação dos problemas . . . . . . . . . . . . . . . . . . . 23

3 Algoritmo de Lempel, Even e Cederbaum 25

3.1 Molduras, $X Y$-caminhos e $X Y$-obstruções . . . . . . . . . . . . . . 26

3.2 Lec-ordenação . . . . . . . . . . . . . . . . . . . . . . . . . . 32

3.3 Descrição do algoritmo . . . . . . . . . . . . . . . . . . . . 34 
3.4 Invariantes . . . . . . . . . . . . . . . . . . . 35

3.5 Correção . . . . . . . . . . . . . . . . . . . . 36

3.6 Teorema de Kuratowski . . . . . . . . . . . . . . . . . . . . 42

3.7 Eficiência . . . . . . . . . . . . . . . . . . . . . . . . . . 42

4 Implementação de Shih e Hsu 49

4.1 Ordem de processamento dos vértices . . . . . . . . . . . . . . . . . . . 49

4.2 Estrutura de dados para a moldura . . . . . . . . . . . . . . . . . 49

4.3 Algoritmo de $\mathrm{SH} \ldots \ldots \ldots \ldots \ldots \ldots \ldots \ldots$

4.4 Valores de $b$ para cada nó . . . . . . . . . . . . . . . . . . . 54

4.5 Percurso numa PC-árvore alterada . . . . . . . . . . . . . . . 57

4.6 Determinação dos terminais . . . . . . . . . . . . . . . . . 59

4.7 Busca por uma $X Y$-obstrução . . . . . . . . . . . . . . . . 63

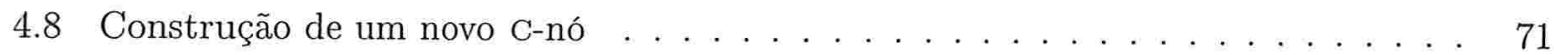

4.9 Correção . . . . . . . . . . . . . . . . . . . . 76

4.10 Eficiência . . . . . . . . . . . . . . . . . . . 81

5 Construção de um desenho plano $\quad 85$

5.1 Alterações na PC-árvore e no corpo do algoritmo . . . . . . . . . . . . . . . 85

5.2 Construção da DCP de um novo C-nó . . . . . . . . . . . . . . . 87

5.3 Correção . . . . . . . . . . . . . . . . . . . . . . 98

5.4 Eficiência . . . . . . . . . . . . . . . . . . . . 100

6 Obstruções 101

6.1 Informações adicionais para o cálculo da lista de $\operatorname{arcos} \ldots \ldots$. . . . . . . . . 101

6.2 Obstrução para o teste do contador de um C-nó . . . . . . . . . . . . . . . 101

6.3 Obstrução para cnode_of_possible_k33_obstruction . . . . . . . . . . 105

6.4 Cálculo de uma subdivisão de $K_{5} \ldots \ldots \ldots \ldots$. . . . . . . . . 107

6.5 Subdivisão de $K_{3,3}$ devido ao grau de $w 1 \ldots \ldots \ldots \ldots \ldots \ldots \ldots \ldots$

6.6 Subdivisão de $K_{3,3}$ com parte do ciclo externo de um C-nó . . . . . . . . . . . . 113

6.7 Subdivisão de $K_{3,3}$, subindo em $T_{0} \ldots \ldots \ldots \ldots \ldots \ldots \ldots \ldots \ldots$

6.8 Correção . . . . . . . . . . . . . . . . . . . . . . . 117 
7 Resultados experimentais

7.1 Ambiente experimental . . . . . . . . . . . . . . . . . . . . 120

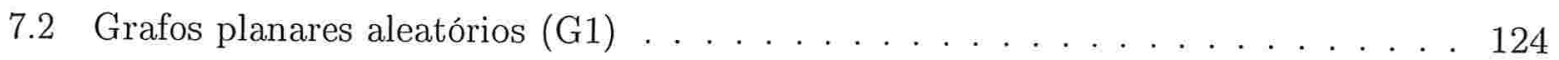

7.3 Grafos não-planares do tipo $(\mathrm{G} 2) \ldots \ldots \ldots \ldots \ldots \ldots \ldots$

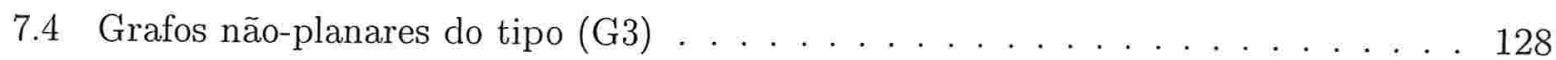

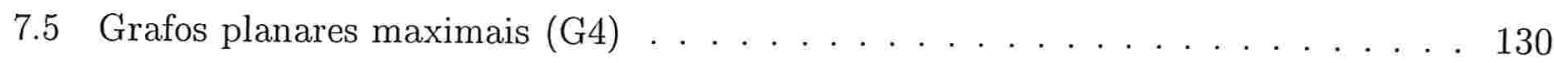

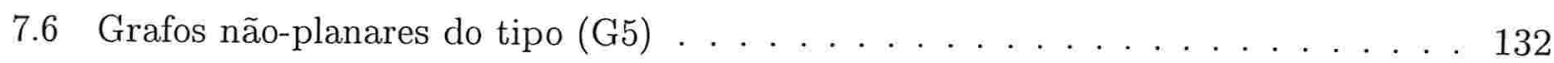

8 Comentários finais $\quad 135$

9 Índice 137

9.1 Blocos do código . . . . . . . . . . . . . . . . . . 137

$\begin{array}{lr}\text { Referências Bibliográficas } & 139\end{array}$

$\begin{array}{ll}\text { Índice Remissivo } & 141\end{array}$ 



\section{Lista de Figuras}

$1 \quad$ Desenhos . . . . . . . . . . . . . . . . . . . . . . . . 1

2 Grafos não-planares . . . . . . . . . . . . . . . . . . . . . . . 1

3 Grafos de Kuratowski . . . . . . . . . . . . . . . . . . . 2

4 Alguns algoritmos de planaridade . . . . . . . . . . . . . . 4

1.1 Ilustração de grafos . . . . . . . . . . . . . . . . . . . 8

1.2 Uma bp-árvore $T$ de um grafo e uma bp-ordenação induzida por $T$. . . . . . . . 10

1.3 Representação de um grafo por listas de adjacências . . . . . . . . . . . . . . . . . 11

1.4 Declarações de grafo, vértices e arcos no LEDA . . . . . . . . . . . . . . . 12

1.5 Construção de um grafo no LEDA . . . . . . . . . . . . . . . . . 12

1.6 Associando informações a vértices e arcos no LEDA . . . . . . . . . . . . . . 13

1.7 Exemplo de um programa em LEDA . . . . . . . . . . . . . . . . . . 14

2.1 Desenhos . . . . . . . . . . . . . . . . . . . . . 15

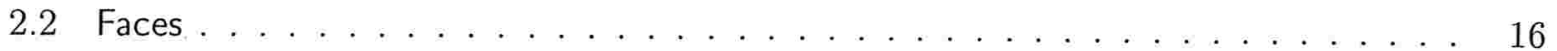

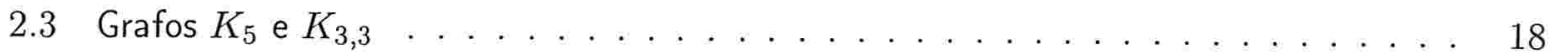

2.4 Subdivisão de uma aresta . . . . . . . . . . . . . . . . . . . . . . . . 19

2.5 Ordem cíclica dos arcos induzida por um desenho . . . . . . . . . . . . . . 20

2.6 Descrições combinatórias . . . . . . . . . . . . . . . . . . . 20

2.7 Ciclos faciais . . . . . . . . . . . . . . . . . . . . . . 21

2.8 Todos os arcos com uma mesma ponta num mesmo ciclo facial . . . . . . . . . . . 22

2.9 Dois ciclos faciais se fundem após a remoção de uma aresta . . . . . . . . . . . . . 23 
2.10 Critério combinatório para verificação de uma descrição plana

3.1 Moldura . . . . . . . . . . . . . . . . . . . . . . 26

3.2 Subgrafos induzidos de grafos não-planares que não possuem moldura . . . . . . . 27

3.3 Subgrafo induzido de um grafo planar que não possui moldura . . . . . . . . . . . 27

3.4 Caminho perimetral . . . . . . . . . . . . . . . . . 28

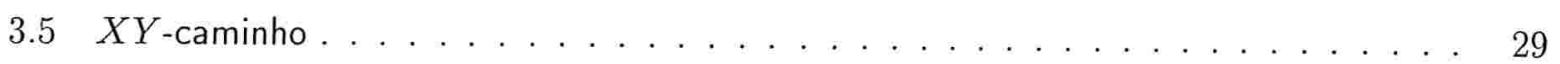

3.6 Uma lec-ordenação de um grafo . . . . . . . . . . . . . . . . . . . 33

3.7 Um grafo que não possui uma lec-ordenação . . . . . . . . . . . . . . . . 33

3.8 Construção de uma nova moldura . . . . . . . . . . . . . . . . 37

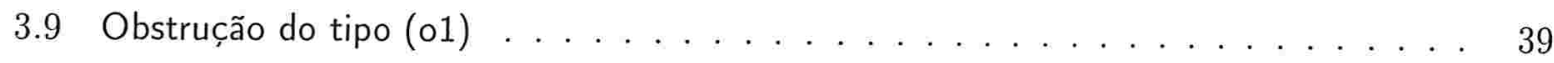

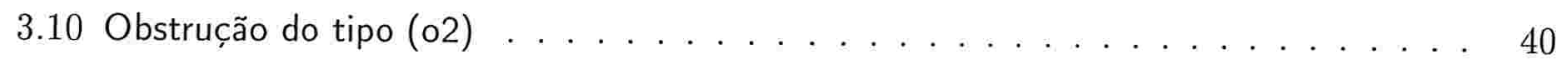

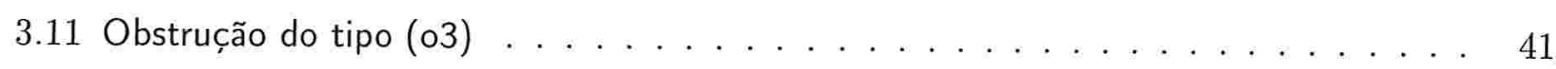

3.12 Cálculo de uma lec-ordenação. . . . . . . . . . . . . . . . . . . . . . 44

3.13 Árvore de blocos . . . . . . . . . . . . . . . . . . . . 45

4.1 PC-árvore . . . . . . . . . . . . . . . . . . . . . . 51

4.2 Declaração de uma PC-árvore alterada . . . . . . . . . . . . . . . . 53

4.3 Estrutura de dados de uma PC-árvore alterada . . . . . . . . . . . . . . . . . 54

4.4 Função is_planar . . . . . . . . . . . . . . . . . . . . 55

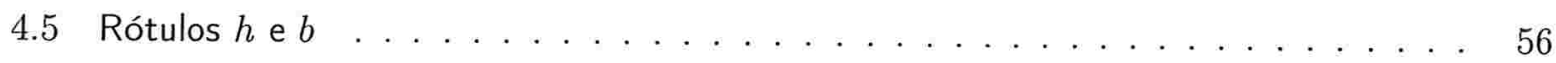

4.6 Função find_active_cnode . . . . . . . . . . . . . . . . . . 57

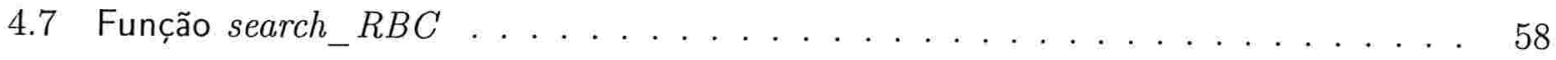

4.8 Cálculo de um C-nó ativo . . . . . . . . . . . . . . . . . . . . 59

4.9 Marca visitedpara aumentar eficiência . . . . . . . . . . . . . . 61

4.10 Função find_terminals . . . . . . . . . . . . . . . . . . . . 62

4.11 Função find_obstruction - parte $1 \ldots \ldots \ldots \ldots \ldots$

4.12 Função find_obstruction - parte $2 \ldots \ldots \ldots \ldots \ldots$. . . . . . . . . . 64

4.13 Função test_obstruction_from_terminal . . . . . . . . . . . . . . 65

4.14 Teste de obstrução para um terminal . . . . . . . . . . . . . . . 66

4.15 Teste de obstrução para dois terminais . . . . . . . . . . . . . . . . 67 
4.16 Função test_cnode_counter . . . . . . . . . . . . . . . . . . 68

4.17 Função find_node_with_label_b_greater_than_dfs_w . . . . . . . . 69

4.18 Função update_label_b . . . . . . . . . . . . . . . . . . . . 70

4.19 Função set_info_for_new_cnode . . . . . . . . . . . . . . . . 71

4.20 Função calculate_new_RBC . . . . . . . . . . . . . . . . . 73

4.21 Função calc_new_RBC_from_terminal_node . . . . . . . . . . . . . . 74

4.22 Função $a d d_{-}$old_cnode $R B C_{-} t o_{-} n e w_{-} R B C-$ parte $1 \ldots \ldots \ldots . \ldots 74$

4.23 Função add_old_cnode_ $R B C_{-} t_{-}$new $_{-} R B C$ - parte $2 \ldots \ldots \ldots \ldots$

4.24 "Flip" de um C-nó . . . . . . . . . . . . . . . . . . . . . . . . . . . 75

4.25 Simulação de uma PC-árvore alterada - parte $1 \ldots \ldots$. . . . . . . . 77

4.26 Simulação de uma PC-árvore alterada - parte $2 \ldots \ldots \ldots$. . . . . . . . 78

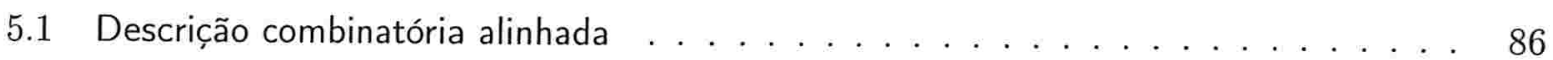

5.2 Função calculate_partial_embedding-parte $1 \ldots \ldots \ldots$

5.3 Função calculate_partial_embedding-parte $2 \ldots \ldots \ldots$. . . . . . 87

5.4 Ordenação das arestas de retorno em grupos . . . . . . . . . . . . . . . . 88

5.5 Função mark_path_in_t . . . . . . . . . . . . . . . . 89

5.6 Função group_back_edges_by_repr-parte $1 \ldots \ldots \ldots \ldots$

5.7 Função group_back_edges_by_repr-parte $2 \ldots \ldots \ldots \ldots \ldots$

5.8 Função embed_upward_t . . . . . . . . . . . . . . . . . . . 91

5.9 Função embed_back_edges - parte $1 \ldots \ldots \ldots$. . . . . . . . . . 92

5.10 Função embed_back_edges - parte 2 . . . . . . . . . . . . . . . . . 93

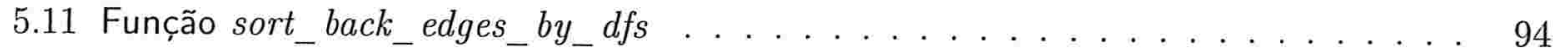

5.12 Inclusão das arestas em $T_{v}^{*}$ ao DCP de um novo C-nó $\ldots \ldots \ldots$. . . . . . 95

5.13 Função add_old_cnode_to_embedding . . . . . . . . . . . . . . 96

5.14 DCP's da simulação da PC-árvore alterada . . . . . . . . . . . . . . . . 97

5.15 Descrição combinatória plana para o grafo na figura 4.1 (a) . . . . . . . . . . 98

5.16 Arcos em torno de um P-nó $v$ não-terminal . . . . . . . . . . . . . . . . . 99

5.17 Arcos em torno de um P-nó $v$ terminal . . . . . . . . . . . . . . . . . . 99

5.18 Arcos devido a um C-nó ativo $v \ldots \ldots \ldots \ldots \ldots$. . . . . . . . . . . . . . . . . . . 
6.1 Função obstruction_edges_cnode_counter . . . . . . . . . . . . . 102

6.2 Obstrução pelo contador de um C-nó . . . . . . . . . . . . . . . . . 103

6.3 Função find_edge . . . . . . . . . . . . . . . . . . . . . . . . . 104

6.4 Função list_edges_upward_To . . . . . . . . . . . . . . . . . . . . 104

6.5 Função extract_boundary_cycle . . . . . . . . . . . . . . . 105

6.6 Função obstruction_edges_possible_obstr_confirmed . . . . . . . . . . 106

6.7 Obstrução para cnode_of_possible_k33_obstruction . . . . . . . . . . . 107

6.8 Obstrução do tipo (o1) para o caso de dois terminais . . . . . . . . . . . . . 108

6.9 Função obstruction_edges_K5 . . . . . . . . . . . . . . . . . . . . . 109

6.10 Cálculo de uma subdivisão de $K_{5} \ldots \ldots \ldots \ldots \ldots \ldots$. . . . . . . . 109

6.11 Função obstr_edges_pnode. . . . . . . . . . . . . . . . . . . . . . . . 109

6.12 Função obstr_edges_terminal . . . . . . . . . . . . . . . . . . . 110

6.13 Função obstruction_edges_countmin_ 1 . . . . . . . . . . . . . . . 111

6.14 Subdivisão de $K_{3,3}$ devido ao grau do menor ancestral de $w$ na obstrução . . . . . . 111

6.15 Função sort_by_label_b . . . . . . . . . . . . . . . . . . . . 112

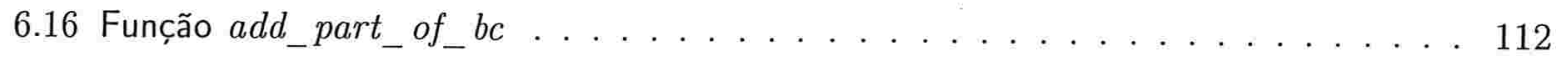

6.17 Função obstruction_edges_countmin_2_3 . . . . . . . . . . . . . 113

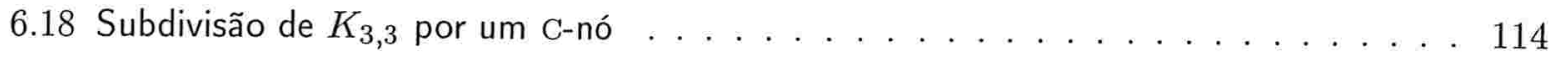

6.19 Função obstruction_edges_T0 . . . . . . . . . . . . . . . . . 115

6.20 Subdivisão de $K_{3,3}$ subindo em $T_{0} \ldots \ldots \ldots \ldots \ldots \ldots \ldots$

$6.21 v$ pode ser descendente de $m$ em $T_{0} \ldots \ldots \ldots \ldots \ldots \ldots \ldots$

6.22 Função active_cnode_of . . . . . . . . . . . . . . . . . 117

7.1 Função used_time. . . . . . . . . . . . . . . . . . . . . . . . . 120

7.2 Função maximal_planar_map . . . . . . . . . . . . . . . . 121

7.3 Função random_planar_map . . . . . . . . . . . . . . . 122

7.4 Saída da função print_statistics do LEDA . . . . . . . . . . . . . . . . . . 122

7.5 Tabela de comparação de tempos entre as implementações . . . . . . . . . . . . . 123

7.6 Função random_planar_graph . . . . . . . . . . . . . . . . . . . . 124

7.7 Espaço consumido para grafos (G1) . . . . . . . . . . . . . . . . . . 124

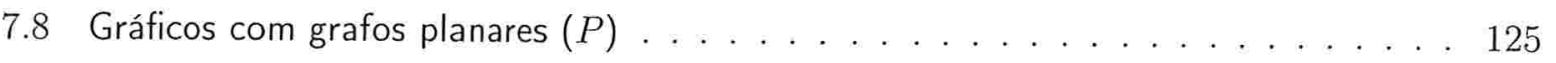


7.9 Trecho de código para gerar grafos não-planares do tipo (G2) . . . . . . . . . 126

7.10 Espaço consumido para grafos $(\mathrm{G} 2) \ldots \ldots \ldots \ldots \ldots \ldots \ldots$

7.11 Gráficos com grafos não-planares $\left(P+K_{3,3}\right) \ldots \ldots \ldots \ldots \ldots$. . . . . . . 127

7.12 Trecho de código para gerar grafos não-planares do tipo (G3) . . . . . . . . . 128

7.13 Espaço consumido para grafos $(G 3) \ldots \ldots \ldots \ldots \ldots$

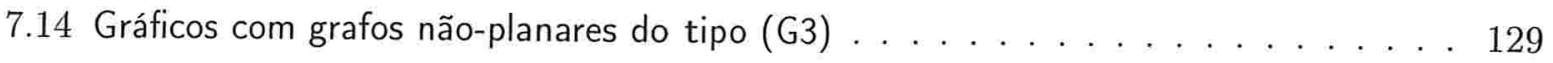

7.15 Rotina maximal_planar_graph . . . . . . . . . . . . . . . . 130

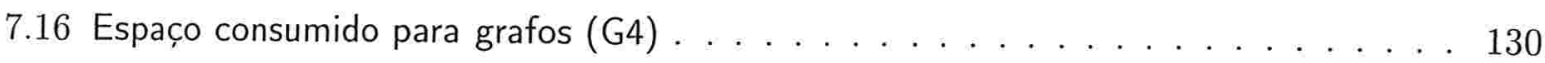

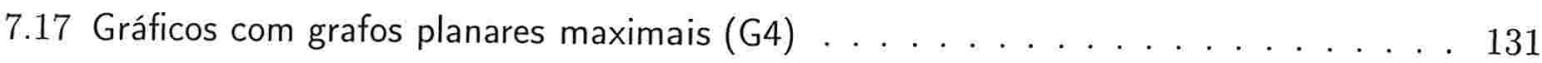

7.18 Trecho de código (do LEDA) para gerar grafos não-planares do tipo (G5) . . . . . 132

7.19 Espaço consumido para grafos $(G 5) \ldots \ldots \ldots \ldots \ldots$. . . . . . . . . . . . . . . . . . . .

7.20 Gráficos com grafos não-planares do tipo $(G 5) \ldots \ldots \ldots \ldots$ 


\section{Introdução}

Um desenho de um grafo no plano tal que suas arestas se intersectam somente nos seus pontos extremos, caso exista, é chamado de desenho plano do grafo e um grafo para o qual existe um desenho plano é dito planar (veja figura 1).

Existem situações práticas onde é necessário determinar se um dado grafo possui um desenho plano. Por exemplo, em projetos de circuitos VLSI, existe o interesse em obter-se um desenho desse tipo que representa um circuito elétrico.

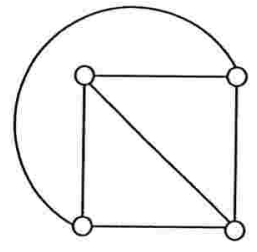

(a)

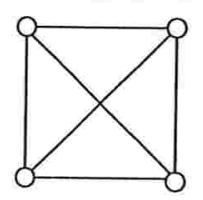

(b)

Figura 1: (a) Um desenho plano de um grafo planar. (b) Um desenho que não é plano do mesmo grafo.

Os grafos da figura 2 não são planares.

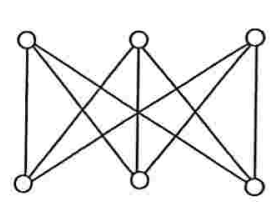

(a)

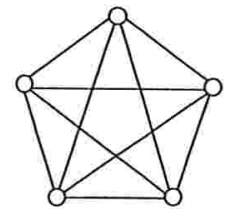

(b)

Figura 2: Exemplos de grafos não-planares. (a) $K_{3,3}$. (b) $K_{5}$. 
Uma caracterização muito simples de grafos planares foi dada por Kuratowski [11]. Ele demonstrou que os grafos $K_{3,3}$ e $K_{5}$ são, basicamente, os únicos grafos não-planares: os grafos da figura 3 são chamados de grafos de Kuratowski e um grafo é planar se e somente se ele não contém um subgrafo de Kuratowski.

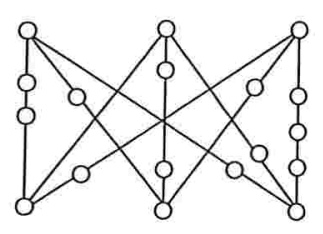

(a)

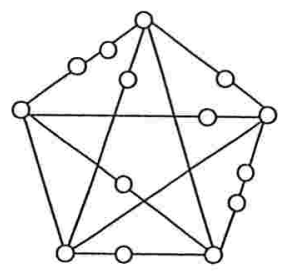

(b)

Figura 3: Grafos de Kuratowski. (a) Cada aresta do $K_{3,3}$ é substituída por um caminho de comprimento pelo menos um. (b) $\mathrm{O}$ mesmo para o $K_{5}$.

Apesar de elegante, a caracterização de Kuratowski não fornece um algoritmo prático para o teste de planaridade. Ademais, não está claro como obter um desenho plano de um grafo planar através dessa caracterização.

Uma possível estratégia para decidir se um grafo $G$ é planar é construir incrementalmente um desenho plano de $G$. Se for possível completar a construção de um desenho plano de $G$, então $G$ é planar, caso contrário, idealmente, um subgrafo de Kuratowski é encontrado em $G$, justificando a não planaridade de $G$. O primeiro algoritmo incremental para teste de planaridade foi proposto por Auslander e Parter [1]. Dado um grafo $G$, primeiramente um circuito de $G$ é encontrado. O grafo resultante da remoção desse circuito de $G$ é composto de 'partes' conexas. O algoritmo é chamado recursivamente para obter um desenho plano de cada uma dessas partes junto com o circuito. Depois, esses desenhos planos são combinados, se possível, para obter um desenho plano de $G$. Se não for possível combinar esses desenhos planos, um subgrafo de Kuratowski é encontrado em G. O algoritmo de Auslander e Parter continha um erro que foi corrigido por Goldstein [9], resultando no algoritmo de Auslander, Parter e Goldstein (APG). A complexidade de tempo desse algoritmo é cúbica.

Lempel, Even e Cederbaum [12] (LEC) apresentaram uma maneira alternativa de se construir um desenho plano incrementalmente. Eles começam com o desenho de um único vértice e adicionam ao desenho todas as arestas incidentes a este vértice. Depois adicionam um novo vértice incidente a uma das arestas já inseridas e todas as arestas incidentes a este novo vértice. O processo continua dessa maneira. Cada iteração do algoritmo começa com um desenho plano de parte do grafo e consiste em adicionar um novo vértice e todas as arestas incidentes a este vértice ao desenho plano corrente. O processo continua até que todo o grafo tenha sido desenhado no plano ou tenha-se detectado que o grafo não é planar. Este tipo de algoritmo incremental é 
chamado de vertex addition algorithm. Tarjan [17] apresentou uma implementação do algoritmo de LEC que consome tempo quadrático.

Em 1974, Hopcroft e Tarjan [10] apresentaram o primeiro algoritmo linear para decidir se um dado grafo é planar. O algoritmo de Hopcroft e Tarjan é uma implementação envolvente do algoritmo de APG.

Hopcroft e Tarjan não deram muitos detalhes de como o seu algoritmo de teste de planaridade pode ser estendido para construir de fato um desenho plano do grafo dado. Uma descrição completa da fase de construção de um desenho plano por esse algoritmo foi feita por Mehlhorn e Mutzel [13] e a respectiva implementação por Mutzel [14]. Até hoje não se conhece uma implementação do algoritmo de Hopcroft e Tarjan que, em tempo linear, devolve um certificado de não planaridade, ou seja, um subgrafo de Kuratowski de um grafo não-planar.

Em 1976, Booth e Lueker [2] apresentaram uma implementação do algoritmo de LEC que consome tempo linear. Para isto, Booth e Lueker empregaram uma estrutura de dados chamada de PQ-árvore. Recentemente, Shih e Hsu [15] e Boyer e Myrvold [3] descreveram implementações similares para o algoritmo de LEC. Ambas gastam tempo linear e evitam o uso de PQ-árvores.

Os dois algoritmos lineares para teste de planaridade mais discutidos na literatura são os algoritmos de APG e o de LEC. Canfield e Williamson [5] fazem uma comparação entre esses dois algoritmos argumentando que os dois, geralmente vistos como métodos bem diferentes, têm na realidade muitas semelhanças.

Esta dissertação apresenta uma descrição do algoritmo de LEC e uma descrição da implementação proposta por Shih e Hsu [15]. Neste trabalho, a implementação de Shih e Hsu foi desenvolvida sobre a plataforma LEDA [7]. Além disso, é feito um estudo comparativo entre esta implementação, as duas implementações presentes no LEDA (uma implementação de Hopcroft e Tarjan [10] e uma implementação de Booth e Lueker [2]) e a implementação de Boyer e Myrvold [3].

A figura 4 mostra um resumo do consumo de tempo de algumas implementações dos principais algoritmos de planaridade existentes até hoje.

\section{Organização da dissertação}

O capítulo 1 contém a maior parte da notação, conceitos e definições usados ao longo desta dissertação. Em seguida, no capítulo 2, são apresentados alguns conceitos e resultados sobre planaridade que são fundamentais para a descrição do algoritmo de LEC [12], presente no capítulo 3, e para a descrição da implementação desse algoritmo proposta por Shih e Hsu [15], nos capítulos seguintes. 


\begin{tabular}{|l|rr|}
\hline \hline Algoritmo & Implementação & Complexidade \\
\hline \hline Auslander, Parter e Goldstein (APG) & original [1,9] & cúbico \\
& Hopcroft e Tarjan [10] & linear \\
\hline Lempel, Even e Cederbaum (LEC) & original [12] & quadrático \\
& Booth e Lueker [2] & linear \\
& Boyer e Myrvold [3] & linear \\
& Shih e Hsu [15] & linear \\
\hline
\end{tabular}

Figura 4: Tabela dos principais algoritmos de planaridade e algumas de suas implementações.

No capítulo 7 é apresentada uma análise experimental entre as implementações de Booth e Lueker (BL) [2], Hopcroft e Tarjan (HT) [10], Boyer e Myrvold (BM) [3] e de Shih e Hsu (SH) [15]. Finalmente, no capítulo 8, encontra-se um resumo da nossa experiência durante o desenvolvimento deste trabalho.

\section{Código fonte da implementação de Shih e Hsu}

A implementação de Shih e Hsu foi desenvolvida pelos autores dessa dissertação sobre a plataforma LEDA, versão 4.4, e testada num computador pessoal com sistema GNU/Linux.

Para compilar o código fonte da implementação, é necessário possuir o LEDA instalado no seu sistema, além de um compilador $\mathrm{C}++$.

A versão mais recente do LEDA para avaliação pode ser obtida pela internet, no endereço http://www.algorithmic-solutions.com/ e o pacote compactado do código fonte das implementações de $\mathrm{SH}$ e de BM podem ser obtidos pela internet, no endereço http://www.ime.usp.br/ noma/sh/planarity.tar.gz.

Para descompactar o arquivo planarity.tar.gz no diretório meudir/, utilize o comando

meu_prompt> tar -zxvpf planarity.tar.gz -C meudir/

Após a descompactação, o diretório meudir/planarity/ é criado. O código fonte da implementação de BM foi gentilmente cedido por John M. Boyer, cuja página pessoal se encontra no endereço http://pacificcoast.net/ lightning/jboyer.htm.

Para compilar os programas planarity_time e sh_planar, basta executar meu_prompt> make 
planarity_time. Este programa imprime, na saída padrão, uma tabela de comparação de tempos, em formato AATEX, entre as implementações de BL, HT, BM e de SH.

\section{Código fonte da dissertação}

Para compilar a dissertação, é necessário possuir o LEDA, o LATEX e o noweb devidamente instalados no seu sistema. ( $\mathrm{O}$ noweb é uma ferramenta para programação literária (literate programming).)

Uma versão do noweb pode ser obtida pela internet, no endereço http://www . eecs. harvard. edu/ nr/noweb.

O código fonte da dissertação pode ser obtido pela internet, no endereço http://www.ime.usp.br/ noma/sh/dissertacao.tar.gz.

Para descompactar o arquivo dissertação.tar.gz no diretório meudir/, utilize o comando

meu_prompt> tar -zxvpf dissertacao.tar.gz -C meudir/

Após a descompactação, o diretório meudir/dissertacao/ é criado.

Para compilar a dissertação, execute

meu_prompt $>$./mkdissertacao

O arquivo dissertacao.ps.gz é criado. 


\section{Preliminares}

Neste capítulo é apresentada a maior parte da notação e definições usadas ao longo desta dissertação.

\subsection{Teoria dos grafos}

Esta seção introduz os conceitos de grafos, conexidade, planaridade e outros elementos básicos da teoria dos grafos.

\section{Grafos orientados, subgrafos orientados e grafos}

Um grafo orientado é um objeto da forma $(V, A)$, onde $V$ é um conjunto finito de elementos, chamados vértices, e $A$ é um conjunto de pares ordenados de elementos distintos de $V$, chamados arcos. Se $V=\emptyset$ então o grafo é vazio.

Se $G$ é um grafo orientado, o conjunto dos vértices de $G$ é denotado por $V_{G}$ e o conjunto dos arcos de $G$ por $A_{G}$.

Um arco $(u, v)$ é denotado por $u v$. Diz-se que $v u$ é o reverso de $u v$. Se $u v$ é um arco, então $u$ e $v$ são as pontas inicial e final de $u v$, respectivamente. Diz-se também que $u$ e $v$ são vizinhos e que $u v$ sai de $u$ e entra em $v$. Se vu é um arco, então diz-se que $u v$ tem reverso.

Diz-se que $H$ é um subgrafo orientado de $G$ se $H$ é um grafo orientado tal que $V_{H} \subseteq V_{G}$ e $A_{H} \subseteq A_{G}$. Se $H$ contém todos os arcos de $G$ com ambas as pontas em $V_{H}$ então $H$ é um subgrafo induzido de $G$. Neste caso, denota-se $H$ por $G\left[V_{H}\right]$ e diz-se que $H$ é o subgrafo de $G$ induzido por $V_{H}$.

Se $V \subseteq V_{G}$ então $G-V$ é o subgrafo orientado de $G$ resultante da remoção de todos os vértices de $V$ e de todos os arcos com pelo menos uma ponta em $V$. Ou seja, $G-V$ é o grafo $G\left[V_{G} \backslash V\right]$.

$V_{G}$

$G\left[V_{H}\right]$

$G-V$ 
Seja $H$ um subgrafo induzido de $G$ e $w$ um vértice de $G$. Denota-se por $H+w$ o grafo $G\left[V_{H} \cup\{w\}\right]$. Similarmente, denota-se por $H-w$ o grafo $G\left[V_{H} \backslash\{w\}\right]$.

Um grafo (simétrico) é um grafo orientado em que todo arco tem reverso. Num grafo, um conjunto $\{u v, v u\}$ é chamado de aresta. Abusando da notação, uma aresta $\{u v, v u\}$ é denotada simplesmente por $u v$ ou $v u$. Dada uma aresta $u v$, diz-se que $u v$ é incidente a $u$ e a $v$ e que $u$ e $v$ são as pontas de $u v$. Além disso, $u$ e $v$ são ditos adjacentes.

Se $u$ é um vértice, então o grau de $u$ é o número total de arestas incidentes a $u$.

$E_{G}$

Se $G$ é um grafo, $E_{G}$ é o conjunto das arestas de $G$ e os números $\left|V_{G}\right|$ e $\left|E_{G}\right|$ são denotados, respectivamente, por $n$ e $m$. O tamanho de $G$ é o número $n+2 m$.

Um grafo pode ser naturalmente representado através de um diagrama como o da figura 1.1(a), onde os vértices são pequenos círculos, os arcos são setas ligando os dois círculos que correspondem as suas pontas. Geralmente esse diagrama é simplificado para aquele representado na figura 1.1(b), onde cada arco, junto com seu reverso, é substituído por uma linha simples.

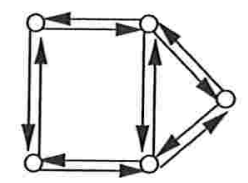

(a)

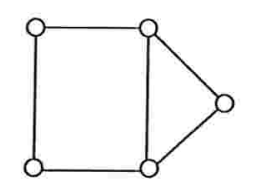

(b)

Figura 1.1: (a) Diagrama de um grafo. (b) Versão simplificada do diagrama.

\section{Passeios, ciclos, caminhos e circuitos}

Um passeio num grafo orientado é uma seqüência

$$
\left\langle v_{0}, a_{1}, v_{1}, \ldots, v_{k-1}, a_{k}, v_{k}\right\rangle
$$

onde $v_{0}, v_{1}, \ldots, v_{k}$ são vértices e, para cada $i, a_{i}$ é o arco $v_{i-1} v_{i}$. Diz-se que o passeio é entre $v_{0}$ e $v_{k}$, ou que começa em $v_{0}$ e termina em $v_{k}$ e que $v_{0}$ e $v_{k}$ são as pontas do passeio. Tal passeio é muitas vezes denotado por $\left\langle v_{0}, v_{1}, \ldots, v_{k-1}, v_{k}\right\rangle$. O comprimento de um passeio $P$ é o número $|P|=k$ e os vértices $v_{1}, \ldots, v_{k-1}$ são os vértices internos do passeio. Um ciclo num grafo $G$ é um subgrafo orientado de $G$ formado pelos vértices e arcos de um passeio que começa e termina num mesmo vértice. Um ciclo determinado pelo passeio $\left\langle v_{0}, v_{1}, \ldots, v_{k-1}, v_{0}\right\rangle$ é denotado por $\left\langle v_{0}, v_{1}, \ldots, v_{k-1}, v_{0}\right\rangle$. Um caminho é um passeio sem repetição de vértices. Um circuito é um passeio $\left\langle v_{0}, v_{1}, \ldots, v_{k-1}, v_{k}\right\rangle$ de comprimento pelo menos três em que $v_{0}, v_{1}, \ldots, v_{k-1}$ são vértices distintos e $v_{k}=v_{0}$. 
As vezes confundimos um grafo ou um passeio com seu conjunto de arestas. Seja $P$ um caminho tal que $E_{P}$ é vazio. Então $P$ é dito trivial.

\section{Conexidade, blocos e grafos bipartidos}

Um grafo é conexo se, para todo par de vértices $u$ e $v$ do grafo, existe um caminho entre $u$ e $v$. Um componente de um grafo é um subgrafo conexo maximal. O grafo vazio é conexo.

Um vértice de corte de um grafo $G$ é um vértice $v$ tal que $G-v$ tem mais componentes que $G$. Um grafo é biconexo se é conexo e não possui vértices de corte. Um subgrafo biconexo maximal é chamado de bloco.

Um grafo $G$ é bipartido se $V_{G}$ pode ser particionado em dois conjuntos $R$ e $S$ tais que, para cada aresta $u v$ de $G$, se $u$ é um vértice de $R$ então $v$ está em $S$, e se $u$ é um vértice de $S$ então $v$ está em $R$.

\section{Árvores, árvores enraizadas e busca em profundidade}

Uma árvore é um grafo conexo sem circuitos. Numa árvore, existe um único caminho entre de qualquer vértice a qualquer outro.

Uma árvore com um vértice especial $r$ é dita enraizada. O vértice $r$ é chamado de raiz.

Seja $v$ um vértice de uma árvore $T$ com raiz $r$. Qualquer vértice $u$ no caminho de $r$ a $v$ em $T$ é chamado de ancestral de $v$ em $T$. Um ancestral $u$ de $v$ é próprio se $u \neq v$. Se $u$ é um ancestral de $v$, então $v$ é um descendente de $u$. Um descendente $v$ de $u$ é próprio se $v \neq u$. Se $v \neq r$ e $u v$ é a última aresta do caminho de $r$ a $v$, então $u$ é o pai de $v$ e $v$ é um filho de $u$ em $T$. Dois vértices com o mesmo pai são chamados de irmãos.

Uma árvore geradora de um grafo conexo $G$ é um subgrafo de $G$ que é uma árvore e contém todos os vértices de $G$.

A subárvore de $T$ com raiz $u$ cujos vértices são todos os descendentes de $u$ em $T$ é denotada por $T_{u}$.

Uma floresta é um grafo tal que cada um de seus componentes é uma árvore.

Uma bp-árvore (ou uma árvore de busca em profundidade) de um grafo conexo $G$ é uma árvore geradora enraizada de $G$ tal que, para cada aresta $u v$ em $G$, ou $u$ é descendente de $v$ na árvore ou $v$ é descendente de $u$ (figura 1.2(b)).

Seja $T$ uma bp-árvore de $G$. Uma ordenação $v_{1}, v_{2}, \ldots, v_{n}$ dos vértices de $G$ tal que se $v_{i}$ é ancestral de $v_{j}$ em $T$ então $i>j$ 
é dita uma bp-ordenação de $G$ (em relação a $T$ ). Veja a figura 1.2. O bp-número de um

$\operatorname{bp}(u)$ vértice $u$ (em relação a uma bp-ordenação), denotado por $\operatorname{bp}(u)$, é o índice $i$ tal que $u=v_{i}$. Note que se $r$ é a raiz de $T$ então $b p(r)=n$.

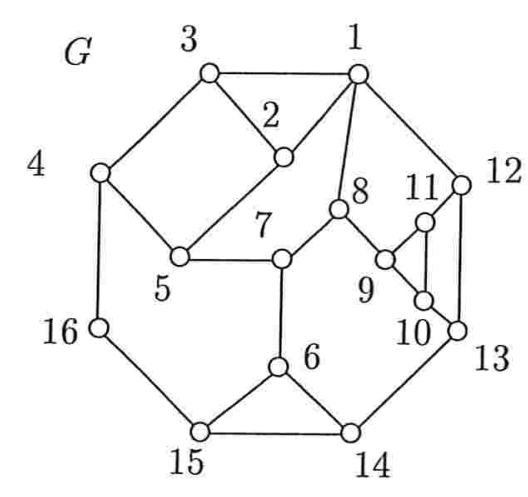

(a)

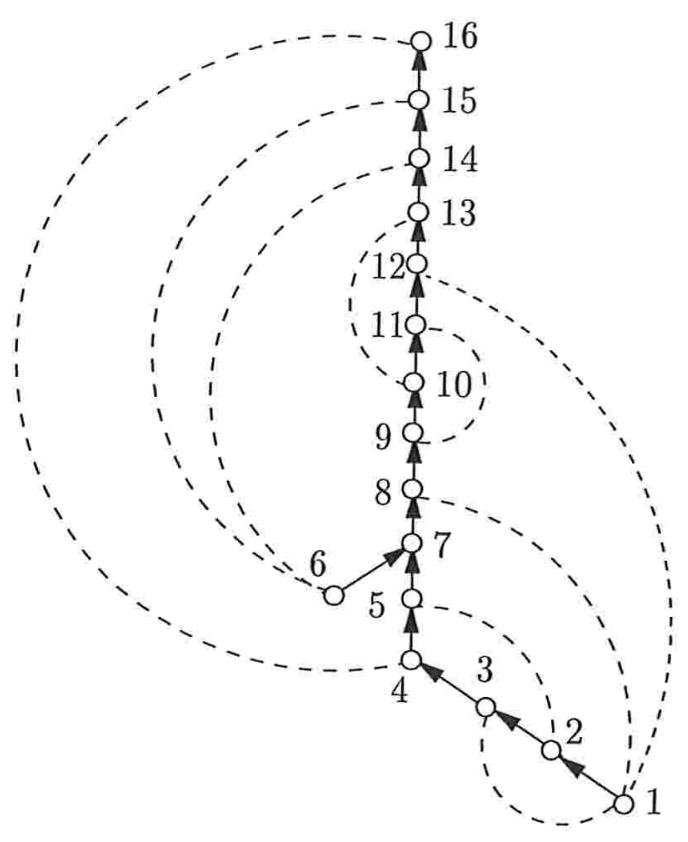

(b)

Figura 1.2: (a) Um grafo $G$ com vértices numerados de acordo com uma bp-ordenação. (b) Uma bp-árvore $T$ de $G$, indicada por linhas sólidas, enraizada no vértice 16. As setas apontam para o pai de cada nó em $T$.

\section{Representação de grafos em um computador}

Um grafo pode ser representado em um computador pelas suas listas de adjacências. A $\operatorname{Adj}(u)$ lista de adjacência de um vértice $u$, denotada por $\operatorname{Adj}(u)$, consiste de uma lista que contém todos os arcos com ponta inicial u (figura 1.3(a)). O espaço consumido por esta representação é $O(n+m)$.

Note que as listas de adjacências de um grafo podem ser representadas por uma lista única de arcos (figura 1.3(b)) e pode-se converter de uma representação para a outra em tempo $O(n+m)$. 


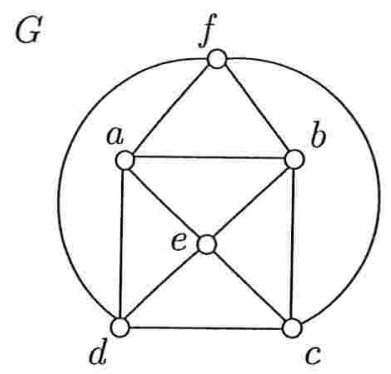

Listas de adjacências:

$$
\begin{aligned}
& \operatorname{Adj}(a)=\langle a f, a b, a e, a d\rangle \\
& \operatorname{Adj}(b)=\langle b c, b e, b a, b f\rangle \\
& \operatorname{Adj}(c)=\langle c d, c e, c b, c f\rangle \\
& \operatorname{Adj}(d)=\langle d f, d a, d e, d c\rangle \\
& \operatorname{Adj}(e)=\langle e a, e b, e c, e d\rangle \\
& \operatorname{Adj}(f)=\langle f c, f b, f a, f d\rangle
\end{aligned}
$$

(a)

Representação por uma lista:

$$
\langle a f, b c, b e, a b, b a, f c, b f, c d, c e, c b, c f, a e, d f, d a, d e, d c, e a, e b, e c, a d, e d, f b, f a, f d\rangle
$$

(b)

Figura 1.3: (a) Um grafo e uma representação deste por listas de adjacências. (b) Representação das listas de adjacências por uma única lista de pares de vértices.

\subsection{LEDA}

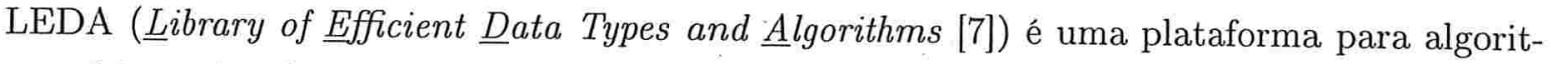
mos combinatórios desenvolvida, inicialmente, por Näher e Mehlhorn. Ela foi utilizada para a implementação de Shih e Hsu descrita neste trabalho.

Abaixo encontra-se uma breve apresentação de como representar grafos orientados, vértices e arcos no LEDA e os comandos usados para criar e percorrer vértices e os arcos de um dado grafo. São apresentadas também algumas estruturas de dados do LEDA que são utilizadas neste trabalho.

\section{Grafos orientados, vértices e arcos}

Um grafo orientado é representado no LEDA pelo tipo graph, um vértice pelo tipo node e um arco pelo tipo edge (figura 1.4).

As funções source(uv) e target(uv) devolvem, respectivamente, as pontas inicial e final de um arco uv. Ambas gastam tempo constante.

O número de vértices e de arcos de um grafo são obtidos, respectivamente, pelas funções number_of_nodes e number_of_edges. Ambas consomem tempo constante. 


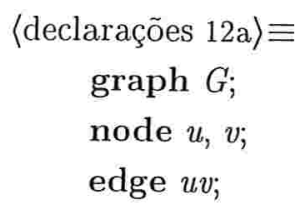

Figura 1.4: A variável $G$ representa um grafo orientado vazio. As variáveis $u, v$ são apontadores para vértices e $u v$ é um apontador para um arco. Inicialmente, $u, v$ e $u v$ estão com valores indefinidos.

\section{Manipulação de grafos}

Para adicionar um vértice e um arco a um grafo $G$, usa-se, respectivamente, as funções new_node e new_edge (figura 1.5). Para remover um vértice e um arco de $G$, usa-se, respectivamente, as funções del_node e del_edge. Estas quatro funções consomem tempo constante.

$$
\begin{aligned}
& u=G . n e w_{-} \text {node }() ; \\
& v=G . n e w_{-} \text {node }() ; \\
& u v=G . n e w_{-} \text {edge }(u, v) ; \\
& \text { G.new_edge(G.new_node }(), u) ;
\end{aligned}
$$

Figura 1.5: Construção de um grafo no LEDA.

Para percorrer todos os vértices e arcos de $G$, usa-se, respectivamente, as macros forall_nodes e forall_edges (figura 1.6). Elas gastam tempo proporcional a $\left|V_{G}\right|$ e a $\left|E_{G}\right|$, respectivamente.

\section{Listas e vetores}

Para representar uma lista de elementos de um mesmo tipo, usa-se o tipo list e para representar um vetor de elementos, usa-se o tipo array. Por exemplo, list<edges $>$ é usado para declarar uma lista de arestas.

Para inserir um elemento no início de uma lista, usa-se a função push e para inserir um elemento no fim de uma lista, usa-se a função append. Um apontador para um elemento de uma lista é do tipo item_list. Dado um apontador para um elemento de uma lista, usa-se a função del para remover o elemento indicado pelo apontador.

Para percorrer todos os elementos de uma lista, usa-se a macro forall. 


\section{bmlist}

O tipo bmlist, proposto por Boyer e Myrvold [3], consiste de uma lista que pode ser invertida em tempo constante. Nela, cada célula possui dois apontadores (campos ptr1 e ptr2) e, para passar para o próximo elemento na lista, olha-se os dois apontadores e escolhe-se o apontador que não leva ao elemento anteriormente percorrido. Assim, para inverter essa lista, basta trocar as células que são os extremos da lista.

Para percorrer todos os elementos de uma bmlist, foi criado a macro myforall.

\section{Associando informações a vértices e arcos de um grafo}

Para atribuir informações a todos os vértices e arcos de um grafo $G$, usa-se vetores indexados por vértices e arcos, respectivamente (figura 1.6). Os vetores indexados por vértices são os tipos node_array e node_map. A diferença entre estes é que o primeiro é usado para grafos estáticos e o segundo para grafos dinâmicos. Analogamente, os vetores indexados por arcos são os tipos edge_array e edge_map.

Os tipos map $<$ node, $\ldots>$ e map $<$ edge,$\ldots>$ podem ser usados nos lugares de node_map e edge_map, respectivamente. O tipo map é útil quando deseja-se associar informações a um pequeno número de vértices ou arcos do grafo.

O tempo gasto para acessar cada elemento de um vetor node_array ou edge_array é constante, enquanto que o tempo gasto esperado para os tipos map, node_map e edge_map é constante.

node_array $<$ int $>$ nodenumber $(G)$;

edge_array $<$ int $>$ edgenumber $(G)$;

int $i=0$;

node $w$;

forall_nodes $(w, G)$ nodenumber $[w]=+i$;

$i=0$;

edge $e$;

forall_edges $(e, G)$ edgenumber $[e]=+i$;

Figura 1.6: Enumeração dos vértices e arcos do grafo construído na figura 1.5.

\section{Estrutura de um programa}

Um exemplo de um programa em LEDA é ilustrado na figura 1.7. 
Dados dois elementos $a$ e $b$, a função leda_swap $(a, b)$ troca o conteúdo de $a$ pelo conteúdo de $b$ e vice-versa.

Dados dois arcos $a$ e $b$, a função set_reversal $(a, b)$ é usada para guardar a informação de que $a$ é o reverso de $b$ e vice-versa. Dado um arco, o seu reverso (caso exista tal informação) é devolvido pelas funções reverse e reversal.

14

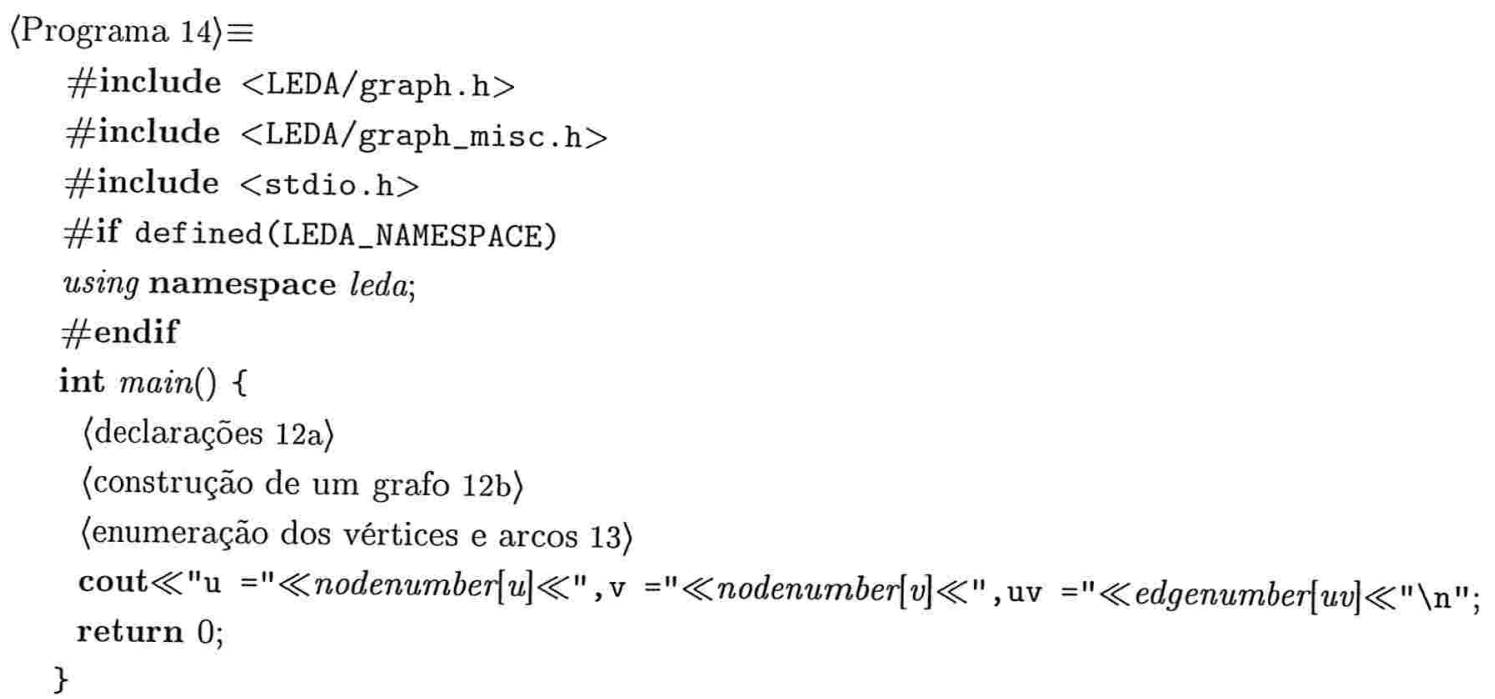

Figura 1.7: Exemplo de um programa em LEDA. Como saída, o programa imprime $u=1, v=$ $2, u v=1$. 


\section{Planaridade}

Neste capítulo, são apresentados alguns ingredientes básicos que envolvem planaridade. Conceitos topológicos de planaridade tais como curvas e desenhos são tratados de maneira superficial. A intenção é trocar, o mais rapidamente possível, esses elementos topológicos por objetos combinatórios que, do ponto de vista algorítmico deste trabalho, são de fundamental importância. Assim, este capítulo pode ser encarado como uma (rápida) transição da intuição topológica para a combinatória de planaridade e estruturas de dados.

\subsection{Desenhos, faces, grafos planares e grafos planos}

Um desenho de um grafo $G$ é uma função $I$ que associa a cada vértice $v$ de $G$ um ponto distinto $I(v)$ no plano e associa a cada aresta $u v$ uma curva $I(u v)$, sem auto-intersecção, de $I(u)$ a $I(v)$ no plano, i.e., $I(u v)$ é uma função injetora contínua do intervalo $[0,1]$ no plano tal que $I(u v)(0)=I(u)$ e $I(u v)(1)=I(v)$. Na figura 2.1 são apresentados três desenhos diferentes de um mesmo grafo.

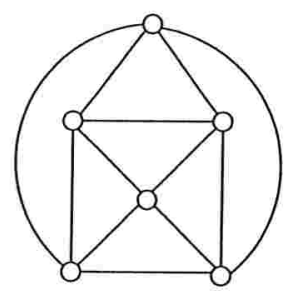

(a)

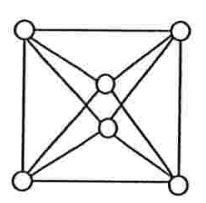

(b)

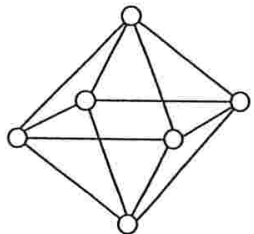

(c)

Figura 2.1: Três desenhos diferentes de um mesmo grafo. 
Um desenho de um grafo decompõe o plano em regiões formadas por pontos que podem ser ligados por curvas que não intersectam a imagem do desenho. Essas regiões são as faces do desenho. A região ilimitada é chamada de face externa (figura 2.2).

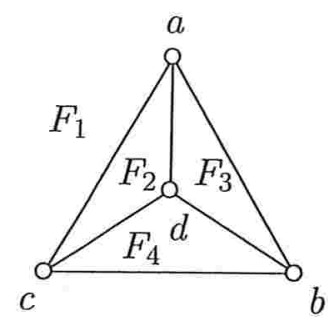

(a)

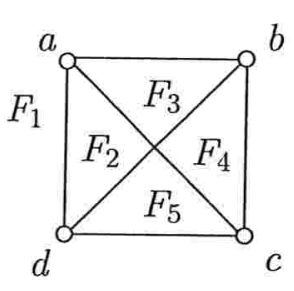

(b)

Figura 2.2: Dois desenhos diferentes de um mesmo grafo e suas respectivas faces. Em (a) e (b), $F_{1}$ é a face externa.

Um desenho $I$ de um grafo $G$ é plano se

(a) a imagem das arestas não contêm imagens dos vértices no seu interior, ou seja, $I(u v)(x) \neq$ $I(w)$ para toda aresta $u v$, vértice $w$ e número real $x$ no intervalo $(0,1)$;

(b) as imagens de arestas distintas não se intersectam no seu interior, ou seja, $I(u v)(x) \neq$ $I(s t)(y)$ para quaisquer arestas distintas $u v$ e $s t$ e números reais $x$ e $y$ no intervalo $(0,1)$.

Na figura 2.1 somente (a) é um desenho plano. Um grafo $G$ é dito planar se ele admite um desenho plano. O grafo vazio é planar. Um grafo planar junto com um de seus desenhos planos é chamado de grafo plano. Neste trabalho, quando dizemos que $G$ é um grafo plano, estamos implicitamente considerando um desenho plano de $G$. Assim, por exemplo, faz sentido falar-se no número de faces de um grafo plano.

\subsection{Fórmula de Euler}

Uma fórmula simples relacionando o número de vértices, de arestas e de faces de um grafo plano conexo foi dada por Euler.

Teorema 2.1 (fórmula de Euler): Se $G$ é um grafo plano conexo com $n$ vértices, $m$ arestas e $f$ faces, então

$$
n-m+f=2 \text {. }
$$

Demonstração: Fixamos $n$ e aplicamos indução em $m$. Se $G$ é uma árvore, então $m=n-1$, $f=1$ e a fórmula é válida. Agora suponha que $G$ não é uma árvore. Então $G$ possui uma aresta $e$ 
tal que $G^{\prime}:=G-e$ é um grafo conexo e, evidentemente, plano. Sejam $n^{\prime}, m^{\prime}$ e $f^{\prime}$ o número de vértices, arestas e faces de $G^{\prime}$. É claro que $n^{\prime}=n, m^{\prime}=m-1$ e $f^{\prime}=f-1$. Finalmente, por indução, temos que

$$
2=n^{\prime}-m^{\prime}+f^{\prime}=n-(m-1)+(f-1)=n-m+f .
$$

Do teorema acima seguem alguns corolários. Cada um deles fornece uma delimitação superior para o número de arestas de um grafo planar.

Corolário 2.2: Se G é um grafo planar com $m$ arestas, $n$ vértices, $n \geq 3$, então

$$
m \leq 3 n-6
$$

Demonstração: Podemos supor que se $u$ e $v$ são vértices de $G$ tais que $u v$ não é aresta de $G$, então $G+u v$ é não-planar, já que esta operação faz crescer o lado esquerdo da desigualdade acima e mantém constante o lado direito. É claro que $G$ é conexo. Considere um desenho plano de $G$ e seja $f$ o número de faces desse desenho. Como $n \geq 3$, então cada face do desenho possui exatamente três arestas em sua fronteira. Logo, $3 f=2 m$. Pela fórmula de Euler tem-se que

$$
2=n-m+f=n-m+(2 / 3) m=n-(1 / 3) m,
$$

donde

$$
m=3 n-6 .
$$

O menor comprimento de um circuito num grafo é a sua cintura. Se o grafo não possui circuito algum, então diz-se que a sua cintura é "infinita". O corolário abaixo pode ser visto como uma certa generalização do corolário 2.2 .

Corolário 2.3: Se $G$ é um grafo com $m$ arestas, $n$ vértices, e cintura (finita) $k, k \geq 3$, então

$$
m \leq(k /(k-2))(n-2) \text {. }
$$

Demonstração: Podemos supor que se $u$ e $v$ são vértices de $G$ tais que $u v$ não é aresta de $G$, então $G+u v$ é não-planar ou tem cintura menor que $k$. É claro que $G$ é conexo. Considere um desenho plano de $G$ e seja $f$ o número de faces desse desenho. Como $G$ tem cintura $k$, então cada face do desenho possui pelo menos $k$ arestas em sua fronteira. Logo, $k f \leq 2 m$. Pela fórmula de Euler tem-se que

$$
2=n-m+f \leq n-m+(2 / k) m=n-((k-2) / k) m,
$$


donde

$$
m \leq(k /(k-2))(n-2) \text {. }
$$

\subsection{Grafos de Kuratowski}

Os corolários no final da seção anterior podem ser usados para verificar que os grafos da figura 2.3 são não-planares. Os grafos das figuras $2.3(\mathrm{a})$ e (b) são denotadas por $K_{5}$ e $K_{3,3}$, respectivamente.

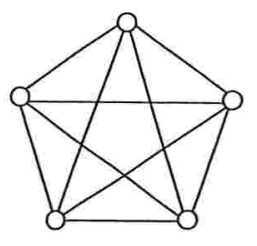

(a)

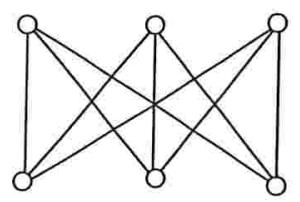

(b)

Figura 2.3: (a) $K_{5}$. (b) $K_{3,3}$.

Corolário 2.4: $K_{5}$ é não-planar.

Demonstração: Suponha por contradição que $K_{5}$ é planar. Então, pelo corolário 2.2, tem-se que $m=10 \leq 3 \times 5-6=9$, uma contradição. Portanto $K_{5}$ não pode ser planar.

Corolário 2.5: $K_{3,3}$ é não-planar.

Demonstração: Suponha por contradição que $K_{3,3}$ é planar. Então, pelo corolário 2.3, como $K_{3,3}$ tem cintura 4, tem-se que $m=9 \leq 2 \times 6-4=8$, uma contradição. Portanto $K_{3,3}$ não pode ser planar.

Subdividir uma aresta $u v$ de um grafo $G$ significa substituir $u v$ por um caminho de comprimento dois com pontas $u$ e $v$ (figura 2.4). Uma subdivisão de um grafo $G$ é um grafo que pode ser obtido a partir de $G$ por uma seqüência finita de subdivisões de arestas. Um grafo de Kuratowski é qualquer subdivisão do grafo $K_{5}$ ou do grafo $K_{3,3}$.

O lema a seguir será utilizado, no capítulo 3 , para obter uma caracterização de planaridade, conhecida por Teorema de Kuratowski. A essência desse teorema diz que a recíproca do lema abaixo é verdadeira. 


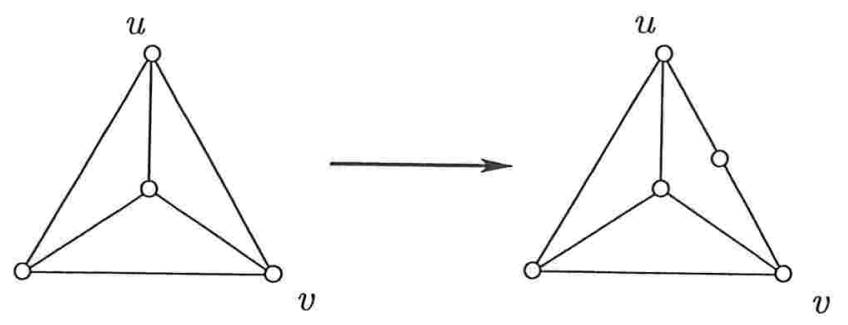

Figura 2.4: Subdivisão da aresta $u v$.

Lema 2.6: Se G é um grafo que possui um subgrafo de Kuratowski, então G é nãoplanar.

Demonstração: É claro que se $G$ possui um subgrafo que não é planar, então $G$ não é planar. Ademais, toda subdivisão de um grafo que não é planar também não é planar. Portanto o lema segue dos corolários 2.4 e 2.5 .

\subsection{Descrições combinatórias e ciclos faciais}

Convém lembrar que neste trabalho um grafo (simétrico) é um grafo orientado tal que $u v$ é um arco do grafo se e somente se o seu reverso vu é um arco do grafo. Em um grafo uma aresta $u v$ é um conjunto de arcos $\{u v, v u\}$. Assim, dependendo do contexto, algumas vezes é conveniente nos referirmos a aresta $u v$ e em outras oportunidades ao arco $u v$.

Um desenho de um grafo induz ${ }^{1}$ uma ordem cíclica nos arcos com ponta inicial em cada vértice (figura 2.5). Essa ordem pode ser horária ou anti-horária. Usaremos sempre a ordem horária. Uma lista formada por todas essas ordens cíclicas de arcos é chamada de uma descrição combinatória do desenho. Mais precisamente, uma lista $D$ é uma descrição combinatória de um desenho se, para cada vértice $v, D(v)$ é a ordem cíclica de todos os arcos com ponta inicial em $v$, induzida pelo desenho. Cada ordem cíclica é representada por uma lista de arcos.

É evidente que uma descrição combinatória pode corresponder a uma infinidade de desenhos. Note que as listas de adjacências de um grafo formam uma descrição combinatória de um desenho do grafo. Compare as figuras 2.6(a) e 1.3 da página 11.

Seja $D$ uma descrição combinatória de um desenho de um grafo. Um ciclo facial de $D$ é um ciclo $\left\langle v_{0}, v_{1}, \ldots, v_{k-1}, v_{0}\right\rangle$ onde, para cada $i, v_{i} v_{i+1}$ é o sucessor cíclico de $v_{i} v_{i-1}$ em $D\left(v_{i}\right)$, considerando-se os índices módulo $k$ (figura 2.7). Os ciclos faciais $C_{1}, C_{2}, C_{3}$ e $C_{4}$ da figura 2.7(a) correspondem as faces $F_{1}, F_{2}, F_{3}$ e $F_{4}$ da figura 2.2 , respectivamente.

\footnotetext{
${ }^{1}$ Esta ordem é induzida numa "vizinhança suficientemente pequena" de um vértice.
} 


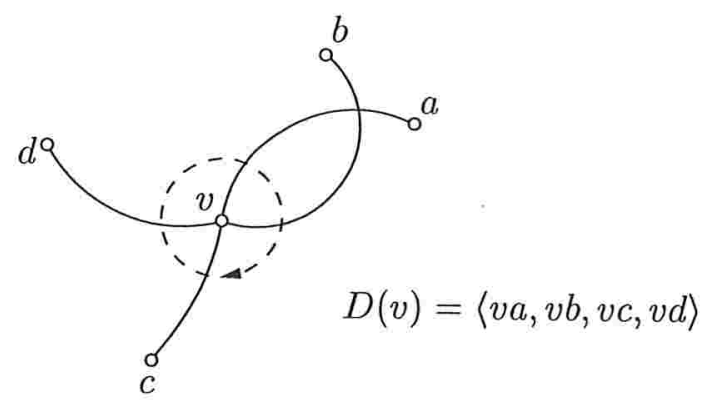

Figura 2.5: Ordem cíclica dos arcos com ponta inicial $v$ induzida por um desenho. A ordem cíclica $D(v)$ é representada pela lista $v a, v b, v c, v d$.

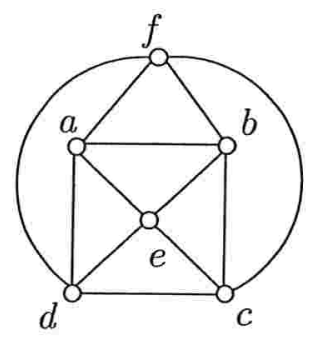

Descrição combinatória $D_{1}$ :

$D_{1}(a)=\langle a f, a b, a e, a d\rangle$

$D_{1}(b)=\langle b c, b e, b a, b f\rangle$

$D_{1}(c)=\langle c d, c e, c b, c f\rangle$

$D_{1}(d)=\langle d f, d a, d e, d c\rangle$

$D_{1}(e)=\langle e a, e b, e c, e d\rangle$

$D_{1}(f)=\langle f c, f b, f a, f d\rangle$

(a)

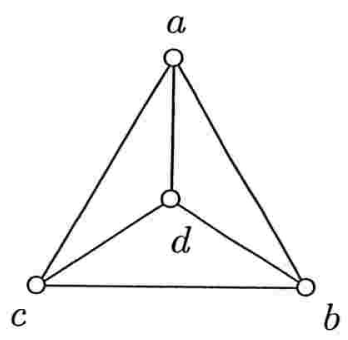

Descrição combinatória $D_{2}$ :

$D_{2}(a)=\langle a b, a d, a c\rangle$

$D_{2}(b)=\langle b c, b d, b a\rangle$

$D_{2}(c)=\langle c a, c d, c b\rangle$

$D_{2}(d)=\langle d a, d b, d c\rangle$

(b)

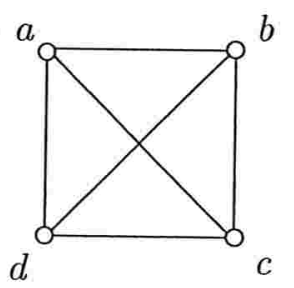

Descrição combinatória $D_{3}$ :

$D_{3}(a)=\langle a b, a c, a d\rangle$

$D_{3}(b)=\langle b c, b d, b a\rangle$

$D_{3}(c)=\langle c d, c a, c b\rangle$

$D_{3}(d)=\langle d a, d b, d c\rangle$

Figura 2.6: (a) Um desenho plano e uma descrição combinatória induzida pelo desenho. Em (b) e (c), são ilustrados dois desenhos de um mesmo grafo: um plano e um não-plano, respectivamente. 


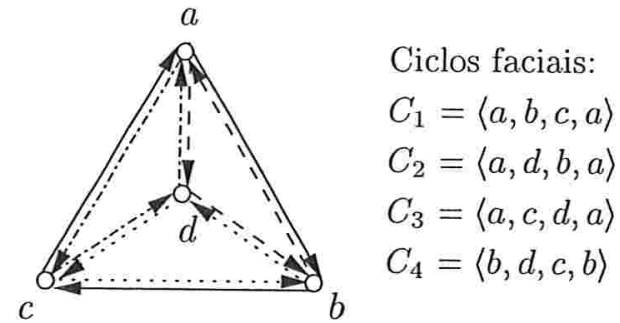

(a)

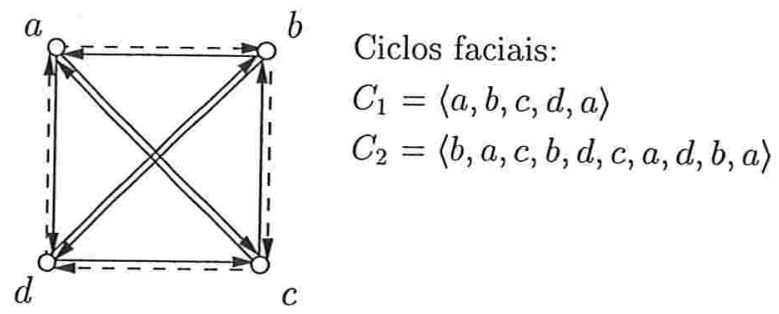

(b)

Figura 2.7: (a) Ciclos faciais determinados pela descrição combinatória da figura 2.6(b). (c) Ciclos faciais determinados pela descrição combinatória da figura 2.6(c).

Uma descrição combinatória de um desenho plano é dita plana. É claro que todo grafo planar possui uma descrição combinatória plana. Nas figuras 2.6(a) e (b) encontram-se dois desenhos planos e suas respectivas descrições combinatória planas.

No contexto deste trabalho, descrições combinatórias planas têm um papel fundamental. É fácil ver que que toda descrição combinatória de uma árvore é plana. Note ainda que se $D$ é uma descrição combinatória plana de um grafo com pelo menos uma aresta, então existe uma correspondência bijetora entre ciclos faciais de $D$ e faces do desenho: cada ciclo facial "corresponde" a fronteira de uma face ${ }^{2}$. Estas observações são usadas na próxima seção.

\subsection{Critério combinatório de planaridade}

Nesta seção é apresentado um critério combinatório para testar se uma dada descrição combinatória é ou não plana. Este critério é facilmente transformado num trecho de código usado para verificar a correção das respostas fornecida pelas implementações feitas neste trabalho.

Teorema 2.7: Seja $G$ um grafo conexo com pelo menos uma aresta. Uma descrição combinatória $D$ de um desenho de $G$ é plana se e somente se

$$
n-m+f_{D}=2
$$

onde $f_{D}$ é o número de ciclos faciais de $D$.

Demonstração: Suponha que $D$ é uma descrição combinatória de um desenho plano de $G$. Não é difícil ver que existe uma correspondência bijetora entre os ciclos faciais de $D$ e os ciclos na fronteira das faces do desenho. Logo, como $G$ tem pelo menos uma aresta e $f_{D}$ é igual ao número de faces do desenho, da fórmula de Euler, segue que (2.1) vale.

\footnotetext{
${ }^{2} \mathrm{E}$ óbvio que o nome "ciclo facial" se deva a esta correspondência.
} 
Reciprocamente, é demonstrado a seguir, por indução em $f_{D}$, que se $D$ é uma descrição combinatória que satisfaz (2.1), então ela $D$ é plana.

Como $G$ tem pelo menos uma aresta, então $f_{D} \geq 1$. Se $f_{D}=1$, então $n-m=1$ e portanto $G$ é uma árvore e $D$ é plana, já que toda descrição combinatória de uma árvore é plana.

Suponha agora que $f_{D} \geq 2$. Afirmamos que existem arcos $u v$ e $v u$ de $G$ que não pertencem a um mesmo ciclo facial. De fato, suponha que cada par $u v$ e $v u$ de $\operatorname{arcos}$ de $G$ pertencem a um mesmo ciclo facial e considere um vértice $v$ de $G$. Seja $D(v)=\left\langle v v_{0}, v v_{1}, \ldots, v v_{k}\right\rangle$ a ordem cíclica dos arcos com ponta inicial em $v$ (figura 2.8). Como $v v_{i+1}$ é o sucessor cíclico de $v v_{i}$ em $D(v)$ e $v_{i} v$ e $v v_{i}$ pertencem a um mesmo ciclo facial $(i=0,1, \ldots, k)$, então todos os arcos com uma ponta em $v$ pertencem a um mesmo ciclo facial . Como $G$ é conexo, então todos os arcos de $G$ pertencem a um mesmo ciclo facial. Portanto, $f_{D}=1$, uma contradição.

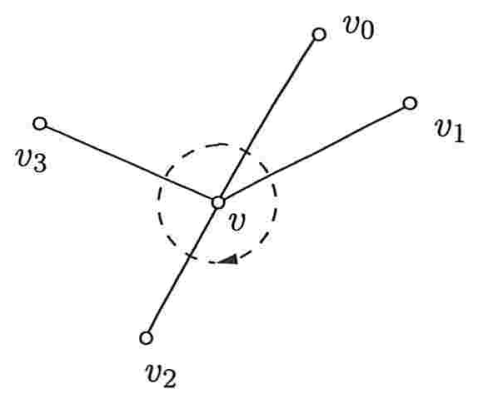

Figura 2.8: Todos os arcos com uma ponta em $v$ estão num mesmo ciclo facial.

Sejam $u v$ e $v u$ arcos de $G$ que não pertencem a um mesmo ciclo facial $D$. Sejam

$$
C_{u v}:=\left\langle v=w_{0}, w_{1}, \ldots, w_{t-1}=u, v=w_{0}\right\rangle \text { e } C_{v u}:=\left\langle u=z_{0}, z_{1}, \ldots, z_{l-1}=v, u=z_{0}\right\rangle
$$

os ciclos faciais de $D$ contendo $u v$ e $v u$, respectivamente (figura 2.9).

Devido a escolha de $u v$ e $v u$, tem-se que $G^{\prime}:=G-\{u v, v u\}$ é conexo e tem pelo menos uma aresta. Seja $D^{\prime}$ a descrição combinatória de $G^{\prime}$ induzida por $D$. Em $D^{\prime}$, os ciclos faciais $C_{u v}$ e $C_{v u}$ deram lugar ao ciclo facial

$$
C^{\prime}=\left\langle u=z_{0}=w_{t-1}, z_{1}, \ldots, z_{l-1}=v=w_{0}, w_{1}, \ldots, w_{t-1}=z_{0}=u\right\rangle
$$

e os demais ciclos faciais permanecem os mesmos. Logo, $f_{D^{\prime}}=f_{D}-1 \mathrm{e}$, por indução, $D^{\prime}$ é uma descrição combinatória de um desenho plano $I^{\prime}$ de $G^{\prime}$.

Como existe uma correspondência bijetora entre ciclos faciais de $D^{\prime}$ e faces de $I^{\prime}$, então $C^{\prime}$ corresponde a uma face $F^{\prime}$ de $I^{\prime}$. Pode-se estender o desenho plano $I^{\prime}$ de $G^{\prime}$ a um desenho plano $I$ de $G$ fazendo com que a imagem da aresta $u v$ por $I$ esteja contida em $F^{\prime}$. Tem-se que $D$ é descrição combinatória de $I$ e, portanto, é plana. 


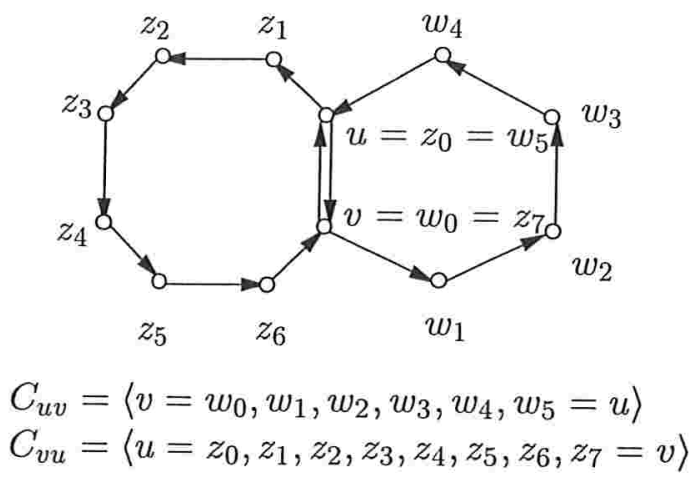

(a)

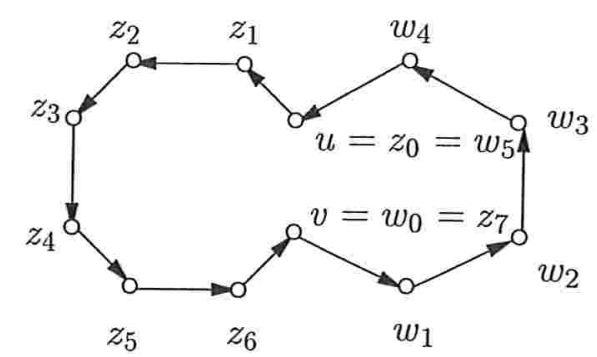

$\begin{aligned} C & =\left\langle u=z_{0}=w_{5}, z_{1}, z_{2}, z_{3}, z_{4}, z_{5}, z_{6}, v=\right. \\ & \left.=z_{7}=w_{0}, w_{1}, w_{2}, w_{3}, w_{4}, u=w_{5}=z_{0}\right\rangle\end{aligned}$

(b)

Figura 2.9: Dois ciclos faciais em (a) que se fundem em (b) após a remoção da aresta uv.

A função na figura 2.10 traduz o critério de planaridade para a linguagem da plataforma LEDA. O código supõe que o grafo dado é conexo e tem pelo menos uma aresta. Essa função devolve verdadeiro se a descrição combinatória é plana e falso caso contrário. (Uma descrição combinatória é dada pela representação do grafo no LEDA. O sucessor cíclico de um arco $e$, no ciclo facial que contém $e$, num grafo $G$ é acessado pela função $G$.face_cycle_succ(e).)

\subsection{Formulação dos problemas}

Há dois tipos de problemas relacionados a planaridade tratados neste trabalho. O mais simples é o de teste de planaridade.

Problema do teste de planaridade: Dado um grafo $G$, decidir se $G$ é planar.

O problema acima pede por uma simples resposta SIM ou NÃO:

- SIM, o grafo dado possui uma descrição combinatória plana;

- NÃO, o grafo dado não possui uma descrição combinatória plana.

Este problema, por ser mais simples, é o primeiro a ser tratado. Um algoritmo de teste de planaridade é apresentado no capítulo 3 e uma implementação é discutida no capítulo 4 .

Os capítulos 3 e 4 servirão de passos intermediários no caminho para entender e implementar um algoritmo para o problema da planaridade. 
$\langle$ Critério combinatório 24$\rangle \equiv$

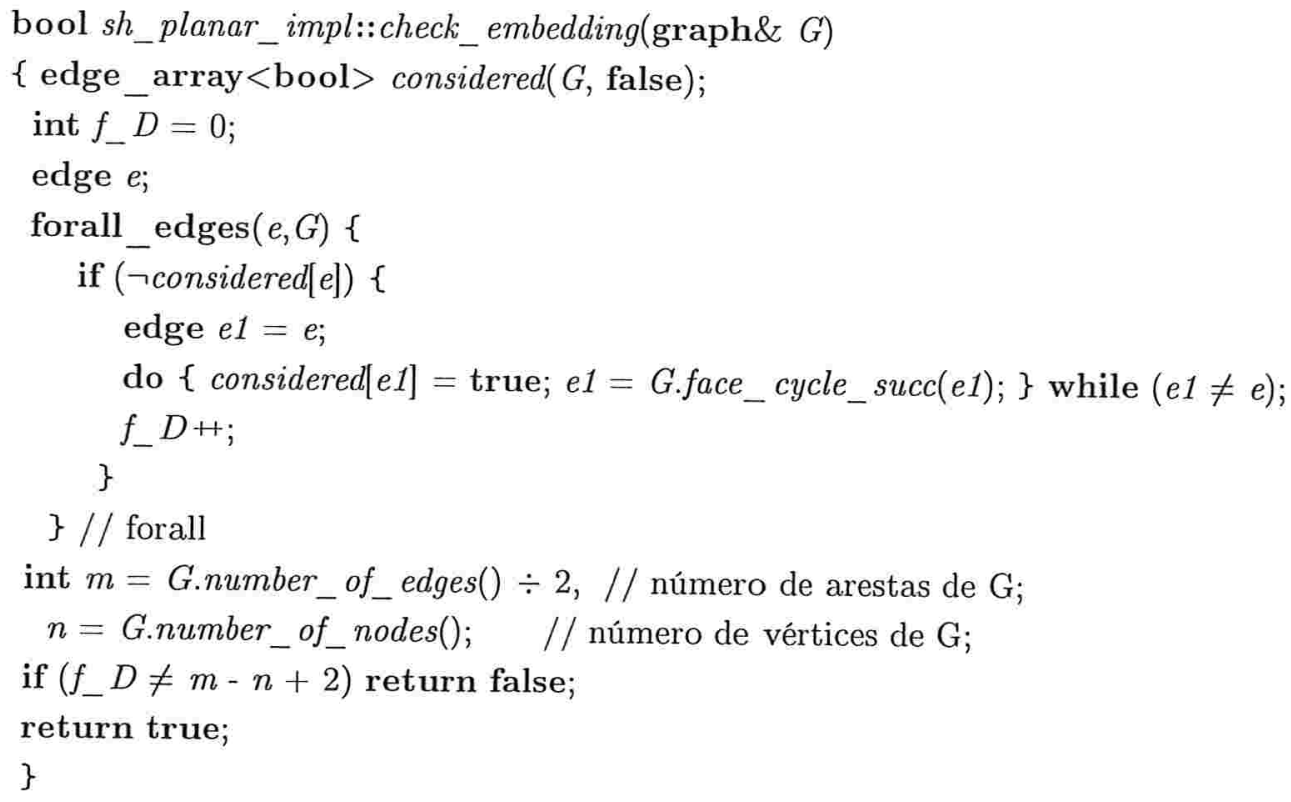

Figura 2.10: Critério combinatório para verificação de uma descrição combinatória plana. Recebe um grafo conexo $G$ com pelo menos uma aresta e uma descrição combinatória $D$ implícita nas listas de adjacência na representação de $G$. Devolve true se $D$ é plana e false caso contrário.

Problema da planaridade: Dado um grafo $G$, determinar uma descrição combinatória plana de $G$ ou um certificado de não-planaridade de $G$, ou seja, um subgrafo de Kuratowski em $G$.

A palavra certificado é usada de maneira vaga na descrição do problema acima, apesar de possuir um significado técnico bem preciso. Para os propósitos deste trabalho, basta saber que certificados típicos são subgrafos de Kuratowski, como mostra o capítulo 3.

Nos capítulos 5 e 6, o algoritmo de teste de planaridade (capítulo 3) e sua implementação (capítulo 4) são estendidos para resolver o problema da planaridade em tempo linear. 


\section{Algoritmo de Lempel, Even e Cederbaum}

Neste capítulo é descrito o algoritmo de Lempel, Even e Cederbaum [12] (LEC) que decide se um dado grafo é planar. O algoritmo de LEC examina cada vértice do grafo dado, um após o outro, em uma certa ordem chamada de st-ordenação (st-numbering). Em cada iteração, o algoritmo procura acrescentar o vértice sendo examinado ao desenho plano do subgrafo induzido pelos vértices já examinados.

A descrição original do algoritmo de LEC é feita através do conceito da chamada forma de bush (bush form). A forma de bush pode ser entendida como uma estrutura de dados que mantém toda a informação necessária para estender o desenho plano corrente a um desenho plano do grafo dado, se possível. Tarjan [17] apresentou uma implementação do algoritmo de LEC que consome tempo quadrático.

Em 1976, Booth e Lueker [2] empregaram a chamada PQ-árvore (PQ-tree) para representar uma forma de bush. A implementação resultante consome tempo linear.

Mais recentemente, Shih e Hsu [15] e Boyer e Myrvold [3] apresentaram novas implementações do algoritmo de LEC que consomem tempo linear. Shih e Hsu utilizaram uma estrutura de dados que, devido a sua semelhança com as PQ-árvores, foi batizada de PC-árvore. Boyer e Myrvold utilizaram uma estrutura de dados diferente das $\mathrm{PQ}$-árvores.

A descrição do algoritmo de LEC neste capítulo não é feita em termos de formas de bush. Em vez disso, a descrição utiliza o conceito de molduras (frames), que foi proposto por Thomas [18]. Além disso, este capítulo não trata de descrições combinatórias planas nem de imersões em geral. A versão do algoritmo de LEC descrita é de teste de planaridade. A intenção com isto é separar (o máximo possível) o algoritmo abstrato das (muito importantes) tecnicalidades da implementação, que serão tratadas nos próximos capítulos. Ao final deste capítulo é apresentada uma implementação não elaborada do algoritmo de LEC que consome tempo quadrático. 


\subsection{Molduras, $X Y$-caminhos e $X Y$-obstruções}

Thomas [18] descreve a implementação de Shih e Hsu [15] do algoritmo de LEC por meio do conceito de molduras, $X Y$-caminhos e $X Y$-obstruções. Uma descrição de cada um desses objetos e suas inter-relações vem a seguir.

\section{Molduras}

Seja $M$ um subgrafo de um grafo $H$. Diz-se que $M$ é uma moldura de $H$ se

(m1) existe um desenho plano de $H$ em que $M$ é o subgrafo induzido pelas arestas adjacentes à face externa.

É evidente que um grafo possui uma moldura se e somente se é planar.

Se $H$ é um subgrafo induzido de um grafo $G$ e $M$ é uma moldura de $H$, então $M$ é uma moldura de $H$ em $G$ se

(m2) todo vértice de $H$ vizinho de um vértice em $V_{G} \backslash V_{H}$ é um vértice de $M$.

A figura 3.1 mostra um subgrafo induzido e sua moldura.

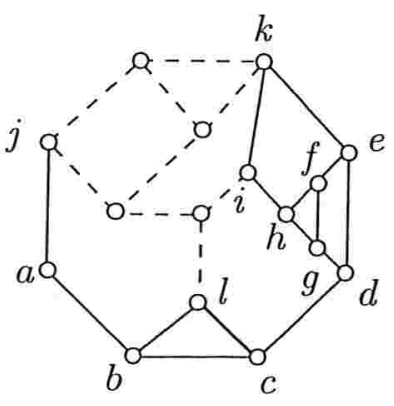

(a)

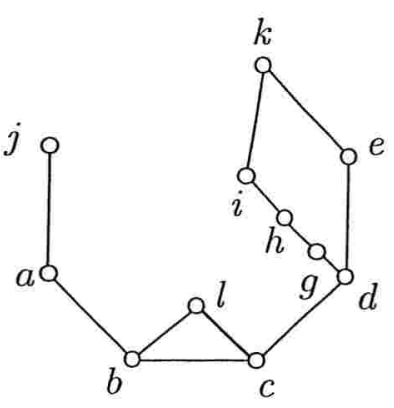

(b)

Figura 3.1: (a) Um grafo $G$ onde as linhas sólidas mostram um subgrafo induzido $H$. (b) Uma moldura de $H$ em $G$.

Nem todo subgrafo induzido planar de um grafo $G$ possui uma moldura em $G$ (figura 3.2). Na verdade, nem todo subgrafo induzido de um grafo planar $G$ possui uma moldura em $G$ (figura 3.3).

A relação entre planaridade e molduras, que será fundamental para a descrição do algoritmo de LEC, está no seguinte lema. 


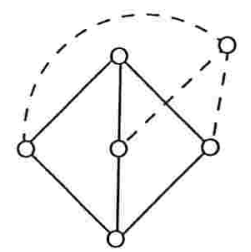

(a)

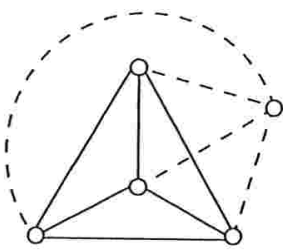

(b)

Figura 3.2: As linhas sólidas ilustram dois subgrafos induzidos de (a) $K_{3,3}$ e (b) $K_{5}$ para os quais não existe moldura (em $K_{3,3}$ e $K_{5}$, respectivamente).

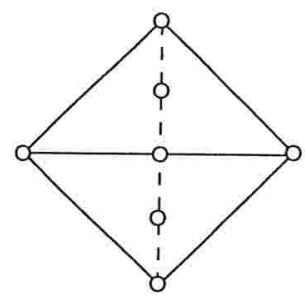

Figura 3.3: As linhas sólidas mostram um subgrafo induzido de um grafo $G$ para o qual não existe moldura em $G$.

Lema 3.1 (das molduras): Se $H$ é um subgrafo induzido de um grafo planar $G$ tal que $G-V_{H}$ é conexo, então $H$ possui uma moldura em $G$.

Demonstração: Considere um desenho plano de $G$. Este desenho induz um desenho plano de $H$. Seja $M$ o subgrafo de $H$ induzido pelas suas arestas adjacentes a face externa do desenho. Como $G-V_{H}$ é conexo, então todo vértice de $H$ vizinho de um vértice de $V_{G} \backslash V_{H}$ é vértice de $M$. Logo, $M$ é uma moldura de $H$ em $G$.

\section{Caminhos perimetrais e $X Y$-caminhos}

Seja $M$ uma moldura de um grafo $H$. Um caminho em $M$ é chamado de perimetral. Sejam $C_{1}, C_{2}, \ldots, C_{k}$ todos os blocos de $M$ que contém pelo menos uma aresta de um caminho perimetral $P$ (para cada $i, C_{i}$ é uma aresta ou um circuito). Para $i=1,2, \ldots, k$, seja $P_{i}:=P \cap C_{i}$. Seja $\bar{P}_{i}:=C_{i} \backslash P_{i}$ se $C_{i}$ tiver mais do que uma aresta, e $\bar{P}_{i}:=P_{i}$, caso contrário. O caminho $\bar{P}:=\bar{P}_{1} \cup \bar{P}_{2} \cup \ldots \cup \bar{P}_{k}$ é o caminho complementar de $P$ (figura 3.4). Se $E_{P}=\emptyset$ então $\bar{P}:=P$. A base (span) de $P($ em $M)$ é o subgrafo $C_{1} \cup C_{2} \cup \ldots \cup C_{k}$ de $M$. Se $E_{P}=\emptyset$ então a base de $P$ é o próprio $P$.

Sejam $W$ e $Z$ subconjuntos de vértices de um grafo $G$ e $v$ um vértice de $G$. Dizemos que $v$ 


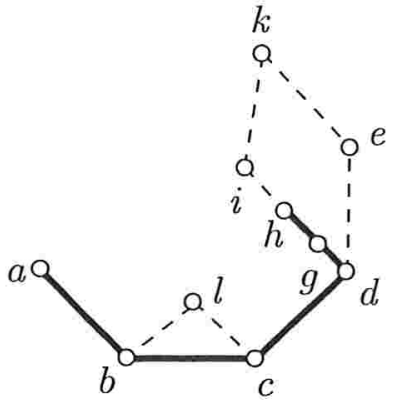

(a)

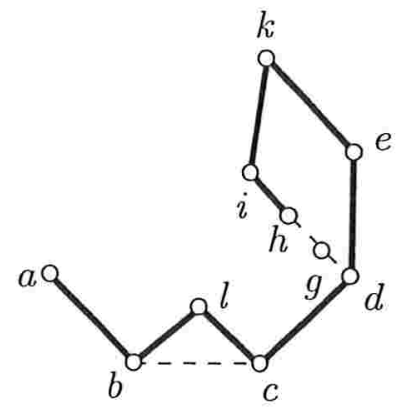

(b)

Figura 3.4: (a) Um caminho perimetral $P$ da moldura da figura 3.1. (b) O caminho complementar de $P$.

enxerga $W$ através de $Z$ se existe um caminho em $G$ de $v$ a algum vértice em $W$ com todos os seus vértices internos em $Z$. Se $Z$ é um subconjunto de arestas, então dizemos que $v$ enxerga $W$ através de $Z$ se existe um caminho em $G$ de $v$ a algum vértice em $W$ com todas as arestas em $Z$.

Seja $M$ uma moldura de um grafo conexo e sejam $X$ e $Y$ subconjuntos de $V_{M}$. Um caminho perimetral $P$ em $M$ com base $S$ é um $X Y$-caminho se:

(c1) as pontas de $P$ estão em $X$;

(c2) todo vértice de $S$ que enxerga $X$ através de $E_{M} \backslash E_{S}$ está em $P$;

(c3) todo vértice de $S$ que enxerga $Y$ através de $E_{M} \backslash E_{S}$ está em $\bar{P}$;

(c4) nenhum componente de $M-V_{S}$ contém vértices em $X$ e em $Y$.

Veja a figura 3.5.

\section{$X Y$-obstruções}

Dados uma moldura $M$ de um grafo conexo e subconjuntos $X$ e $Y$ de $V_{M}$, nem sempre é possível obter-se um $X Y$-caminho em $M$. Existem três tipos de objetos que tornam impossível a existência de um $X Y$-caminho, as chamadas $X Y$-obstruções em $M$ :

(o1) uma quíntupla $\left(C, v_{1}, v_{2}, v_{3}, v_{4}\right)$ onde $C$ é um bloco de $M$ e $v_{1}, v_{2}, v_{3}$ e $v_{4}$ são vértices distintos em $C$ e que aparecem nesta ordem em $C$, tais que $v_{1}$ e $v_{3}$ enxergam $X$ através de $E_{M} \backslash E_{C}$ e $v_{2}$ e $v_{4}$ enxergam $Y$ através de $E_{M} \backslash E_{C}$; 


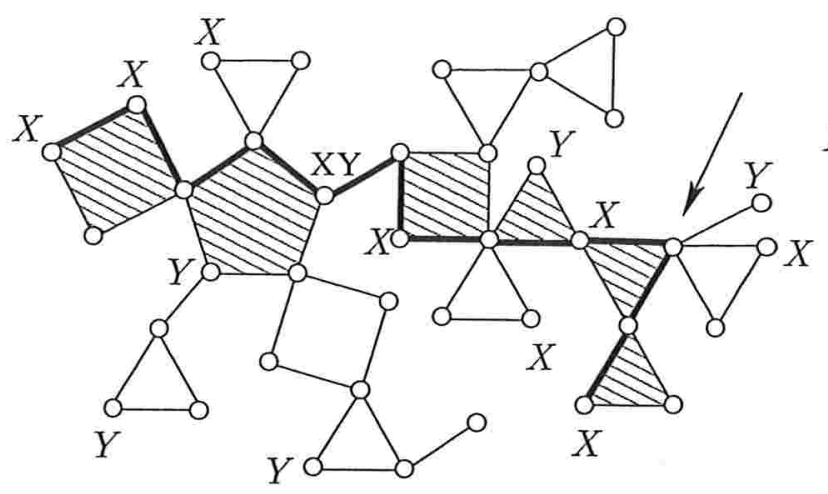

(a)

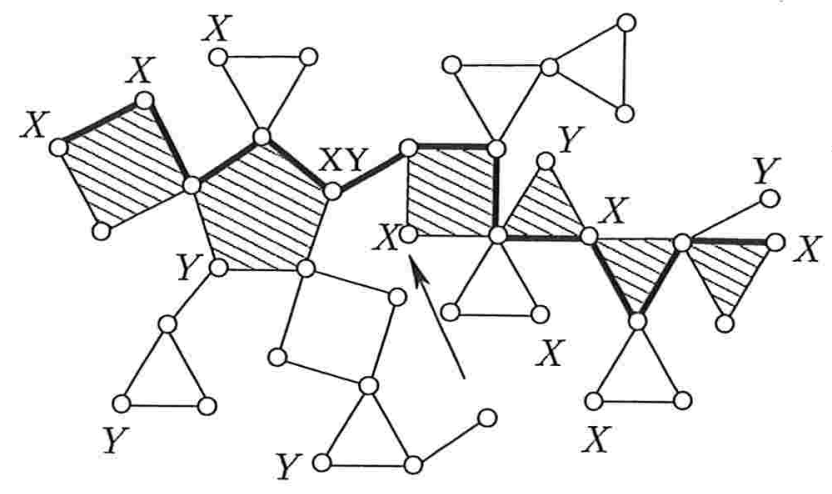

(c)

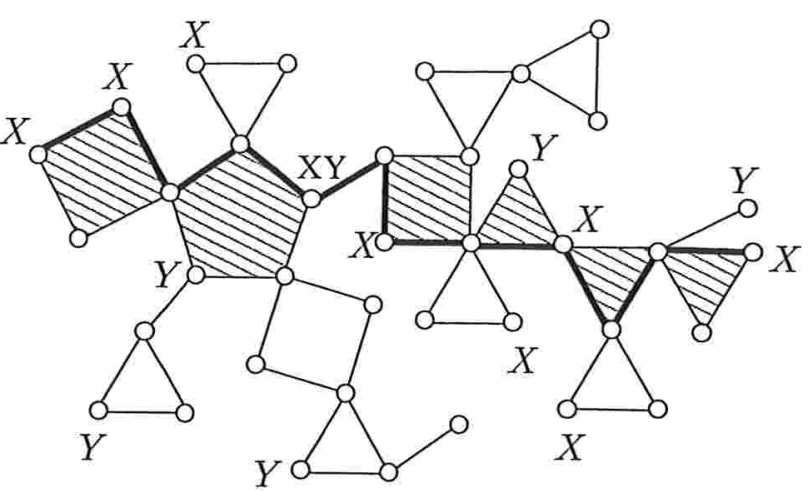

(b)

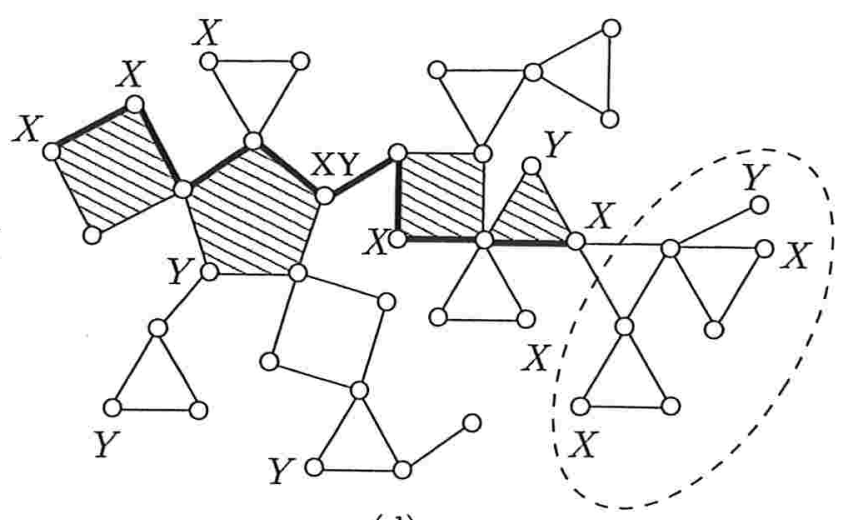

(d)

Figura 3.5: Em (a), (b), (c) e (d), denota-se por $P$ o caminho ilustrado em linhas grossas e os blocos da base do respectivo caminho estão hachurados. (a) $P$ não é um $X Y$-caminho pois viola (c3). (b) $P$ é um $X Y$-caminho. (c) $P$ não é um $X Y$-caminho pois viola (c2). (d) $P$ não é um $X Y$-caminho pois viola (c4). 
(o2) uma quádrupla $\left(C, v_{1}, v_{2}, v_{3}\right)$ onde $C$ é um bloco de $M$ e $v_{1}, v_{2}$ e $v_{3}$ são vértices distintos em $C$ que enxergam $X$ e $Y$ através de $E_{M} \backslash E_{C}$;

(o3) uma quádrupla $\left(v, K_{1}, K_{2}, K_{3}\right)$ tal que $v \in V_{M}$, e $K_{1}, K_{2}$ e $K_{3}$ são três componentes distintos de $M-v$, tais que $K_{i}$ contém vértices em $X$ e em $Y$.

\section{$X Y$-caminhos versus $X Y$-obstruções}

Como é mostrado a seguir, $X Y$-caminhos e $X Y$-obstruções são objetos que não podem coexistir.

Lema 3.2 (da $X Y$-obstrução): Seja $M$ uma moldura de um grafo e sejam $X$ e $Y$ subconjuntos de $V_{M}$. Se existe uma $X Y$-obstrução em $M$, então não existe um $X Y$-caminho em $M$.

Demonstração: Seja $\left(C, v_{1}, v_{2}, v_{3}, v_{4}\right)$ uma $X Y$-obstrução do tipo (o1). Das condições (c2) e (c3), tem-se que $C$ não pode pertencer à base de um $X Y$-caminho. Já a condição (c4) implica que $C$ deve pertencer a base de todo $X Y$-caminho. Logo, uma obstrução do tipo (o1) impede a existência de um $X Y$-caminho em $M$. A demonstração de que uma obstrução do tipo (o2) impede a existência de um $X Y$-caminho é análoga.

Suponha agora que $\left(v, K_{1}, K_{2}, K_{3}\right)$ é uma obstrução do tipo (o3). A condição (c4) implica que a base de qualquer $X Y$-caminho em $M$ deve conter vértices em cada um dos componentes $K_{1}, K_{2}$ e $K_{3}$, um absurdo.

Lema 3.3 (do $X Y$-caminho): Seja $M$ uma moldura de um grafo conexo $H$ e sejam $X$ e $Y$ subconjuntos de $V_{M}$. Se não existe uma $X Y$-obstrução em $M$, então existe um $X Y$-caminho em $M$.

Demonstração: A demonstração é por indução no número de blocos de $M$. Suponha que $M$ tem apenas um bloco $C$. Se $X=\emptyset$ então defina $P:=\emptyset$. Se $X \neq \emptyset$ então existe um caminho $P$ com pontas em $X$ que contém todos os vértices de $X$ e que não contém vértices internos em $Y$, pois, caso contrário, $C$ ou teria vértices distintos $v_{1}, v_{2}, v_{3}$ e $v_{4}$ tais que $\left(C, v_{1}, v_{2}, v_{3}, v_{4}\right)$ é uma $X Y$-obstrução do tipo (o1), ou teria vértices distintos $v_{1}, v_{2}$ e $v_{3}$ tais que $\left(C, v_{1}, v_{2}, v_{3}\right)$ é uma $X Y$-obstrução do tipo (o2). Assim, $P$ é um $X Y$-caminho em $M$.

Suponha então que $M$ possui pelo menos dois blocos. O restante da demonstração apóia-se na demonstração de alguns fatos.

Fato 3.4: Se vé um vértice de corte de $M$, então podemos supor que cada componente de $M-v$ possui um elemento de $X \cup Y$. 
Demonstração: Seja $K$ um componente de $M-v$ tal que $V_{K} \cap(X \cup Y)=\emptyset$. Seja $M^{\prime}:=$ $M-V_{K} . M^{\prime}$ é moldura de um subgrafo induzido conexo de $H$. Ademais, $M^{\prime}$ não possui uma $X Y$-obstrução; toda $X Y$-obstrução em $M^{\prime}$ é uma $X Y$-obstrução em $M$. Logo, por indução, existe um $X Y$-caminho $P$ em $M^{\prime}$. Pode-se verificar que $P$ é um $X Y$-caminho em $M$.

Fato 3.5: Se v é um vértice de corte de $M$, então podemos supor que cada componente de $M-v$ possui um elemento de $X$.

Demonstração: Seja $K$ um componente de $M-v$ tal que $V_{K} \cap X=\emptyset$. Pelo fato 3.4, podemos supor que $V_{K} \cap Y \neq \emptyset$. Sejam $M^{\prime}:=M-V_{K}$ e $Y^{\prime}:=Y \cup\{v\}$. $M^{\prime}$ é moldura de um subgrafo induzido conexo de $H$. Ademais, $M^{\prime}$ não possui uma $X Y^{\prime}$-obstrução, já que toda $X Y^{\prime}$-obstrução em $M^{\prime}$ induz uma $X Y$-obstrução em $M$. Assim, por indução, existe um $X Y^{\prime}$-caminho $P$ em $M^{\prime}$. Não é difícil ver que $P$ é um $X Y$-caminho em $M$.

Fato 3.6: Se v é um vértice de corte de $M$, então podemos supor que cada componente de $M-v$ possui um elemento de $Y$.

Demonstração: Seja $K$ um componente de $M-v$ tal que $V_{K} \cap Y=\emptyset$. Sejam $M^{\prime}:=M-V_{K}$ e $M^{\prime \prime}:=K+v . M^{\prime}$ e $M^{\prime \prime}$ são molduras de subgrafos induzidos conexos de $H$ que têm apenas o vértice $v$ em comum. Pelo fato 3.5, podemos supor que ambos, $V_{K}$ e $V_{M} \backslash\left(V_{K} \cup\{v\}\right)$ têm um elemento de $X$. Sejam $X^{\prime}:=\left(X \cap V_{M^{\prime}}\right) \cup\{v\}, X^{\prime \prime}:=\left(X \cap V_{K}\right) \cup\{v\}$. Seja $Y^{\prime \prime}:=\{v\}$ se $Y \cap V_{M^{\prime}} \neq \emptyset$, e $Y^{\prime \prime}:=\emptyset$, caso contrário. Toda $X^{\prime} Y$-obstrução em $M^{\prime}$ induz uma $X Y$-obstrução em $M$. Logo, por indução, existe um $X^{\prime} Y$-caminho $P^{\prime}$ em $M^{\prime}$. Podemos supor que $v$ é ponta de $P^{\prime}$ e que $v$ não é um elemento de $X$, pois, caso contrário, pode-se verificar que $P^{\prime}$ é um $X Y$-caminho em $M$. Analogamente, existe um $X^{\prime \prime} Y^{\prime \prime}$-caminho $P^{\prime \prime}$ em $M^{\prime \prime}$ que tem $v$ como uma de suas pontas. Finalmente, não é difícil verificar que, nessas condições, o caminho $P:=P^{\prime} \cup P^{\prime \prime}$ é um $X Y$-caminho em $M$.

Fato 3.7: Se v é um vértice de corte de $M$, então podemos supor que $M-v$ tem exatamente dois componentes.

Demonstração: Suponha que $M-v$ tem componentes distintos $K_{1}, K_{2}$ e $K_{3}$. Pelos fatos 3.5 e 3.6 podemos supor que $K_{i}$ possui um elemento de $X$ e um elemento de $Y$, para $i=1,2,3$. Portanto, $\left(v, K_{1}, K_{2}, K_{3}\right)$ é uma $X Y$-obstrução do tipo (o3), uma contradição.

Fato 3.8: Se $C$ é um bloco de $M$, então podemos supor que $M-V_{C}$ tem no máximo dois componentes.

Demonstração: Suponha que $M-V_{C}$ tem componentes distintos $K_{1}, K_{2}$ e $K_{3}$. Pelos fatos 3.5 e 3.6 tem-se que $K_{i}$ possui um vértice de $X$ e um vértice de $Y, i=1,2,3$. Seja $v_{i}$ o vértice de $C$ 
que tem vizinhos em $K_{i}, i=1,2,3$. Pelo fato 3.7 podemos supor que $v_{1}, v_{2}$ e $v_{3}$ são vértices distintos. Pelos fatos 3.5 e 3.6 tem-se que cada $v_{i}$ enxerga $X$ e $Y$ através de $E_{M} \backslash E_{C}$. Portanto, $\left(C, v_{1}, v_{2}, v_{3}\right)$ é uma $X Y$-obstrução do tipo (o2), uma contradição.

Agora, estamos preparados para concluir a demonstração do lema 3.3. Pelos fatos 3.7 e 3.8, podemos supor que os blocos $C_{1}, \ldots, C_{k}$ de $M$ são tais que

se $C_{i}$ e $C_{j}$ têm um vértice em comum então $|i-j| \leq 1$.

Sejam $v$ um vértice de corte de $M$ e $K$ um componente de $M-v$. Seja $M^{\prime}$ o grafo $M-V_{K}$ e $M^{\prime \prime}$ o subgrafo de $M$ induzido por $V_{K} \cup\{v\}$. $M^{\prime}$ e $M^{\prime \prime}$ são molduras de subgrafos induzidos conexos de $H$. Pelos fatos 3.5 e 3.6 tem-se que $M^{\prime}$ e $M^{\prime \prime}$ têm elementos de $X$ e $Y$. Sejam $X^{\prime}:=\left(X \cap V_{M^{\prime}}\right) \cup\{v\}, Y^{\prime}:=\left(Y \cap V_{M^{\prime}}\right) \cup\{v\}, X^{\prime \prime}:=\left(X \cap V_{M^{\prime \prime}}\right) \cup\{v\}$ e $Y^{\prime \prime}:=\left(Y \cap V_{M^{\prime \prime}}\right) \cup\{v\}$. Toda $X^{\prime} Y^{\prime}$-obstrução em $M^{\prime}$ induz uma $X Y$-obstrução em $M$. Logo, por indução, existe um $X^{\prime} Y^{\prime}$-caminho $P^{\prime}$ em $M^{\prime}$. Como $v$ está em $X^{\prime} \cap Y^{\prime}$ e devido a (3.1) tem-se que $v$ é uma ponta de $P^{\prime}$. De maneira similar, existe um $X^{\prime \prime} Y^{\prime \prime}$-caminho $P^{\prime \prime}$ com ponta $v$ em $M^{\prime \prime}$. Pode-se verificar que $P^{\prime} \cup P^{\prime \prime}$ é um $X Y$-caminho em $M$.

O teorema abaixo resume o conteúdo dos lemas 3.2 e 3.3 da maneira conveniente para a descrição do algoritmo de LEC.

Teorema 3.9: Seja $M$ uma moldura de um grafo conexo e sejam $X$ e $Y$ subconjuntos de $V_{M}$. Vale uma e apenas uma das seguintes afirmações:

(1) existe uma $X Y$-obstrução em $M$;

(2) existe um $X Y$-caminho em $M$.

\subsection{Lec-ordenação}

Um dos ingredientes importantes do algoritmo de LEC é uma certa ordenação $v_{1}, v_{2}, \ldots, v_{n}$ dos vértices de um grafo $G$ tal que, para $i=1, \ldots, n$, os subgrafos

$$
G\left[\left\{v_{1}, \ldots, v_{i}\right\}\right] \text { e } G\left[\left\{v_{i+1}, \ldots, v_{n}\right\}\right] \text { são ambos conexos. }
$$

Equivalentemente, $G$ é conexo e, para $i=2, \ldots, n-1$, tem-se que $v_{i}$ é adjacente a vértices $v_{j}$ e $v_{k} \operatorname{com} 1 \leq j<i<k \leq n$. 


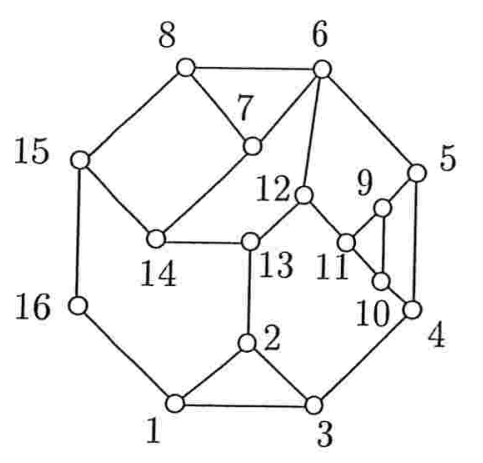

(a)

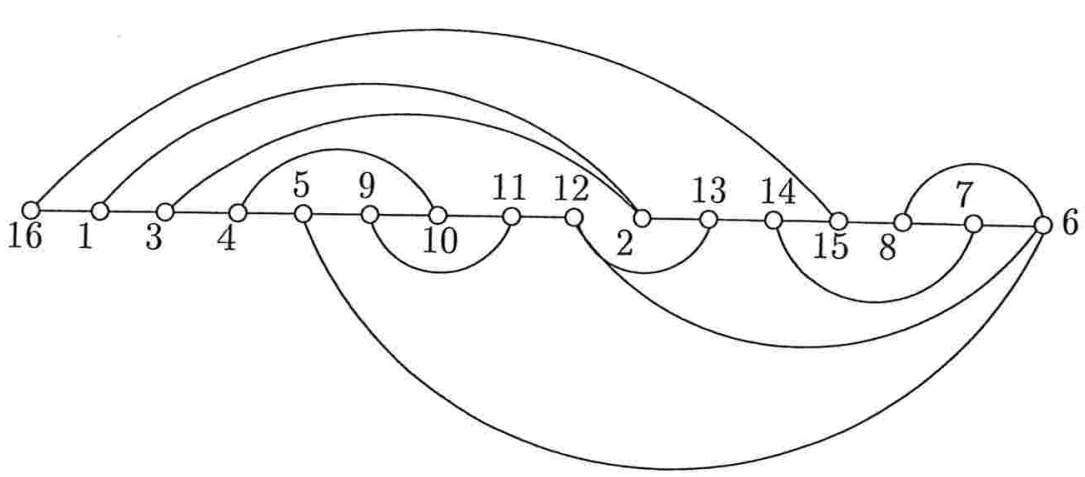

(b)

Figura 3.6: (a) Um grafo $G$. (b) Uma lec-ordenação de $G$.

Uma tal ordenação é dita uma lec-ordenação de $G$ (figura 3.6). Uma lec-ordenação onde $v_{1} v_{n}$ é uma aresta do grafo é conhecida como st-ordenação [12]. A numeração dos vértices do grafo da figura 3.6 é uma st-ordenação.

Nem todo grafo possui uma lec-ordenação, como mostra a figura 3.7. Entretanto, é possível demonstrar que todo grafo biconexo possui uma lec-ordenação.

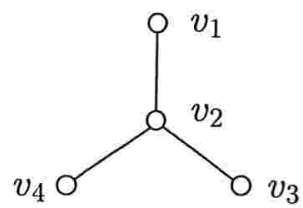

Figura 3.7: Um grafo que não possui uma lec-ordenação.

Lema 3.10 (da lec-ordenação): Todo grafo biconexo possui uma lec-ordenação.

Demonstração: Por indução no número de vértices do grafo. O lema vale para grafos com no máximo dois vértices. Assim, seja $G=(V, E)$ um grafo com $n$ vértices, $n>2$. Seja $u v$ a aresta em uma das pontas de um caminho maximal $P$ em $G$. Seja $x$ um elemento que não está em $V$. Sejam

$$
V^{\prime}:=(V \backslash\{u, v\}) \cup\{x\} \quad \text { e } \quad E^{\prime}:=(E \backslash\{u v\}) \cup\{x w: u w \in E \text { ou } v w \in E\} .
$$

Como $G$ é biconexo, então da maximalidade de $P$ tem-se que $G-\{u, v\}$ é conexo. Portanto $G^{\prime}=\left(V^{\prime}, E^{\prime}\right)$ é biconexo. É dito que $G^{\prime}$ corresponde ao grafo obtido de $G$ pela contração da aresta $u v$ (e pela remoção de eventuais "arestas paralelas"). Por indução, $G^{\prime}$ possui uma 
lec-ordenação $v_{1}^{\prime}, v_{2}^{\prime}, \ldots, v_{n-1}^{\prime} \operatorname{com} v_{i}^{\prime}=x$ para algum $i$ em $\{1,2, \ldots, n-1\}$. Trocando $x$ na lec-ordenação de $G^{\prime}$ por $u, v$ ou $v, u$ obtém-se uma lec-ordenação de $G$.

\subsection{Descrição do algoritmo}

Dado um grafo biconexo $G$, o algoritmo de LEC examina os vértices de $G$ um após o outro de acordo com uma lec-ordenação. Em cada iteração, o algoritmo procura estender uma moldura do subgrafo induzido pelos vértices já examinados. Caso isso não seja possível, o algoritmo declara que o grafo não é planar e pára.

Algoritmo de $\operatorname{LEC}(G)$. Recebe um grafo biconexo $G$ e devolve sim se $G$ é planar, e NÃO, caso contrário.

Cada iteração começa com um subgrafo induzido $H$ de $G$, uma moldura $M$ de $H$ em $G$, e uma lista $L$ dos vértices do grafo $G-V_{H}$.

No início da primeira iteração tem-se $H=\emptyset, M=\emptyset$ e $L$ é uma lec-ordenação de $G$. Cada iteração consiste no seguinte:

Caso 1: $L=\emptyset$.

Devolva SIM e pare.

Caso 2: $L \neq \emptyset$.

Seja $w$ o primeiro vértice na lista $L$.

Seja $L^{\prime}$ a lista resultante após a remoção de $w$ de $L$.

Seja $X:=\left\{u \in V_{M}: u w \in E_{G}\right\}$.

Seja $Y:=\left\{u \in V_{M}:\right.$ existe $\left.v \in V_{G} \backslash\left(V_{H} \cup\{w\}\right), u v \in E_{G}\right\}$.

Caso 2A: Existe uma $X Y$-obstrução em $M$.

Devolva NÃO e pare.

Caso 2B: Existe um $X Y$-caminho $P$ em $M$.

Seja $\bar{P}:=\left\langle w_{0}, w_{1}, \ldots, w_{k}\right\rangle$ o caminho complementar de $P$.

Seja $R$ o conjunto dos vértices em $V_{M} \backslash V_{\bar{P}}$ que não enxergam $Y \backslash V_{\bar{P}}$ através de $V_{M} \backslash V_{\bar{P}}$ (figura 3.8(b)).

Seja $M^{\prime}$ o grafo resultante após acrescentar-se o vértice $w$ e as arestas $w w_{0}$ e $w w_{k}$ ao grafo $M-R$ (figura 3.8(d)).

Seja $H^{\prime}:=H+w$ (figura $3.8(\mathrm{c})$ ).

Comece nova iteração com $H^{\prime}, M^{\prime}$ e $L^{\prime}$ nos papéis de $H, M$ e $L$ respectivamente. 


\subsection{Invariantes}

A correção do algoritmo de LEC baseia-se nas demonstrações da validade de uma série de invariantes. Estes invariantes são afirmações envolvendo o grafo dado e os objetos $H, M$ e $L$. As afirmações são válidas no início de cada iteração do algoritmo e dizem como estes objetos se relacionam entre si e com os dados do problema. As seguintes afirmações são invariantes do algoritmo de LEC:

(lec1) $H$ e $G-V_{H}$ são grafos conexos;

(lec2) $M$ é uma moldura de $H$ em $G$.

Para demonstrar-se que as afirmações acima são legítimos invariantes deve-se verificar que:

(a) as afirmações valem no início da primeira iteração e

(b) se as afirmações valem no início de uma iteração em que ocorre o caso $2 \mathrm{~B}$, então as afirmações também valem no final da iteração com $H^{\prime}, M^{\prime}$ e $L^{\prime}$ nos papéis de $H, M$ e $L$, respectivamente.

De (a) e (b) conclui-se que (lec1) e (lec2) também valem no início da última iteração, quando ocorre o caso 1 ou o caso $2 \mathrm{~A}$.

É evidente que as afirmações valem no início da primeira iteração e não é difícil verificar (b) para (lec1). A seguir está a demonstração de (b) para (lec2).

Demonstração de (lec2): Considere uma iteração em que ocorre o caso 2B. No início de cada iteração tem-se que $H$ e $G-V_{H}$ são grafos conexos (invariante (lec1)) e que $M$ é uma moldura de $H$ em $G$ (invariante (lec2)). Note que, de (m2) na definição de moldura, vale que

$$
X=\left\{u \in V_{H}: u w \in E_{G}\right\} \quad \text { e } \quad Y=\left\{u \in V_{H} \text { : existe } v \in V_{G} \backslash\left(V_{H} \cup\{w\}\right), u v \in E_{G}\right\} .
$$

Considere um desenho plano $D$ de $H$ correspondente à moldura $M$ (figura 3.8(a)). Seja $S$ a base do $X Y$-caminho $P$ em $M$ determinado na iteração. Podemos supor que os caminhos $P$ e $\bar{P}$ não se "cruzam" (figura 3.8(b)).

Pela definição de $R$ (vértices marcados com quadrados na figura 3.8(b)), tem-se que não existe aresta com uma ponta em $R$ e outra em $V_{G} \backslash\left(R \cup V_{\bar{P}} \cup\{w\}\right)$. Além disso, da condição (c2) na definição de $X Y$-caminho, vale que todo vizinho de $w$ em $V_{H}$ está em $V_{P} \cup R$. Logo, um desenho 
plano $D^{\prime}$ de $H^{\prime}$ pode ser obtido acrescentando-se o vértice $w$ e as arestas de $E_{G}$ com uma ponta em $X$ e outra em $w$. Para isto, basta acrescentar $w$ ao desenho $D$ de tal forma que o circuito formado pelas arestas $w w_{0}$ e $w w_{k}$ e pelo caminho $P$ tem em seu interior somente vértices de $R$ (figura 3.8(c)).

O grafo $M^{\prime}$ definido pelo algoritmo é uma moldura de $H^{\prime}$ em $G$ correspondente ao desenho $D^{\prime}$ (figura 3.8(d)). De fato, $M^{\prime}$ é a moldura de $H^{\prime}$ correspondente ao desenho $D^{\prime}$. Ademais, de (c3) na definição de $X Y$-caminho, tem-se que todo vértice de $H^{\prime}$ que é vizinho de um vértice de $V_{G} \backslash V_{H^{\prime}}$ é um vértice de $M^{\prime}$. Logo, $M^{\prime}$ é uma moldura de $H^{\prime}$ que satisfaz (m2). Portanto, $M^{\prime}$ é uma moldura de $H^{\prime}$ em $G$.

\subsection{Correção}

A correção do algoritmo de LEC é facilmente demonstrada através dos invariantes apresentados e do lema a seguir.

Lema 3.11: Seja $M$ a moldura no início de uma iteração do algoritmo de LEC em que ocorre o caso 2. Se existe uma $X Y$-obstrução em $M$ então $G$ possui um subgrafo de Kuratowski.

Demonstração: Pelo invariante (lec1), tem-se que $G-V_{H}$ é conexo. Seja $T$ uma árvore geradora de $G-V_{H}$. Inicialmente $L$ é uma lec-ordenação dos vértices de $G$, logo, devido a (3.2), podemos supor que $w$ é uma folha de $T$. Logo, $G-\left(V_{H} \cup\{w\}\right)$ é um grafo conexo.

Existem três possibilidades a serem consideradas dependendo do tipo da $X Y$-obstrução encontrada no caso $2 \mathrm{~A}$. Verificaremos que cada uma dessas possibilidades implica na existência de um subgrafo de Kuratowski em $G$.

(1) Existe uma $X Y$-obstrução $\left(C, v_{1}, v_{2}, v_{3}, v_{4}\right)$ do tipo (o1) em $M$.

Pela definição de $X Y$-obstrução do tipo (o1), existem caminhos internamente disjuntos $P_{1}, P_{2}, P_{3}$ e $P_{4}$ com todos os vértices internos em $V_{M} \backslash V_{C}$, tais que:

(1a) $P_{1}$ é um caminho entre $v_{1}$ e $w . P_{3}$ é um caminho entre $v_{3}$ e $w$. Todas as arestas de $P_{1}$ e $P_{3}$ estão em $E_{M}$, exceto as com ponta em $w$;

(1b) $P_{2}$ é um caminho entre $v_{2}$ e um vértice $u_{2}$ em $V_{G} \backslash\left(V_{H} \cup\{w\}\right)$. $P_{4}$ é um caminho entre $v_{4}$ e um vértice $u_{4}$ em $V_{G} \backslash\left(V_{H} \cup\{w\}\right)$. Todas as arestas de $P_{2}$ e $P_{4}$ estão em $E_{M}$, exceto as com ponta em $u_{2}$ e $u_{4}$.

Seja $T^{\prime}$ a subárvore de $T$ com folhas em $\left\{u_{2}, u_{4}, w\right\}$. Pode-se verificar que o subgrafo de $G$ formado por $C \cup P_{1} \cup P_{2} \cup P_{3} \cup P_{4} \cup T^{\prime}$ é uma subdivisão de $K_{3,3}$ (figura 3.9). 


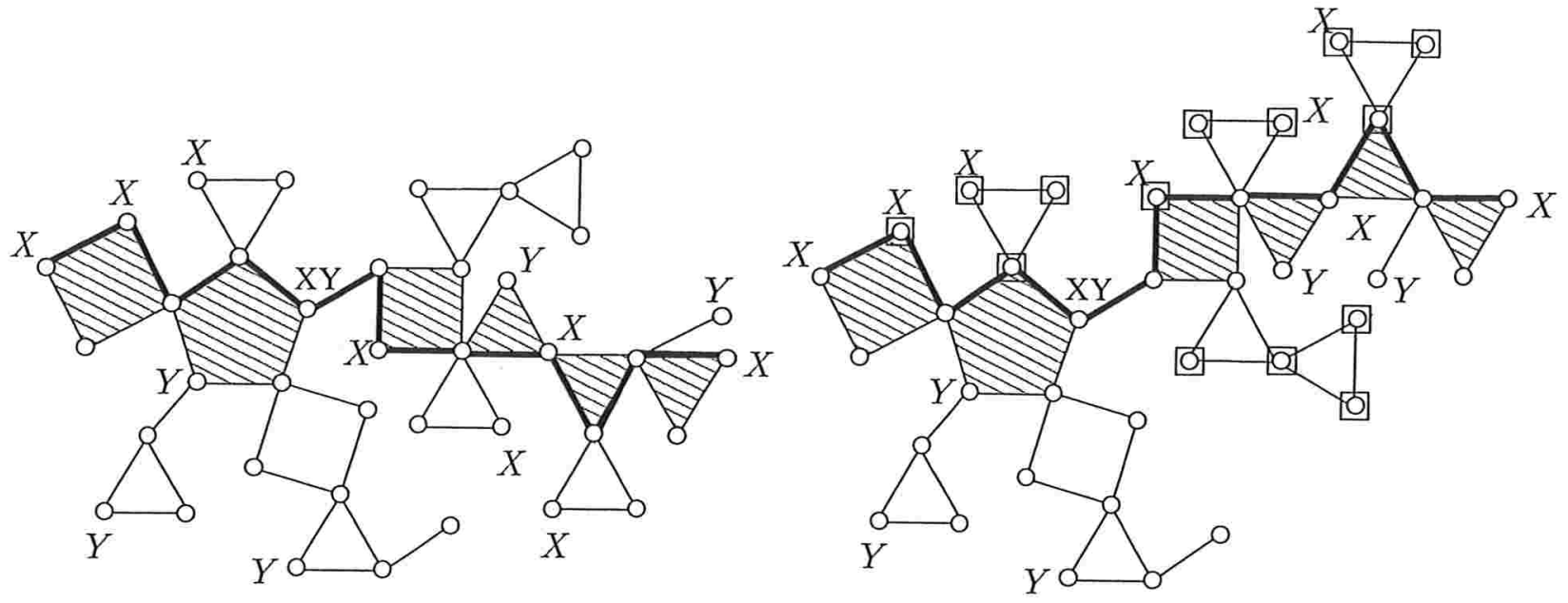

(a)

(b)

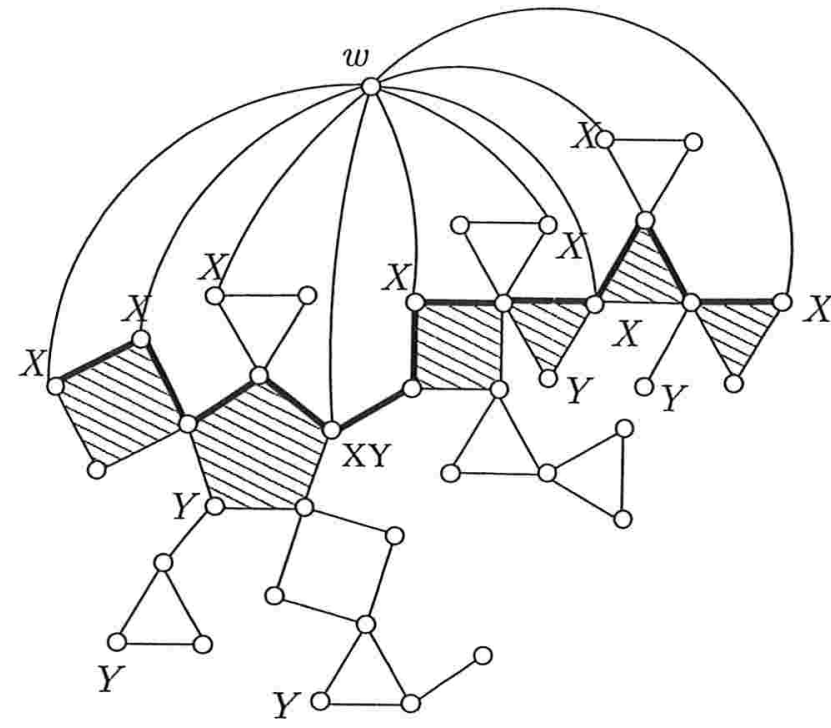

(c)

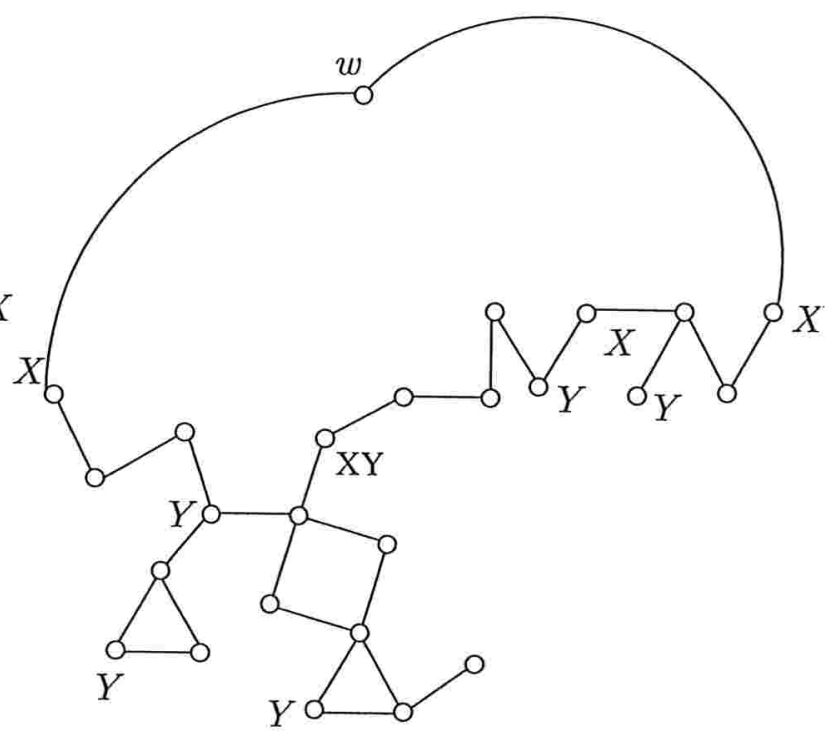

(d)

Figura 3.8: (a) Uma moldura $M$ para $H$ e um $X Y$-caminho $P$ (ilustrado em linhas grossas). (b) $M$ após deixar os elementos de $X$ de um lado e os de $Y$ de outro em relação a $P$. Os vértices em $V_{M} \backslash V_{\bar{P}}$ que não enxergam $Y \backslash V_{\bar{P}}$ através de $V_{M} \backslash V_{\bar{P}}$ estão marcados com quadrados, onde $\bar{P}$ é o caminho complementar de $P$. (c) $M$ juntamente com as arestas com uma ponta em $M$ e outra em $w$. (d) Uma moldura para $H+w$. 
(2) Existe uma $X Y$-obstrução $\left(C, v_{1}, v_{2}, v_{3}\right)$ do tipo (o2) em $M$.

Existem caminhos $P_{1}, P_{2}, P_{3}, Q_{1}, Q_{2}, Q_{3}$ com todos os vértices internos em $V_{M} \backslash V_{C}$, tais que:

(2a) Para $i=1,2,3, Q_{i}$ é um caminho entre $v_{i}$ e $w$ e todas as arestas de $Q_{i}$ estão em $E_{M}$, exceto a com ponta em $w$;

(2b) Para $i=1,2,3, P_{i}$ é um caminho entre $v_{i}$ e um vértice $u_{i}$ em $V_{G} \backslash\left(V_{H} \cup\{w\}\right)$ e todas as arestas de $P_{i}$ estão em $E_{M}$, exceto a com ponta em $u_{i}$;

(2c) Para $i=1,2,3, P_{i} \cup Q_{i}$ é uma árvore cujas folhas estão em $\left\{u_{i}, v_{i}, w\right\}$;

(2d) Para $i \neq j$, os vértices em comum de $P_{i} \cup Q_{i}$ e $P_{j} \cup Q_{j}$ estão em $\left\{w, u_{i}\right\}$.

Seja $T^{\prime}$ a subárvore de $T$ com folhas em $\left\{u_{1}, u_{2}, u_{3}, w\right\}$. Seja $O$ o subgrafo de $G$ definido por $C \cup P_{1} \cup P_{2} \cup P_{3} \cup Q_{1} \cup Q_{2} \cup Q_{3} \cup T^{\prime}$. Se, para $i=1,2,3, P_{i}$ e $Q_{i}$ são internamente disjuntos e existe um vértice em $T^{\prime}$ de grau 4 em $O$, então $O$ é uma subdivisão de $K_{5}$ (figura 3.10(a)), caso contrário, $O$ é uma subdivisão de $K_{3,3}$ (figuras 3.10(b) e 3.10 (c)).

(3) Existe uma $X Y$-obstrução $\left(v, K_{1}, K_{2}, K_{3}\right)$ do tipo (o3) em $M$.

Pela definição de $X Y$-obstrução do tipo (o3), existem caminhos $P_{1}, P_{2}, P_{3}, Q_{1}, Q_{2}, Q_{3}$ com todos os vértices internos em $V_{M}$, tais que

(3a) Para $i=1,2,3, Q_{i}$ é um caminho entre $v$ e $w$ e todas as arestas de $Q_{i}$ estão em $E_{K_{i}}$, exceto as com ponta em $w$ ou $v$;

(3b) Para $i=1,2,3, P_{i}$ é um caminho entre $v$ e um vértice $u_{i}$ em $V_{G} \backslash\left(V_{H} \cup\{w\}\right)$ e todas as arestas de $P_{i}$ estão em $E_{K_{i}}$, exceto as com ponta em $w$ ou $v$;

(3c) Para $i=1,2,3, P_{i} \cup Q_{i}$ é uma árvore com folhas $u_{i}, v$ e $w$. (Em particular $P_{i} \cap Q_{i}$ é um caminho com pelo menos uma aresta.)

(3d) Para $i \neq j$, os vértices em comum de $P_{i} \cup Q_{i}$ e $P_{j} \cup Q_{j}$ estão em $\left\{u_{i}, v, w\right\}$.

Seja $T^{\prime}$ a subárvore de $T$ com folhas em $\left\{u_{1}, u_{2}, u_{3}, w\right\}$. O subgrafo de $G$ definido por $P_{1} \cup P_{2} \cup P_{3} \cup Q_{1} \cup Q_{2} \cup Q_{3} \cup T^{\prime}$ é uma subdivisão de $K_{3,3}$ (figura 3.11).

Portanto, cada tipo de $X Y$-obstrução em $M$ implica na existência de um subgrafo de Kuratowski em $G$. Isto conclui a demostração do lema 3.11 .

Teorema 3.12 (da correção): O algoritmo de LEC decide se um dado grafo é planar.

Demonstração: Como o grafo $G$ dado é biconexo, então, pelo lema 3.10, $G$ possui uma lecordenação (calculada antes da primeira iteração do algoritmo).

Se o algoritmo pára após executar o caso 1 e devolve SIM então, da definição de moldura, o grafo dado é planar e a resposta está correta.

Suponha que o algoritmo está numa iteração em que ocorre o caso 2. Seja $M$ a moldura no início da iteração e $X$ e $Y$ os conjuntos definidos no início do caso 2. Pelo teorema 3.9, ocorre 

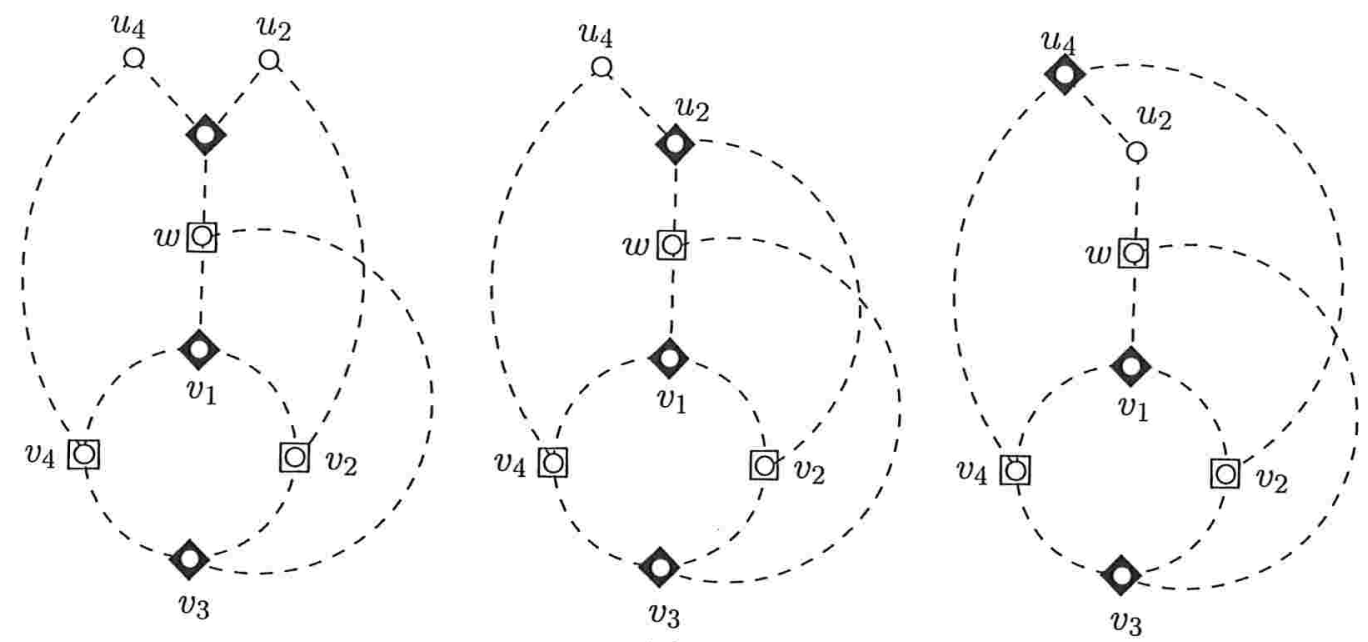

(a)

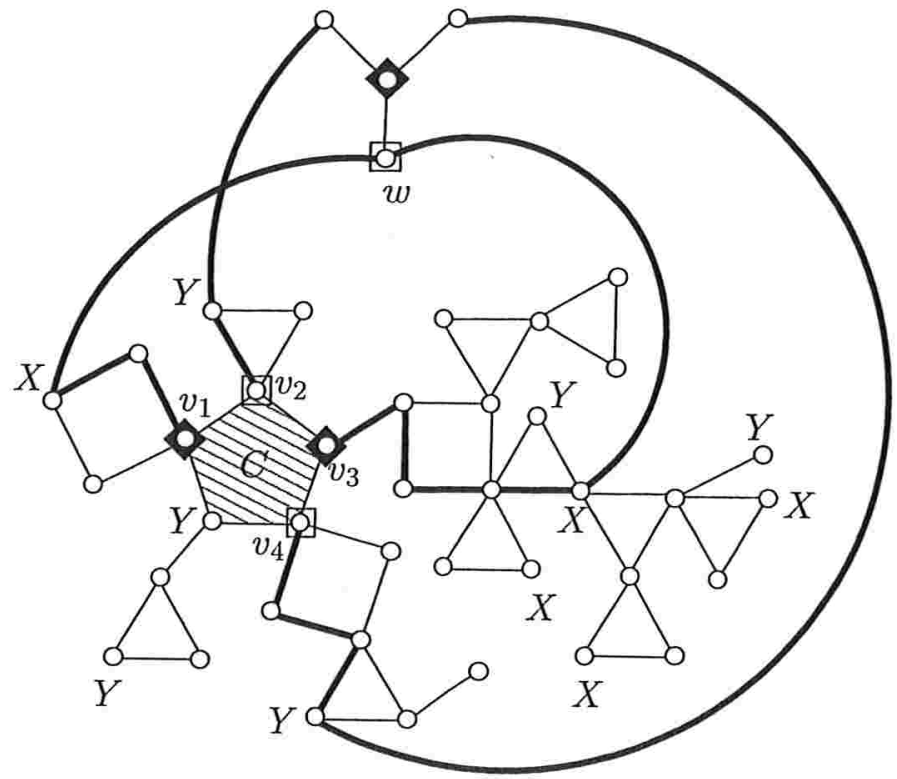

(b)

Figura 3.9: (a) Os quadrados e losangos ilustram os vértices da bipartição de uma subdivisão de $K_{3,3}$. (b) Um exemplo concreto onde pode ser vista uma subdivisão de $K_{3,3}$, como na primeira figura em (a). 


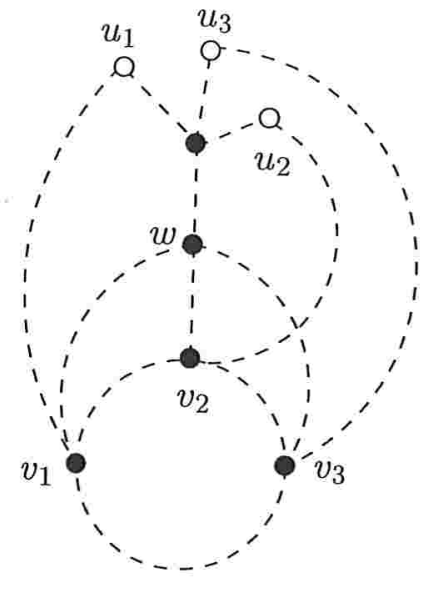

(a)

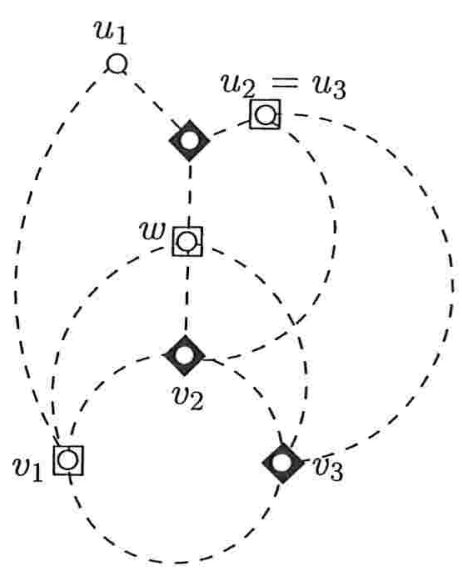

(b)

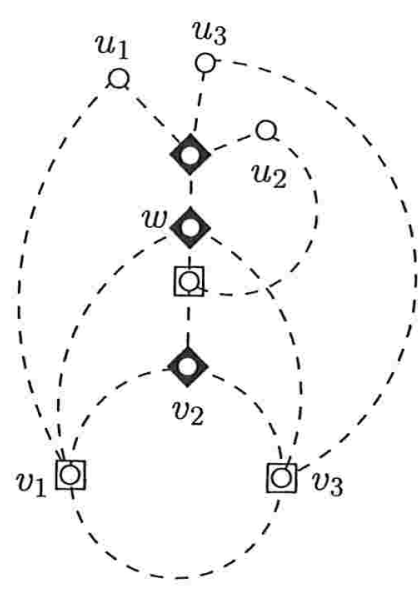

(c)

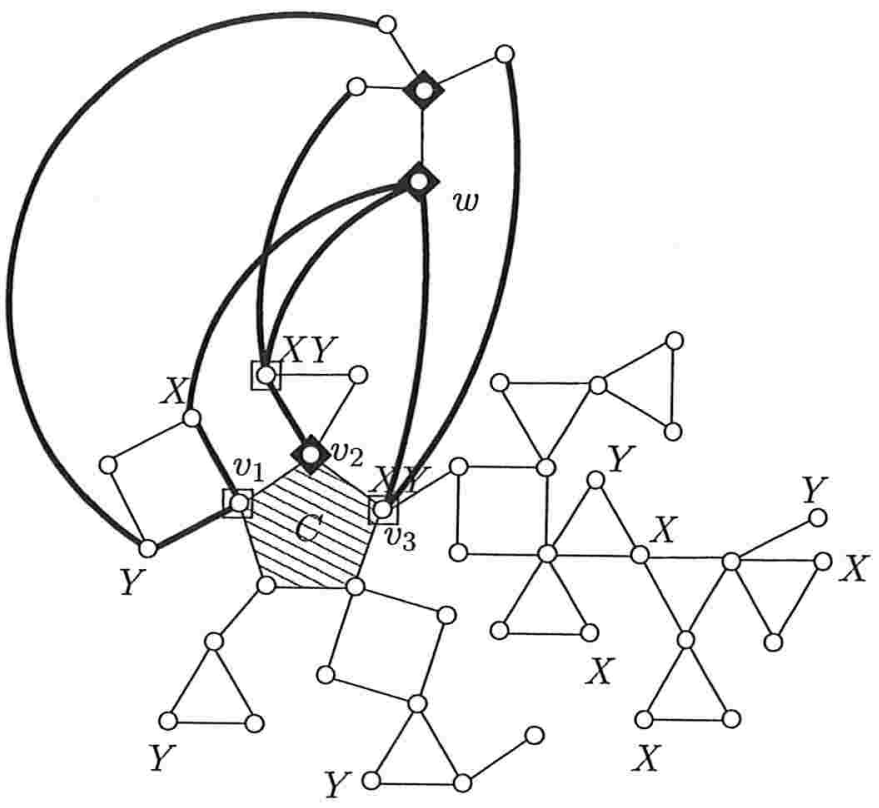

(d)

Figura 3.10: (a) Uma subdivisão de $K_{5}$. Em (b) e (c), os quadrados e losangos ilustram os vértices da bipartição de uma subdivisão de $K_{3,3}$. (d) Um exemplo concreto onde pode ser vista uma subdivisão de $K_{3,3}$, como em (c). 


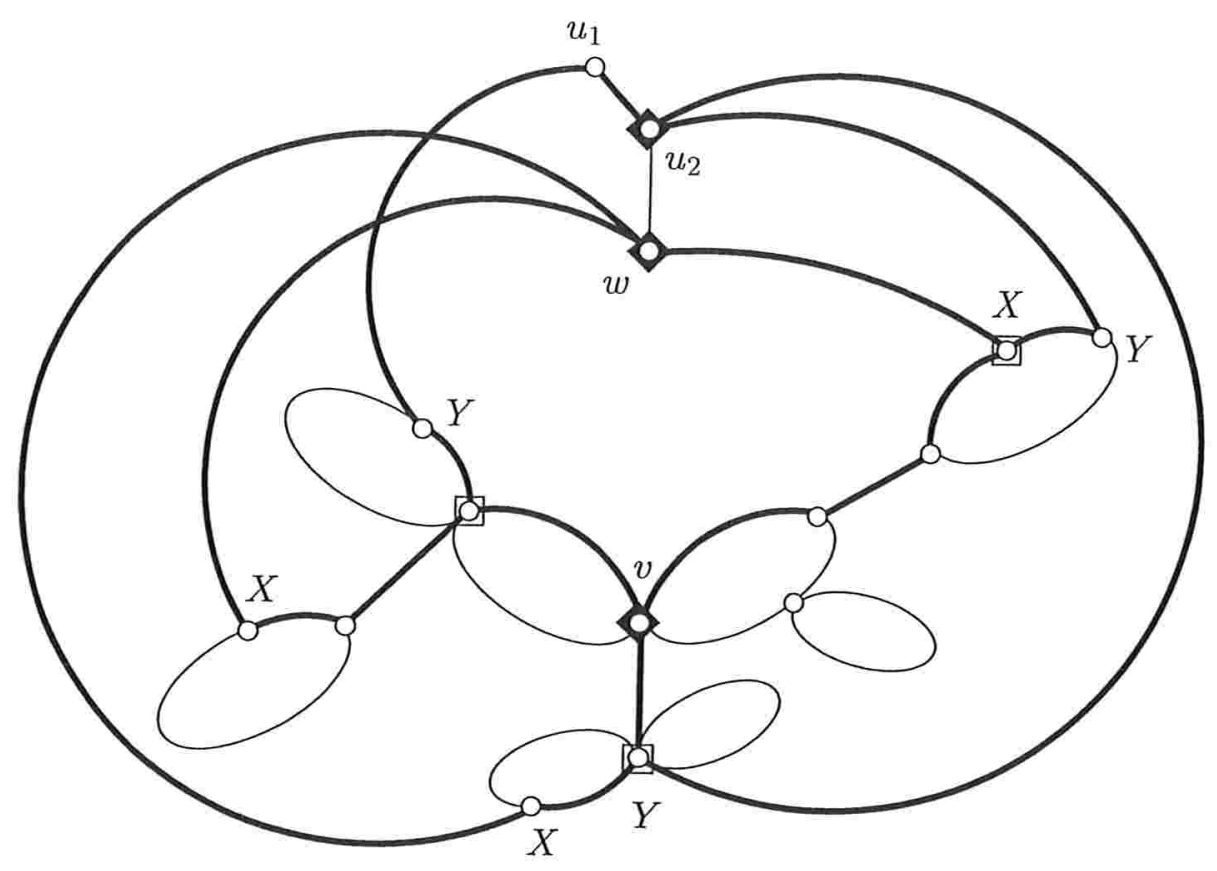

(a)

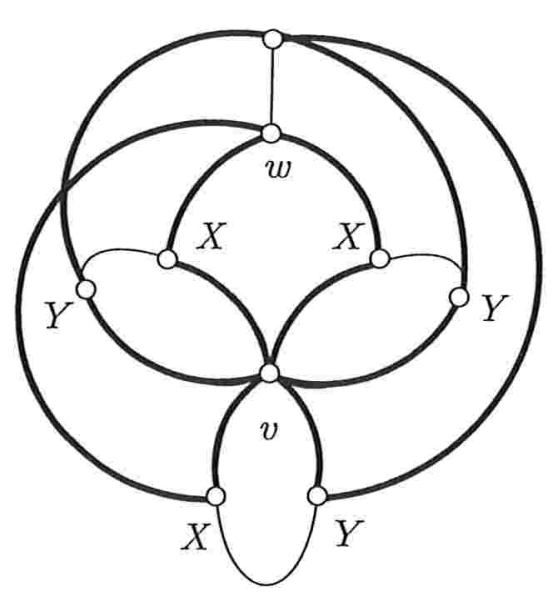

(b)

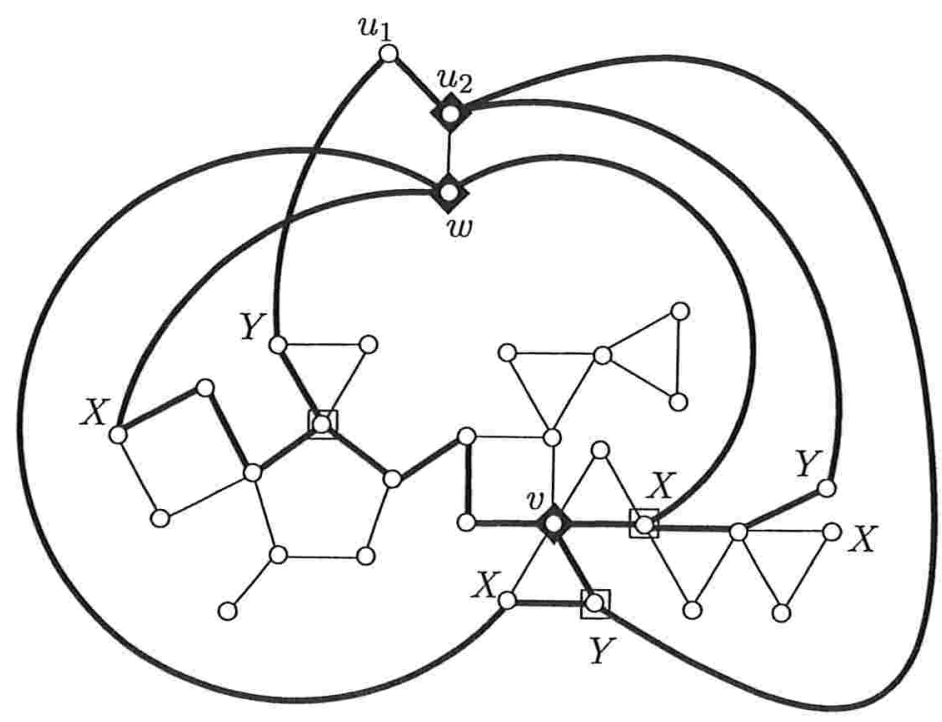

(c)

Figura 3.11: (a) Obstrução do tipo (o3). (b) Não há um subgrafo de Kuratowski que contém os caminhos em linhas grossas. (c) Exemplo concreto onde pode ser vista uma subdivisão de $K_{3,3}$, como em (a). 
um e apenas um, entre os casos $2 \mathrm{~A}$ e 2B. Se ocorre o caso 2B, então, pelos resultados da seção anterior, os invariantes (lec1) e (lec2) valem no início da próxima iteração. Se ocorre o caso 2A, então o algoritmo pára e corretamente declara o grafo como não-planar. De fato, pelo lema 3.11, $G$ possui um subgrafo de Kuratowski e portanto, pelo lema 2.6, $G$ não é planar.

Finalmente, é evidente que o algoritmo pára depois de no máximo $n$ iterações, onde $n$ é o número de vértices de $G$.

\subsection{Teorema de Kuratowski}

Uma conseqüência importante da correção do algoritmo de LEC é o bem-conhecido teorema de Kuratowski.

Corolário 3.13 (Teorema de Kuratowski): Um grafo é planar se e somente se não possui um subgrafo de Kuratowski.

Demonstração: Seja $G$ um grafo. É claro que $G$ é planar se e somente se cada um dos seus componentes biconexos é planar. Logo, podemos supor que $G$ é biconexo. Pelo lema 2.6, tem-se que se $G$ possui um subgrafo de Kuratowski, então $G$ não é planar. Reciprocamente, suponha que $G$ não é planar e considere uma aplicação do algoritmo de LEC tendo $G$ como entrada. Como $G$ não é planar, então, pelo teorema 3.12, o algoritmo pára em alguma iteração após a ocorrência do caso $2 \mathrm{~A}$, depois de encontrar uma $X Y$-obstrução na moldura corrente. Pelo lema $3.11, G$ possui um subgrafo de Kuratowski.

\subsection{Eficiência}

A eficiência do algoritmo de LEC depende do tempo consumido para resolver cada um dos seguintes subproblemas:

Problema da lec-ordenação: Dado um grafo biconexo $G$, encontrar uma lecordenação dos vértices de $G$.

Problema do $X Y$-caminho: Dados uma moldura conexa $M$ e subconjuntos $X$ e $Y$ de $V_{M}$, encontrar um $X Y$-caminho ou uma $X Y$-obstrução em $M$.

Problema da nova moldura: Dados uma moldura $M$ de um grafo conexo $H$, subconjuntos $X$ e $Y$ de $V_{M}$, um $X Y$-caminho $P$ em $M$, e um elemento $w$ que não está em $V_{H}$, construir uma moldura $M^{\prime}$ para o grafo $\left(V_{H} \cup\{w\}, E_{H} \cup\{w u: u \in X\}\right)$. 
A seguir, cada subproblema é tratado independentemente. Ao final desta seção, é apresentado o consumo de tempo resultante para o algoritmo de LEC.

\section{Determinação de uma lec-ordenação}

Seja $G$ um grafo biconexo com $n$ vértices e $m$ arestas. O lema 3.10 pode ser facilmente transformado em um algoritmo recursivo para obter uma lec-ordenação dos vértices de $G$. Se $n \leq 2$, na base da recursão, qualquer ordenação dos vértices de $G$ é uma lec-ordenação; este caso consome tempo constante. Suponha que $n>2$. Neste caso, o algoritmo, antes da chamada recursiva, precisa determinar uma aresta $u v$ que seja ponta de um caminho maximal em $G$. Depois da determinação de $u v$, o algoritmo obtém, recursivamente, uma lec-ordenação para o grafo $G^{\prime}$ resultante de $G$ após a contração da aresta $u v$. Qualquer aresta incidente a uma folha de uma árvore de busca em profundidade (bp-árvore) de $G$ é ponta de um caminho maximal em $G$. A determinação de $u v$ e a construção de $G^{\prime}$ consomem tempo $O(n+m)$.

Finalmente, dada uma lec-ordenação de $G^{\prime}$, uma lec-ordenação de $G$ é obtida trocando-se o vértice $x$ resultante da contração de $u v$ por $u, v$ ou $v, u$. A determinação de qual entre as ordens $u, v$ e $v, u$ produz uma lec-ordenação pode ser feita em tempo $O(n+m)$. O consumo de tempo do algoritmo resultante é $O\left(n^{2}+m^{2}\right)$. A seguir encontra-se uma versão iterativa do algoritmo descrito. No algoritmo, se $L=\left\langle v_{0}, v_{1}, \ldots, v_{i}, \ldots, v_{k}\right\rangle$ é uma lista, então dizemos que $v_{0}, \ldots, v_{i-1}$ são os predecessores de $v_{i}$ em $L$ e $v_{i+1}, l d o t s, v_{n}$ são os sucessores de $v_{i}$.

Algoritmo lec-ordenação $(G)$. Recebe um grafo biconexo $G$ e devolve uma lecordenação de $V_{G}$.

Cada iteração começa com duas listas $L$ e $I$ de vértices de $G$. No início da primeira iteração, $L=\emptyset$ e $I$ representa uma ordenação de $V_{G}$ obtida ao percorrer uma bp-árvore $T$ de $G$ em préordem. (A lista $L$ representa uma lec-ordenação dos vértices de $G$ após a contração das arestas de $T$ que não têm ambas as pontas em $I$.) Cada iteração consiste em

Caso 1: $I=\emptyset$.

Devolva $L$ e pare.

Caso 2: $L=\emptyset$.

Seja $v$ o primeiro vértice na lista $I$.

Seja $I^{\prime}:=I \backslash\{v\}$.

Seja $L^{\prime}:=\langle v\rangle$.

Comece nova iteração com $L^{\prime}$ e $I^{\prime}$ nos papéis de $L$ e $I$. 
Caso 3: $I \neq \emptyset$ e $L \neq \emptyset$.

Seja $v$ o primeiro vértice na lista $I$.

Seja $I^{\prime}:=I \backslash\{v\}$.

Seja $u$ o pai de $v$ em $T$.

Caso 3A: Existe uma aresta de um descendente de $v$ em $T$ a um predecessor de $u$ em $L$. Seja $L^{\prime}$ a lista obtida após inserir $v$ imediatamente antes de $u$ em $L$.

Comece nova iteração com $L^{\prime}$ e $I^{\prime}$ nos papéis de $L$ e $I$.

Caso 3B: Existe uma aresta de um descendente de $v$ em $T$ a um sucessor de $u$ em $L$.

Seja $L^{\prime}$ a lista obtida após inserir $v$ imediatamente depois de $u$ em $L$.

Comece nova iteração com $L^{\prime}$ e $I^{\prime}$ nos papéis de $L$ e $I$.

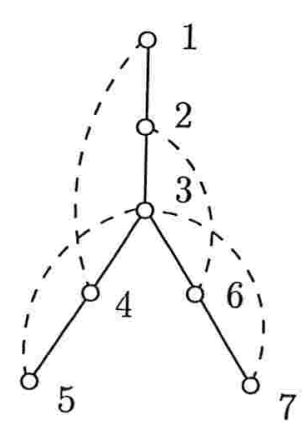

(a)

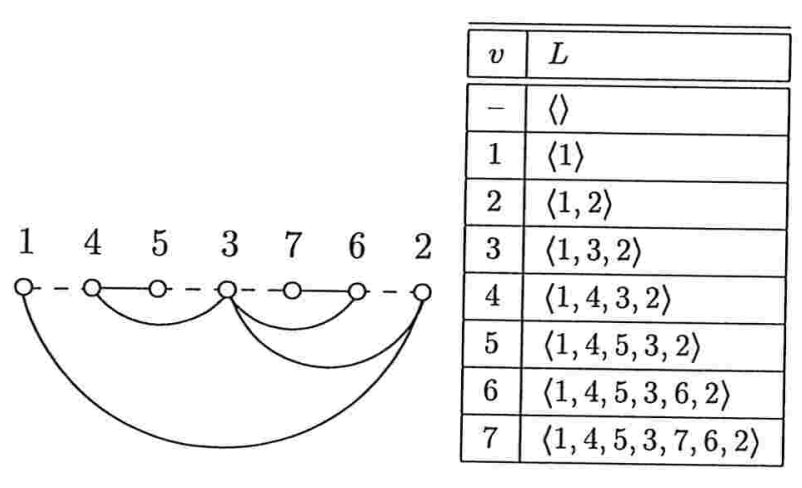

(b)

Figura 3.12: (a) As linhas sólidas ilustram uma bp-árvore de um grafo $G$ cujos vértices estão numerados de acordo com a visita pré-ordem na árvore. Inicialmente, $I=\langle 1,2,3,4,5,6,7\rangle$. (b) Uma lec-ordenação obtida pelo algoritmo lec-ordenação $(G)$ e a lista $L$ para cada vértice $v$ examinado pelo algoritmo.

\section{Busca por um $X Y$-caminho}

Seja $M$ uma moldura conexa e sejam $X$ e $Y$ subconjuntos de $V_{M}$. Para resolver o problema do $X Y$-caminho, seguimos os passos da demonstração do lema 3.3 . 
Encontrar um $X Y$-caminho ou uma $X Y$-obstrução numa moldura que é uma árvore não é uma tarefa difícil. O algoritmo a seguir resolve o problema do $X Y$-caminho manipulando uma árvore que representa a moldura dada.

Seja $H$ um grafo conexo e $\mathcal{B}$ o conjunto dos blocos de $H$. Pode-se definir de maneira natural um grafo bipartido que tem $V_{H} \cup \mathcal{B}$ como conjunto de vértices e arestas da forma $B v$ onde $B$ é um bloco de $H$ e $v$ é um vértice de $B$. É evidente que um tal grafo é uma árvore que denominamos de a árvore de blocos ${ }^{1}$ de $H$ (figura 3.13).

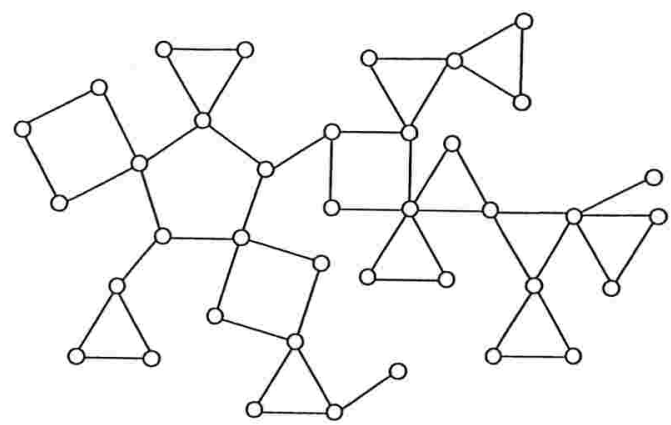

(a)

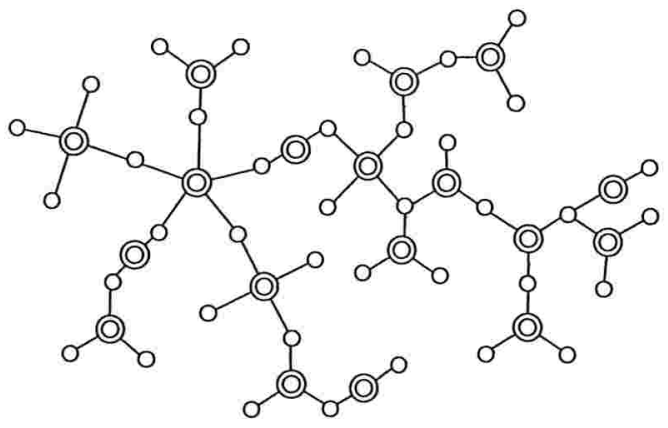

(b)

Figura 3.13: (a) Uma moldura e (b) sua respectiva árvore de blocos.

Seja $T$ a árvore de blocos de um grafo conexo $H$. Cada nó de $T$ que é um bloco de $H$ é dito um C-nó e um nó de $T$ que é um vértice de $H$ é chamado de P-nó.

Passamos agora a descrever o algoritmo que resolve o problema do $X Y$-caminho. $\mathrm{O}$ algoritmo utiliza a representação da moldura dada através de sua árvore de blocos ${ }^{2}$.

\begin{abstract}
Algoritmo $X Y$-caminho $(M, X, Y)$. Recebe uma moldura conexa $M$ e subconjuntos $X$ e $Y$ de $V_{M}$ e devolve um $X Y$-caminho ou uma $X Y$-obstrução em $M$.
\end{abstract}

Seja $T_{0}$ a árvore de blocos de $M$. O algoritmo é iterativo e cada iteração começa com uma subárvore $T$ de $T_{0}$, subconjuntos $X_{T}$ e $Y_{T}$ de $V_{T}$ e subconjuntos $W$ e $Z$ de $V_{M}$. Os conjuntos $X_{T}$ e $Y_{T}$ contém os nós da árvore $T$ que enxergam $X$ e $Y$ através de $E_{T_{0}} \backslash E_{T}$, respectivamente. Os conjuntos $W$ e $Z$ contém os P-nós da árvore $T_{0}$ que enxergam $X$ e $Y$ através de $E_{T_{0}} \backslash E_{T}$, respectivamente. No início da primeira iteração, $T=T_{0}, X_{T}=X, Y_{T}=Y, W=X$ e $Z=Y$. Cada iteração consiste em:

Caso 1: Toda folha de $T$ está em $X_{T} \cap Y_{T}$ e $T$ é um caminho.

\footnotetext{
${ }^{1}$ Por conveniência, esta definição é levemente diferente da usual [6].

${ }^{2} \mathrm{~A}$ estrutura da árvore de blocos de uma moldura assemelha-se à PC-árvore usada no próximo capítulo para representar e manipular molduras (seção 4.2).
} 
Seja $R$ o conjunto dos P-nós em $T$.

Para cada C-nó $C$ em $T$, seja $X_{C}:=V_{C} \cap(W \cup R)$ e $Y_{C}:=V_{C} \cap(Z \cup R)$.

Caso 1A: Cada C-nó $C$ em $T$ possui um caminho $P_{C}$ contendo $X_{C}$ e internamente disjunto de $Y_{C}$.

Seja $P_{T}$ o caminho em $M$ obtido através da concatenação dos caminhos em $\left\{P_{C}: C\right.$ é um C-nó em T $\}$.

Seja $P$ um caminho em $M$ contendo $P_{T}$ e com extremos em $X$ tal que para cada bloco $C$ na base de $P$, tem-se que $P$ contém os vértices em $V_{C} \cap W$.

Devolva $P$ e pare.

Caso 1B: Existe um C-nó $C$ em $T$ que não possui um caminho contendo $X_{C}$ e internamente disjunto de $Y_{C}$.

Sejam $v_{1}, v_{2}, v_{3}$ e $v_{4}$ vértices distintos de $C$ e que aparecem nesta ordem em $C$ tais que $v_{1}$ e $v_{3}$ estão em $X_{C}$ e $v_{2}$ e $v_{4}$ estão em $Y_{C}$.

Devolva $\left(C, v_{1}, v_{2}, v_{3}, v_{4}\right)$ e pare.

Caso 2: Toda folha de $T$ está em $X_{T} \cap Y_{T}$ e existe um nó $v$ em $T$ com grau maior que 2 .

Caso 2A: $v$ é um C-nó.

Seja $C$ o bloco de $M$ correspondente a $v$.

Sejam $v_{1}, v_{2}$ e $v_{3}$ P-nós distintos adjacentes a $v$ em $T$.

Devolva $\left(C, v_{1}, v_{2}, v_{3}\right)$ e pare.

Caso 2B: $v$ é um P-nó.

Sejam $C_{1}, C_{2}$ e $C_{3}$ C-nós distintos adjacentes a $v$ em $T$.

Sejam $K_{1}, K_{2}$ e $K_{3}$ componentes de $M-v$ tais que, para $i=1,2,3, C_{i}$ é bloco de $K_{i}+v$.

Devolva $\left(v, K_{1}, K_{2}, K_{3}\right)$ e pare.

Caso 3: Existe uma folha $f$ de $T$ que não está em $X_{T} \cap Y_{T}$.

Seja $u$ o nó de $T$ adjacente a $f$.

Seja $T^{\prime}:=T-f$.

Seja $X_{T^{\prime}}:=\left(X_{T} \backslash\{f\}\right) \cup\{u\}$ se $f$ está em $X_{T}$; caso contrário $X_{T^{\prime}}:=X_{T}$.

Seja $Y_{T^{\prime}}:=\left(Y_{T} \backslash\{f\}\right) \cup\{u\}$ se $f$ está em $Y_{T}$; caso contrário $Y_{T^{\prime \prime}}:=Y_{T}$.

Seja $W^{\prime}:=W \cup\{u\}$ se $f$ está em $X_{T}$ e $u$ é um P-nó; caso contrário $W^{\prime}:=W$.

Seja $Z^{\prime}:=Z \cup\{u\}$ se $f$ está em $Y_{T}$ e $u$ é um P-nó; caso contrário $Z^{\prime}:=Z$.

Comece nova iteração com $T^{\prime}, X_{T^{\prime}}, Y_{T^{\prime}}, W^{\prime}$ e $Z^{\prime}$ nos papéis de $T, X_{T}, Y_{T}, W$ e $Z$, respectivamente. 
A execução do algoritmo consiste de uma seqüência de "reduções" feitas no caso 3 seguida de uma ocorrência do caso 1 ou do caso 2 . O caso 3 do algoritmo realiza as reduções descritas nos fatos 3.4, 3.5 e 3.6 na demonstração do lema 3.3.

No caso $1 \mathrm{~A}$, o algoritmo pára após devolver um $X Y$-caminho $P$ em $M$. Já nos casos $1 \mathrm{~B}, 2 \mathrm{~A}$ e 2B, o algoritmo pára após devolver uma $X Y$-obstrução em $M$ : o caso $1 \mathrm{~B}$ devolve uma obstrução $\left(C, v_{1}, v_{2}, v_{3}, v_{4}\right)$ do tipo (o1); caso $2 \mathrm{~A}$ devolve uma obstrução $\left(C, v_{1}, v_{2}, v_{3}\right)$ do tipo (o2); e o caso $2 \mathrm{~B}$ devolve uma obstrução $\left(v, K_{1}, K_{2}, K_{3}\right)$ do tipo (o3).

Todos os blocos de um dado grafo podem ser determinados em tempo linear [8]. Dado um grafo e seus blocos, a árvore de blocos do grafo dado pode ser construída em tempo linear. Logo, a quantidade de tempo gasta pelo algoritmo com pré-processamento é proporcional a $\left|V_{M}\right|$.

Após uma ocorrência do caso 1 ou do caso 2, o algoritmo pára. O tempo gasto com esta ocorrência é proporcional a $\left|V_{M}\right|+\left|E_{M}\right|$. Como $\left|E_{M}\right|=O\left(\left|V_{M}\right|\right)$, então a quantidade total de tempo gasto com os casos 1 e 2 é $O\left(\left|V_{M}\right|\right)$.

O número de ocorrências do caso 3 é não superior a $\left|V_{M}\right|+\left|E_{M}\right|$. O tempo gasto com cada ocorrência do caso 3 é constante. Logo, o consumo total de tempo do algoritmo gasto com ocorrências do caso 3 é $O\left(\left|V_{M}\right|\right)$. Portanto, a quantidade total de tempo gasta pelo algoritmo é $O\left(\left|V_{M}\right|\right)$.

No início da última iteração, após a seqüência de reduções, as folhas de $T$ são chamadas de nós terminais. O conceito de nó terminal é usado de maneira fundamental pela implementação descrita no próximo capítulo.

\section{Construção da nova moldura}

Sejam $M$ uma moldura de um grafo conexo $H, X$ e $Y$ subconjuntos de $V_{M}, P$ um $X Y$ caminho em $M$ e um elemento $w$ que não está em $V_{H}$. O problema da construção da nova moldura é o mais simples dos três problemas. Na verdade, a sua solução está descrita em três linhas no caso 2B do algoritmo de LEC:

Seja $\bar{P}:=\left\langle w_{0}, w_{1}, \ldots, w_{k}\right\rangle$ o caminho complementar de $P$.

Seja $R$ o conjunto dos vértices em $V_{M} \backslash V_{\bar{P}}$ que não enxergam $Y \backslash V_{\bar{P}}$ através de $V_{M} \backslash V_{\bar{P}}$ (figura 3.8(b)).

Seja $M^{\prime}$ o grafo resultante após acrescentar-se o vértice $w$ e as arestas $w w_{0}$ e $w w_{k}$ ao grafo $M-R$ (figura $3.8(\mathrm{~d})$ ). 
Devido ao invariante (lec2) tem-se que o grafo $M^{\prime}$, na terceira linha acima, é uma moldura para o grafo $\left(V_{H} \cup\{w\}, E_{H} \cup\{w u: u \in X\}\right)$.

O caminho $\bar{P}$ pode ser construído em tempo $O\left(\left|V_{M}\right|\right)$. De fato, suponha que cada aresta de $M$ em $P$ foi marcada. Uma lista $\mathcal{L}$ com os blocos de $M$ pode ser determinada em tempo linear [8]. Os blocos na base $S$ de $P$ podem ser identificados percorrendo-se a lista $\mathcal{L}$ e examinando-se as arestas em cada bloco. A determinação de $S$ consome, portanto, tempo $O\left(\left|E_{S}\right|\right)=O\left(\left|V_{M}\right|\right)$. Finalmente, com $S$ e $P$ em mãos o caminho $\bar{P}$ pode ser obtido em tempo $O\left(\left|E_{S}\right|\right)$.

O conjunto $V_{M} \backslash R$ de vértices (e portanto $R$ ) pode ser construído através de uma busca em largura no grafo $M$ a partir dos vértices em $Y$. Na busca, os vértices em $\bar{P}$ não devem ser visitados. Esta busca em largura consome tempo $O\left(\left|V_{M}\right|\right)$.

Dados $R, w, w_{0}$ e $w_{k}$, o consumo de tempo para construir-se $M^{\prime}$ é proporcional a $\left|V_{M}\right|$.

Portanto, como o consumo de tempo de cada uma das três linhas acima do caso 2B é $O\left(\left|V_{M}\right|\right)$, então o problema da construção da nova moldura pode ser resolvido em tempo linear.

\section{Consumo de tempo do algoritmo de LEC}

O teorema a seguir mostra o consumo de tempo do algoritmo de LEC quando resolvemos os problemas da lec-ordenação, do $X Y$-caminho e da construção da nova moldura como descrito anteriormente.

Teorema 3.14 (da eficiência): O algoritmo de LEC gasta tempo $O\left(n^{2}+m^{2}\right)$.

Demonstração: Seja $G$ um grafo biconexo com $n$ vértices e $m$ arestas. O tempo gasto pelo algoritmo de LEC com o pré-processamento do grafo $G$ é dominado pelo consumo de tempo para obter-se uma lec-ordenação dos vértices de $G$. Isto pode ser feito em tempo $O\left(n^{2}+m^{2}\right)$.

Considere uma iteração em que ocorre o caso 2 do algoritmo de LEC. No início deste caso, o tempo gasto para determinar $w, L^{\prime}, X$ e $Y$ é linear. Decidir qual entre os casos $2 \mathrm{~A}$ e $2 \mathrm{~B}$ ocorre consiste em resolver o problema do $X Y$-caminho, o que pode ser feito em tempo proporcional a $\left|V_{M}\right|$. Uma ocorrência do caso $2 \mathrm{~A}$ gasta tempo constante. Já uma ocorrência do caso $2 \mathrm{~B}$ consiste, essencialmente, em construir uma nova moldura, o que pode ser feito em tempo linear. Como cada ocorrência do caso 2 gasta tempo $O(n+m)$ e este caso ocorre no máximo $n$ vezes, então o tempo total gasto com o caso 2 é $O(n(n+m))$.

Portanto, como o caso 1 ocorre no máximo uma vez e gasta tempo constante, então o consumo de tempo total do algoritmo é $O\left(n^{2}+m^{2}\right)$.

No próximo capítulo é apresentada uma descrição da implementação do algoritmo de LEC proposta por Shih e Hsu [15]. 


\section{Implementação de Shih e Hsu}

Neste capítulo é apresentada a implementação de Shih e Hsu [15] ("algoritmo de SH") para o algoritmo de LEC. Esta implementação resolve o problema da planaridade: dado um grafo $G$, ela devolve uma descrição combinatória plana de $G$ ou um subgrafo de Kuratowski. Neste capítulo é descrita apenas a parte da implementação referente ao teste de planaridade. Nos capítulos seguintes são tratados os aspectos da implementação referentes à determinação de um subgrafo de Kuratowski e à construção de uma descrição combinatória plana.

\subsection{Ordem de processamento dos vértices}

$\mathrm{Na}$ implementação de Shih e Hsu, os vértices são examinados de acordo com uma bp-ordenação $v_{1}, v_{2}, \ldots, v_{n}$ do grafo dado (capítulo 1 ). A cada iteração, a partir de uma moldura de $G\left[\left\{v_{1}, \ldots, v_{i-1}\right\}\right]$, o algoritmo, se possível, obtém uma moldura de $G\left[\left\{v_{1}, \ldots, v_{i-1}, v_{i}\right\}\right]$.

Uma bp-ordenação pode ser vista como uma "relaxação" da lec-ordenação. Seja $G$ um grafo conexo e $v_{1}, v_{2}, \ldots, v_{n}$ uma bp-ordenação de $G$ (em relação a uma bp-árvore arbitrária). Então o grafo

$$
G\left[\left\{v_{i}, \ldots, v_{n}\right\}\right] \text { é conexo para } i=1, \ldots, n .
$$

Como uma bp-ordenação não garante que $H:=G\left[\left\{v_{1}, \ldots, v_{i-1}\right\}\right]$ seja conexo, se existe uma moldura $M$ de $H$ e $H$ não é conexo, então $M$ também não é conexo. Com isso, para calcular uma moldura $M^{\prime}$ de $H^{\prime}:=H+v_{i}$, pode ser necessário calcular mais de um $X Y$-caminho em $M$.

\subsection{Estrutura de dados para a moldura}

O algoritmo de SH mantém uma moldura em $G$ do grafo $G\left[v_{1}, \ldots, v_{i-1}\right]$. A estrutura de dados usada, proposta por Shih e Hsu [15], é conhecida como PC-árvore e é definida a seguir. 


\section{PC-árvore}

Uma PC-árvore é uma árvore enraizada com dois tipos de nós - os P-nós e os C-nós - e com as seguintes propriedades:

(pc1) a raiz e as folhas são P-nós;

(pc2) os filhos de cada C-nó têm uma ordem.

Exceto pela raiz, cada nó tem um apontador para indicar quem é o seu pai na PC-árvore.

Considere o início da iteração do algoritmo de $\mathrm{SH}$ em que o vértice $v_{i}$ é examinado. Tal iteração começa com uma moldura $M$ do grafo $G\left[\left\{v_{1}, \ldots, v_{i-1}\right\}\right]$ dada por uma PC-árvore $T$ da seguinte maneira.

(T1) cada P-nó de $T$ é um vértice de $M$;

$B_{c} \quad$ (T2) cada C-nó $c$ de $T$ corresponde a um bloco $B_{c}$ de $M$ com pelo menos três arestas e vice-versa;

(T3) o pai de um C-nó $c$ é o vértice em $B_{c}$ de maior bp-número e os filhos de $c$ são os demais vértices de $B_{c}$;

(T4) cada C-nó $c$ vem acompanhado de uma lista, denotada por $\mathrm{RBC}(c)$ (Representative Boundary $(y c l e)^{1}$, com seu pai no início e seus filhos na ordem em que aparecem em $B_{c}$.

Veja as figuras 4.1(a) a (c). Diz-se que $T$ está associada à moldura $M$.

Note a semelhança entre tal PC-árvore e a árvore de blocos de $M$ (seção 3.7). Basicamente, além da orientação, a única diferença é que, na árvore de blocos, há nós representando blocos que consistem em apenas uma aresta, enquanto que na PC-árvore não.

É fácil manter uma PC-árvore se não há preocupação com o consumo de tempo do algoritmo. Para uma implementação quadrática, por exemplo, é trivial manter-se uma PC-árvore. Abaixo, segue uma descrição das modificações feitas na PC-árvore nesta implementação para garantir que o algoritmo gaste tempo linear no número de vértices do grafo dado. Uma dessas mudanças é em relação à atualização dos pais de alguns filhos dos C-nós que serão "englobados" por um novo C-nó. Essa mudança provoca a formação de caminhos em $T$ formados por C-nós consecutivos. Com uma idéia similar à heurística da compressão de caminhos da estrutura de dados conhecida como union-find [16], obtém-se algo melhor do que quadrático, porém possivelmente não-linear.

\footnotetext{
${ }^{1}$ Conforme é descrito mais adiante, a implementação usa uma PC-árvore alterada, onde é mantido uma versão "compacta" dessa lista.
} 


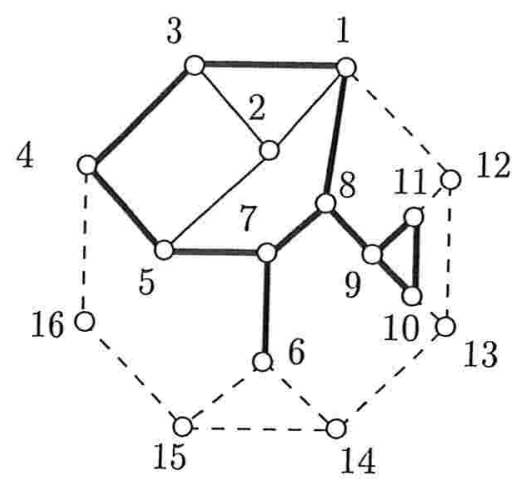

(a)

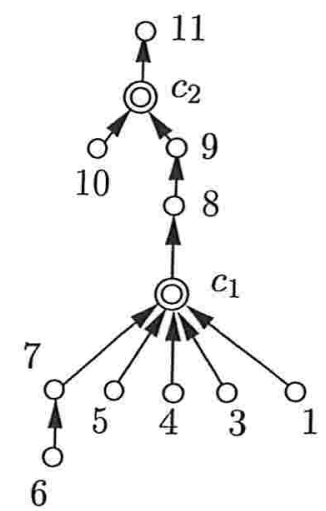

$$
\begin{aligned}
& R B C\left(c_{1}\right)=\langle 8,1,3,4,5,7\rangle \\
& R B C\left(c_{2}\right)=\langle 11,9,10\rangle
\end{aligned}
$$

(c)

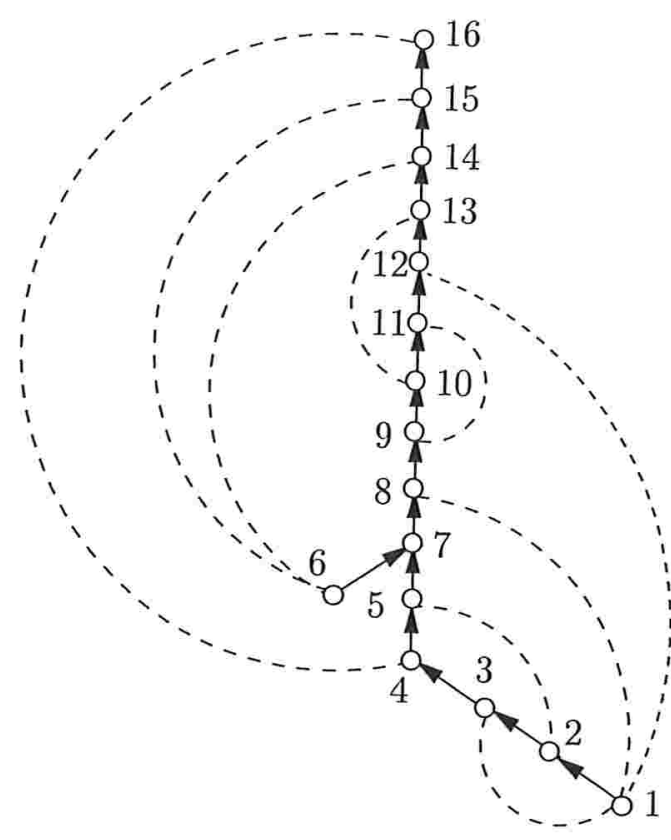

(b)

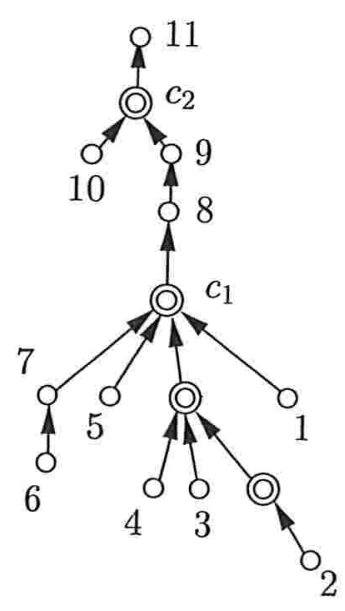

$R B C\left(c_{1}\right)=\langle 8,1,4,7\rangle$ $R B C\left(c_{2}\right)=\langle 11,9,10\rangle$

(d)

Figura 4.1: Os C-nós são ilustrados por dois círculos concêntricos e as setas indicam o pai de cada nó na PC-árvore. (a) Um grafo $G$ e uma moldura $M$ (em linhas grossas) de $G[\{1, \ldots, 11\}]$. (b) Uma bp-árvore de $G$. (c) Uma PC-árvore de $M$ e as listas $R B C\left(c_{1}\right)$ e $R B C\left(c_{2}\right)$. (d) Uma PC-árvore alterada, correspondente a (c). 


\section{PC-árvore alterada}

$\mathrm{Na}$ implementação aqui apresentada, para garantir que o tempo consumido pelo algoritmo seja linear, há duas diferenças fundamentais na maneira como a PC-árvore é mantida frente à descrição acima. A primeira diz respeito aos apontadores que indicam o pai dos filhos de um C-nó. A segunda, ao RBC de um C-nó. Essa maneira de implementar a PC-árvore do algoritmo de SH é chamada de PC-árvore alterada.

A primeira diferença, relativa aos filhos de um C-nó, grosseiramente falando, consiste na postergação da atualização de alguns apontadores para o pai. Como conseqüência de tal medida, permanecem na PC-árvore C-nós de blocos de iterações anteriores que não estão mais na moldura corrente. Tais C-nós são ditos inativos e são caracterizados por terem seu ponteiro pai apontando para um outro C-nó. Os C-nós que de fato correpondem a um bloco da moldura corrente são chamados de ativos e apenas C-nós ativos têm um RBC. Na verdade, além de C-nós inativos, ficam presentes na árvore, sem prejuízo para o algoritmo, vértices de $G$ que não fazem mais parte da moldura corrente. Veja na figura 4.1(d) um possível exemplo de PC-árvore alterada correspondente à PC-árvore exibida na figura 4.1(c). Note a presença do P-nó 2 na PC-árvore da figura $4.1(\mathrm{~d})$.

A segunda diferença consiste em manter-se uma versão "compacta" do RBC. Seja $L:=$ $L\left\{v_{i}, \ldots, v_{n}\right\}$. Para cada C-nó ativo $d$, vértices de $B_{d}$ que não enxergam $L$ através de $V_{G} \backslash V_{B_{d}}$ são omitidos do $\operatorname{RBC}(d)$ no momento em que são visitados pelo algoritmo. Por exemplo, os vértices 3 e 5 são omitidos do RBC do C-nó $c_{1}$ na figura 4.1(d).

Na PC-árvore alterada $T$, diz-se que um C-nó $c$ é o pai real de um P-nó $p$ (e que $p$ é o filho real de $c$ ) se o pai de $p$ é um C-nó e $c$ é o primeiro C-nó ativo encontrado no caminho de $p$ à raiz de $T$.

Abaixo são mostradas versões adaptadas de (T2), (T3) e (T4) à PC-árvore alterada:

(T2') cada C-nó ativo $c$ de $T$ corresponde a um bloco $B_{c}$ de $M$ com pelo menos três arestas e vice-versa;

(T3') cada C-nó inativo de $T$ é filho de um C-nó em $T$; o pai de um C-nó ativo $c$ é o vértice em $B_{c}$ de maior bp-número e os filhos reais de $c$ incluem os vértices de $B_{c}$ que enxergam $L$ através de $V_{G} \backslash V_{B_{c}}$;

(T4') cada C-nó ativo $c$ vem acompanhado de uma lista, denotada por $\mathrm{RBC}(c)$, com seu pai no início e os filhos reais de $c$ em $T$ que enxergam $L$ através de $V_{G} \backslash V_{B_{c}}$, na ordem em que aparecem em $B_{c}$.

Para que o percurso de uma PC-árvore alterada possa ser feito eficientemente, informações extras são necessárias. Cada P-nó que é filho de um C-nó tem um apontador para a célula 
do RBC correpondente a este P-nó, caso exista. Para cada C-nó ativo $d$, as células dos extremos do $\operatorname{RBC}(d)$ têm um apontador para $d$. Na seção 4.5, encontra-se uma descrição de como uma PC-árvore alterada é percorrida e como essas informações são utilizadas.

\section{Declaração de uma PC-árvore alterada}

A declaração de uma PC-árvore alterada $T$ na implementação do algoritmo de SH é ilustrada na figura 4.2. A variável parent/ $u$ ] guarda um apontador para o pai de $u$ em $T$. Para cada C-nó ativo $c$ de $T, R B C[c]$ é o $\operatorname{RBC}(c)$ armazenado numa lista do tipo bmlist.

Para cada P-nó $u$ cujo pai real é um C-nó ativo $c$, se $u$ está em $\mathrm{RBC}(c)$ então ptritem/u] aponta a célula correspondente a $u$ em $\operatorname{RBC}(c)$.

Para cada C-nó ativo $c$, sejam $i_{1}$ e $i_{2}$ apontadores para a primeira e a última célula de $\operatorname{RBC}(c)$, respectivamente. As variáveis activecnode $\left[i_{1}\right]$ e activecnode[i $\left.i_{2}\right]$ apontam para $c$.

Veja na figura 4.3 como estão as variáveis no caso da PC-árvore alterada da figura 4.1(d). $\langle$ declaração de uma PC-árvore 53$\rangle \equiv$

node_map $<$ node $>$ parent;

map $<$ node, bmlist $<$ node $>>R B C$;

map $<$ bmlist_item, node $>$ activecnode;

node_map $<$ bmlist_item $>$ ptritem;

Figura 4.2: Declaração de uma PC-árvore alterada na implementação do algoritmo de SH.

\subsection{Algoritmo de $\mathrm{SH}$}

Seja $G$ o grafo conexo dado. O algoritmo de SH constrói uma bp-árvore $T_{0}$ de $G$ e passa então à parte iterativa, onde os vértices de $G$ são examinados um após o outro, de acordo com uma bp-ordenação em relação a $T_{0}$.

Cada iteração começa com o conjunto $L$ dos vértices não-examinados e uma árvore enraizada $T$ tal que $T[L]=T_{0}[L]$ e $T\left[V_{G} \backslash L\right]$ é uma PC-árvore alterada associada a uma moldura $M$ em $G$ do grafo $G\left[V_{G} \backslash L\right]$. (No algoritmo de SH, $G\left[V_{G} \backslash L\right]$ e $M$ podem não ser conexos. Algumas vezes, diz-se, para simplificar, que $T$ é a PC-árvore.) Inicialmente, $L=V_{G}$ e $T=T_{0}$. Seja $w$ o vértice em $L$ com o menor bp-número; $w$ é o vértice examinado nesta iteração.

Sejam $X:=\left\{u \in V_{M}: u w \in E_{G}\right\}$ e $Y:=\left\{u \in V_{M}\right.$ : existe $\left.v \in V_{G} \backslash\left(V_{H} \cup\{w\}\right), u v \in E_{G}\right\}$. Em cada iteração do algoritmo de $\mathrm{SH}$, busca-se uma $X Y$-obstrução. A primeira etapa nessa $X, Y$ busca é a determinação dos chamados terminais de $T$ (definidos na seção 4.6), que indicarão os 

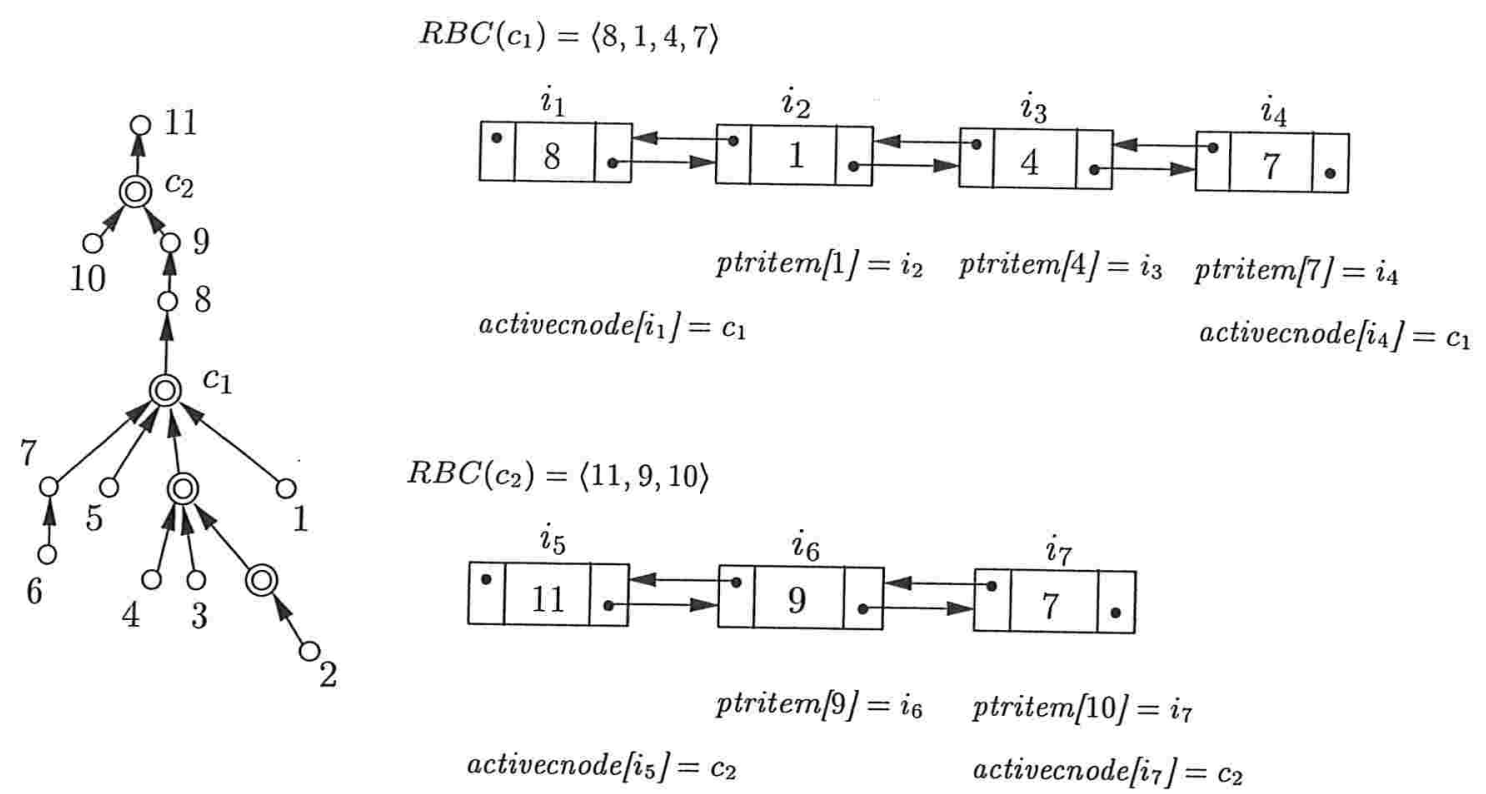

Figura 4.3: Estrutura de dados de uma PC-árvore alterada.

candidatos a ponta dos $X Y$-caminhos em $M$. Se, na determinação de algum dos $X Y$-caminhos, uma $X Y$-obstrução é detectada, o algoritmo pára. Caso contrário, a PC-árvore $T$ é atualizada de forma a incluir os novos blocos e vai-se para a próxima iteração. Isto é feito pela função is_planar (figura 4.4), que é descrita a seguir.

As inicializações são realizadas pela função pre_processing, que constrói uma bp-árvore $T_{0}$ de $G$ e, para cada vértice de $G$, atribui um bp-número (variável dfspos_num/ /). Além disso, essa função calcula outras informações importantes para o algoritmo de $\overline{\mathrm{SH}}$, que são mencionadas mais adiante, conforme se tornam necessárias.

Na próxima seção, encontra-se uma descrição das informações associadas a cada vértice de $G$ (valores $b$ e $h$ ) que são utilizadas para calcular os terminais e $X Y$-caminhos ou $X Y$-obstruções.

\subsection{Valores de $b$ para cada nó}

Durante a execução do algoritmo de $\mathrm{SH}$, cada nó $u$ na PC-árvore corrente $T$ mantém o bp-número do vértice mais "alto" em $T_{0}$ que é vizinho, no grafo $G$, de um descendente de $u$ em $T$. $h(u)$ Mais precisamente, seja $h(u)$ o maior bp-número (na bp-árvore $T_{0}$ ) de um vizinho de $u$ em $G$. $b(u)$ Durante a execução, o algoritmo mantém, para cada nó $u$ de $T$, um número $b(u)$, que é o maior valor de $h(v)$ tal que $v$ é um descendente de $u$ na PC-árvore corrente $T$ (figura 4.5).

$\mathrm{Na}$ implementação descrita aqui, os valores de $h$ e de $b$ são representados pelas variáveis 
55 〈função principal testar planaridade 55) 三

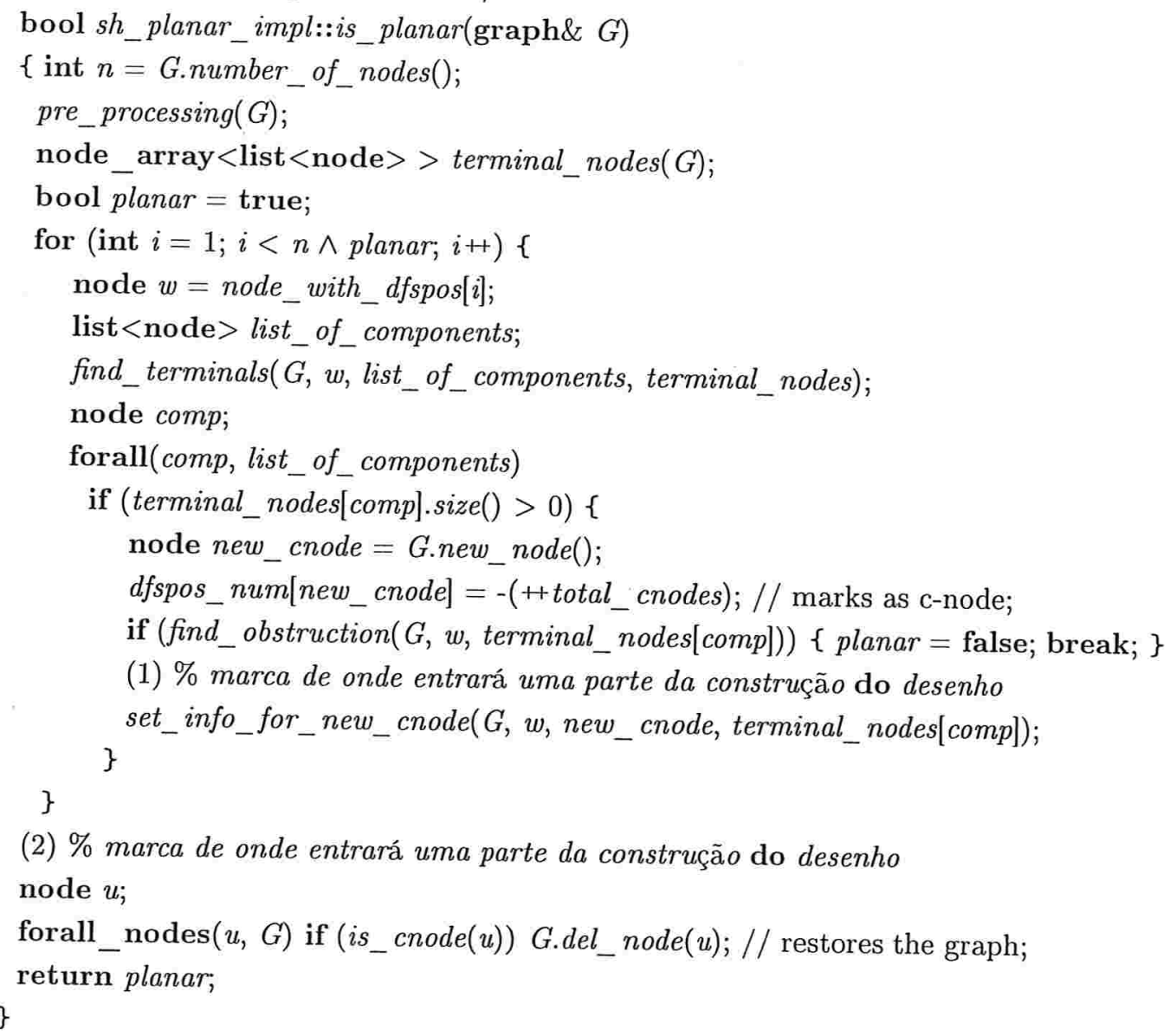

Figura 4.4: Código da função is_planar. 
largest_neighbor [ ] e label_b / ], respectivamente. O cálculo de $h$ é feito somente uma vez, durante o pré-processamento do algoritmo, pela função pre_processing, onde também é calculado o valor inicial de $b$ de cada vértice.

Os valores de $b$ e de $h$ são extremamente utilizados durante o cálculo dos terminais, na seção 4.6, e na determinação dos $X Y$-caminhos ou deteç̧ão de uma $X Y$-obstrução, na seção 4.7. Por exemplo, seja $c$ um C-nó ativo em $T$ e seja $u$ um vértice em $\operatorname{RBC}(c)$. Para determinar se $u$ enxerga $L \backslash\{w\}$ através de $V_{G} \backslash V_{B_{c}}$, basta verificar se $b(u)>\operatorname{bp}(w)$, sendo $w$ o primeiro elemento em $L$.

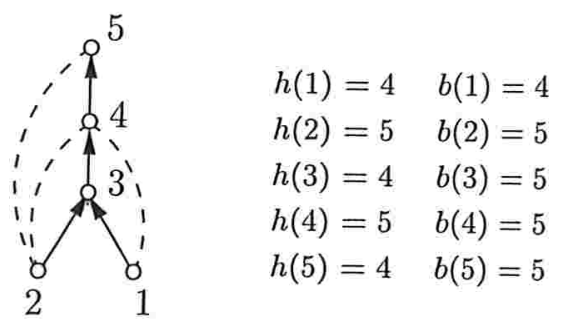

(a)

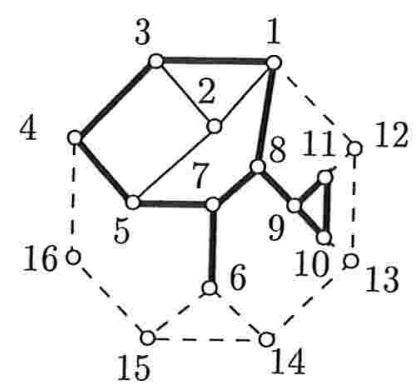

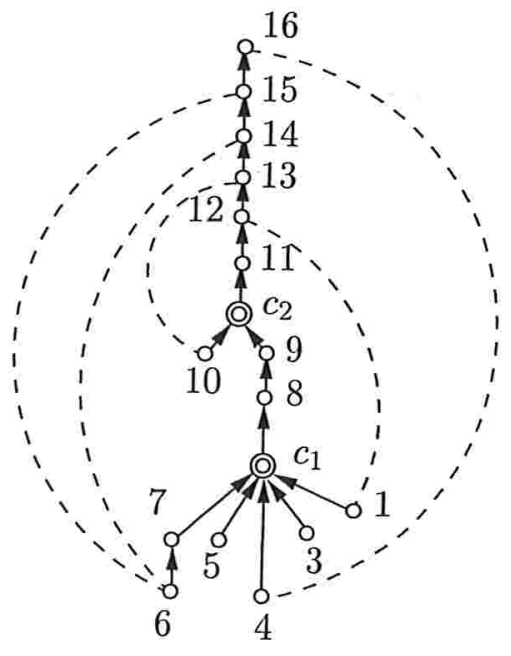

(b)

$$
\begin{array}{ll}
h(1)=12 & b(1)=12 \\
h(3)=4 & b(3)=4 \\
h(4)=16 & b(4)=16 \\
h(5)=7 & b(5)=7 \\
h(6)=15 & b(6)=15 \\
h(7)=8 & b(7)=15 \\
& b\left(c_{1}\right)=16 \\
h(8)=9 & b(8)=16 \\
h(9)=11 & b(9)=16 \\
h(10)=13 & b(10)=13 \\
& b\left(c_{2}\right)=16 \\
h(11)=12 & b(11)=16 \\
h(12)=13 & b(12)=16 \\
h(13)=14 & b(13)=16 \\
h(14)=15 & b(14)=16 \\
h(15)=16 & b(15)=16 \\
h(16)=15 & b(16)=16
\end{array}
$$

Figura 4.5: (a) Uma bp-árvore de um grafo juntamente com as demais arestas e os valores de $h$ e $b$ para cada nó. (b) Um grafo $G$ e uma moldura $M$ de $G[\{1, \ldots, 11\}]$ (em linhas grossas), uma PC-árvore correspondente a $M$ e os valores de $h$ e de $b$ para essa PC-árvore. 


\subsection{Percurso numa PC-árvore alterada}

Antes de passar à determinação dos terminais, vale a pena explicar como é feito o percurso numa PC-árvore alterada.

Para garantir a linearidade do algoritmo, a PC-árvore alterada é percorrida da seguinte maneira. A partir de um P-nó, sobe-se normalmente na PC-árvore por meio dos apontadores pai, exceto quando chega-se a um P-nó $u$ que é filho de um C-nó. Neste ponto, a função find_active_cnode é acionada (figura 4.6), com o P-nó $u$ como parâmetro. Essa função devolve um apontador para o C-nó ativo que é o pai real de $u$. Seja $c$ o pai real de $u$. Antes da primeira execução dessa função, cada vértice em $\mathrm{RBC}(c)$ tem a marca NOT_VISITED na variável state[ ]. Após uma execução dessa função, cada vértice percorrido neste processo está marcado como VISITED _ IN _ RBC e para cada um desses vértices, exceto pelo pai de $c$, seu ponteiro pai passa a apontar para $c$.

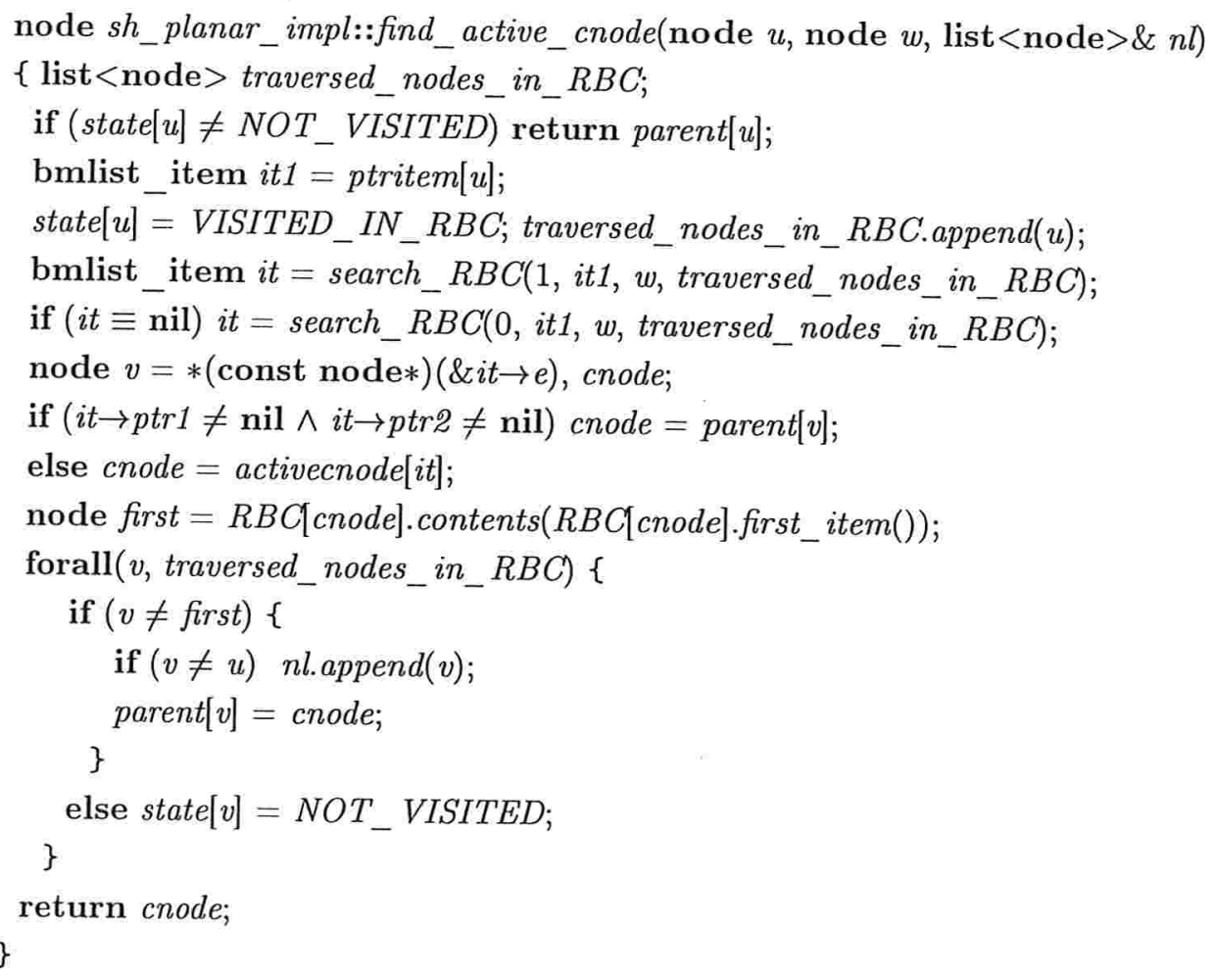

Figura 4.6: Código da função find_active_cnode.

A busca do pai real do P-nó $u$ é feita da seguinte maneira. Parte-se da célula de $u$ indicada pelo apontador ptritem[u] (variável it1 na função find_active_cnode), e percorre-se o RBC em questão numa direção arbitrária, até atingir: 
(c1) ou uma das extremidades do RBC;

(c2) ou uma célula que corresponde a um vértice marcado como VISITED _ IN_RBC;

(c3) ou uma célula que corresponde a um vértice que enxerga $L$ através de $V_{G} \backslash V_{B_{c}}$.

Seja $q$ a célula atingida. Se $q$ satisfaz (c1) ou (c2), então o C-nó ativo é indicado ou pelo apontador activecnode [q], ou pelo seu apontador pai, respectivamente. Caso contrário, a rotina percorre o RBC a partir de $u$, agora na outra direção, como descrito anteriormente, exceto ignorando a condição (c3). Observe que neste segundo percurso, se ocorrer a condição (c3) então uma obstrução do tipo (o1) é detectada. Este fato é ignorado neste ponto e é tratado somente durante a busca de um $X Y$-caminho (seção 4.7). Isto é feito desta maneira devido a parte do algoritmo que encontra um subgrafo de Kuratowski no grafo, descrita no capítulo 6.

O percurso do RBC numa dada direção é feito pela função search_RBC (figura 4.7) e uma ilustração do processo de determinação de um C-nó ativo se encontra na figura 4.8.

〈função auxiliar para procurar c-nó ativo 58) 三

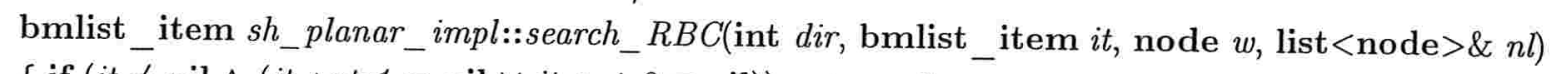

$\{$ if $(i t \overline{\neq} \operatorname{nil} \wedge(i t \rightarrow p t r 1 \equiv$ nil $\vee i t \rightarrow p t r 2 \equiv$ nil $))$ return $i t$

bmlist_item prev $=i t$, aux1 $=i t \rightarrow p t r 1$, aux $2=i t \rightarrow p t r 2$;

if (dir $\equiv 1)$ leda_swap(aux1, aux2);

it $=$ aux 1 ;

node $u=*($ const node $*)(\& i t \rightarrow e)$;

int $b=$ label_ $b[u]$;

while $\left(i t \neq \operatorname{nil} \wedge\left(b \leq d f s p o s_{-} n u m[w] \vee \operatorname{dir} \neq 1\right) \wedge\right.$ state $[u] \equiv N O T_{-}$VISITED $)\{$

aux1 $=i t \rightarrow p \operatorname{tr} 1$;

if (aux1 $\equiv$ prev) aux1 = it $\rightarrow$ ptr2;

prev $=i t ;$ it $=$ aux 1 ;

$u=*($ const node $*)(\& p r e v \rightarrow e)$;

state $[u]=V I S I T E D_{-} I N_{-} R B C ;$ nl.append $(u)$;

if $(i t \neq$ nil $)\{u=*($ const node $*)(\& i t \rightarrow e) ; b=$ label_b $[u] ;\}$

\}

if $($ it $\equiv$ nil) return prev;

else if $\left(\right.$ state $[u] \neq N O T_{-} V I S I T E D \vee(i t \rightarrow p t r 1 \equiv$ nil $\vee i t \rightarrow p t r 2 \equiv$ nil $\left.)\right)$ return $i t$; else return nil;

\}

Figura 4.7: Código da função search_RBC.

Para simplificar a exposição e o entendimento dos próximos procedimentos, deste ponto até a análise do algoritmo, que é feita na seção 4.9, a PC-árvore alterada é muitas vezes confundida 


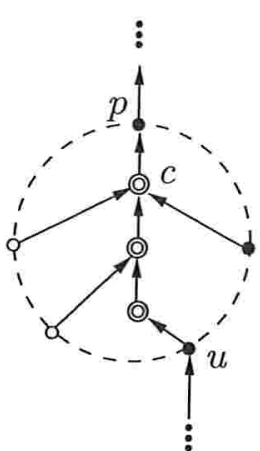

(a)

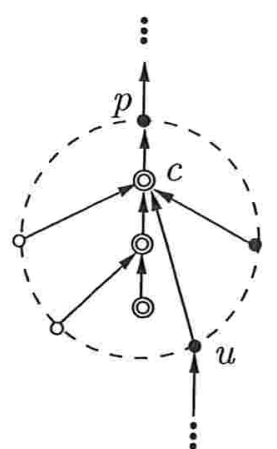

(b)

Figura 4.8: Cálculo do C-nó ativo $c$ a partir de um P-nó $u$. O $\mathrm{RBC}(c)$ é ilustrado por linhas tracejadas e $p$ é o pai de $c$. (a) Os vértices do $\operatorname{RBC}(c)$ que foram percorridos durante o processo estão ilustrados por círculos pretos. (b) Cada vértice percorrido, exceto $p$, tem seu pai atualizado, apontando para $c$.

com a PC-árvore original e fica implícito que a implementação está lidando, na verdade, com uma PC-árvore alterada.

\subsection{Determinação dos terminais}

As arestas de $G$ que não estão em $T_{0}$ são chamadas de arestas de retorno. (Na figura 4.5, as arestas de retorno são ilustradas por linhas tracejadas.) Seja $R$ o grafo obtido de $T$ pela inclusão das arestas de retorno com uma ponta em $L$ e outra em $V_{G} \backslash L$ (PC-árvore da figura 4.5(b)), e seja $L^{\prime}:=L \backslash\{w\}$. Um nó $t$ de $T-L$ é um terminal se, em $R$,

(nt1) $t$ enxerga $\{w\}$ e $L^{\prime}$ através de $V_{T_{t}}$ (onde $T_{t}$ é a subárvore de $T$ enraizada em $t$ );

(nt2) nenhum descendente próprio $t^{\prime}$ de $t$ em $T$ enxerga $\{w\}$ e $L^{\prime}$ através de $V_{T_{t^{\prime}}}$.

Na figura 4.5(b), para $L=\{12, \ldots, 16\}$ e $w=12$, o C-nó $c_{1}$ é terminal.

Os valores de $b$, definidos para cada nó em $T$, podem ser usados na determinação dos terminais, como mostra o seguinte lema.

Lema 4.1: Um terminal de $T-L$ é um nó $t$ que satisfaz as seguintes propriedades:

(t1) $b(t)>\operatorname{bp}(w)$;

(t2) $t$ é vizinho de $w$ em $G$ ou $t$ tem um descendente próprio em $T$ que é vizinho de $w$ em $G$; 
(t3) nenhum descendente próprio de $t$ satisfaz as propriedades ( $t 1)$ e (t2) simultaneamente.

Demonstração: A seguir, é demonstrado que vale (nt1) se e somente se valem (t1) e (t2).

Seja $t$ um nó de $T-L$ que satisfaz (t1). Então $t$ tem um descendente $u$ em $T$ tal que $h(u)>\operatorname{bp}(w)$, ou seja, $u$ é vizinho de um vértice em $L^{\prime}$ e portanto $t$ enxerga $L^{\prime}$ através de $V_{T_{t}}$. Se $t$ satisfaz (t2), então $t$ tem um descendente $u$ em $T$ tal que $h(u)=\mathrm{bp}(w)$, ou seja, $u$ é vizinho de $w$ e portanto $t$ enxerga $\{w\}$ através de $V_{T_{t}}$. Portanto, dadas as condições (t1) e (t2), a condição (nt1) da definição de nó terminal é satisfeita.

Agora suponha que $t$ satisfaz a condição (nt1). Se $t$ enxerga $L^{\prime}$ através de $V_{T_{t}}$, então $t$ tem um descendente $u$ em $T$ tal que $h(u)>\mathrm{bp}(w)$, ou seja, $b(t)>\operatorname{bp}(w)$. Se $t$ enxerga $\{w\}$ através de $V_{T_{t}}$, então $t$ é vizinho de $w$ em $G$ ou $t$ tem um descendente em $T$ que é vizinho de $w$ em $G$. Portanto, dada a condição (nt1), as condições (t1) e (t2) são satisfeitas.

Claramente as condições (nt2) e (t3) são equivalentes.

Na figura 4.5(b), em que $c_{1}$ é um terminal para $L=\{12, \ldots, 16\}$ e $w=12$, veja que $b\left(c_{1}\right)=$ $16>12=\operatorname{bp}(w)$ (condição (t1) do lema 4.1), 1 é vizinho de $w$ e é filho de $c_{1}$ (condição (t2)), e $c_{1}$ satisfaz (t3).

Observe que, se $L=\{w\}$ num dado momento da execução do algoritmo, então $w$ é a raiz da PC-árvore corrente $T$ e tem-se que, para cada nó $t$ de $T$, vale que $b(t) \leq \mathrm{bp}(w)$. Neste caso, não existe terminal em $T-L$ e o grafo em questão é planar. Observe também que se, num dado momento do algoritmo, se não há terminais em $T-L$, então não existe $X Y$-obstrução na moldura corrente e o algoritmo passa para a próxima iteração.

O lema 4.1 sugere o seguinte processo para determinar os terminais de $T-L$. Dado $w$ tal que $w$ não é a raiz de $T$, para cada vizinho $u$ de $w$ que é descendente de $w$ em $T$ (ou seja, tal que $\mathrm{bp}(u)<\mathrm{bp}(w))$, percorra $T$ a partir de $u$, subindo em direção a $w$ em $T$, até o primeiro nó $v$ tal que $b(v)>\operatorname{bp}(w)$, caso exista, marcando $v$ como terminal.

Infelizmente, um nó $v$ marcado por esse processo pode não ser um terminal pois esse processo pode ter marcado também um descendente próprio de $v$. Na figura $4.5(\mathrm{a})$, considere $w=4$. Partindo de 1, o processo marca 3 como um terminal. Partindo de 2, o processo marca 2 como terminal. Por causa de 2, o nó 3 não é um terminal. Além disso, pelo processo acima, um vértice pode ser desnecessariamente examinado várias vezes, conforme ilustrado na figura 4.9. Para $w=v_{1}, i=2, \ldots, j, v_{i}$ é examinado $k$ vezes no processo de encontrar os terminais, onde $v_{2}$ é o único terminal.

Para consertar o processo e torná-lo mais rápido, marca-se todos os nós de $T-L$ inicialmente como não-visitados, marca-se $w$ como visitado e percorre-se $T$ a partir de $u$ em direção a $w$, 


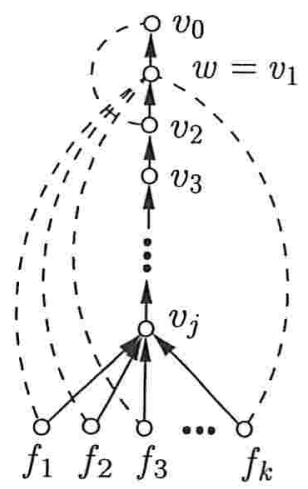

Figura 4.9: As setas indicam o pai de cada nó da PC-árvore ilustrada.

parando quando atingir-se um nó $v$ tal que

(1) $v$ já foi visitado ou

(2) $b(v)>\operatorname{bp}(w)$.

No caso (1), não há nada a fazer além de marcar os nós percorridos como visitados. No caso (2), inclui-se $v$ numa lista de candidatos a terminais, marca-se em $v$ a posição de $v$ nessa lista e prossegue-se subindo até um nó $v^{\prime}$ já visitado. Ao chegar-se em $v^{\prime}$, remove-se $v^{\prime}$ da lista de candidatos a terminais se ele estiver lá. Além disso, marca-se todos os nós percorridos como visitados. Ao final desse processo, a lista de candidatos a terminais é exatamente a lista de terminais dessa iteração.

O cálculo dos terminais é feito pela função find_terminals (figura 4.10), que é descrita a seguir.

A função find_terminals recebe como entrada o grafo $G$ e o vértice $w$, que é o vértice correntemente examinado pelo algoritmo de $\mathrm{SH}$, além da PC-árvore $T$ da iteração corrente (variáveis globais parent [ ], $R B C[$ ], ptritem e activecnode).

O parâmetro de saída list_of_components da função find_terminals é a lista das raízes das árvores de $T-L$ que contém algum terminal. O parâmetro de saída terminal_nodes [ ] guarda a lista dos terminais (e candidatos a terminais durante o processo) de cada componente de $T-L$.

A variável global statel / contém a marca VISITED, TERMINAL, NOT_VISITED ou VISITED _ IN_RBC para cada nó. A marca VISITED_IN_RBC é usada na determinação dos C-nós ativos, conforme descrita na seção 4.5. Na fase inicial do algoritmo de SH, state[u] é inicializado com NOT_VISITED para cada nó $u$. No final da função find_terminals, para cada nó $u$ que teve seu estado alterado, state $[u]$ é restaurado para NOT_VISITED. Durante o processo, a marca TERMINAL indica os candidatos a terminais. 
$\langle$ cálculo de terminais 62$\rangle \equiv$

void $s h \_p l a n a r_{-} i m p l:: f_{i n d}$ terminals(graph\& $G$, node $w$, list $<$ node $>\&$ list_of_components,

$\{$ map $<$ node, list_item $>$ terminal_nodes_item; node_array $<$ list $<$ node $>>\&$ terminal_nodes $)$

map $<$ node, node $>$ component_of;

list $<$ node $>$ traversed_nodes;

component_of $[w]=$ nil; state $[w]=V I S I T E D ;$ traversed_nodes.append $(w) ;$

edge $e$;

forall_out_edges $(e, w)\{$

node $u=\operatorname{target}(e)$;

if $\left(d f s p o s \_n u m[u]<d f s p o s \_n u m[w] \wedge\right.$ is_back_edge $\left.(e)\right)\{$

list $<$ node $>S$;

node terminal_node $=$ nil;

(1) while (state $\left.[u] \equiv N O T_{\text {_VISITED }}\right)\{$

if (is_cnode (parent $[u]))\{$ \} node cnode $=$ find_active_cnode $(u, w$, traversed_nodes $) ;$ counter $[$ cnode $]++$;

if $\left(\right.$ terminal_node $\equiv$ nil $\wedge$ label_b $\left.[u]>d f s p o s \_n u m[w]\right)\{$ state $[u]=T E R M I N A L ;$ terminal_node $=u$; \}

else state $[u]=$ VISITED;

traversed_nodes.append $(u) ; \operatorname{S.push}(u) ; u=\operatorname{parent}[u]$;

\} // end while;

if $(u \equiv w)\{$ \}

node $c=S . p o p() ;$ component_of $[c]=c$; list_of_components.append $(c)$;

(2) while ( $\neg$ S.empty ()$)\{$

node $u=S . p o p() ;$ component_of $u]=$ component_offparent $[u]]$; \}

if $($ terminal_node $\neq$ nil $)\{$

node $c=$ component_ofterminal_node];

terminal_nodes_item $[$ terminal_node $]=$ terminal_nodes $[c] \cdot$ append $($ terminal_node $)$;

if (state $[u] \equiv T E R M I N A L)\}$ \}

state $[u]=V I S I T E D ;$ terminal_nodes $[c] \cdot d e l($ terminal_nodes_item $[u]) ;$

\}

\} // end if (dfspos_num[u] $<$ dfspos_num[w] \&\& is_back_edge(e));

\} // end forall_out_edges;

node $u$;

forall ( $u$, traversed_nodes) state $[u]=N O T_{-}$VISITED;

\}

Figura 4.10: Código da função find_terminals. 
Para cada candidato $v$ a terminal, a variável terminal_nodes_item/v/ guarda a posição de $v$ na lista terminal_nodes [ ] e, para cada C-nó ativo $d$, counter[d] contém o número de filhos reais de $d$ que são visitados por esse processo (é o número de filhos reais de $d$ que têm um descendente em $T$ que é vizinho de $w$ em $G$ ). Para cada vértice visitado $u$, guarda-se na variável auxiliar component_of[u] a raiz da árvore de $T-L$ que contém $u$. A variável $S$ é uma variável auxiliar que guarda os vértices visitados até determinar-se o componente em que estão.

Após a construção da lista de terminais para cada componente de $T-L$ pela função acima, uma iteração do algoritmo de LEC é aplicada para cada componente com pelo menos um terminal.

\subsection{Busca por uma $X Y$-obstrução}

Seja $M$ uma moldura em $G$ de $H:=G\left[V_{G} \backslash L\right]$ dada por uma PC-árvore $T$. Seja $w$ o vértice de menor bp-número em $L$. Sejam $X:=\left\{u \in V_{M}: u w \in E_{G}\right\}$ e $Y:=\{u \in$ $V_{M}$ : existe $\left.v \in V_{G} \backslash\left(V_{H} \cup\{w\}\right), u v \in E_{G}\right\}$. O algoritmo de SH verifica a presença de uma $X Y$-obstrução em cada componente de $T\left[V_{G} \backslash L\right]$ com terminais. Isto é feito pela função find_obstruction e seu código é ilustrado nas figuras 4.11 e 4.12 .

bool $s h_{-}$planar_impl:: find_obstruction(graph\& $G$, node $w$, list $<$ node $>\&$ terminal_nodes)

$\{$ cnode_of_possible_k33_obstruction $=$ nil; // reset global variable;

list $<$ node $>$ list_terminal $=$ terminal_nodes;

switch(terminal_nodes.size()) \{

case 1:

$\{$ node $t 1=$ list_terminal.pop () ;

if (test_obstruction_from_terminal $(G, w, t 1, w))$ return true;

if (cnode_of_possible_k33_obstruction $\neq$ nil)

$\left\{\right.$ node $v=f i n d \_n o d e \_w i t h \_l a b e l_{-} b_{-} g r e a t e r \_t h a n \_d f s_{-} w($

false, $G$, cnode_of_possible_k33_obstruction, w);

if $(v \neq$ nil $)\{$

(2) \% marca de onde entrará a construção da lista de arcos da obstrução return true;

\}

\}

\}

break;

Figura 4.11: Código da função find_obstruction.

Existem três casos a serem considerados: o caso em que há um único terminal, o caso em que 
case 2:

$\{$ node $t 1=$ list_terminal.pop ()$, t 2=$ list_terminal.pop (), $m=l c a \_b e t w e e n \_t e r m \_n o d e s(t 1, t 2)$;

list_current_terminal_nodes.append(t1); list_current_terminal_nodes.append(t2);

node $t 12=$ last_pnode $(t 1, m), t 22=$ last_pnode $(t 2, m)$;

if (test_obstruction_from_terminal $(G, w, t 1, t 12))$ return true;

if (cnode_of_possible_k33_obstruction $\neq$ nil) \{

(2) \% marca de onde entrará a construção da lista de arcos da obstrução return true;

\}

if (test_obstruction_from_terminal $(G, w, t 2, t 22))$ return true;

if (cnode_of_possible_k33_obstruction $\neq$ nil) \{

(2) \% marca de onde entrará a construção da lista de arcos da obstrução return true;

\}

if $($ is_cnode $(m) \wedge$ test_cnode_counter $(G, m, w, t 12, t 22))\{$

(1) \% marca de onde entrará a construção da lista de arcos da obstrução return true;

\}

node $v=f i n d_{-}$node_with_label_b_greater_than_dfs_w(true, $\left.G, m, w\right)$; if $(v \neq \mathbf{n i l})\{$

(3) \% marca de onde entrará a construção da lista de arcos da obstrução return true;

\}

\}

break;

default:

\{

(4) \% marca de onde entrará a construção da lista de arcos da obstrução return true;

\}

\} // end switch;

return false;

\}

Figura 4.12: (Continuação) Código da função find_obstruction. 
há dois terminais e o caso em que há três ou mais terminais no componente.

$\mathrm{O}$ caso em que há um terminal, digamos $t$, é tratado da seguinte forma. Seja $Q$ o caminho de $t$ a $w$ em $T$. A função test_obstruction_from_terminal (figura 4.13) examina cada C-nó ativo em $Q$, subindo em $T$ e pára quando ocorrer uma das situações ilustradas nas figuras 4.14(a) e (b). Caso seja detectada a situação da figura 4.14(a), uma obstrução do tipo (o1) foi encontrada. Caso contrário, se existe um C-nó ativo em $Q$ nas condições da figura 4.14(b), a variável global cnode_of_possible_k33_obstruction passa a apontar para o primeiro C-nó detectado nessas condições. Seja $d$ tal C-nó e seja $Q^{\prime}$ o subcaminho de $Q$ do pai de $d$ até o filho de $w$ em $Q$. Neste caso, é preciso fazer o seguinte teste do caminho $Q^{\prime}$.

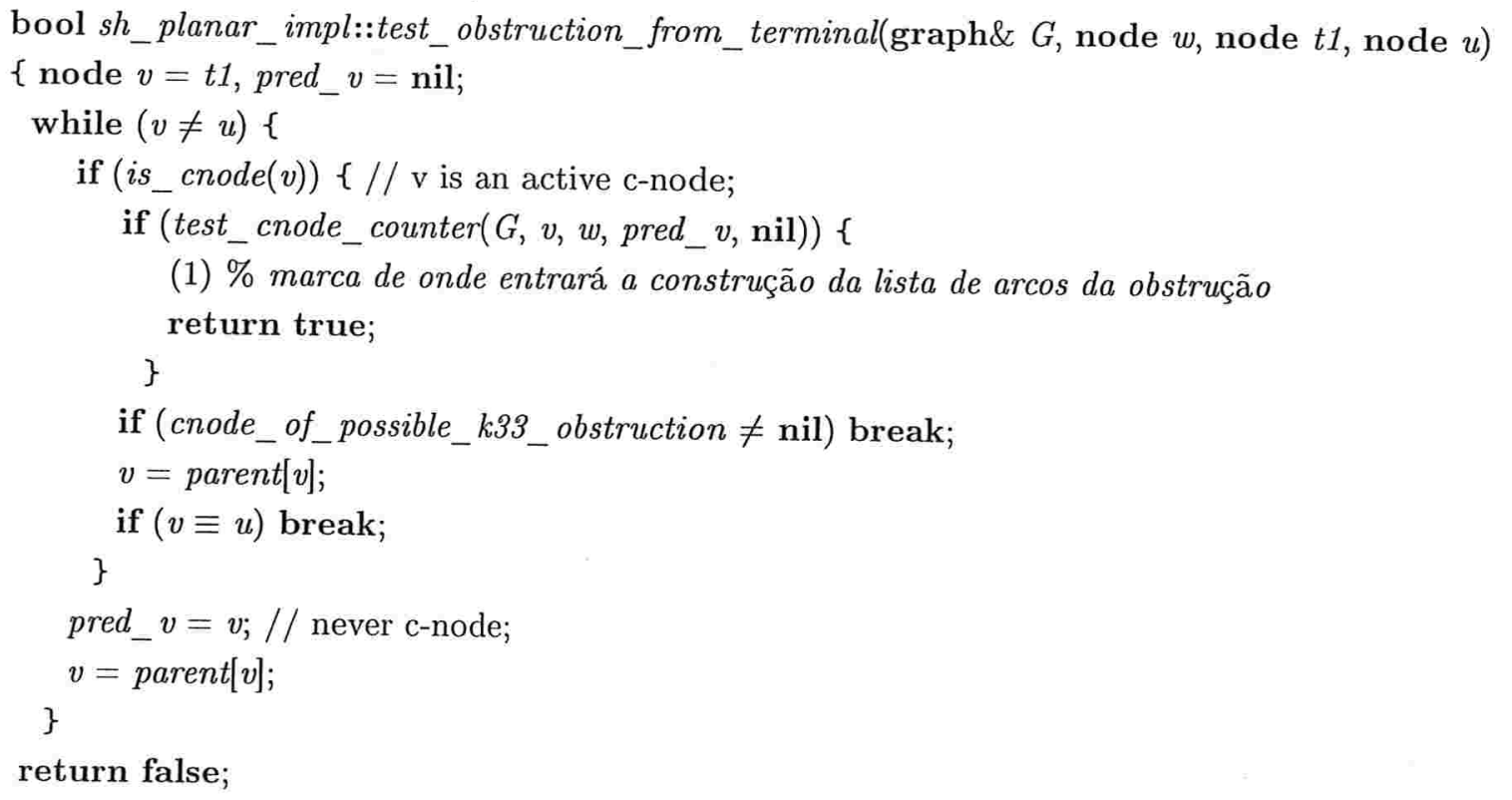

Figura 4.13: Código da função test_obstruction_from_terminal.

Seja $v$ um nó em $Q^{\prime}$ e seja $T_{v}^{*}:=T_{v}-T_{v^{\prime}}$, onde $v^{\prime}$ é o filho de $v$ em $Q$. É preciso verificar se existe um nó $v$ em $Q^{\prime}$ tal que $T_{v}^{*}$ contém um vizinho de um vértice em $L \backslash\{w\}$ (figura 4.14(c)). Isto é feito pela função find_node_with_label_b_greater_than_dfs_w (figura 4.17) cuja descrição encontra-se mais adiante. Se existir tal $v$, essa função devolve um apontador para $v$, se $v$ é um P-nó, ou para um filho real de $v$ em $T_{v}^{*}$, caso contrário. ( $\mathrm{O}$ vértice $v$, caso exista, é usado na construção da lista de arcos da obstrução, descrita no capítulo 6.)

Para o caso de dois terminais, digamos $t_{1}$ e $t_{2}$, seja $m$ o ancestral mais baixo de $t_{1}$ e $t_{2}$ em $T$ e sejam $t_{1}^{\prime}$ e $t_{2}^{\prime}$ definidos como a seguir. Para $i=1,2, t_{i}^{\prime}=m$ se $m$ é um P-nó. Caso contrário, $t_{i}^{\prime}$ é o filho real de $m$ que é ancestral de $t_{i}$. (Os cálculos de $t_{i}^{\prime}$ são feitos pela função last_pnode.) 


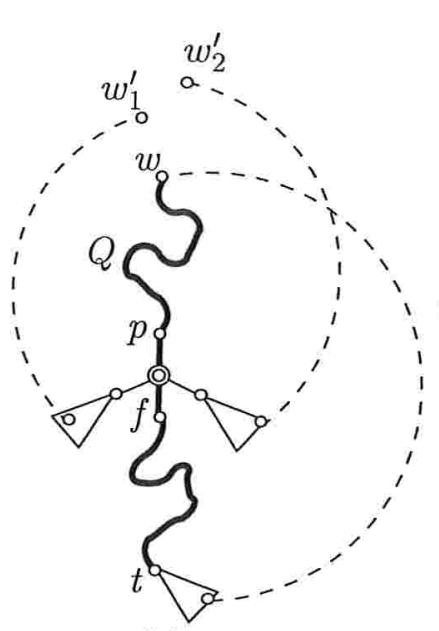

(a)

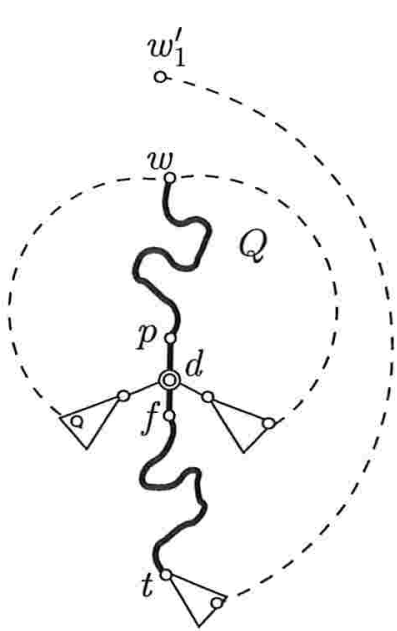

(b)

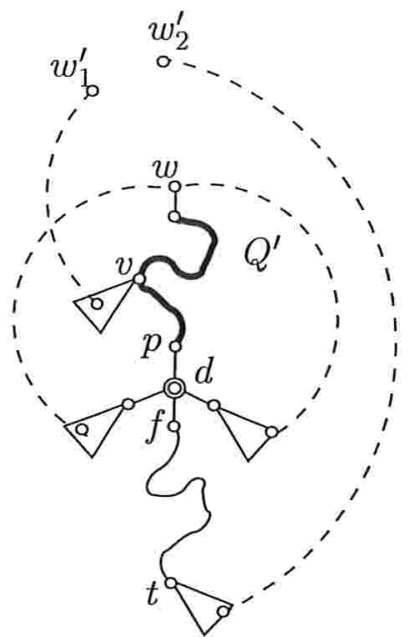

(c)

Figura 4.14: Teste de obstrução para o caso de um terminal $t$, onde $p$ é o pai do C-nó que faz parte de uma obstrução do tipo (o1), $w$ é o vértice correntemente sendo examinado pelo algoritmo de SH e $w_{1}^{\prime}$ e $w_{2}^{\prime}$ são ancestrais de $w$ na PC-árvore. Os caminhos $Q$ e $Q^{\prime}$ são ilustrados por curvas grossas. (a) Uma obstrução do tipo (o1). (b) $d$ é o primeiro C-nó ativo, subindo em $Q$, com dois filhos, em cada um dos lados determinados por $f$ e $p$, com um descendente que é vizinho de $w$. (c) Uma obstrução do tipo (o1).

Seja $Q_{i}$, para $i=1,2$, o caminho de $t_{i}$ a $t_{i}^{\prime}$ em $T$. Analogamente ao caso de um terminal, submete-se $Q_{i}$ à função test_obstruction_from_terminal. Após o teste de cada $Q_{i}$, se a variável global cnode_of_possible_k33_obstruction não é nil, então há uma obstrução do tipo (o1) devida à presença do terminal que não está em $Q_{i}$. Além disso, se $m$ é um C-nó, $m$ é submetido à função test_cnode_counter para verificar se $m$ não faz parte de uma obstrução do tipo (o1).

Finalmente, deve-se testar o caminho $Q^{\prime}$ (figura 4.15), do pai $p$ de $m$ a um filho de $w$, da mesma maneira como descrita para o caso de um terminal.

Para o caso em que há três ou mais terminais num mesmo componente de $T-L$, tem-se uma obstrução do tipo (o3).

\section{Teste de um C-nó}

Testar se um C-nó ativo $d$ faz parte de uma obstrução do tipo (o1) significa, informalmente, determinar se é possível ou não escolher um dos lados do bloco $B_{d}$ na moldura corrente para fazer parte do $X Y$-caminho. Conforme descrito na próxima seção, para cada C-nó ativo $d$, a lista $\mathrm{RBC}(d)$ contém apenas os vértices de $B_{d}$ cujo valor de $b$ é não inferior a $\mathrm{bp}(w)$.

O teste para detectar o caso mais simples de obstrução (figura 4.14(a)) é feito pela função 


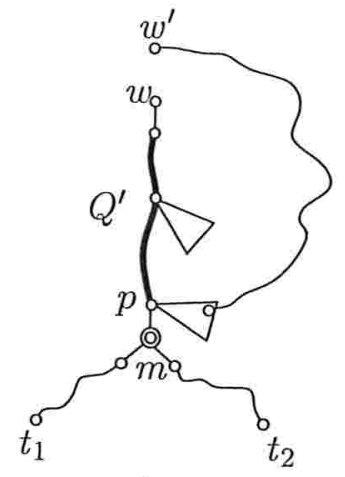

(a)

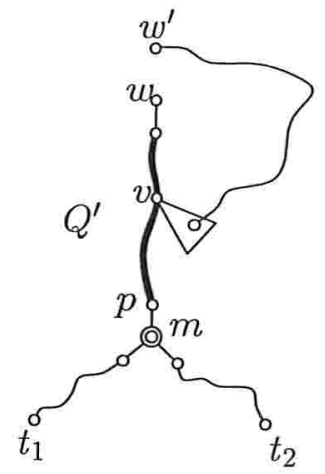

(b)

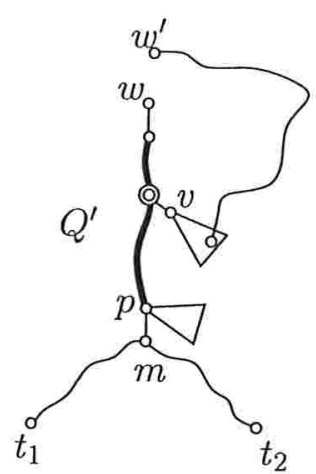

(c)

Figura 4.15: Sejam $t_{1}$ e $t_{2}$ dois terminais, $m$ o ancestral comum de $t_{1}$ e $t_{2}$ em $T$ com menor bp-número, $p$ o pai de $m$ e $w^{\prime}$ um ancestral de $w$. O caminho $Q^{\prime}$ em $T$ é ilustrado por curvas grossas. Em (a) e (b), $m$ é um C-nó que faz parte de uma obstrução do tipo (o2). Em (c) é ilustrada uma situação em que há uma obstrução do tipo (o3).

test_cnode_counter, cujo código se encontra na figura 4.16. Esta função calcula o número de filhos reais de um C-nó ativo $d$, consecutivos e adjacentes ao pai de $d$ no $\operatorname{RBC}(d)$, que possuem um descendente que é um vizinho de $w$. Mais precisamente, dado um c-nó ativo $d$, esta função percorre o $\mathrm{RBC}(d)$, partindo do pai de $d$ (primeiro elemento dessa lista), para os dois lados, até parar num vértice de cada lado, cujo valor de $b$ é superior ao $\mathrm{bp}(w)$. Sejam $l$ e $r$ tais vértices, respectivamente. Durante este processo, calcula-se o número de vértices cujo valor de $b$ é igual ao $\operatorname{bp}(w)$. Se $l$ e $r$ são ancestrais de terminais, então eles também são contabilizados na soma. Ao final da função, a soma obtida é comparada com o valor de counter[d]. Caso a soma seja diferente de counter[d], uma obstrução do tipo (o1) foi detectada.

Somente com a função test_cnode_counter, o algoritmo não consegue detectar o caso ilustrado na figura 4.14(c). Para lidar com este caso, faz-se a variável global cnode_of_possible_k33_obstruction apontar para o primeiro C-nó em $Q$, subindo em $T$, onde ocorre a situação ilustrada na figura 4.14(b), que é detectada pela função test_cnode_counter quando os dois lados do RBC são percorridos durante a contagem dos filhos. Neste caso, é preciso fazer uma verificação em $Q^{\prime}$, conforme é descrito a seguir.

\section{Teste do caminho $Q^{\prime}$}

O teste do caminho $Q^{\prime}$ para o caso de um ou dois terminais (figuras 4.14(b) e 4.15, respectivamente) é feito pela função find_node_with_label_b_greater_than_dfs_w, cujo código se encontra na figura 4.17.

Para cada nó $u$ em $Q^{\prime}$, seja $u^{\prime}$ o filho de $u$ no caminho $Q$. Esta função faz com que $u^{\prime}$ deixe 
〈teste de um c-nó 68〉三

bool sh_planar_impl::test_cnode_counter(graph\& $G$, node cnode, node $w$, node $w 1$, node $w 2)$

$\{$ if $(R B C[$ cnode].size ()$\leq 2)$ return false;

bmlist_item $i t 1=R B C\left[\right.$ cnode].first_item ()$, i t 1 l=R B C[$ cnode $] . c y c l i c \_p r e d(i t 1$, nil $)$, $i t 1 r=R B C[$ cnode $]$. cyclic_succ $(i t 1$, nil $) ;$

node $l=R B C[$ cnode].contents(it1l), $r=R B C[$ cnode $] . \operatorname{contents}(i t 1 r), u l=$ nil, $u r=$ nil;

int count $=1 ; \quad / /$ starts counting parent[cnode];

bmlist_item $a, s=i t 1$;

while (label_b $\left.[l] \equiv d f s p o s \_n u m[w]\right) / /$ goes to the left; (2)

$\{u l=l$; count $+; a=i t 1 l$;

it $1 l=R B C[$ cnode $] . c y c l i c \_p r e d(i t 1 l, s) ; s=a$;

$l=R B C[$ cnode].contents $($ it $1 l)$;

\}

bmlist_item $p=i t 1$;

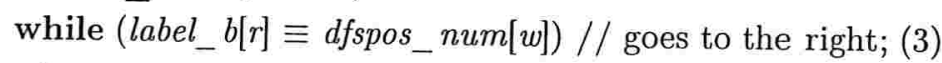

$\{u r=r$ count $+; a=i t 1 r$;

$i t 1 r=R B C[$ cnode $] . c y c l i c \_s u c c(i t 1 r, p) ; p=a$;

$r=R B C[$ cnode $]$.contents $(i t 1 r)$;

\}

if $(l \equiv w 1 \vee l \equiv w 2)$ count + ;

if $(r \neq l \wedge(r \equiv w 1 \vee r \equiv w 2))$ count + ;

if (count < counter [cnode]) return true;

node $f=$ nil;

if $(u l \neq \operatorname{nil} \wedge u r \neq$ nil $)$

$f=l ; / /$ or $\mathrm{f}=\mathrm{r}$;

else if $(u l \neq \operatorname{nil} \wedge w 1 \neq \operatorname{nil} \wedge w 1 \neq l)$

$\{f=l ; u r=w 1 ;\}$

else if $(u r \neq \operatorname{nil} \wedge w 1 \neq \operatorname{nil} \wedge w 1 \neq r)$

$\{f=r ; u l=w 1 ;\}$

if (cnode_of_possible_k33_obstruction $\equiv$ nil $\wedge f \neq$ nil)

cnode_of_possible_k33_obstruction $=$ cnode ;

return false;

\}

Figura 4.16: Código da função test_cnode_counter. 


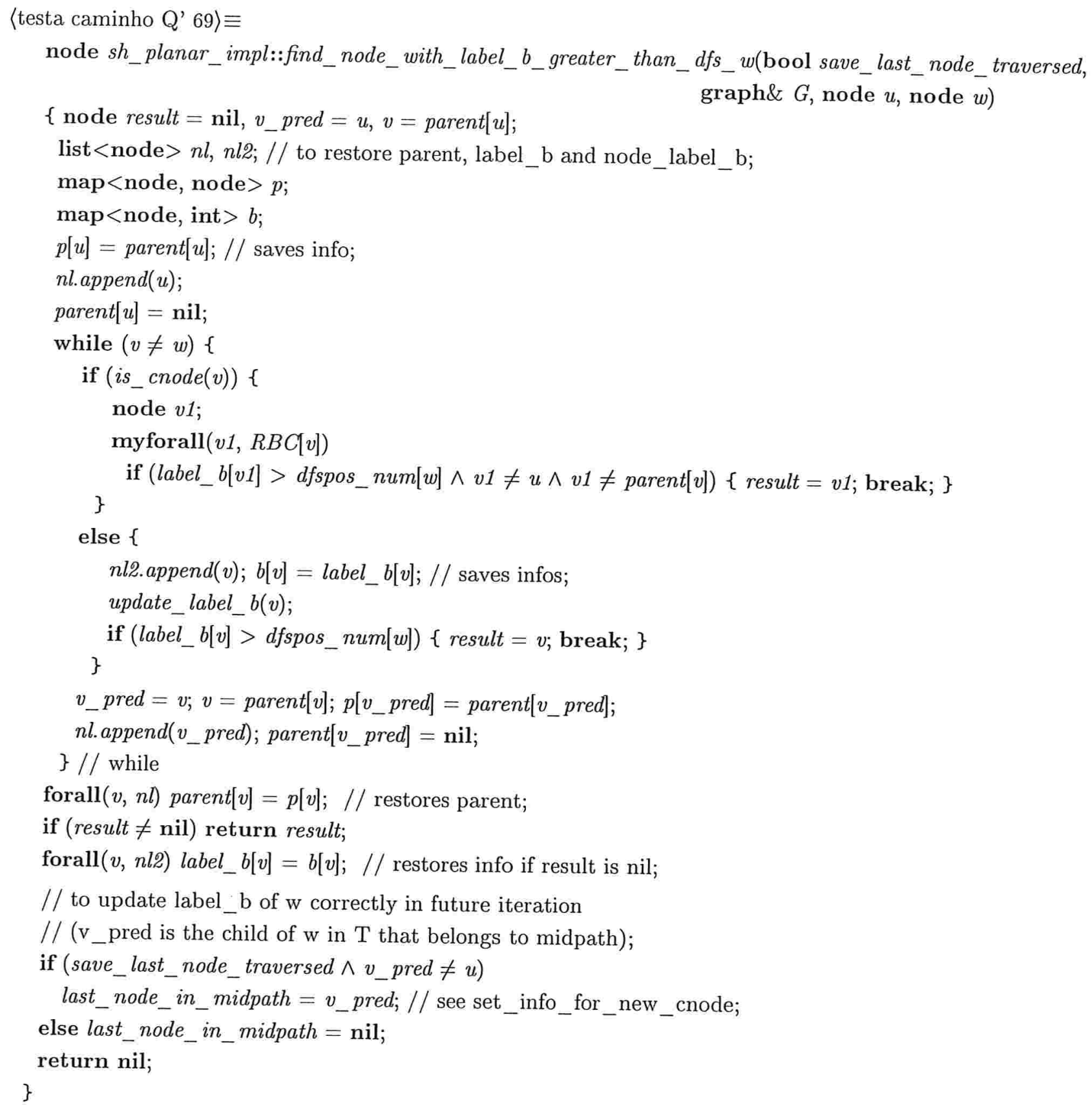

Figura 4.17: Código da função find_node_with_label_b_greater_than_dfs_w. 
de ser, temporariamente, um filho de $u$. Se $u$ é um P-nó, então o valor de $b(u)$ é recalculado. Se o novo valor $b(u)$ é superior ao $\mathrm{bp}(w)$, então a função devolve este P-nó. Se $u$ é um C-nó, então a função devolve, caso exista, um P-nó $v$ que é filho de $u$ tal que $b(v)>\operatorname{bp}(w)$ e $v$ não está em $Q$. Ao final da função, os pais e valores de $b$ são restaurados.

A variável global last_node_in_midpath guarda o último nó de $Q^{\prime}$ quando save_last_node_traversed é verdadeiro. Isto é importante para que o recálculo dos valores de $b$, feitos pela rotina update_label_b, cujo código é ilustrado na figura 4.18, funcione corretamente. A variável last_node_in_midpath é usada durante o cálculo do RBC para um novo C-nó, explicado na próxima seção.

\section{Cálculo dos valores de $b$}

À medida em que a PC-árvore é atualizada durante a execução do algoritmo de SH, é necessário atualizar-se os valores de $b$ para os vértices que farão parte do $\mathrm{RBC}$ do novo C-nó. Isto é feito pela rotina update_label_b (figura 4.18).

$\langle$ atualiza rótulos b 70$\rangle \equiv$

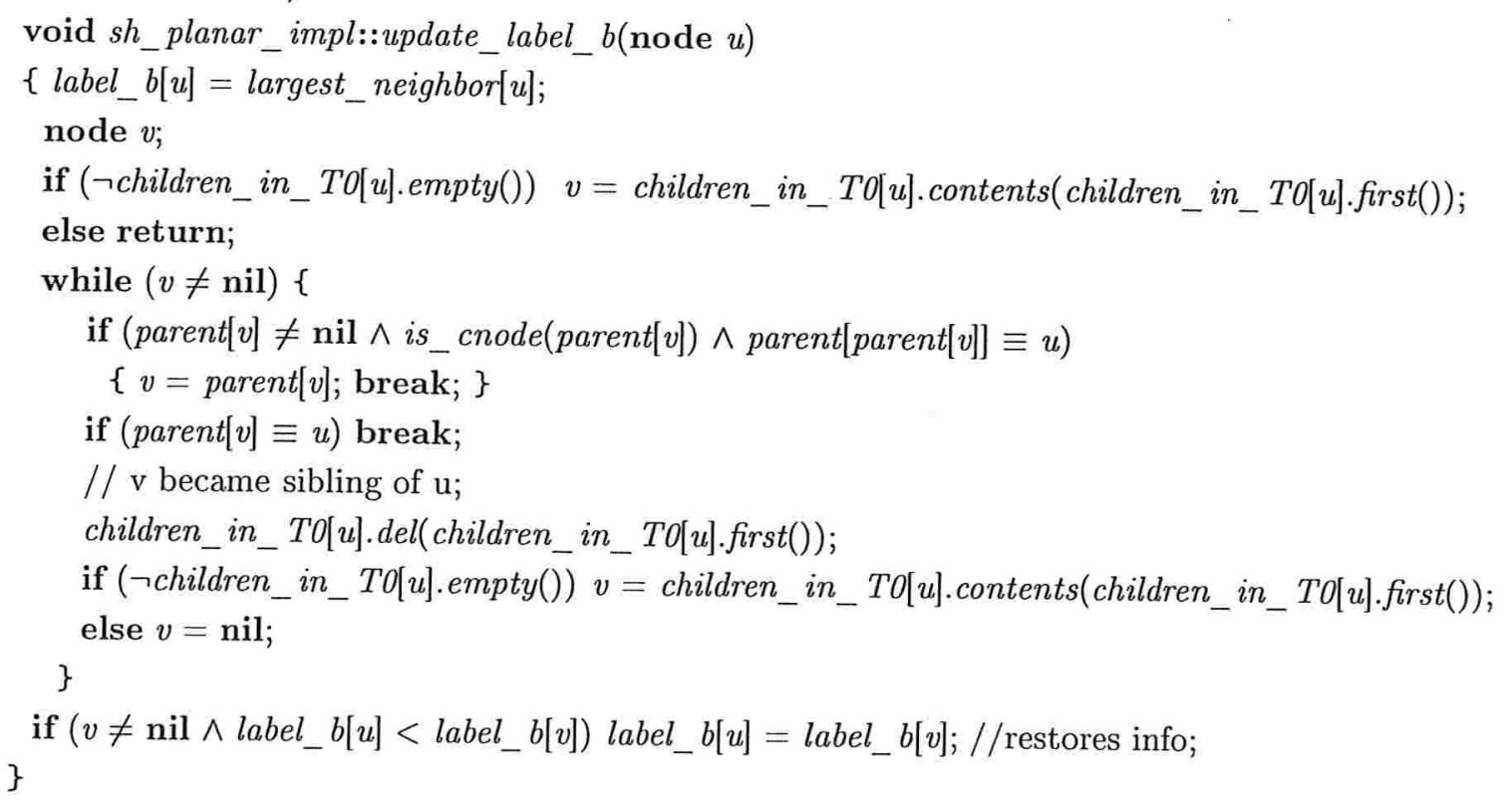

Figura 4.18: Código da função update_label_b.

Para que esse processo seja eficiente, uma lista dos filhos de cada vértice na árvore $T_{0}$, em ordem não-decrescente do valor de $b$, é construída durante o pré-processamento. Esta lista é dada pela variável global children_in_TO[ ]. A idéia é que, para cada vértice $u$, os vértices de children_in_TO[u] que se tornaram irmãos de $u$ na PC-árvore corrente $T$ são removidos de 
children_in_To[u]. Seja $v$ o primeiro elemento dessa lista após a remoção dos vértices que se tornaram irmãos de $u$, caso exista. Na atualização, se $b(v)>h(u)$, então $b(u)$ passa a ser $b(v)$. Se a lista children_in_TO[u] está vazia ou $b(v) \leq h(u)$, então $b(u)$ passa a ser $h(u)$.

\subsection{Construção de um novo C-nó}

Neste ponto do algoritmo, um componente $K$ de $T-L$ com um ou dois terminais está em foco e sabe-se que existe um $X Y$-caminho $P$ em $K$. Essa fase consiste na criação de um C-nó $c$ para representar o bloco $B_{c}$ de $H+w$ que contém vértices de $K$. Ela consiste em duas tarefas: a atualização da árvore $T$ e do vetor $b$ e a construção do $\operatorname{RBC}(c)$. Tanto a atualização de $T$ e de $b$ quanto a construção de $\mathrm{RBC}(c)$ são feitas pela rotina set_info_for_new_cnode, cujo código é ilustrado na figura 4.19 .

void sh_planar_impl::set_info_for_new_cnode(graph\& $G$, node $w$, node new_cnode,

$\left\{\right.$ label_b $[$ new_cnode $]=d f s p o s \_n u m[w] ;$

list $<$ node $>$ \& terminal_nodes $)$

state $[$ new_cnode $]=N O T_{-}$VISITED;

counter [new_cnode $]=0$;

parent $[$ new_cnode $]=w$;

calculate_new_RBC( $G$, new_cnode, $w$, terminal_nodes $)$;

if (last_node_in_midpath $\neq$ nil) \{

parent[last_node_in_midpath $]=$ new_cnode;

last_node_in_midpath $=\mathbf{n i l} ; / /$ resets global variable; \}

RBC[new_cnode].push $(w)$;

bmlist_item first $=R B C\left[n e w_{-}\right.$cnode $] . f i r s t_{-}$item (), last $=R B C\left[n e w_{-}\right.$cnode $] . l a s$ latem $\left._{(}\right)$;

activecnode $[$ first $]=$ new_cnode;

activecnode $[$ last $]=$ new_cnode;

\}

Figura 4.19: Código da função set_info_for_new_cnode.

Essa função inicializa os valores de b, statel ] e counter [ / do novo C-nó e faz $w$ ser o pai desse C-nó. Em seguida, calcula o RBC para o novo C-nó através da função calculate_new_ $R B C$ (figura 4.20), descrita mais adiante. As atualizações de $T$ e de $b$ são feitas durante o cálculo do novo RBC. Feito isso, se o componente $K$ tem exatamente dois terminais, então a variável last_node_in_midpath aponta para o último vértice de $Q^{\prime}$, caso exista. Se existir tal vértice, seu pai passa a apontar para o novo C-nó. Isto é necessário para que a rotina update_label_b funcione corretamente. 
Finalmente, $w$ é inserido no início do novo RBC e, para cada uma das pontas dessa lista, a variável global activecnodel / é atualizada ou inicializada, passando a apontar para o novo C-nó.

\section{Cálculo do novo RBC e atualização de $T$ e de $b$}

O cálculo do novo RBC e a atualização de $T$ e de $b$ são feitos pela função calculate_new_ $R B C$ (figura 4.20). Na implementação aqui apresentada, tem-se o seguinte invariante: para cada C-nó ativo $c$, a lista $\operatorname{RBC}(c)$ contém apenas os vértices de $B_{c}$ cujo valor de $b$ é maior que $\mathrm{bp}(w)$.

Para o caso de um terminal $t$ em $K$, seja $Q$ o caminho de $t$ a $w$ em $T$. O cálculo do novo $\mathrm{RBC}$ é feito através da função calc_new_RBC_from_terminal_node (figura 4.21). Para cada nó $v$ em $Q$, subindo em $T$, se $v$ é um P-nó, então seu pai passa a apontar para o novo C-nó e $b(v)$ é recalculado pela rotina update_label_b. Se o novo valor de $b(v)$ é maior do que $\mathrm{bp}(w)$, então $v$ é inserido no fim do novo RBC e sua posição nessa lista é guardada em ptritem[v].

Se $v$ é um C-nó, então a rotina $a d d_{-}$old_cnode_ $R B C_{-} t_{-} o_{-} n e w_{-} R B C$ (figuras 4.22 e 4.23 ) é acionada. Ela "compacta" o $\mathrm{RBC}(v)$ e depois verifica se é preciso inverter essa lista antes de concatená-la ao final do novo $\mathrm{RBC}$. Observe que, para cada vértice $u$ do $\mathrm{RBC}(v)$ compactado, não é preciso recalcular ptritem [u].

Para a compactação do $\mathrm{RBC}(v)$, um processo similar à função test_cnode_counter é realizado: o $\mathrm{RBC}(v)$ é percorrido, partindo-se do pai de $v$ (primeiro elemento dessa lista) para os dois lados, até encontrar um vértice de cada lado cujo $b$ é maior que $\mathrm{bp}(w)$. (Se $u$ é um ancestral de um terminal em $\operatorname{RBC}(v)$, então seu $b$ já está atualizado para o novo C-nó, por isso foi necessário incluir as condições (1) e (2) nas figuras 4.22 e 4.23.) O pai de $v$ e os vértices percorridos nesse processo cujo $b \leq \mathrm{bp}(w)$ são removidos do $\operatorname{RBC}(v)$. Se $u$ é um ancestral de um terminal em $\operatorname{RBC}(v)$, então $u$ também é removido do $\operatorname{RBC}(v)$, pois ele foi inserido no $\mathrm{RBC}$ do novo C-nó se o novo valor de $b(u)$ é maior que $\mathrm{bp}(w)$.

Para verificar se é preciso inverter o $\operatorname{RBC}(v)$ antes da concatenação, se $v$ é um terminal, então não é preciso fazer nada. (Na verdade, na implementação aqui apresentada, se $v$ é um terminal e algum vértice à "esquerda" do pai de $v$ (figura 4.24(a)) foi removido de $\mathrm{RBC}(v)$, então essa lista é invertida para manter a consistência com o que é feito no capítulo 5 para a construção da descrição combinatória plana.) Caso contrário, seja $f$ o vértice em $\operatorname{RBC}(v)$ que está em $Q$. Se $f$ é o último elemento de $\operatorname{RBC}(v)$ então essa lista é invertida (figura 4.24(c)). No final da função, o $\mathrm{RBC}(v)$ é concatenado no fim do novo RBC.

Para o caso de dois terminais (usando a mesma notação usada anteriormente), de forma análoga ao caso de um terminal, calcula-se os dois "pedaços" do novo RBC (atualizando $T$ e $b$ ), devidos aos caminhos $Q_{1}$ e $Q_{2}$, através da função calc_new_RBC_from_terminal_node. Em seguida, $m$ é adicionado ao novo RBC de maneira apropriada e o pai de $m$ passa a apontar para 
$\langle$ constrói RBC do novo c-nó 73$\rangle \equiv$

void $s h \_p l a n a r+i m p l:$ :calculate_new_RBC(graph\& $G$, node $n e w \_c n o d e$, node $w$,

$\{$ switch(terminal_nodes.size ()$)\{$

list $<$ node $>$ \& terminal_nodes $)$

case 1:

$\{$ node $t 1=$ terminal_nodes.pop ()$;$ \} $c a l c_{-} n e w_{-} R B C_{-} f r o m_{-} t e r m i n a l \_n o d e\left(n e w_{-} c n o d e, w, t 1, w, R B C\left[n e w_{-}\right.\right.$cnode $\left.]\right) ;$ break;

case 2:

$\{$ node $m=$ nil, $t 1=$ terminal_nodes.pop ()$, t 2=$ terminal_nodes.pop () , aux $1=t 1$, aux $2=t 2$

// makes t1 with lower dfspos_num than $\mathrm{t} 2$;

if $($ is_cnode $(\operatorname{aux1}))$ aux1 = parent $[$ aux1 $]$;

if (is_cnode(aux2)) aux2 = parent [aux2];

if (dfspos_num[aux1] >dfspos_num[aux2]) leda_swap(t1, t2);

$m=l c a \_b e t w e e n \_t e r m \_n o d e s(t 1, t 2)$;

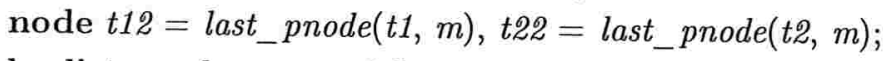

bmlist $<$ node $>$ auxnodelist;

calc_new_RBC_from_terminal_node(new_cnode, $w, t 1$, t12, auxnodelist);

calc_new_RBC_from_terminal_node(new_cnode, $\left.w, t 2, t 22, R B C\left[n e w \_c n o d e\right]\right)$;

// adds $\mathrm{m}$ to $\mathrm{RBC}[$ new_cnode];

if $($ is_cnode $(m))$ \{

$m=$ active_cnode_of(false, $m)$;

parent $[$ t12 $]=$ parent $[$ t22 $]=$ new_cnode;

update_label_b(t12); update_label_b(t22);

if (label_b[t12] $>$ dfspos_num[w]) ptritem[t12] = auxnodelist.append(t12);

if (label_b[t22] $\left.>d f s p o s \_n u m[w]\right)$ ptritem[t22] $=R B C\left[n e w \_c n o d e\right]$. append(t22);

add_old_cnode_RBC_to_new_RBC(m,new_cnode, $\left.w, t 22, t 12, R B C\left[n e w_{-} c n o d e\right]\right)$; parent $[m]=$ new cnode;

\}

else \{

parent $[m]=n e w \_c n o d e ;$ update_label_b $(m)$;

if $\left(\right.$ label_b $\left.[m]>d f s p o s_{-} n u m[w]\right)$ ptritem $[m]=R B C\left[n e w \_\right.$cnode $]$. append $(m)$; \}

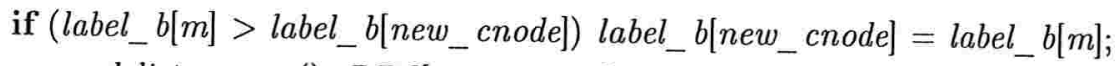
auxnodelist.reverse (); RBC[new_cnode].conc(auxnodelist);

\}

break;

\}

\}

Figura 4.20: Código da função calculate_new_ $R B C$. 


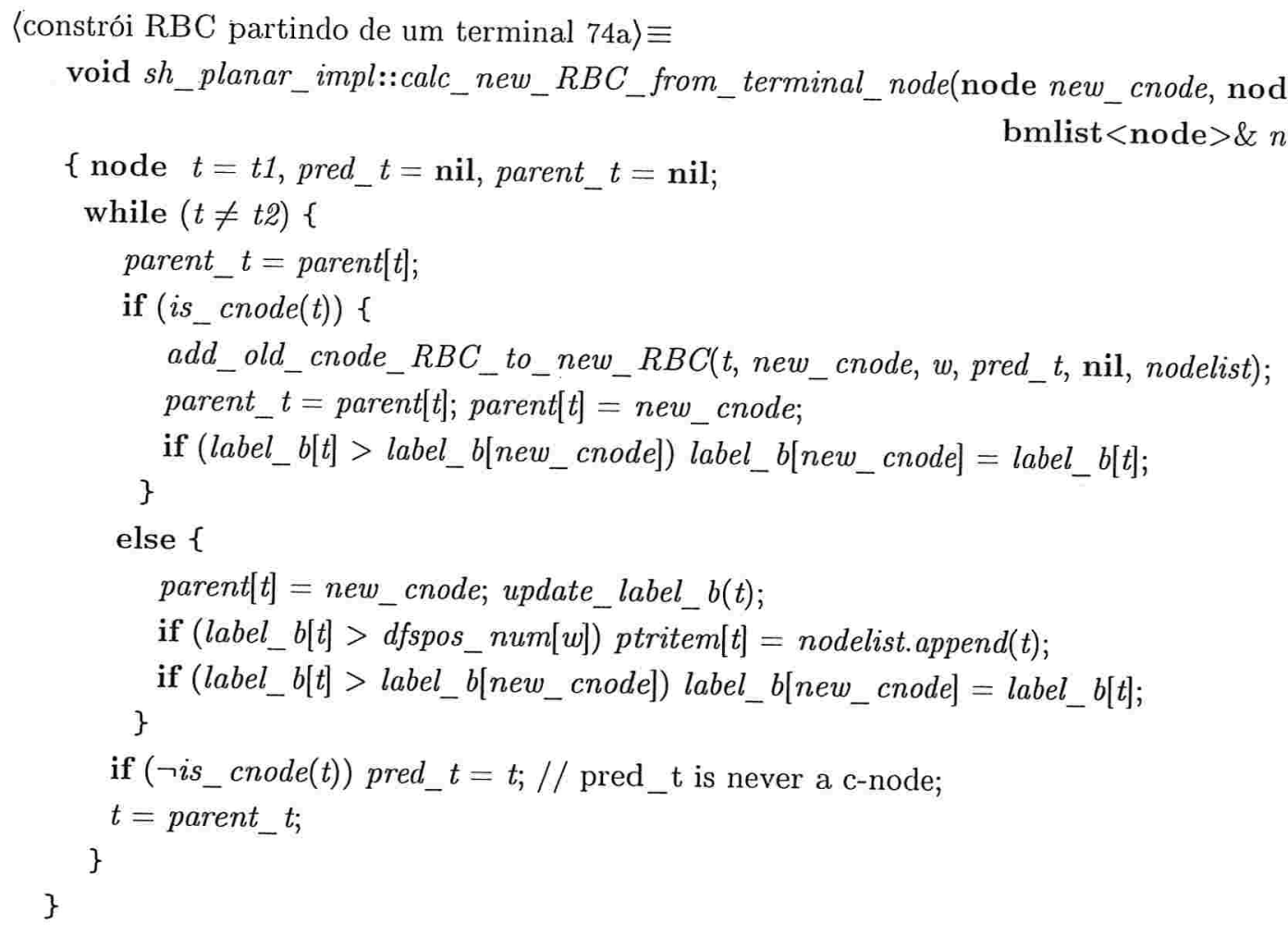

Figura 4.21: Código da função calc_new_RBC_from_terminal_node.

void $s h \_p l a n a r_{-} i m p l:: a d d_{-} o l d \_c n o d e_{-} R B C_{-} t o_{-} n e w_{-} R B C($ node oldcnode, node newcnode, node $w$,

\{ // compress RBC[oldcnode]; node $w 1$, node $w 2$, bmlist $<$ node $>$ \& nodelist)

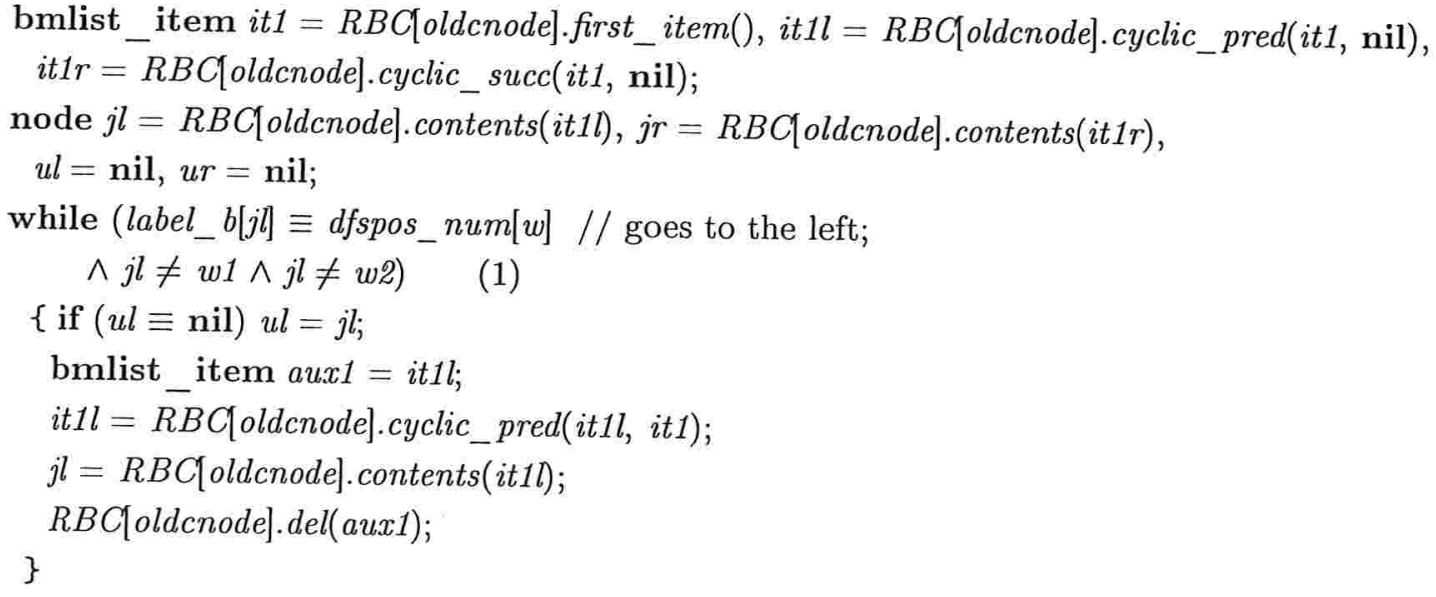

Figura 4.22: Código da função add_old_cnode_ $R B C_{-}{ } o_{-} n e w_{-} R B C$. 
while (label_b $b r] \equiv d f s p o s \_n u m[w] / /$ goes to the right;

$\wedge j r \neq w 1 \wedge j r \neq w 2)$

$\{$ if $(u r \equiv$ nil) $u r=j r$;

bmlist_item aux1 $=i t 1 r$;

$i t 1 r=\bar{R} B C[$ oldcnode $]$. cyclic_succ $(i t 1 r, i t 1)$;

$j r=R B C[$ oldcnode].contents $(i t 1 r)$;

$R B C[o l d c n o d e] . \operatorname{del}($ aux 1$)$;

\}

RBC[oldcnode].del(RBC[oldcnode].first_item ()$)$;

node first $=w 1$;

if $(w 1 \equiv$ nil $)$

if $(u l \neq$ nil $)$ first $=j l$;

else first $=j r$;

// checks if RBC[oldcnode] flipped;

if $(R B C[$ oldcnode].contents $(R B C[$ oldcnode].last_item ()$) \equiv$ first $)$ RBC[oldcnode].reverse () ;

// removes w1 and w2 from $\mathrm{RBC}[$ oldcnode];

if ( $w 1 \neq$ nil) RBC[oldcnode].del(RBC[oldcnode].first_item());

if (w2 $\neq$ nil) RBC[oldcnode].del(RBC[oldcnode].last_item());

nodelist.conc(RBC[oldcnode]);

\}

Figura 4.23: (Continuação) Código da função add_old_cnode_ $R B C_{-}$to_new_ $R B C$.

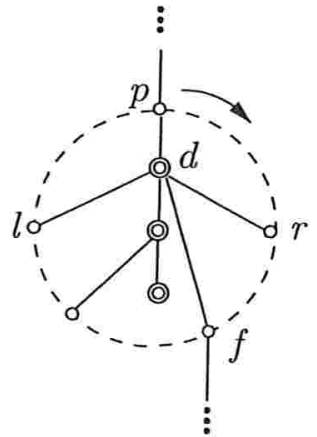

(a)

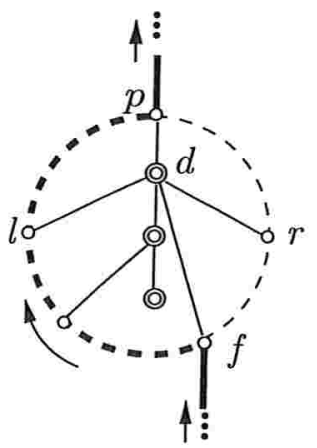

(b)

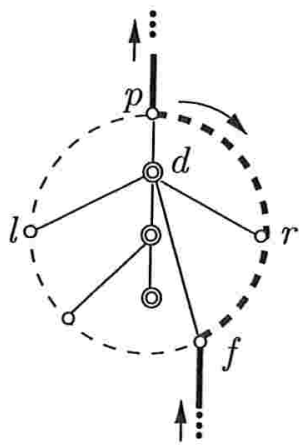

(c)

Figura 4.24: Os vértices $l$ e $r$ em $\operatorname{RBC}(d)$ estão a esquerda e a direita de $p$, respectivamente, onde $p$ é o pai de $d$ em $T$. (a) Os elementos de $\mathrm{RBC}(d)$ estão no sentido horário. (b) O lado direito de $\operatorname{RBC}(d)$ é removido durante a compactação de $\operatorname{RBC}(d)$. (c) $\mathrm{O}$ lado esquerdo de $\mathrm{RBC}(d)$ é removido na compactação e é necessário inverter $\operatorname{RBC}(d)$. 
o novo C-nó. Se $m$ é um C-nó, então os valores de $b\left(t_{1}^{\prime}\right)$ e $b\left(t_{2}^{\prime}\right)$ são recalculados. Se $m$ é um P-nó, então $b(m)$ é recalculado e ptritem $/ m]$ guarda a posição de $m$ no novo RBC. Observe que um dos pedaços, devido a $Q_{1}$ ou a $Q_{2}$, do novo $\mathrm{RBC}$ deve ser invertido. (Na implementação aqui apresentada, $t_{1}$ é o terminal de menor bp-número e o pedaço do novo $\mathrm{RBC}$ devido a $Q_{1}$ foi invertido. Tudo isso foi necessário para manter a consistência com o que é feito no capítulo 5 , para a construção da descrição combinatória plana.)

Uma simulação da PC-árvore alterada para o grafo da figura 4.1(a) é ilustrada nas figuras 4.25 e 4.26, onde, para cada C-nó ativo $c$, somente os filhos reais de $c$ que estão no $\mathrm{RBC}(c)$ estão ilustrados. Os filhos reais de $c$ que foram omitidos do $\mathrm{RBC}(c)$ também são omitidos nas figuras.

\subsection{Correção}

A correção baseia-se na validade dos seguintes invariantes envolvendo o grafo $G$, o conjunto $L$ e a árvore $T$ mantida pelo algoritmo:

(ish1) o subgrafo $G[L]$ é conexo;

(ish2) $T$ é uma PC-árvore alterada associada a uma moldura de $G\left[V_{G} \backslash L\right]$ em $G$.

O primeiro invariante decorre facilmente do fato de que os vértices são processados de acordo com uma bp-ordenação.

Para mostrar a validade do segundo invariante, deve-se verificar que, ao final de uma iteração do algoritmo de SH, ou uma $X Y$-obstrução é detectada ou $T$ é uma PC-árvore alterada associada a uma moldura de $G\left[V_{G} \backslash L^{\prime}\right]$ em $G$.

O algoritmo modifica a árvore $T$, por meio da função find_active_cnode, enquanto busca um $X Y$-caminho ou uma $X Y$-obstrução. Nessa função, são feitas apenas modificações no valor de parent [u], que passa a apontar para o pai real de um P-nó u. Claramente tais modificações não afetam a validade do invariante (ish2).

A função que verifica a existência de uma $X Y$-obstrução é a função find_obstruction. Os lemas a seguir mostram que essa função devolve true se e somente se existe uma $X Y$-obstrução no componente em questão da moldura $M$.

Lema 4.2: Se o algoritmo atinge um dos pontos marcados com (1) nas funções find_obstruction e test_obstruction_from_terminal (figuras 4.11 e 4.13, nas páginas 63 e 65, respectivamente), há uma obstrução do tipo (o1) em $M$.

Demonstração: Considere o caso da função test_obstruction_from_terminal, onde uma obs- 


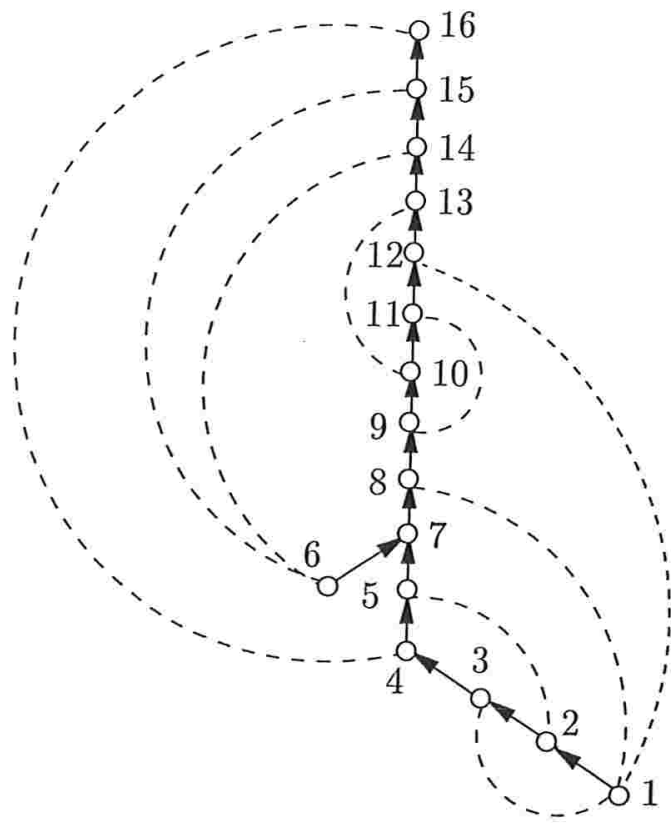

(a)

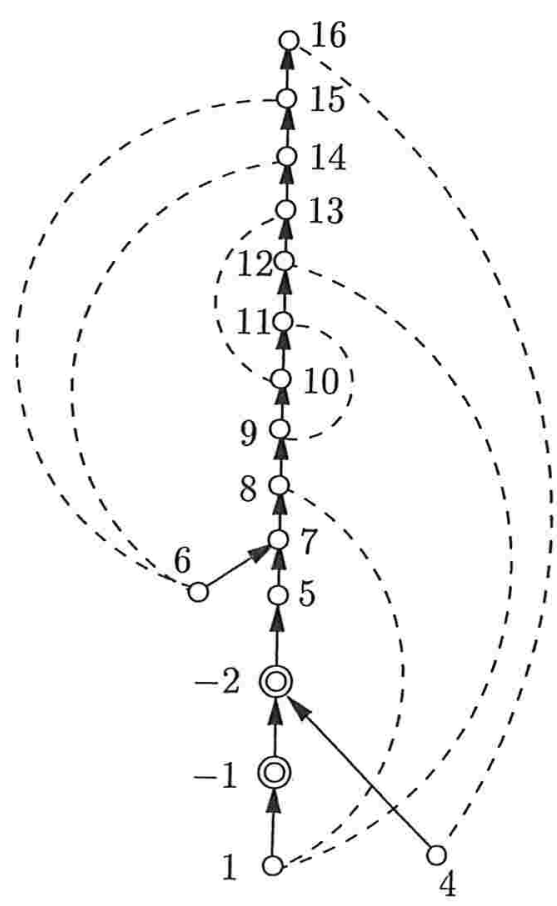

(c)

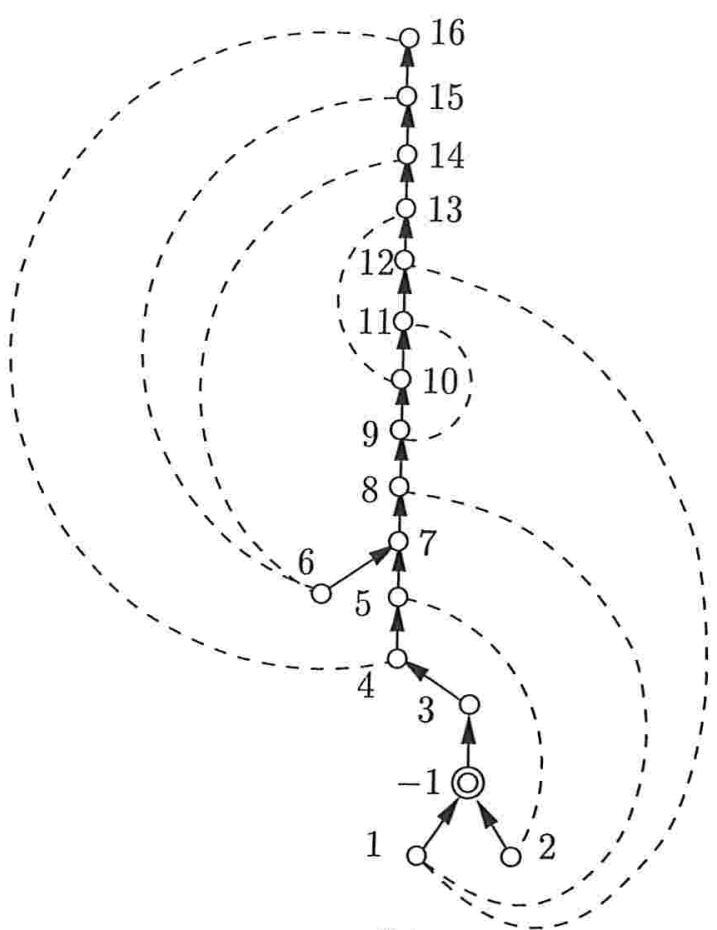

(b)

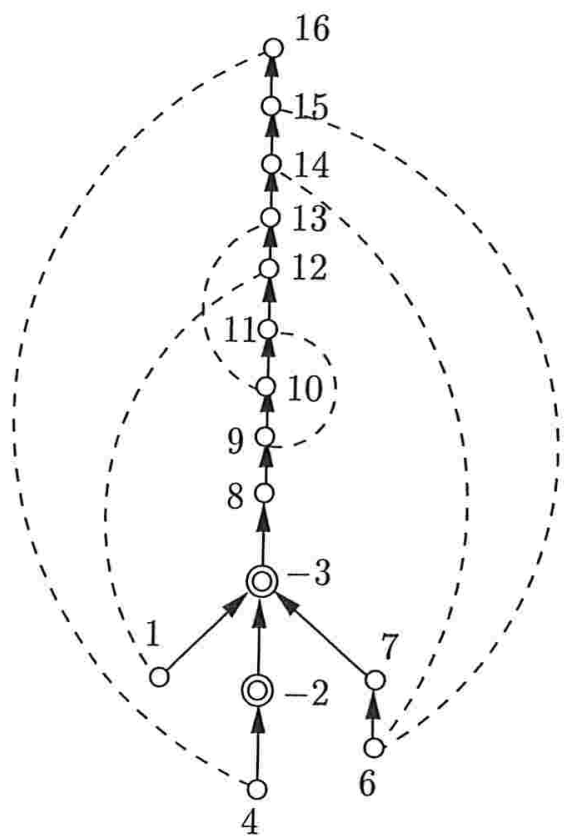

(d)

Figura 4.25: Execução do algoritmo sobre o grafo da figura 4.1(a). (a) Configuração inicial da PC-árvore alterada $T$. (b) $T$ após o algoritmo examinar o vértice 3. (c) $T$ após examinar 5 . (d) $T$ após examinar 8. 


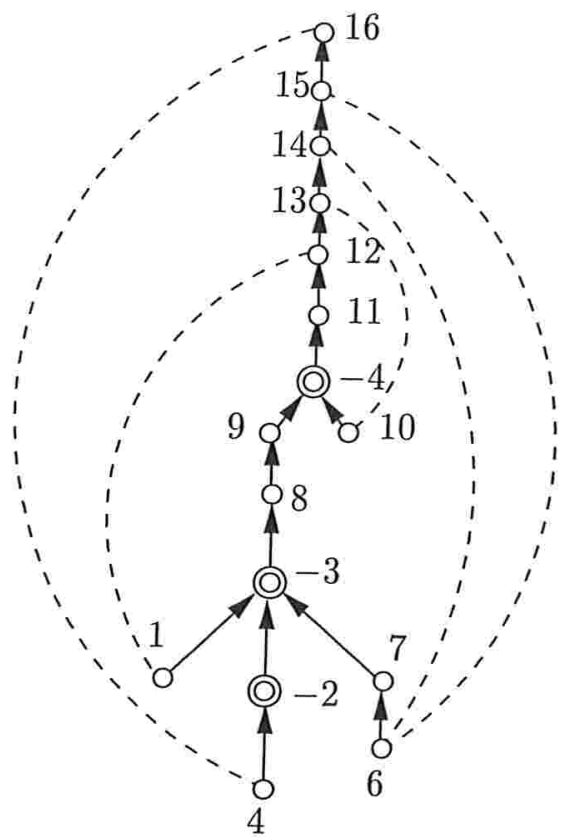

(e)

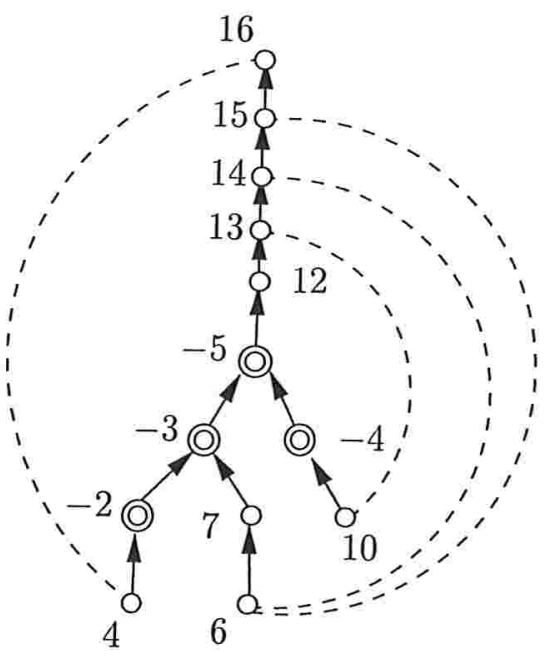

(f)

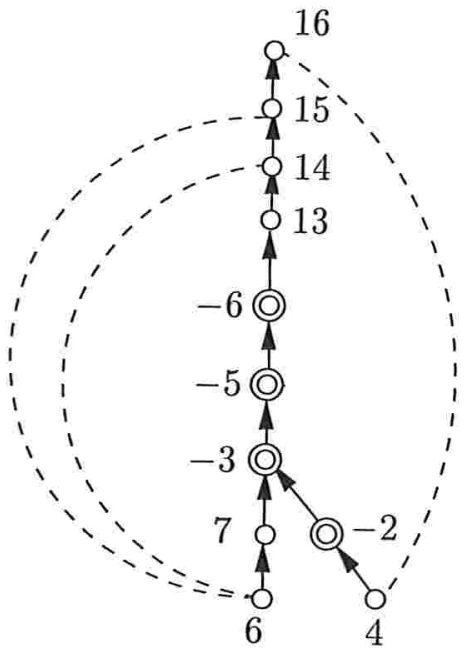

(g)

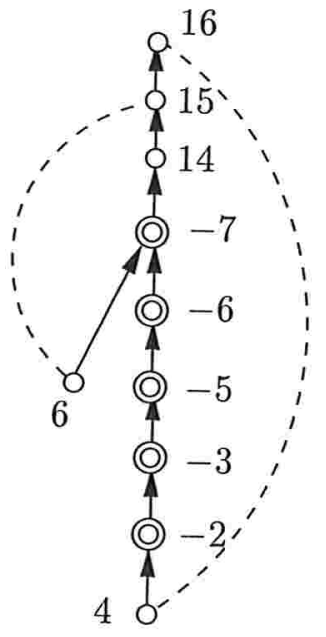

(h)

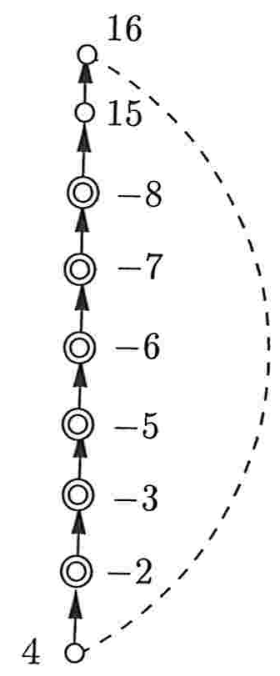

(i)

Figura 4.26: (Continuação) Execução do algoritmo sobre o grafo da figura 4.1(a). (e) $T$ após examinar 11. (f) $T$ após examinar 12. (g) $T$ após examinar 13. (h) $T$ após examinar 14. (i) $T$ após examinar 15. 
trução é detectada quando, na função test_cnode_counter (figura 4.16), a condição (1) é verdadeira. Seja $c$ um C-nó ativo no caminho $Q$. Lembre-se que a variável counter [c] contém o número de filhos reais de $c$ que enxergam $X$ através de $\left(V_{G} \backslash L\right) \backslash V_{B_{c}}$ e note que, claramente, count $\leq$ counter $[c]$, onde count é o contador da função test_cnode_counter. Caso estes dois contadores não coincidam, há uma obstrução do tipo (o1), conforme mostrado a seguir. Seja $p$ o pai de $c$ em $T$. Neste caso, existe um filho real $f$ de $c$ que não foi contado em count e $f$ está entre dois vértices no $R B C(c)$, digamos $l$ e $r$, que são distintos entre si e distintos de $p$, e que enxergam $Y$ através de $\left(V_{G} \backslash L\right) \backslash V_{B_{c}}$. Além disso, $f$ está no lado oposto a $p$ no $\operatorname{RBC}(c)$ e a $X Y$-obstrução encontrada é $(C, p, l, f, r)$, onde $C$ é o bloco $B_{c}$ ( $B_{c}$ é o bloco de $M$ correspondente a $c$, que é o ciclo facial externo de $c$, definido no próximo capítulo) (figura 4.14, página 66).

O próximo lema diz respeito aos casos marcados com (2) na função find_obstruction, que é parcialmente detectado pela função test_cnode_counter, que modifica o conteúdo da variável global cnode_of_possible_k33_obstruction.

Lema 4.3: Se o algoritmo atinge um dos pontos marcados com (2) na função find_obstruction (figuras 4.11 e 4.12, páginas 63 e 64), então há uma obstrução do tipo (o1) em $M$.

Demonstração: Para ocorrer este caso, a variável cnode_of_possible_k33_obstruction foi modificada pela função test_cnode_counter e aponta para um C-nó ativo $\bar{d}$ como ilustrado na figura 4.14(b). Seja $p$ o pai de $d$ em $T$. Neste caso, $d$ tem três filhos reais $l, r$ e $f$, distintos entre si, tais que $f$ enxerga $Y$ através de $\left(V_{G} \backslash L\right) \backslash V_{B_{d}}$ e $l$ e $r$ enxergam $X$ através de $\left(V_{G} \backslash L\right) \backslash V_{B_{d}}$.

Claramente, tanto para o caso de um terminal como para o caso de dois terminais, as marcas (2) são atingidas somente se $p$ enxerga $Y$ através de $\left(V_{G} \backslash L\right) \backslash V_{B_{d}}$. Logo, a $X Y$-obstrução encontrada neste caso é $(C, p, l, f, r)$, onde $C$ é o bloco $B_{d}$.

A situação em que uma obstrução é detectada no ponto marcado com (3) na função find_obstruction é analisada no lema abaixo.

Lema 4.4: Se o algoritmo atinge o ponto marcado com (3) na função find_obstruction

(figura 4.12, página 64), então há uma obstrução do tipo (o2) ou do tipo (o3) em $M$.

Demonstração: Este caso se manifesta num componente de $T-L$, correspondente a um componente $K$ em $M$, com dois terminais, digamos $t_{1}$ e $t_{2}$. Seja $m$ o ancestral comum entre $t_{1}$ e $t_{2}$ em $T$ com o menor bp-número, seja $Q$ o caminho em $T$ de um dos terminais a $w$ e seja $Q^{\prime}$ o subcaminho de $Q$, do pai de $m$ ao filho de $w$.

Para um nó $v$ em $Q^{\prime}$, seja $T_{v}^{*}:=T_{v}-T_{v^{\prime}}$, onde $v^{\prime}$ é o filho de $v$ em $Q$ e seja $V^{*}$ o conjunto 
dos P-nós em $T_{v}^{*}$ a menos de $v$. Se o algoritmo atinge o ponto marcado com (3), então existe $v$ tal que $v$ é ou um P-nó em $Q^{\prime}$ ou um filho real de um C-nó em $Q^{\prime}$ tal que $v$ enxerga $L$ através de $V^{*}$.

Se $m$ é um C-nó (ativo) então, para $i=1,2$, seja $t_{i}^{\prime}$ o filho real de $m$ que é ancestral de $t_{i}$. Sejam $p$ o pai de $m$ e $C=B_{m}$. A obstrução $\left(C, t_{1}^{\prime}, t_{2}^{\prime}, p\right)$ é do tipo (o2).

Se $m$ é um P-nó então sejam $K_{1}, K_{2}$ e $K_{3}$ os componentes resultantes de $K-m$ que contém, respectivamente, $t_{1}, t_{2}$ e $v$. Neste caso, $\left(m, K_{1}, K_{2}, K_{3}\right)$ é uma obstrução do tipo (o3).

Finalmente, a última situação em que uma obstrução é detectada pelo algoritmo é descrita no lema abaixo.

Lema 4.5: Se o algoritmo atinge o ponto marcado com (4) na função find_obstruction

(figura 4.12, página 64), então há uma obstrução do tipo (o2) ou do tipo (o3) em $M$.

Demonstração: Este caso ocorre quando existem três ou mais terminais num componente de $T-L$. Sejam $t_{1}, t_{2}$ e $t_{3}$ três terminais distintos nesse componente e seja $m$ o ancestral comum de $t_{1}, t_{2}$ e $t_{3}$ com o menor bp-número. Se $m$ é um C-nó (ativo) então sejam $t_{1}^{\prime}, t_{2}^{\prime}$ e $t_{3}^{\prime}$ os filhos reais de $m$ e ancestrais de $t_{1}, t_{2}$ e $t_{3}$, respectivamente. Neste caso existe uma obstrução $\left(C, t_{1}^{\prime}, t_{2}^{\prime}, t_{3}^{\prime}\right)$ do tipo (o2), onde $C$ é o bloco $B_{m}$.

Se $m$ é um P-nó, então seja $v$ o ancestral comum em $T$ de pelo menos dois entre $t_{1}, t_{2}$ e $t_{3}$ com o menor bp-número. Se $v$ é um C-nó então, analogamente ao que foi descrito acima, uma obstrução do tipo (o2) é encontrada. Caso contrário, sejam $K_{1}, K_{2}$ e $K_{3}$ os componentes resultantes de $M-v$ que contém, respectivamente, $t_{1}, t_{2}$ e $t_{3}$. Assim, $\left(v, K_{1}, K_{2}, K_{3}\right)$ é uma obstrução do tipo (o3).

Se nenhum dos casos tratados pelos lemas 4.2, 4.3, 4.4 e 4.5 ocorre, existe um $X Y$-caminho, conforme atesta o lema abaixo.

Lema 4.6: Se a função find_obstruction devolve false, então existe um $X Y$-caminho no componente em questão.

Demonstração: No caso de um terminal, seja $Q$ o caminho em $T$ do terminal a $w$. O $X Y$ caminho consiste numa extensão do caminho $P^{\prime}$ em $M$, do terminal ao filho de $w$ em $Q$, obtido da seguinte maneira. Cada P-nó em $Q$ está em $P^{\prime}$ e, para cada C-nó (ativo) $c$ em $Q$, seja $f$ definido a seguir. Se $c$ é um terminal, então seja $f$ um filho real de $c$ tal que $b(f)=\operatorname{bp}(w)$. Caso contrário, seja $f$ o filho real de $c$ que está em $Q$. O lado de $B_{c}$, determinado por $f$ e pelo pai de $c$, associado ao lado do $\mathrm{RBC}(c)$ que foi removido durante a compactação de $\mathrm{RBC}(c)$ pela função add_old_cnode_RBC_to_new_ $R B C$ (figuras 4.22 e 4.23) está em $P^{\prime}$. 
O caso de dois terminais é análogo.

Os cinco lemas acima mostram que a função find_obstruction funciona corretamente. Com isso, se o algoritmo atinge a linha marcada com (1) na função is_planar (figura 4.4, página 55), existe um $X Y$-caminho $P$ no componente em questão. A função set_info_for_new_cnode (seção 4.8) cria um novo C-nó $c$ para representar o componente biconexo correspondente e modifica a árvore $T$ da seguinte maneira.

Seja $\bar{P}$ o caminho complementar de $P$. Durante a construção do $\operatorname{RBC}(c)$, faz-se $w$ ser o pai de $c$ em $T$ e, para cada vértice inserido em $\operatorname{RBC}(c)$, seu pai real passa a ser $c$. Os vértices inseridos em $\operatorname{RBC}(c)$ são vértices em $\bar{P}$ cujo novo valor de $b$, após a alteração de seu pai, é superior ao $\mathrm{bp}(w)$. Ao término da construção do $\mathrm{RBC}(c)$, cada vértice de $\bar{P}$ que enxerga $L^{\prime}$ através de $V_{G} \backslash V_{B_{c}}$ tem como pai real $c$ e a propriedade (T3') é válida no final de uma iteração do algoritmo. Claramente a propriedade (T4') é satisfeira.

\subsection{Eficiência}

A análise do teste de planaridade consiste na análise do tempo consumido pela função is_planar (página 55). Para analisar essa função, primeiramente analisa-se as rotinas pre_processing, find_terminals, find_obstruction e set_info_for_new_cnode.

\section{Função pre_processing}

Esta claramente consome tempo linear, já que a bp-árvore e os valores de $h$ e $b$ podem ser calculados em tempo $O(n+m)$, por meio de uma busca em profundidade no grafo. É nessa função também que é inicializado o vetor state [ ] e algumas outras informações associadas a cada vértice, o que consome tempo $O(n)$.

\section{Função find_terminals}

A menos dos while's (1) e (2) e do forall na última linha (figura 4.10, página 62), o tempo gasto pela função é $O\left(d_{w}\right)$ devido ao forall_out_edges, onde $d_{w}$ é o grau do vértice $w$ em $G$.

O tempo gasto pelo while (2) é certamente limitado pelo tempo consumido pelo while (1), já que $S$ é declarada imediatamente antes do while (1) e nós são empilhados em $S$ apenas no while (1), um por iteração. Para determinar o tempo consumido pelo forall, note que a variável traversed_nodes, declarada no início da função find_terminals, contém, imediatamente antes do 
forall, todos os nós percorridos no while (1). Ou seja, o tempo do forall também é dominado pelo tempo do while (1).

Denote por $k_{w}$ o número de nós armazenados na variável traversed_nodes depois do forall_out_edges. O tempo total consumido pelo while (1), considerando todas as iterações do forall, é $\theta\left(k_{w}+d_{w}\right)$ pois, em cada iteração do forall_out_edges, o while (1) visita no máximo dois vértices já percorridos - um antes de sair do while (1) e outro possivelmente dentro da função find_active_cnode.

\section{Função find_obstruction}

A função find_obstruction (figuras 4.11 e 4.12, páginas 63 e 64) basicamente percorre um subconjunto dos nós percorridos pela função find_terminals. Os únicos nós percorridos por ela que não foram percorridos por find_terminals são alguns dos nós visitados pela função test_cnode_counter (figura 4.16, página 68 ). Tais nós podem ser incluídos no contador $k_{w}$ que é analisado globalmente. Resumindo, o tempo consumido pela função find_obstruction é $O\left(k_{w}\right)$, onde $k_{w}$ é o número de nós percorridos ou pela função find_terminals ou pela função find_obstruction durante a iteração em que $w$ é processado.

Função set_info_for_new_cnode

O tempo consumido por essa rotina é $O\left(k_{w}\right)$ pois ela consome tempo linear no número de nós que foram visitados anteriormente por find_terminals ou por find_obstruction.

Assim, para concluir a análise da função is_planar, resta totalizar o tempo consumido nas iterações do for da função is_planar. Isto é, resta calcular uma delimitação superior para

$$
\sum_{w=1}^{n}\left(k_{w}+d_{w}\right)=\sum_{w=1}^{n} k_{w}+2 m .
$$

Isso corresponde a dar uma delimitação superior para $\sum_{w=1}^{n} k_{w}$, ou seja, para o número total de nós percorridos pelo algoritmo.

\section{Número de nós percorridos}

Observe que apenas C-nós ativos e P-nós são percorridos na PC-árvore alterada, conforme descrito na seção 4.5. Um C-nó ativo é percorrido apenas em uma iteração: ao ser percorrido, ou encontra-se uma obstrução ou ele se torna inativo.

Similarmente um P-nó de bp-número menor que o de $w$ e que é filho de um outro P-nó em $T$ é percorrido em apenas uma iteração nessas condições: depois de ser percorrido, se nenhuma 
obstrução foi detectada, então ele passa a ser filho de um c-nó até o algoritmo terminar de executar.

Resta calcular quantas vezes um P-nó que é filho de um C-nó pode ser visitado durante o algoritmo. Observe que, para um C-nó ativo $c$ da PC-árvore alterada $T$, no início de cada iteração cada vértice do $\mathrm{RBC}(c)$ tem o valor de $b$ maior ou igual a $\mathrm{bp}(w)$.

No percurso na PC-árvore alterada $T$, para cada C-nó ativo $c$ que se torna inativo devido a contrução de um novo C-nó, os filhos reais de $c$, cujo valor de $b$ é igual ao bp $(w)$, são visitados um número constante de vezes e depois são omitidos do RBC do novo C-nó e no máximo dois filhos reais de $c$ cujo valor de $b$ é superior ao $\operatorname{bp}(w)$ são visitados (e são visitados um número constante de vezes).

Assim, totalizando o número de nós visitados pelo algoritmo durante todo o processamento é $O\left(n+n_{c}\right)$, onde $n_{c}$ é o número de C-nós criados. Como $n_{c}=O(n)$, pois no máximo $3 n-6$ C-nós são criados, então $\sum_{w=1}^{n} k_{w}=O(n)$. Juntando isso aos cálculos anteriores, conclui-se que o tempo total gasto pela função is_planar é $O(n)$. 



\section{Construção de um desenho plano}

Neste capítulo, são descritas as adaptações o teste de planaridade descrito no capítulo 4, necessárias para a obtenção de uma descrição combinatória plana do grafo, caso este seja planar e biconexo.

Como observado na seção 2.4, uma descrição combinatória plana é formada por listas de adjacências e pode ser representada por uma lista única de arcos. Esta representação é utilizada nesta implementação.

\subsection{Alterações na PC-árvore e no corpo do algoritmo}

Seja $H$ um grafo planar e $v$ um vértice de $H$. Uma descrição combinatória plana $D$ de $H$ é alinhada por $v$ se um de seus ciclos faciais que contém $v$, digamos $C:=\left\langle u_{0}, u_{1}, \ldots, u_{k-1}, u_{0}\right\rangle$, é tal que, para cada $i$, o arco $u_{i} u_{i+1}$ é o primeiro arco da lista $D\left(u_{i}\right)$, considerando-se os subíndices módulo $k$. Neste caso, $C$ é o ciclo facial determinado por $D$ e $v$ (figura 5.1).

Para obter uma descrição combinatória plana do grafo dado, o algoritmo de SH mantém, para cada C-nó ativo $c$ da PC-árvore, uma descrição combinatória plana particular do bloco em $H+w$ associado ao bloco $B_{c}$ da moldura, denotada por $\operatorname{DCP}(c)$. A $\operatorname{DCP}(c)$ é alinhada pelo pai de $c$ na PC-árvore $T$. Além disso, o ciclo facial determinado pela $\mathrm{DCP}(c)$ e pelo pai de $c$ em $T$, chamado de ciclo facial externo de $c$, contém todos os vértices do $\mathrm{RBC}(c)$. (Na verdade, o ciclo facial externo de um C-nó ativo $c$ corresponde ao bloco $B_{c}$ da moldura corrente.)

Com essa informação extra, é possível obter a moldura em cada iteração do algoritmo: basta juntar as arestas de $T-L$ cujas pontas são vértices de $G$ e os ciclos faciais externos de cada C-nó ativo de $T$.

Na implementação, para cada C-nó ativo $c$ de $T$, a $\mathrm{DCP}(c)$ é armazenada na variável global embed_list [c], que é uma lista duplamente ligada do tipo bmlist descrito na declaração de uma 


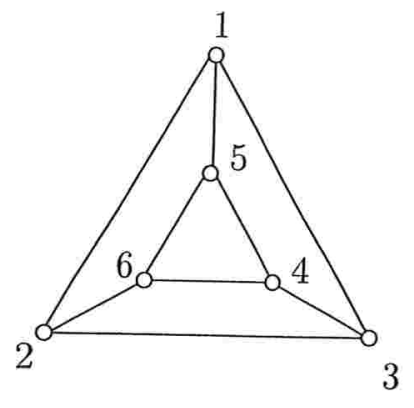

Descrição combinatória plana $D$ alinhada por 1 e por 4 : $D=\langle(6,4),(1,3),(1,5),(2,1),(2,6),(2,3),(3,2),(3,4),(3,5),(4,5)$, $(4,3),(4,6),(5,6),(5,1),(5,4),(6,2),(6,5),(1,2)\rangle$

$$
\begin{aligned}
& D(1)=\langle(1,3),(1,5),(1,2)\rangle \\
& D(2)=\langle(2,1),(2,6),(2,3)\rangle \\
& D(3)=\langle(3,2),(3,4),(3,5)\rangle \\
& D(4)=\langle(4,5),(4,3),(4,6)\rangle \\
& D(5)=\langle(5,6),(5,1),(5,4)\rangle \\
& D(6)=\langle(6,4),(6,2),(6,5)\rangle
\end{aligned}
$$

Ciclo facial determinado por $D$ e $1:\langle 1,3,2,1\rangle$

Ciclo facial determinado por $D$ e $4:\langle 4,5,6,4\rangle$

Figura 5.1: Uma descrição combinatória plana alinhada por 1 e por 4 .

PC-árvore alterada na página 53. Na última iteração, o algoritmo constrói uma lista (chamada de final_emb_list na implementação) que representa a descrição combinatória construída para $G$, caso $G$ seja planar. Finalmente, através da função sort_edges do LEDA, as listas de adjacência de $G$ são reordenadas de acordo com a ordem em final_emb_list.

Em cada iteração do algoritmo, durante a construção de um novo C-nó c, a função calculate_partial_embedding (figuras 5.2 e 5.3) é acionada pela função is_planar, no ponto marcado com (1) na figura 4.4 (página 55) para construir a $\operatorname{DCP}(c)$.

$\{$ list $<$ node $>$ traversed_nodes, list_representants;

list $<$ edge $>$ \& list_back_edges, list $<$ node $>\&$ terminal_nodes $)$

switch (terminal_nodes.size ()$)\{$

case $1:\{$

map $<$ node, node $>$ backedge_representant;

node $t e r m=$ terminal_nodes.contents $($ terminal_nodes.first ()$)$;

mark_path_in_T(term, $w$, backedge_representant, traversed_nodes);

map $<$ node, list $<$ edge $>>b_{\text {_edges_repres; }}$

$b_{-} e d g e s \_r e p r e s=g r o u p \_b a c k \_e d g e s \_b y \_r e p r\left(G, l i s t \_b a c k \_e d g e s\right.$,

list $<$ node $>L_{-} l$;

backedge_representant, traversed_nodes, list_representants);

$L_{-} l=$ embed_upward_T(term, $w, G, w, b_{-}$edges_repres, traversed_nodes, embed_list $[$new_cnode $\left.]\right)$;

Figura 5.2: Código da função calculate_partial_embedding. 

node $t$

Figura 5.3: (Continuação) Código da função calculate_partial_embedding.

\subsection{Construção da DCP de um novo C-nó}

Considere uma iteração do algoritmo. Lembre-se de que $w$ denota o vértice examinado nesta iteração e $K$ é o componente em $T-L$ que está sendo processado neste instante.

A construção da DCP consiste em decidir a ordem cíclica em torno de $w$ das arestas de retorno com uma ponta em $w$ e outra em $K$. (Esta ordem induz a ordem das demais arestas em $K$.) A construção da $\mathrm{DCP}(c)$ para o caso em que há apenas um terminal é descrita a seguir. $\mathrm{O}$ caso de dois terminais é similar, mas exige mais detalhes técnicos, por isso será omitido.

Grosseiramente, a construção da $\operatorname{DCP}(c)$ pode ser descrita da seguinte maneira. Seja $P^{\prime}$ o subcaminho do $X Y$-caminho $P$ do terminal $t$ ao filho de $w$ (em $T$ ) em questão. Cada aresta de retorno em questão tem uma ponta em alguma subárvore enraizada num nó de $P^{\prime}$, conforme a figura 5.4(a). O primeiro passo da construção da $\operatorname{DCP}(c)$ consiste na separação dessas arestas de retorno em grupos dados pela raiz em $P^{\prime}$ da subárvore em questão. O segundo passo é a construção da $\operatorname{DCP}(c)$ para cada um desses grupos.

Na verdade, devido à assimetria introduzida pela orientação da PC-árvore $T$, algumas arestas de retorno são associadas a vértices que não estão em $P^{\prime}$. Mais precisamente, algumas arestas são associadas a P-nós (ou a filhos reais dos C-nós) que estão no caminho de $p$ a $w$ em $T$ da figura 5.4(b). Tal situação ocorre quando o $X Y$-caminho $P$ não termina por uma aresta que está em $T$. As arestas de retorno associadas a vértices fora de $P^{\prime}$ devem aparecer antes das 


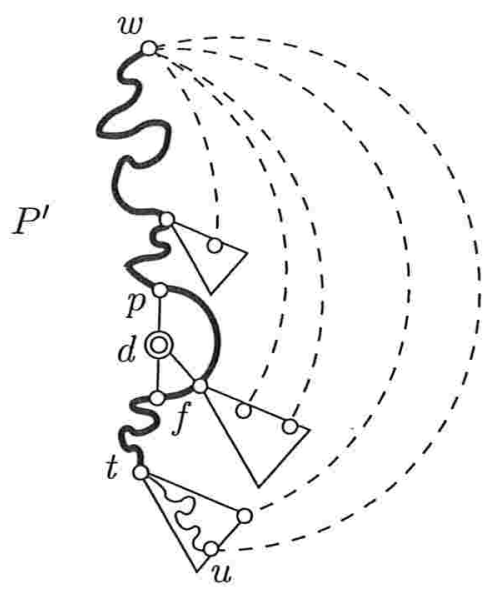

(a)

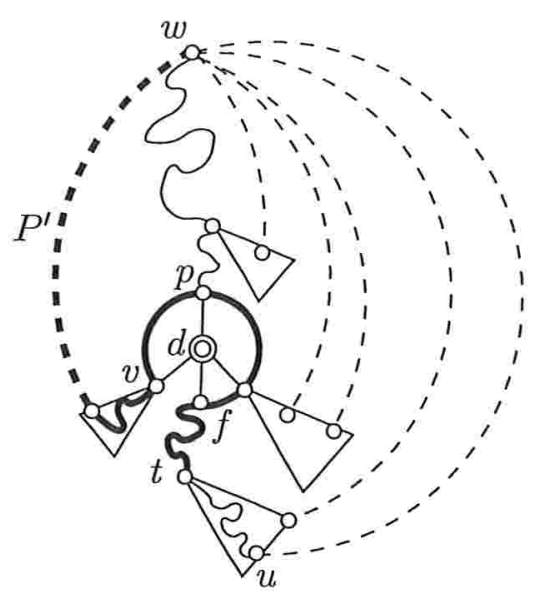

(b)

Figura 5.4: Em (a) e (b), o subcaminho $P^{\prime}$ de um $X Y$-caminho com um único terminal $t$ é indicado por linhas grossas e as arestas de retorno por linhas tracejadas.

arestas de retorno associadas a vértices que estão na parte final de $P^{\prime}$, após o pai do último C-nó atravessado por $P^{\prime}-$ o vértice $p$ da figura 5.4(b). Neste caso, a função embed_upward_T (descrita mais adiante) devolve uma lista de vértices correspondente a parte final de $P^{\prime}$ (e os vértices à "esquerda" no RBC dos C-nós ativos no caminho de $p$ a $w$ em $T$ da figura 5.4(b)) e tais vértices são processados após a chamada da função. (Esse processamento é feito fora da função embed_upward_T para que esta possa ser usada também no caso de dois terminais.)

Abaixo encontra-se uma descrição da função calculate_partial_embedding (figuras 5.2 e 5.3), bem como das funções invocadas por ela.

O primeiro passo da construção da $\operatorname{DCP}(c)$ consiste na separação das arestas de retorno em questão em grupos. A idéia é que a ordem relativa entre arestas em grupos distintos é determinada pela ordem em que as raízes das subárvores correspondentes aparecem. Esta ordenação, juntamente com a ordenação das arestas num mesmo grupo, induz uma ordem cíclica das arestas de retorno em torno de $w$ e das demais arestas em $K$.

Seja $Q$ o caminho de $t$ a $w$ em $T$. Uma aresta de retorno $f=w z$ é representada pelo vértice $z$. Assim, na verdade, associa-se vértices a vértices. Inicialmente o vértice $z$, representando $f$, é associado ao nó $v$ de $Q$ se existe caminho internamente disjunto de $Q$ de $z$ a $v$. Se $v$ é um C-nó, então $z$ é associado ao filho real de $v$ que está no caminho de $z$ a $v$ em $T$. Isso é feito da seguinte maneira. Primeiramente marca-se apenas os nós do caminho $Q$ como visitados e cada um desses nós é associado a si próprio, através da variável backedge_representant. Isso é feito pela rotina mark_path_in_T (figura 5.5). 


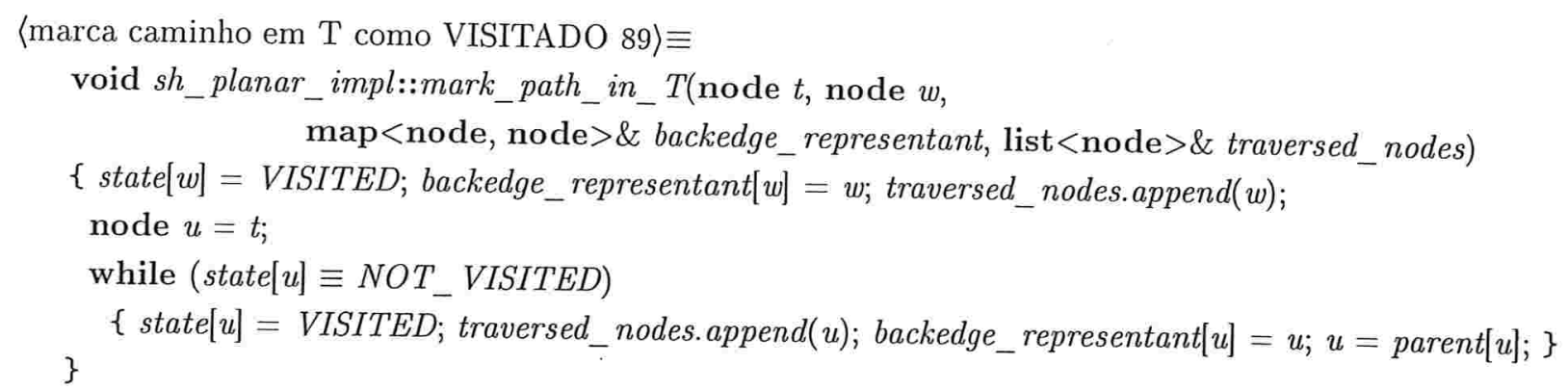

Figura 5.5: Código da função mark_path_in_T.

Para cada aresta $f=w z$, tal que $\operatorname{bp}(z)<\mathrm{bp}(w)$, sobe-se em $T$ a partir de $z$ até chegar-se a um nó visitado $v$. Se $v$ está associado a um C-nó (neste caso, note que $v$ está associado a si próprio), então $z$ é associado ao filho real de $v$ que está no caminho de $z$ a $v$ em $T$. Caso contrário, seja $u$ o vértice ao qual $v$ está associado. Neste caso, $z$ é associado a $u$. Durante este processo, marca-se todos os nós de $z$ até $v$ como visitados, associando-os ao mesmo vértice ao qual $z$ foi associado. Terminado este processo, cada aresta de retorno em questão (na verdade, a sua ponta mais baixa em $T$ ) está associada a um P-nó de $Q$ ou a um filho real de um C-nó de $Q$. O código correspondente a este processo é dado pela função group_back_edges_by_repr (figuras 5.6 e 5.7).

O segundo passo é a construção da $\operatorname{DCP}(c)$ para cada um dos grupos criados no passo anterior. Este passo é efetuado pela função embed_upward_T (figura 5.8), que é descrita a seguir.

Inicialmente, a lista $\operatorname{DCP}(c)$ é vazia. Para cada nó $v$ de $Q$, subindo em $T$ a partir de $t$, se $v$ é um C-nó ativo, então a função add_old_cnode_to_embedding (figura 5.13) é chamada para incluir a $\operatorname{DCP}(v)$, de maneira conveniente, no início da $\operatorname{DCP}(c)$. Se $v$ é um P-nó e tem um filho em $Q$, então seja $v^{\prime}$ esse filho. Se $v^{\prime}$ é um P-nó, os pares $v v^{\prime}$ e $v^{\prime} v$ são adicionados no início da $\operatorname{DCP}(c)$. Em seguida, a função embed_back_edges (figuras 5.9 e 5.10) é chamada para incluir à $\mathrm{DCP}(c)$ os pares referentes à subárvore de $T$ enraizada em $v$, denotada por $T_{v}^{*}$, definida a seguir. A árvore $T_{v}^{*}$ coincide com $T_{v}$ se $v=t$ e, caso contrário, $T_{v}^{*}:=T_{v}-T_{v^{\prime}}$, onde $v^{\prime}$ é o filho de $v$ em $Q$.

Para cada C-nó ativo $c$ em $Q$, os vértices à "esquerda" no $\mathrm{RBC}(c)$ são inseridos na lista to_embed_later, pela função add_old_cnode_to_embedding, explicada mais adiante. Essa lista contém tais vértices na ordem em que as arestas de retorno associadas aparecem em torno de $w$, e é devolvida ao final da função embed_back_edges. 
90a 〈group back-edges 90a) $\equiv$

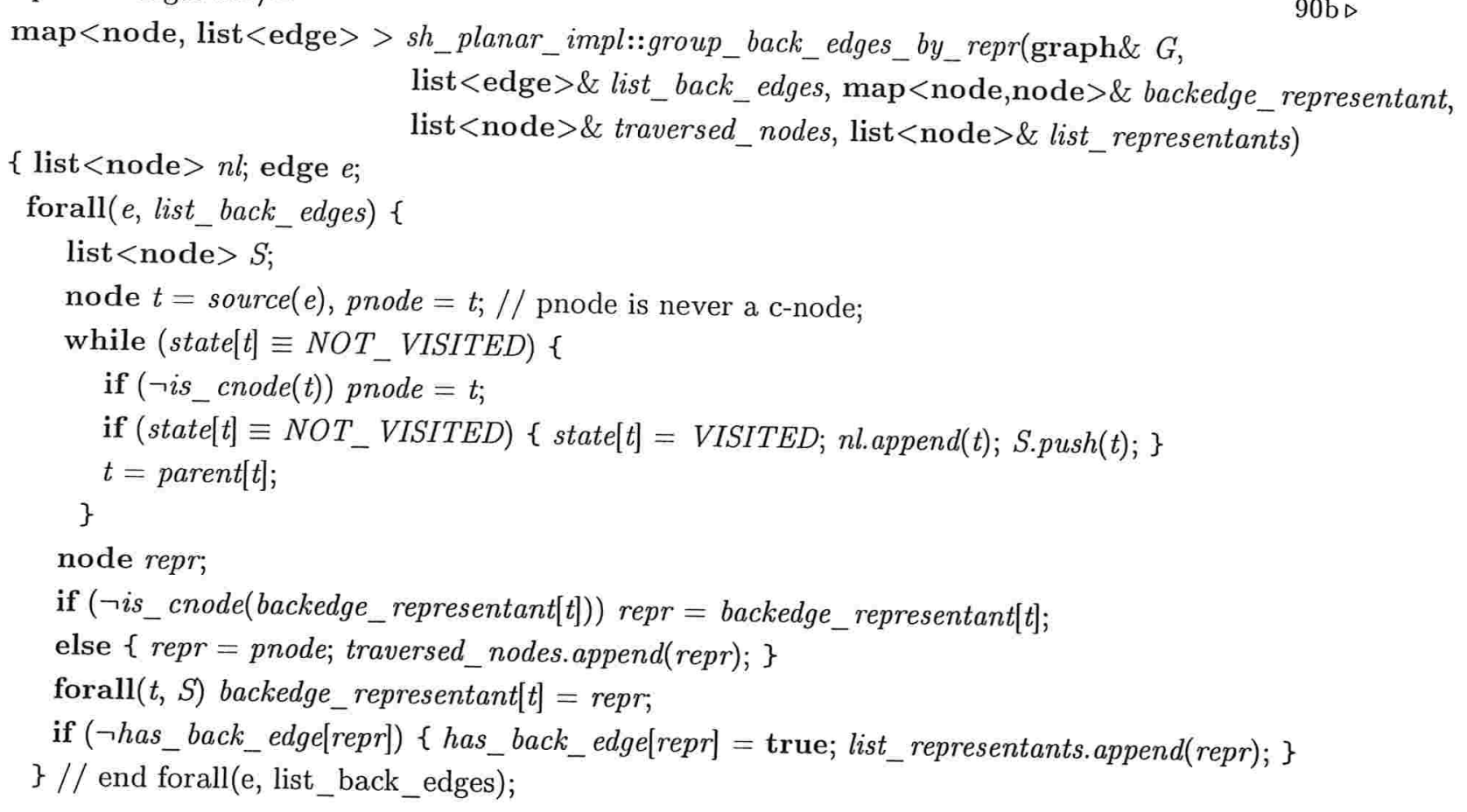

Figura 5.6: Código da função group_back_edges_by_repr.

90b 〈group back-edges 90a $\rangle+\equiv$

map $<$ node, list<edge $>>$ list_edges;

$\triangleleft 90 \mathrm{a}$

forall $(e$, list_back_edges $)\{$ node $u=\operatorname{source}(e) ;$ list_edges[backedge_representant $[u]]$. append $(e) ;\}$ node $t$;

forall $(t, n l)$ state $[t]=N O T_{-}$VISITED;

forall $(t$, list_representants $)$ state $[t]=V I S I T E D$;

return list_edges;

\}

Figura 5.7: (Continuação) Código da função group_back_edges_by_repr. 
91 〈cálculo da DCP subindo em T 91) 三

list $<$ node $>s h \_p l a n a r \_i m p l:: e m b e d \_u p w a r d \_T($ node $t$, node $u$,graph $\& G$, node $w$,

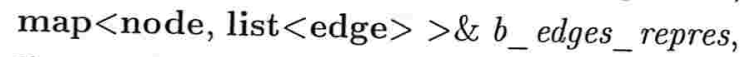

$\{$ list $<$ node $>$ to_embed_later; list $<$ node $>\&$ traversed_nodes, bmlist $<$ edge $>\&$ emb_list)

node $v=t$, pred_ $v=\mathbf{n i l}$;

while $($ pred_v $v \neq u)\{$

if (is_cnode $(v))$ \{

node $f=$ pred_ $v$, oldcnode $=v$;

add_old_cnode_to_embedding $\left(G, w\right.$, oldcnode, $f, b_{-}$edges_repres, traversed_nodes,

$v=$ parent $[$ oldcnode $]$ to_embed_later, emb_list);

if $(v \equiv u)$ return to_embed_later;

\}

else if ( $p r e d \_v \neq$ nil $)\{$

emb_list.push(G.reverse $\left(T 0 \_e d g e_{-}\right.$in $[$pred_v $\left.\left.]\right)\right)$;

if $(v \neq w)$ emb_list.push $\left(T 0_{-} e d g e_{-} i n[\right.$ pred_v]);

else $e m b \_l i s t . a p p e n d\left(T 0 \_e d g e_{-} i n\left[p r e d \_v\right]\right)$;

if (has_back_edge $[v] \wedge v \neq u$ )

embed_back_edges $\left(G, v\right.$, traversed_nodes, $b_{-}$edges_repres $[v]$, emb_list $)$;

pred_v $=v ; v=$ parent $[v]$;

\} // while;

return to_embed_later;

\}

Figura 5.8: Código da função embed_upward_T. 
função embed_back_edges

A função embed_back_edges (figuras 5.9 e 5.10) constrói, para um dado vértice $v$, duas listas de pares de vértices, $L_{T}(v)$ e $L_{w}(v)$, que são concatenadas no início e no fim da $\mathrm{DCP}(c)$ corrente, respectivamente.

Figura 5.9: Código da função embed_back_edges.

Inicialmente, as arestas de retorno associadas a $v$ são ordenadas por uma bp-ordenação de $T_{v}^{*}$, respeitando a ordem fixada para cada C-nó ativo em $T_{v}^{*}$. Esse processo é realizado pela função sort_back_edges_by_dfs (figura 5.11). Essa função começa calculando uma subárvore $J$ de $T_{v}^{*}$ onde, para cada aresta de retorno $w z$ associada a $v, J$ é a união dos caminhos de $z$ a $v$ em $T$.

A lista de adjacências de cada C-nó ativo $d$ da árvore $J$ é reorganizada de maneira a preservar a ordem dos filhos reais de $d$ em $T$ (lembre-se que a atualização de $T$ ainda não foi executada nesta iteração). A função termina fazendo uma busca em profundidade na árvore $J$, obtendo uma lista $R_{v}$ das arestas de retorno $w z$ associadas a $v$, ordenada pelo bp-número de $z$. (Essa lista é representada pela variável back_edge/ / nas figuras 5.9 e 5.10.)

Após a ordenação das arestas associadas a um vértice $v$, prossegue-se à construção da $\mathrm{DCP}(c)$ pela função embed_back_edges. Nessa função, marca-se todos os vértices de $J$ como nãovisitados, exceto $v$ que é marcado como visitado. As listas $L_{T}(v)$ e $L_{w}(v)$ começam vazias. Para cada aresta $w z$ na lista $R_{v}$, inclui-se no fim de $L_{w}(v)$ o arco $w z$.

Para cada aresta $w z$ na lista $R_{v}$, em ordem reversa, inicia-se uma lista auxiliar el com o $\operatorname{arco} z w$ e percorre-se a árvore $J$, a partir de $z$, subindo até o primeiro nó visitado. Cada aresta $x y$ percorrida nesse percurso acarreta a inclusão, no início da lista $e l$, dos arcos $x y$ e $y x$. Ao final deste percurso, a lista el é concatenada ao fim da lista $L_{T}(v)$ corrente e passa-se à próxima aresta 


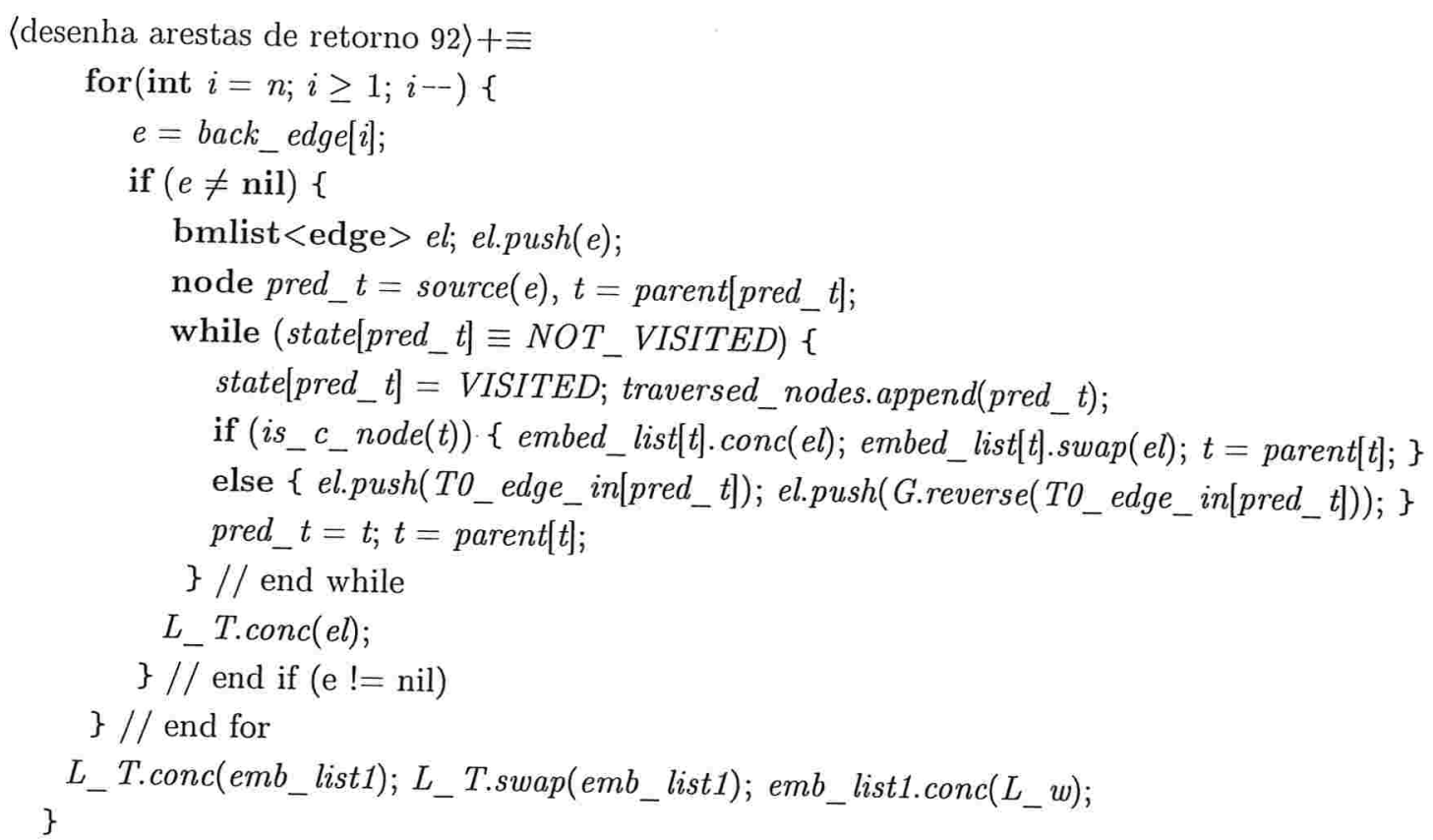

Figura 5.10: (Continuação) Código da função embed_back_edges.

de retorno de $R_{v}$. Isso completa a descrição da função embed_back_edges. Uma ilustração do que é feito por esta função se encontra na figura 5.12 .

função add_old_cnode_to_embedding

Seja $v$ um C-nó ativo e sejam $f$ o filho real de $v$ que está no $X Y$-caminho $P$ e $p$ o pai de $v$. Seja $L_{l}$ o conjunto dos vértices em $\mathrm{RBC}(v)$ entre $f$ e $p$, à esquerda (cyclic_pred) de $p$, e seja $L_{r}$ o conjunto dos vértices em $\mathrm{RBC}(v)$ entre $f$ e $p$, à direita (cyclic_succ) de $p$. Se algum vértice de $L_{r}$ enxerga $Y$ através de $\left(V_{G} \backslash L\right) \backslash V_{B_{v}}$, então a $\operatorname{DCP}(v)$ é invertida e as listas $L_{l}$ e $L_{r}$ são trocadas entre si (deste modo, nenhum vértice em $L_{r}$ enxerga $Y$ através de $\left.\left(V_{G} \backslash L\right) \backslash V_{B_{v}}\right)$. Para cada vértice $u$ em $L_{r}$ que enxerga $X$ através de $\left(V_{G} \backslash L\right) \backslash V_{B_{v}}$, na ordem dada pelo RBC $(v)$, a função embed_back_edges é chamada para incluir os arcos devido a subárvore $T_{u}$ à $\operatorname{DCP}(v)$. Em seguida, concatena-se $\operatorname{DCP}(v)$ no início da lista $\operatorname{DCP}(c)$ e concatena-se $L_{l}$ no início da lista emblater, que contém os vértices a serem incluídos à $\mathrm{DCP}(c)$ no final da execução da função calculate_partial_embedding (figura 5.3).

Para encerrar a construção de uma descrição combinatória plana de um grafo planar, resta apenas inserir os arcos à descrição que têm uma ponta na raiz $r$ de $T$. Isto é feito pela função embed_root, chamada no ponto marcado com (2) na função is_planar (figura 4.4, página 55). Neste caso, cada aresta de retorno $r z$ é associada a $r$. Seja $J$ a união dos caminhos de $z$ a $r$ 
〈ordena arestas de retorno 94 〉三

int $s h_{-} p l a n a r_{-} i m p l:: s o r t_{-} b a c k_{-} e d g e s_{-} b y_{-} d f\left(\right.$ node $w$, node $r e p r$, list $<$ edge $>\& l i s t_{-} b a c k \_e d g e s$,

$\{\operatorname{graph} D$;

array $<$ edge $>\&$ back_edge)

list $<$ node $>$ list_nodes, list_cnodes;

map $<$ node, node $>$ node_in_ $D$, node_in_G;

edge $e$;

node_in_D $[$ repr $]=$ D.new_node();

node_in_G[node_in_D $D[$ repr $]]=r e p r$;

forall $(e$, list_back_edges $)\{$

node $t=\operatorname{source}(e)$, pred_t $t=$ nil;

while $\left(\right.$ state $[t] \equiv N O T_{-}$VISITED $)\{$

if $\left(i s \_\right.$cnode $\left.(t)\right)$ list_cnodes.append $(t)$;

state $[t]=V I S I T E D ;$ list_nodes.append $(t)$;

node_in_ $D[t]=D . n e w_{-}$node () ;

node_in_G[node_in_ $D[t]]=t$;

if ( red_t $t \neq$ nil) D.new_edge(node_in_ $D[t]$, node_in_ $D[$ pred_t $t])$;

pred_t $t=t ; t=$ parent $[t]$

\} // while

if $($ pred_t $t \neq$ nil) D.new_edge $($ node_in_ $D[t]$, node_in_ $D[$ pred_t $])$;

\} // forall

node $u$;

forall $(u$, list_nodes $)$ state $[u]=N O T_{-}$VISITED;

$\operatorname{map}<$ node, bool $>$ is_in_ $D$;

forall( $u$, list_cnodes $)\{$

node $t$;

$\operatorname{myforall}(t, R B C 1[u])$ is_in_D $[t]=$ false;

list<edge $>e l$;

forall_out_edges $(e$, node_in_ $D[u])\left\{i s_{-} i n_{-} D[\right.$ node_in_G $[\operatorname{target}(e)]]=\operatorname{true} ;$ el.append $\left.(e) ;\right\}$

D.del_edges $(e l)$;

myforall_rev $(t, R B C 1[u])$

\}

if $\left(i s_{-} i n_{-} D[t]\right)$ D.new_edge(node_in_ $D[u]$, node_in_ $\left.D[t]\right)$;

node_array<int $>d f \operatorname{spos}(D, 0)$;

$D F S_{-} P O S(D$, dfspos $)$;

int tot $=$ D.number_of_nodes () ;

back_edge.resize $(1$, tot $)$;

for (int $i=1 ; i \leq$ tot; $i+$ ) back_edge[i] = nil;

forall $(e$, list_back_edges $)\left\{\right.$ node $u=\operatorname{source}(e) ; b a c k \_$edge $[$dfspos $[$node_in_D $D[u]]=e ;\}$ return tot;

\}

Figura 5.11: Código da função sort_back_edges_by_dfs. 


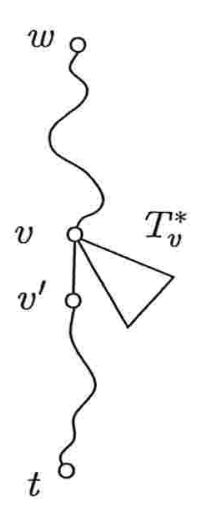

(a)

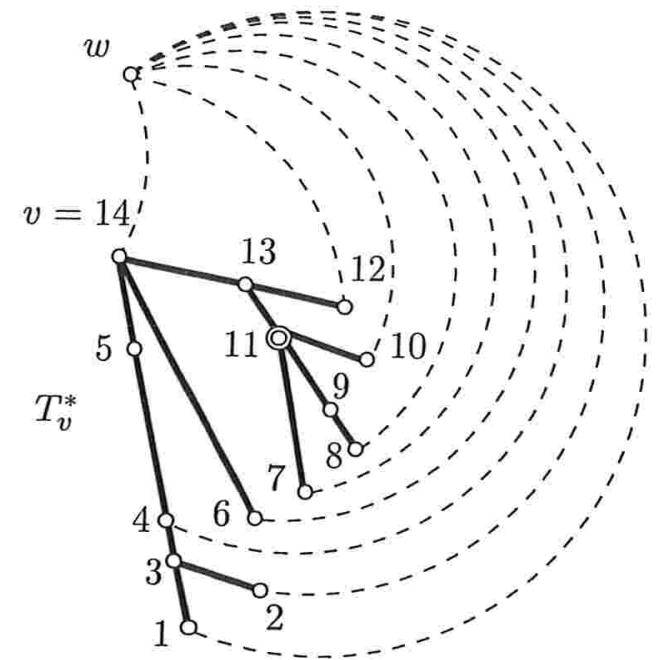

(b)

$\mathrm{DCP}(c)^{\prime}$ :

$\langle(14, w),(14,13),(13,14),(13,12),(12, w),[\mathrm{DCP}(11)],(10, w),(9,8),(8,9),(8, w)$, $(7, w),(14,6),(6,14),(6, w),(14,5),(5,14),(5,4),(4,5),(4, w),(4,3),(3,4)(3,2),(2,3),(2, w)$ $(3,1),(1,3),(1, w),\left(14, v^{\prime}\right),\left(v^{\prime}, 14\right), \mathrm{DCP}(c)$, $(w, 1),(w, 2),(w, 4),(w, 6),(w, 7),(w, 8),(w, 10),(w, 12),(w, 14)\rangle$

(c)

Figura 5.12: (a) Dado um vértice $v$, para construir a DCP de num novo C-nó, é necessário decidir a ordem cíclica das arestas incidentes em $v$, bem como as arestas contidas em cada subárvore $T_{v}^{*}$ (ilustradas por triângulos). (b) Construção da $\operatorname{DCP}(c)$ em $T_{v}^{*}$ (função embed_back_edges). As arestas de retorno estão em linhas tracejadas. (c) $\mathrm{DCP}(c)$ após processar $v$. 
(inclui DCP de um c-nó antigo a DCP de um novo c-nó 96)三 void $s h \_p l a n a r_{-} i m p l:: a d d_{-}$old_cnode_to_embedding(graph\& $G$, node $w$, node oldcnode, node $u$, map $<$ node, list $<$ edge $>>\&$ b_edges_repres, list $<$ node $>\&$ traversed_nodes,

$\{$ bmlist_item $i t=R B C 1[$ oldcnode].first_item( $)$, list $<$ node $>$ \& emblater, bmlist $<$ edge $>$ \& emb_list 1 )

$i t l=R B C 1[$ oldcnode $] . c y c l i c \_p r e d(i t, \mathbf{n i l}), i t r=R B C 1[$ oldcnode $] . c y c l i c \_s u c c(i t, \mathbf{n i l}) ;$

node $j l=R B C 1[$ oldcnode].contents $(i t), j r=R B C 1[$ oldcnode].contents $(i$ itr $)$;

list $<$ node $>L_{-} l, L_{-} r$;

bmlist_item aux, $s=i t$;

while (label_b $\left.[j l] \leq d f s p o s_{-} n u m[w]\right)\{$

if $\left(\right.$ label_b $\left.[j l] \equiv d f s p o s \_n u m[w]\right) L_{-}$l.append $(j l)$;

\}

$a u x=i t l ; i t l=R B C 1[$ oldcnode $] . c y c l i c \_p r e d(i t l, s) ; s=a u x ; j l=R B C 1[$ oldcnode].contents(itl);

bmlist_item $p=i t$;

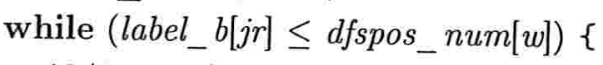

if (label_b $\left.[j r] \equiv d f s p o s \_n u m[w]\right) L_{-} r$ rappend $(j r)$;

\}

$a u x=i t r ; i t r=R B C 1[$ oldcnode].cyclic_succ(itr, $p) ; p=a u x ; j r=R B C 1[$ oldcnode].contents(itr);

$/ / \mathrm{u}==$ nil when oldcnode is a terminal node;

bool flipped $=\left(\left(L_{-}\right.\right.$l.size ()$>0 \wedge(j l \equiv u \vee u \equiv$ nil $\left.\left.)\right) \vee(j r \neq u \wedge u \neq \mathbf{n i l})\right)$;

if (flipped) $L_{-}$l.swap $\left(L_{-} r\right)$;

$L_{-}$r.reverse_items () ;

node $t$;

forall $\left(t, L_{-} r\right)$ embed_back_edges $\left(G, t, t\right.$ traversed_nodes, $b_{-}$edges_repres $[t]$, emb_list 1$)$;

if (fipped) embed_list[oldcnode].reverse();

embed_list[oldcnode].conc(emb_list1); embed_list[oldcnode].swap(emb_list1);

emblater.conc(L_l, LEDA::before);

\}

Figura 5.13: Código da função add_old_cnode_to_embedding. 
em T. A função embed_root inclui os arcos restantes devido a $r$ à descrição combinatória plana do grafo de acordo com uma bp-árvore em $J$, respeitando a ordem dos filhos reais para cada C-nó ativo em $J$ (o código da função embed_root é omitido). Na próxima seção, encontra-se a correção e a análise do tempo gasto para esta parte do algoritmo de SH.

As DCP's correspondentes a cada C-nó criado durante a simulação da PC-árvore alterada nas figuras 4.25 e 4.26 (páginas 77 e 78) são ilustradas na figura 5.14. A descrição combinatória plana calculada pela implementação de SH para o grafo na figura 4.1(a) se encontra na figura 5.15.

(b) $\mathrm{DCP}(-1)$ :

$\langle(2,3),(2,1),(1,2),(1,3),(3,1),(3,2)\rangle$

$\operatorname{DCP}(-3)$ :

(d) $\langle(7,8),(7,5),(5,7),(4,5),(4,3),(3,4),(3,2)$,

$(3,1),(1,3),(1,2),(2,1),(2,3),(2,5),(5,2)$,

$(5,4),(1,8),(8,1),(8,7)\rangle$

DCP $(-5)$ :

$\langle(11,12),(10,11),(10,9),(9,10),(9,11),(11,9)$,

(f) $(11,10),(9,8),(8,9),(7,8),(7,5),(5,7)$,

$(4,5),(4,3),(3,4),(3,2),(3,1),(1,3),(1,2)$,

$(2,1),(2,3),(2,5),(5,2),(5,4),(1,8),(8,1)$,

$(8,7),(1,12),(12,1),(12,11)\rangle$

DCP $(-7)$ :

$\langle(13,14),(12,13),(12,11),(12,1),(1,12),(8,7)$,

(h) $(8,1),(1,8),(5,4),(5,2),(2,5),(2,3),(2,1),(1,2)$,

$(1,3),(3,1),(3,2),(3,4),(4,3),(4,5),(5,7)$,

$(7,5),(7,8),(8,9),(9,8),(11,10),(11,9),(9,11)$,

$(9,10),(10,9),(10,11),(11,12),(10,13),(13,10)$,

$(13,12),(7,6),(6,7),(6,14),(14,6),(14,13))$
$\operatorname{DCP}(-2)$ :

(c) $\langle(4,5),(4,3),(3,4),(3,2),(3,1),(1,3)$, $(1,2),(2,1),(2,3),(2,5),(5,2),(5,4)\rangle$

(e) $\mathrm{DCP}(-4)$ :

$\langle(10,11),(10,9),(9,10),(9,11),(11,9),(11,10)\rangle$

$\mathrm{DCP}(-6)$ :

$\langle(12,13),(12,11),(12,1),(1,12),(8,7),(8,1)$,

(g) $(1,8),(5,4),(5,2),(2,5),(2,3),(2,1),(1,2),(1,3)$,

$(3,1),(3,2),(3,4),(4,3),(4,5),(5,7),(7,5)$,

$(7,8),(8,9),(9,8),(11,10),(11,9),(9,11),(9,10)$,

$(10,9),(10,11),(11,12),(10,13),(13,10),(13,12)\rangle$

$\mathrm{DCP}(-8)$ :

( $(14,15),(13,14),(12,13),(12,11),(12,1),(1,12)$,

$(8,7),(8,1),(1,8),(5,4),(5,2),(2,5),(2,3),(2,1)$,

(i) $(1,2),(1,3),(3,1),(3,2),(3,4),(4,3),(4,5)$,

$(5,7),(7,5),(7,8),(8,9),(9,8),(11,10),(11,9)$,

$(9,11),(9,10),(10,9),(10,11),(11,12),(10,13)$,

$(13,10),(13,12),(7,6),(6,7),(6,14),(14,6)$,

$(14,13),(6,15),(15,6),(15,14)\rangle$

Figura 5.14: DCP's correspondentes aos itens (b) a (i) da simulação da PC-árvore alterada nas figuras 4.25 e 4.26 (páginas 77 e 78). 


\begin{tabular}{|cl|}
\hline $1:$ & $12,8,2,3$ \\
\hline $2:$ & $5,3,1$ \\
\hline $3:$ & $1,2,4$ \\
\hline $4:$ & $3,5,16$ \\
\hline $5:$ & $4,2,7$ \\
\hline $6:$ & $7,14,15$ \\
\hline $7:$ & $5,8,6$ \\
\hline $8:$ & $7,1,9$ \\
\hline $9:$ & $8,11,10$ \\
\hline $10:$ & $9,11,13$ \\
\hline $11:$ & $10,9,12$ \\
\hline $12:$ & $13,11,1$ \\
\hline $13:$ & $14,10,12$ \\
\hline $14:$ & $15,6,13$ \\
\hline $15:$ & $6,14,16$ \\
\hline $16:$ & 15,4 \\
\hline
\end{tabular}

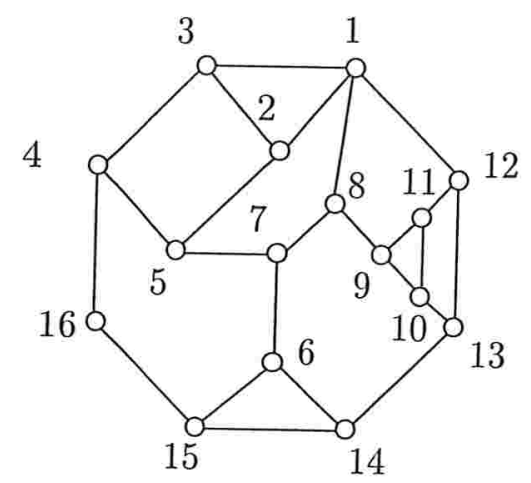

Figura 5.15: Descrição combinatória plana calculada pela implementação para o grafo na figura 4.1(a).

\subsection{Correção}

Seja $c$ o C-nó criado para representar o bloco $B$ em $H+w$ associado ao componente em $T-L$ correntemente examinado pelo algoritmo. Para mostrar que a construção de uma descrição combinatória plana descrita neste capítulo é correta, basta mostrar que:

(1) $\mathrm{DCP}(c)$ é uma descrição combinatória plana de $B$;

(2) todos os vértices de $B$ que enxergam $Y$ através de $\left(V_{G} \backslash L\right) \backslash V_{B}$ estão no ciclo facial externo de $c$.

A seguir, mostra-se que os invariantes acima valem após a construção da DCP de um novo C-nó $c$ para o caso de um terminal no componente corrente de $T-L$. (O caso de dois terminais é análogo e foi omitido.)

Seja $Q$ o caminho em $T$ do terminal a $w$. Para cada nó $v$ em $Q$, o algoritmo acrescenta a $\operatorname{DCP}(c)$ os arcos devidos a $v$ da seguinte maneira.

Se $v$ é um P-nó que não é terminal, então sejam $v^{\prime}$ o filho de $v$ em $Q$ e $p$ o pai de $v$ em $T$. A construção de $\operatorname{DCP}(c)$ é feita de tal forma que o arco $v p$, os $\operatorname{arcos}$ devidos a $T_{v}-T_{v^{\prime}}$ e o arco $v v^{\prime}$ aparecem nesta ordem em DCP(c) (figura 5.16). Assim, os únicos vértices desta parte 
que possivelmente enxergam $Y$ através de $\left(V_{G} \backslash L\right) \backslash V_{B}$ são $p, v$ e $v^{\prime}$, que estão no ciclo facial externo de $c$. Lembrando que os arcos devidos a $T_{v}-T_{v^{\prime}}$ são inseridos de acordo com uma bp-árvore, respeitando a ordem dos filhos reais dos C-nós ativos em questão, e que cada arco com ponta inicial em $w$ é inserido em $\mathrm{DCP}(c)$ refletindo a ordem de inserção do seu reverso, podemos concluir que a lista $\operatorname{DCP}(c)$ é uma descrição combinatória plana.

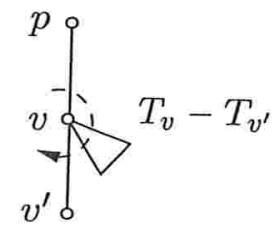

Figura 5.16: Ordem dos arcos em torno de um P-nó $v$ que não é terminal.

Se $v$ é um P-nó e um terminal, então os $\operatorname{arcos} v p$ e aqueles devido a $T_{v}$ são inseridos nesta ordem em $\mathrm{DCP}(c)$. Analogamente ao caso anterior, os vértices $v$ e $p$ estão no ciclo facial externo de $c$ (figura 5.17) e $\operatorname{DCP}(c)$ é uma descrição combinatória plana.

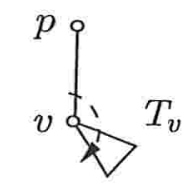

Figura 5.17: Ordem dos arcos em torno de um P-nó $v$ que é terminal.

Se $v$ é um C-nó ativo que não é um terminal, então sejam $p$ o pai de $v$ e $v^{\prime}$ o filho real de $v$ em $Q$. Seja $Y_{v}$ o conjunto dos vértices de $B_{v}$ que enxergam $Y$ através de $\left(V_{G} \backslash L\right) \backslash V_{B_{v}}$. Durante a construção da nova descrição, a $\operatorname{DCP}(v)$ é invertida, se necessário, de forma que os vértices em $Y_{v}$ estejam no lado esquerdo, determinado por $p$ e $v^{\prime}$, no ciclo facial externo de $v$ (figura 5.18). Assim, após concatenar $\operatorname{DCP}(v)$ no início de $\operatorname{DCP}(c)$, os vértices em $Y_{v}$ estão no ciclo facial externo de $c$. Observe que o arco $p p^{\prime}$, os arcos em $\operatorname{DCP}(v)$ e os arco $v^{\prime} u^{\prime}$ são inseridos nesta ordem à $\operatorname{DCP}(c)$ e que, para cada vértice $u$ no lado direito, determinado por $p$ e $v^{\prime}$, no ciclo facial externo de $v$, basta concatenar a lista dos arcos devidos a $T_{u}$ à $\mathrm{DCP}(c)$.

Se $v$ é um C-nó ativo que é terminal, então $v^{\prime}$ é o filho real de $v$ que enxerga somente $X$ através de $\left(V_{G} \backslash L\right) \backslash V_{B_{v}}$ e é adjacente a um vértice de $Y_{v}$ no $\operatorname{RBC}(v)$. Este caso é análogo ao caso anterior e isto conclui a correção para o caso de um terminal. 


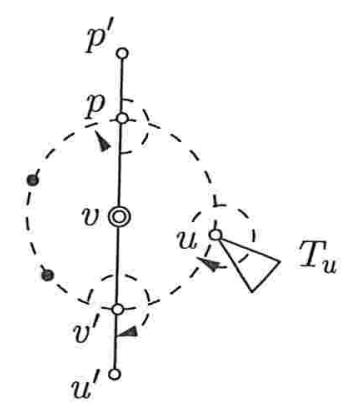

Figura 5.18: Arcos devidos a um C-nó ativo $v$. Os vértices em $\operatorname{RBC}(v)$ que enxergam $Y$ através de $\left(V_{G} \backslash L\right) \backslash V_{B_{v}}$ estão à esquerda de $v$ e estão ilustrados por círculos pretos.

\subsection{Eficiência}

Seja $w z$ uma aresta de retorno e seja $J$ a união dos caminhos de $z$ a $w$ em $T$. O tempo consumido pela função calculate_partial_embedding é proporcional ao tempo gasto para percorrer $J$ mais o tempo gasto para inserir a DCP de cada C-nó ativo no componente à nova DCP pela função add_old_cnode_to_embedding.

A função $a d d_{-}$old_cnode_to_embedding é similar à função test_cnode_counter analisada no capítulo anterior, e o tempo gasto para percorrer $J$ é limitado superiormente pelo tempo gasto durante a fase de busca por uma $X Y$-obstrução. A análise para o número de nós percorridos também é análoga ao que foi feito no capítulo anterior e, portanto, o algoritmo de SH constrói uma descrição plana de um grafo planar em tempo $O(n)$. 


\section{Obstruções}

Neste capítulo, são descritas as adaptações do teste de planaridade descrito no capítulo 4 necessárias para a obtenção de uma lista de arcos de uma obstrução, caso o grafo não seja planar. Abaixo, encontra-se uma descrição de cada uma das funções usadas para este cálculo.

\subsection{Informações adicionais para o cálculo da lista de arcos}

Para o cálculo da lista de arcos de uma obstrução, são necessárias algumas informações adicionais. Durante o algoritmo de SH, para cada vértice $v$ de $G$, a variável global node_label_b[v] é um apontador para um vértice que é descendente de $v$ em $T$ cujo valor de $h$ é igual a $b(v)$, e para cada terminal $t$, a variável global neighbor_w_terminal[ $t]$ é um apontador para um vizinho de $w$ que é descendente de $t$ em $T$. Durante o pré-processamento, guarda-se $T_{0}$ através da variável global $p 0$ [ ], que é um apontador para o pai em $T_{0}$ definido para cada vértice de $G$. Se $r$ é a raiz de $T_{0}$ então $p 0[r]$ é nil. Além disso, para cada nó $v$ em $T_{0}$, a variável global To_edge_in $[v]$ guarda um apontador para o arco com ponta inicial no pai de $v$ em $T_{0}$ e ponta final em $v$.

\subsection{Obstrução para o teste do contador de um C-nó}

A função obstruction_edges_cnode_counter (figura 6.1) é acionada quando a função test_cnode_counter, descrita na seção 4.7, detecta uma obstrução do tipo (o1) durante o teste de um C-nó ativo c. Ela é chamada pela função test_obstruction_from_terminal_node (figura 4.13, página 65) e pela função find_obstruction (figuras 4.11 e 4.12 , páginas 63 e 64 ) nos pontos marcados com (1).

Seja $p$ o pai de $c$ e sejam $f, l$ e $r$ vértices do $\operatorname{RBC}(c)$ tais que $f$ tem um descendente em $T$ que é vizinho de $w$, e $l$ e $r$ são vértices distintos de $p$ e $f$ tais que $b(l)$ e $b(r)$ são maiores que bp $(w)$ e $l$ e $r$ estão em lados opostos do $\mathrm{RBC}(c)$ determinados por $p$ e $f$ (figura 6.2). 
〈obstrução pelo contador do c-nó 102〉三

void sh_planar_impl::obstruction_edges_cnode_counter(graph\& G, node cnode, node $w$,

$\{$ bool flag $=$ false; node $j l$, node $j r$, node $t 1$, node $t 2)$

node $f=$ nil, $t 12=$ nil, $t 22=$ nil;

if $(t 1 \neq$ nil $\wedge t 1 \neq$ cnode $) t 12=$ last_pnode $(t 1$, cnode $)$;

if $(t 2 \neq$ nil $\wedge t 2 \neq$ cnode $)$ t22 = last_pnode $(t 2$, cnode $)$;

// seachs for a node $\mathrm{f}$ in $\mathrm{RBC}$ [cnode] between $\mathrm{jl}$ and jr s.t.

// $\mathrm{f}$ has a descendant that is a neighbor of $\mathrm{w}$ in $\mathrm{G}$;

myforall $(f, R B C[$ cnode $])$

if $(f \equiv j l \vee f \equiv j r)$ flag $=$ true;

else if $\left(f a g \wedge\left(f \equiv t 12 \vee f \equiv t 22 \vee\right.\right.$ label_b $\left.\left.[f] \equiv d f s p o s_{-} n u m[w]\right)\right)$ break;

if $(f \equiv t 12)$ node_label_b $b]=$ neighbor_w_terminal $[t 1]$;

else if $(f \equiv t 22)$ node_label_b $b]=$ neighbor_w_terminal $[t 2]$;

edge $e=f i n d_{-} e d g e(G$, node_label_b$[f], w)$;

obstruction_edges.append(e);

$e=f i n d \_e d g e\left(G, n o d e \_l a b e l \_b[j l]\right.$, node_with_dfspos $\left.\left[l a b e l \_b[j l]\right]\right)$;

obstruction_edges.append(e);

$e=f i n d \_e d g e\left(G, n o d e_{-} l a b e l \_b[j r], n o d e \_w i t h \_d f s p o s\left[l a b e l \_b[j r]\right]\right) ;$

obstruction_edges.append(e);

node $w 1=$ node_with_dfspos[label_b[jl]], w2 = node_with_dfspos[label_b[jr]];

if (dfspos_num[w2] < dfspos_num[w1]) leda_swap $(w 1, w 2)$;

list_edges_upward_TO(parent[cnode], w2);

list_edges_upward_TO(node_label_b[jl],jl);

list_edges_upward_TO(node_label_b $[j r], j r)$;

list_edges_upward_TO(node_label_b[f], f);

extract_boundary_cycle(cnode, obstruction_edges);

\}

Figura 6.1: Código da função obstruction_edges_cnode_counter. 
Para determinar $f$, a função obstruction_edges_cnode_counter procura, no lado do $\mathrm{RBC}(c)$ determinado por $l$ e $r$ e oposto a $p$, por um vértice cujo valor de $b$ é igual ao $\operatorname{bp}(w)$ ou por um vértice que é ancestral de um terminal. (A função last_pnode determina o último P-nó no caminho de $t$ a $c$ em $T$ e consome tempo proporcional ao comprimento do caminho.)

Sejam $w_{1}$ e $w_{2}$ tais que $\operatorname{bp}\left(w_{i}\right)=b(l)$ ou $\mathrm{bp}\left(w_{i}\right)=b(r)$, para $i=1,2$, e $\mathrm{bp}\left(w_{1}\right) \leq \mathrm{bp}\left(w_{2}\right)$. Sejam $l^{\prime}$ e $r^{\prime}$ os vértices apontados por node_label_b[l] e por node_label_b/r], respectivamente, e seja $u$ um descendente de $f$ que é vizinho de $w$, obtido da seguinte maneira: se $b(f)=\mathrm{bp}(w)$, então $u$ é o vértice apontado por node_label_b $[f]$ (figura 6.2(a)). Caso contrário, $f$ é um ancestral de um terminal $t$ (figura 6.2(b)), e $u$ é o vértice apontado por neighbor_w_terminal[t].

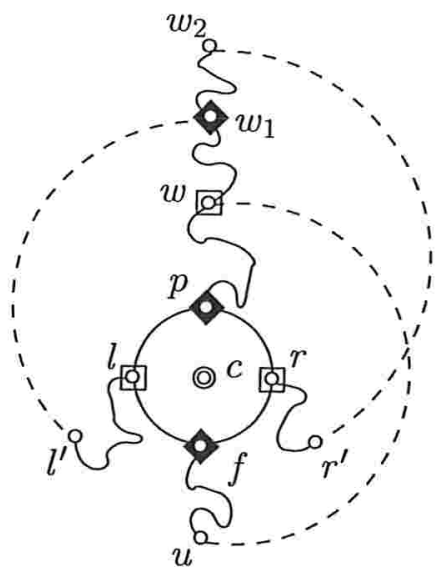

(b)

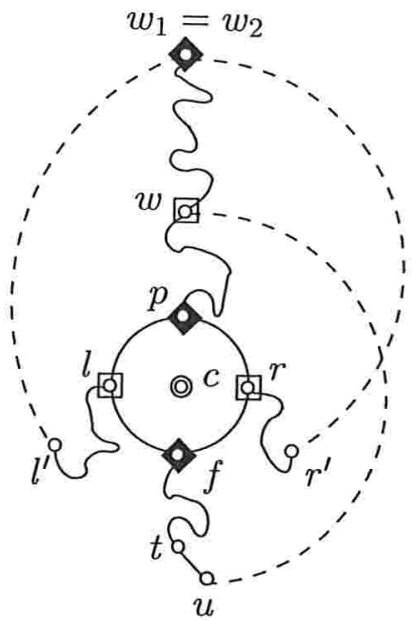

(a)

Figura 6.2: Obstrução detectada pela função test_cnode_counter, onde $p$ é o pai do C-nó $c, w_{1}$ e $w_{2}$ são ancestrais de $w$ em $T$ e $l$ e $r$ são vértices em $\operatorname{RBC}(c)$ cujos valores de $b$ são maiores que $\operatorname{bp}(w)$. (a) $f$ é um vértice em $\operatorname{RBC}(c)$ cujo valor de $b$ é igual ao $\mathrm{bp}(w)$. (b) $f$ é um vértice em $\mathrm{RBC}(c)$ que é ancestral de um terminal $t$.

A lista dos arcos de uma obstrução é representada pela variável global obstruction_edges. Neste caso, ela é construída da seguinte maneira.

Primeiramente, os arcos $u w, l^{\prime} w_{i}$ e $r^{\prime} w_{j}(i$ e $j$ em $\{1,2\})$ são adicionados a essa lista. Para encontrar um arco $x y$ em $G$, usa-se a função find_edge (figura 6.3), que percorre a lista de adjacência de $x$ e devolve um apontador para o arco $x y$, caso exista, ou nil caso contrário.

Feito isso, adiciona-se a essa lista, os arcos dos seguintes caminhos em $T_{0}$ : de $p$ a $w_{2}$, de $l^{\prime}$ a $l$, de $r^{\prime}$ a $r$ e de $u$ a $f$. A adição dos arcos de um caminho em $T_{0}$ de $x$ a $y$ à lista obstruction_edges é feita pela rotina list_edges_upward_TO (figura 6.4). Para cada vértice $v$ distinto de $y$ nesse caminho, essa função adiciona o arco $v^{\prime} v$ a obstruction_edges, onde $v^{\prime}$ é o pai de $v$ em $T_{0}$. 
104a 〈procura arco 104a) $\equiv$

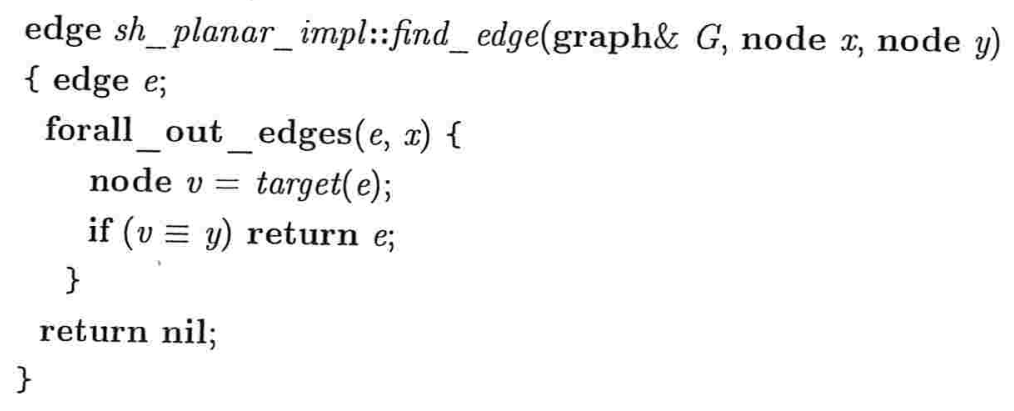

Figura 6.3: Código da função find_edge.

104b 〈adiciona arcos subindo em T0 104b) 三

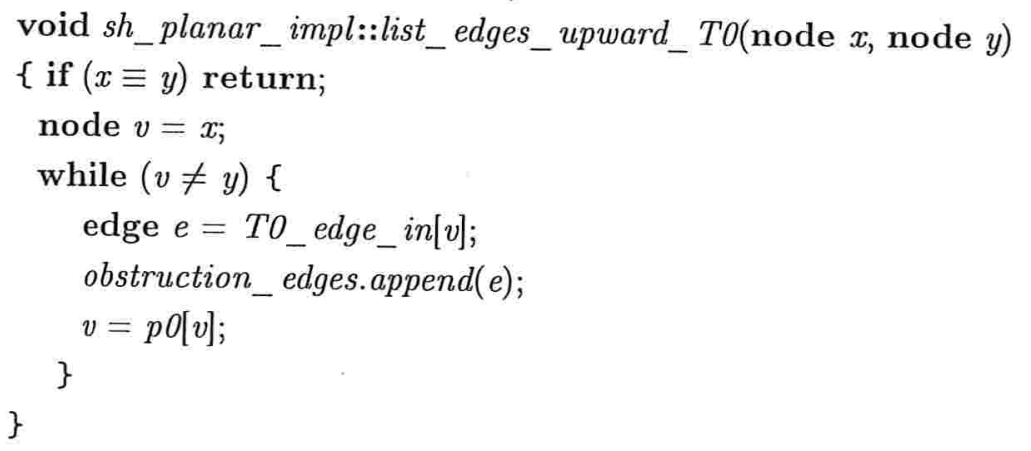

Figura 6.4: Código da função list_edges_upward_TO. 
Finalmente, os arcos do ciclo facial externo de $c$ são adicionados à lista obstruction_edges pela rotina extract_boundary_cycle (figura 6.5), que primeiramente calcula as listas de adjacências do bloco em $H+w$ representado por $c$ através da lista $\operatorname{DCP}(c)$, representada pela variável global embed_list $[c]$, e depois obtém o ciclo facial determinado por $\mathrm{DCP}(c)$ e $p$.

〈lista de arcos na fronteira da face externa 105〉三

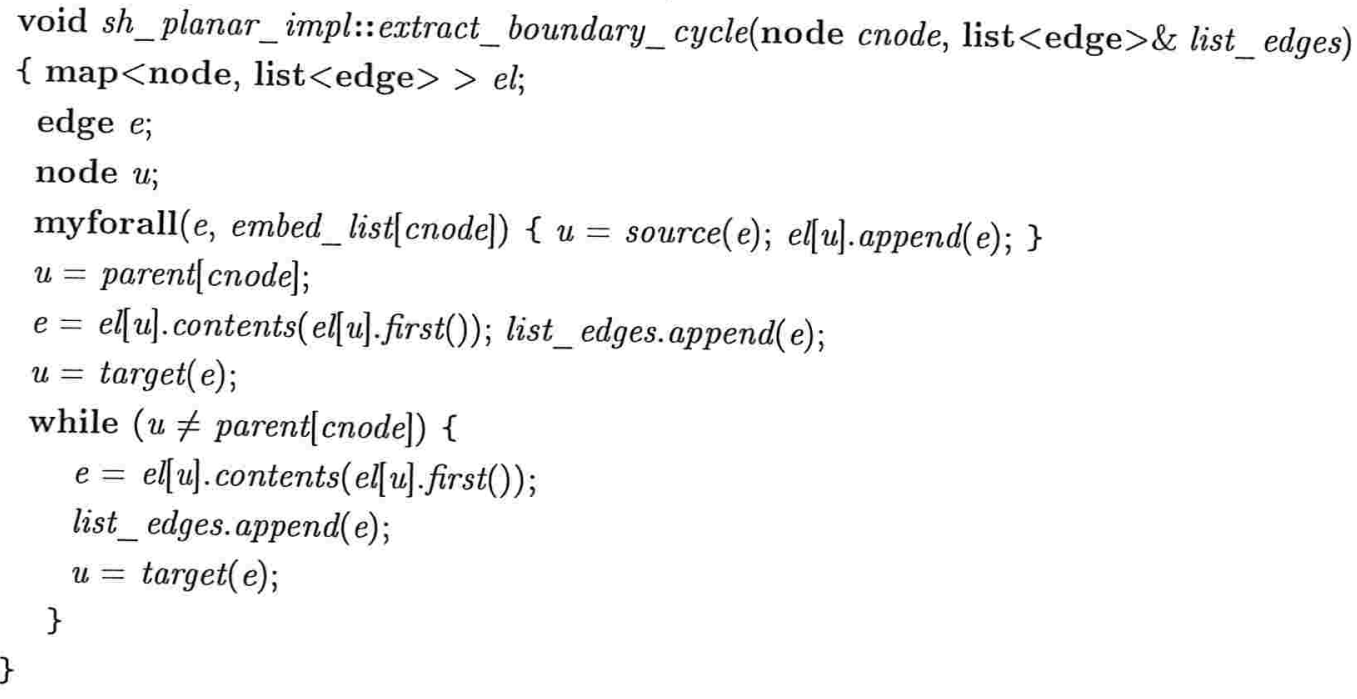

Figura 6.5: Código da função extract_boundary_cycle.

\subsection{Obstrução para cnode_of_possible_k33_obstruction}

Seja $d$ o C-nó (ativo) apontado pela variável global cnode_of_possible_k33_obstruction. Conforme descrito na seção $4.7, d$ é o primeiro C-nó detectado nas condições da figura 4.14(b) (página 66) pela função test_cnode_counter.

Seja $Q$ o caminho de um terminal $t$ a $w$ em $T$ e seja $Q^{\prime}$ o caminho do pai de $d$ a $w$ em $T$. Existe uma obstrução do tipo (o1) se, para um nó $u$ em $Q^{\prime}, u$ é vizinho de um vértice em $L \backslash\{w\}$ ou $u$ tem um filho (P-nó) $v$ em $T$ que não está em $Q$ e $v$ tem um descendente em $T$ que é vizinho de um vértice em $L \backslash\{w\}$ (figura 4.14(c)). (Observe que, no caso de dois terminais, $v$ pode ser simplesmente o terminal $t^{\prime}$ distinto de $t$, se $t^{\prime}$ é um P-nó, ou o pai de $t^{\prime}$ em $T$, caso contrário.)

A função obstruction_edges_possible_obstr_confirmed (figura 6.6) é acionada pela função find_obstruction (pontos marcados com (2) nas figuras 4.11 e 4.12, páginas 63 e 64) para calcular uma lista de arcos de uma obstrução do tipo (o1), caso exista.

Sejam $p$ o pai de $d$ e $l, r$ e $f$ como mostrados na figura 6.7. Sejam $w_{1}$ e $w_{2}$ tais que $\mathrm{bp}\left(w_{i}\right)=b(p)$ ou $\mathrm{bp}\left(w_{i}\right)=b(f)$, para $i=1,2$, e $\mathrm{bp}\left(w_{1}\right) \leq \mathrm{bp}\left(w_{2}\right)$. Seja $l^{\prime}$ o vértice apon- 
〈obstrução pelo contador do c-nó 102〉+三

void sh_planar_impl::obstruction_edges_possible_obstr_confirmed(graph\& G,

$\{$ node cnode $=$ cnode_of_possible_k33_obstruction, node $w$, node $t$, node $v$ )

$f=$ obstruction_nodes.pop ()$, j l=$ obstruction_nodes.pop ()$, j r=$ obstruction_nodes.pop () ;

if $($ label_b $\left.[j]]>d f s p o s_{-} n u m[w]\right)$ node_label_b $[j l]=$ neighbor_w_terminal $[t]$;

if $\left(l a b e l_{-} b[j r]>d f s p o s_{-} n u m[w]\right)$ node_label_b $[j r]=n e i g h b o r_{-} w_{-}$terminal $[t] ;$

edge $e=f i n d \_e d g e(G$, node_label_b $\left.b j], w\right)$;

obstruction_edges.append(e);

$e=f i n d_{-} e d g e\left(G, n o d e_{-} l a b e l_{-} b[j r], w\right)$;

obstruction_edges.append(e);

$e=f i n d \_e d g e\left(G, n_{0} d_{-} l a b e l_{-} b[f]\right.$, node_with_dfspos $[$ label_b $[f])$;

obstruction_edges.append(e);

$e=f i n d_{-} e d g e\left(G, n o d e_{-} l a b e l_{-} b[v], n o d e_{-} w i t h \_d f s p o s\left[l a b e l \_b[v]\right]\right) ;$

obstruction_edges.append(e);

node $w 1=$ node_with_dfspos[label_b[v]], w2 = node_with_dfspos[label_b[f]];

if $\left(d f s p o s \_n u m[w 2]<d f s p o s \_n u m[w 1]\right)$ leda_swap $(w \overline{1}, w 2)$;

list_edges_upward_TO $(w, w 2)$;

list_edges_upward_TO(node_label_b[jl],jl);

list_edges_upward_TO(node_label_b $[j r], j r)$;

list_edges_upward_TO(node_label_b[f], $f)$;

node $m=l c a \_b e t w e e n($ parent $[$ cnode $], v, p 0)$;

list_edges_upward_TO(parent [cnode], m);

list_edges_upward_TO(node_label_b $[v], m)$;

\}

extract_boundary_cycle(cnode, obstruction_edges);

Figura 6.6: Código da função obstruction_edges_possible_obstr_confirmed. 
tado por node_label_b[l], se $b(l)=\mathrm{bp}(w)$, ou o vértice apontado por neighbor_w_terminal $[t]$, onde $t$ é um terminal que é descendente de $l$, caso contrário. Analogamente, seja $r^{\prime}$ o vértice apontado por node_label_b $r]$, se $b(r)=\mathrm{bp}(w)$, ou o vértice apontado por neighbor_w_terminal $\left[t^{\prime}\right]$, onde $t^{\prime}$ é um terminal que é descendente de $r$, caso contrário. Seja $u$ o vértice apontado por node_label_b[f].

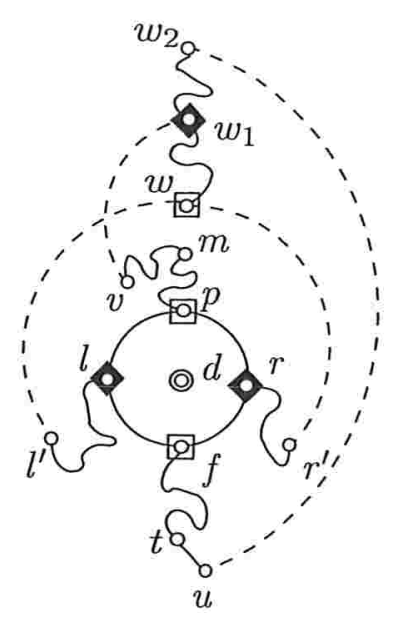

Figura 6.7: Obstrução detectada pelo teste de $Q^{\prime}$ da figura 4.14(c) (página 66), para o caso de um terminal, ou pela presença de um C-nó $d$ como na figura 4.14(b), para o caso de dois terminais.

Seja $v$ o P-nó devolvido pela função find_node_with_label_b_greater_than_dfs_w (figura 4.17, página 69) para o caso de um terminal. Para o caso de dois terminais, seja $t^{\prime}$ o terminal distinto de $t$. Neste caso, $v=t^{\prime}$, se $t^{\prime}$ é um P-nó (figura 6.8(a)). Caso $t^{\prime}$ seja um C-nó, $v$ é o pai (P-nó) de $t^{\prime}$ e copia-se as informações das variáveis node_label_b[ ] e label_b[] de $t^{\prime}$ para $v$, pois $t^{\prime}$ pode ser um C-nó terminal tal que $b\left(t^{\prime}\right)<b(t)$ e $v$ é o ancestral mais baixo entre os dois terminais em $T$ (figura 6.8(b)). Finalmente, seja $m$ o ancestral mais baixo entre $p$ e $v$ em $T_{0}$.

A lista dos arcos para esta obstrução é construída da seguinte maneira. Primeiramente, os $\operatorname{arcos} l^{\prime} w, r^{\prime} w, u w_{i}$ e $v w_{j}$, onde $\operatorname{bp}\left(w_{i}\right)=b(u)$ e $\operatorname{bp}\left(w_{j}\right)=b(v)$ ( $i$ e $j$ em $\left.\{1,2\}\right)$, são adicionados a essa lista. Em seguida, adiciona-se a esta lista, os arcos dos seguintes caminhos em $T_{0}$ : de $w$ a $w_{2}$, de $l^{\prime}$ a $l$, de $r^{\prime}$ a $r$, de $u$ a $f$, de $p$ a $m$ e de $v$ a $m$. Por fim, os arcos do ciclo facial externo de $c$ são adicionados à lista obstruction_edges.

\subsection{Cálculo de uma subdivisão de $K_{5}$}

A função obstruction_edges_K5 (figura 6.9) é acionada pela função find_obstruction nos pontos marcados com (3) e (4) nas figuras 4.11 e 4.12 (páginas 63 e 64) para o caso de calcular uma subdivisão de $K_{5}$ em $G$. Seja $c$ o C-nó para o qual foi detectada uma obstrução $\left(C, v_{1}, v_{2}, v_{3}\right)$ do 


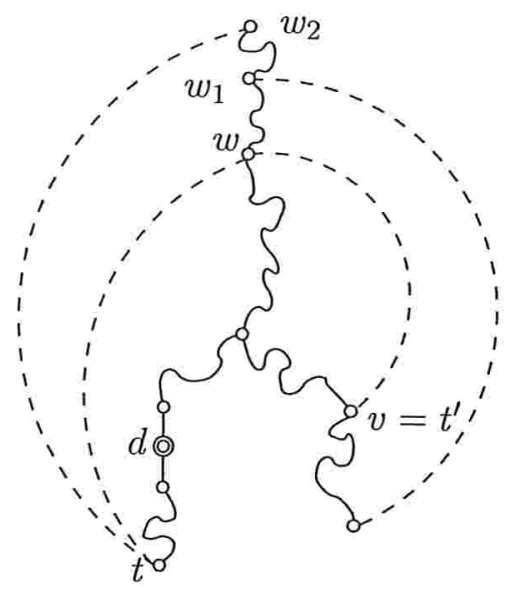

(a)

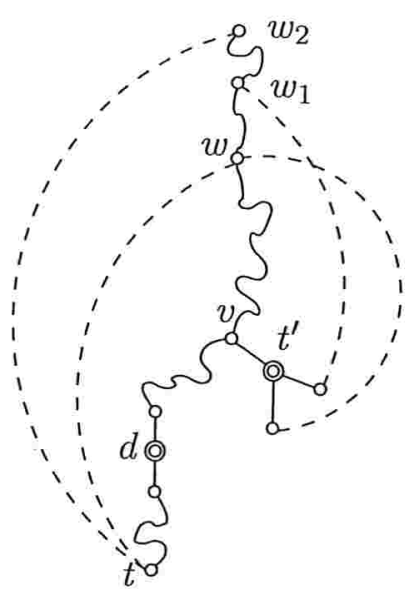

(b)

Figura 6.8: O C-nó $d$ é o C-nó apontado por cnode_of_possible_k33_obstruction, e $t$ e $t^{\prime}$ são dois terminais. (a) $v$ coincide com o P-nó terminal $t^{\prime}$. (b) $v$ é o pai do C-nó terminal $t^{\prime}$.

tipo (o2) e seja $w_{1}$ o vértice de menor bp-número entre os vértices apontados por node_label_b $\left.v_{i}\right]$, para $i=1,2,3$. A função obstruction_edges_K5 (figura 6.9) é acionada se

$\left(K_{5} 1\right)$ ou $v_{i}$ é um terminal ou $v_{i}=p$, onde $p$ é o pai de $c$ e existe um descendente $v$ de $p$ em $T$ que não é descendente de $c$ e é vizinho de um vértice em $L \backslash\{w\}$;

$\left(K_{5} 2\right)$ pelo menos dois vértices de $\left\{v_{1}, v_{2}, v_{3}\right\}$ têm o apontador node_label_b [ ] para $w_{1}$ (figuras $6.10(a)$ e (b)).

Observe que, se algum $v_{i}$ é o pai de um C-nó terminal, também é possível construir uma subdivisão de $K_{5}$ em $G$ (figura $6.10(\mathrm{c})$ ). Mas neste caso, podemos obter uma subdivisão de $K_{3,3}$, conforme é descrito mais adiante.

Seja $w_{2}$ o vértice de maior bp-número entre os vértices apontados por node_label_b $\left[v_{i}\right]$, para $i=1,2,3$. A lista dos arcos para esta obstrução é construída da seguinte maneira. Primeiramente, adiciona-se a esta lista, os arcos do caminho de $w$ a $w_{2}$ em $T_{0}$. Em seguida, os arcos do ciclo facial externo de $c$ são adicionados à lista obstruction_edges.

Finalmente, se $v_{i}=p$ para algum $i$ em $\{1,2,3\}$, então seja $v$ o vértice apontado por node_label_b $\left[v_{i}\right]$. A função obstr_edges_pnode (figura 6.11) adiciona à lista obstruction_edges o arco $v w_{j}(j$ em $\{1,2\})$ e os arcos no caminho de $v$ a $w$ em $T_{0}$.

Se $v_{i}$ é um P-nó terminal, então sejam $u_{1}$ e $u_{2}$ os vértices apontados por neighbor_w_terminal $\left[v_{i}\right]$ e por node_label_b $\left[v_{i}\right]$, respectivamente. A função obstr_edges_terminal (figura 6.12) adiciona os arcos $u_{1} w$ e $u_{2} w_{j}$ à lista obstruction_edges, além de adicionar os caminhos de $u_{1}$ a $v_{i}$ e de $u_{2}$ a $v_{i}$ em $T_{0}$ nessa lista. 
$109 \mathrm{a}$

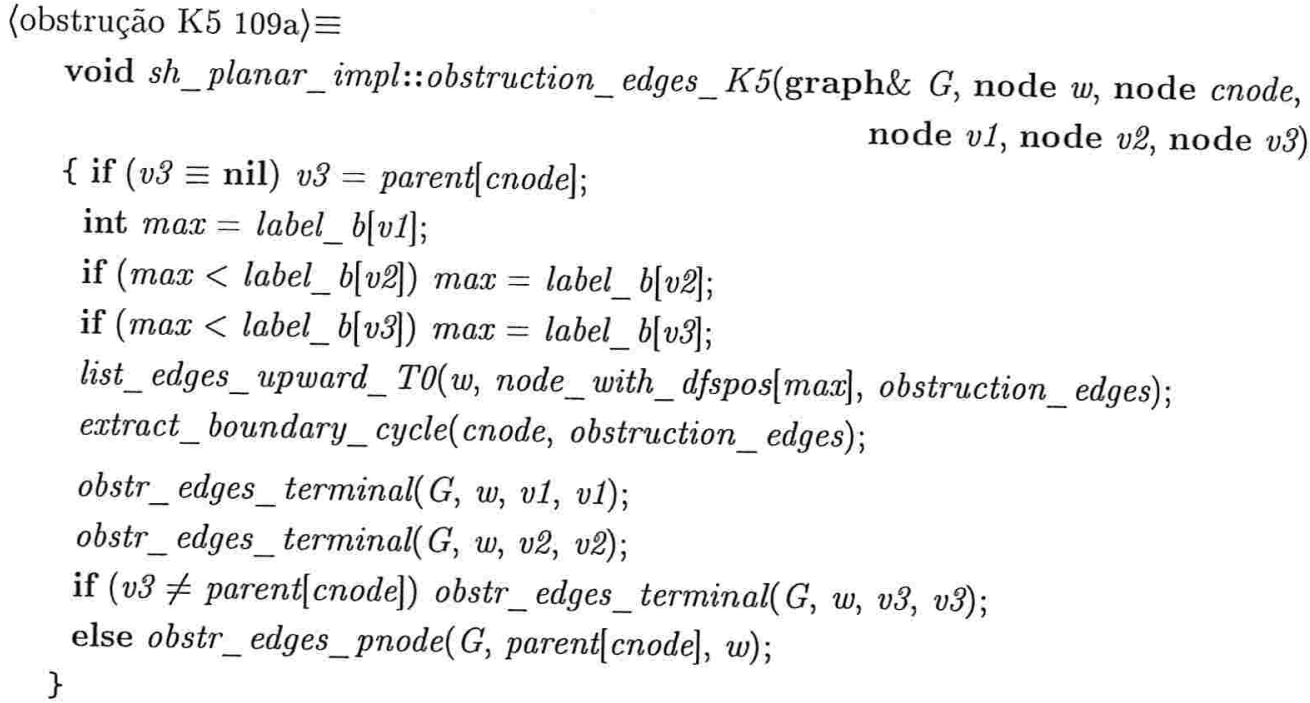

Figura 6.9: Código da função obstruction_edges_K5.

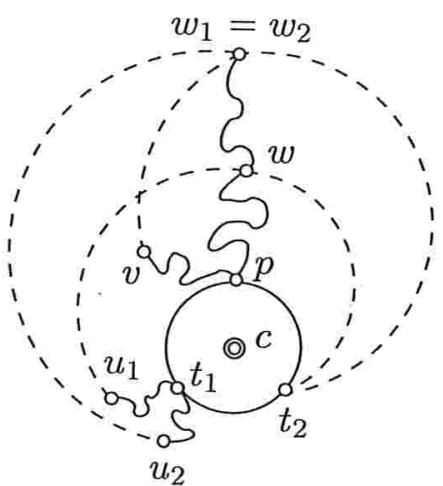

(a)

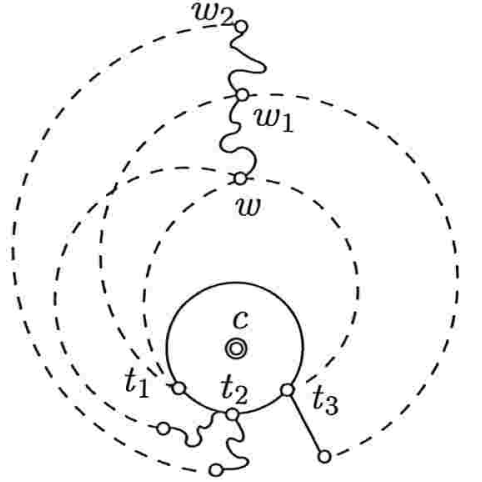

(b)

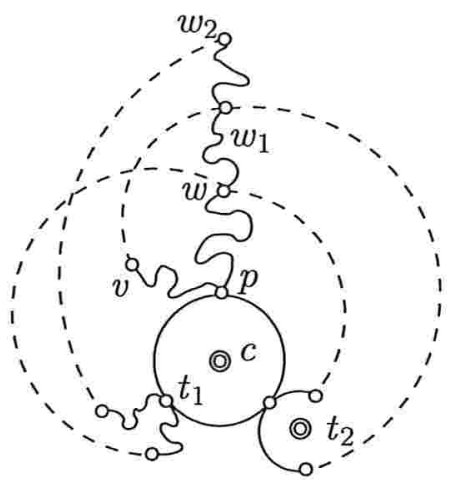

(c)

Figura 6.10: Cálculo de uma subdivisão de $K_{5}$. (a) Caso de dois terminais $t_{1}$ e $t_{2}$. (b) Caso em que ocorre três terminais $t_{1}, t_{2}$ e $t_{3}$. (c) $t_{2}$ é um C-nó terminal.

109b 〈arcos do pai de um c-nó 109b〉三

void sh_planar_impl::obstr_edges_pnode(graph\& $G$, node $p$, node $u)$

$\left\{\right.$ edge $e=f i n d \_e d g e\left(G, n o d e \_l a b e l \_b[p]\right.$, node_with_dfspos $[$ label_b $\left.[p]]\right)$; obstruction_edges.append(e);

Figura 6.11: Código da função obstr_edges_pnode. 
$\langle\operatorname{arcos}$ em T0 do terminal e arcos de retorno 110$\rangle \equiv$

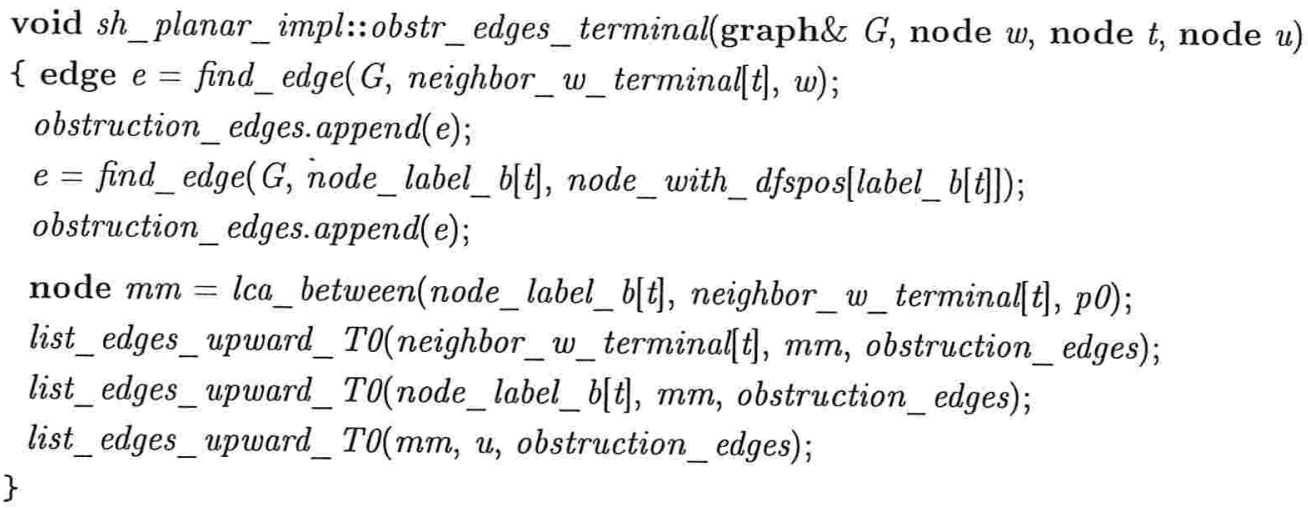

Figura 6.12: Código da função obstr_edges_terminal.

Na seção 6.5 a seguir, é descrita a construção de uma subdivisão de $K_{3,3}$ no caso de apenas a condição $\left(K_{5} 1\right)$ ser satisfeita. Já nas seções seguintes (6.6 e 6.7), uma subdivisão de $K_{3,3}$ é construída no caso da condição $\left(K_{5} 1\right)$ não ser satisfeita.

\subsection{Subdivisão de $K_{3,3}$ devido ao grau de $w 1$}

A função obstruction_edges_countmin_1 (figura 6.13) é acionada pela função find_obstruction nos pontos marcados com (3) e (4) nas figuras 4.11 e 4.12 (páginas 63 e 64) para calcular uma subdivisão de $K_{3,3}$ em $G$ para uma obstrução $\left(C, v_{1}, v_{2}, v_{3}\right)$ do tipo (o2). Seja $c$ o C-nó para o qual foi detectada a obstrução e seja $w_{1}$ o vértice de menor bp-número entre os vértices apontados por node_label_b $\left[v_{i}\right]$, para $i=1,2,3$. Neste caso daria para construir uma subdivisão de $K_{5}$ em $G$ se não fosse pela presença de um único vértice em $\left\{v_{1}, v_{2}, v_{3}\right\}$ tal que variável node_label_b $\left[v_{i}\right]$ aponta para $w_{1}$ (figura 6.14). A seguir encontra-se uma descrição da função obstruction_edges_countmin_1.

Primeiramente, os vértices $v_{i}$ são ordenados em ordem não decrescente de $b$ pela função sort_by_label_b (figura 6.15). Nela, a função leda_swap $(x, y)$ é usada para trocar os valores de $x$ com o de $y$. Para $i=1,2,3$, seja $w_{i}$ o vértice de bp-número igual a $b\left(v_{i}\right)$. São adicionados à lista obstruction_edges os arcos do caminho de $w$ a $w_{3}$ em $T_{0}$, pela função list_edges_upward_TO, e os arcos no lado do ciclo facial externo de $c$, determinado por $v_{2}$ e $v_{3}$, que contém $v_{1}$, pela função add_part_of_bc (figura 6.16).

Em seguida, seja $v$ o vértice apontado por node_label_b $\left[v_{1}\right]$. Adiciona-se à obstruction_edges o arco $v w_{1}$ e os arcos no caminho de $v$ a $v_{1}$ em $T_{0}$.

Finalmente, para $i=2,3, v_{i}$ é ou o pai de $c$ ou é um P-nó terminal e adiciona-se os arcos 
〈obstrução de três no mesmo c-nó 111) 三

void sh_planar_impl::obstruction_edges_countmin_1(graph\& $G$, node $w$, node cnode,

$\{$ if $(v 3 \equiv$ nil $) v 3=$ parent $[$ cnode $]$; node $v 1$, node $v 2$, node $v 3$ )

sort_by_label_b(v1,v2, v3);

list_edges_upward_TO $(w$, node_with_dfspos[label_b[v3]]);

add_part_of_bc(cnode, v1, v2, v3);

edge $e=f i n d \_e d g e(G$, node_label_b[v1], node_with_dfspos $[$ label_b[v1]]);

obstruction_edges.append(e);

list_edges_upward_TO(node_label_b[v1],v1);

if $(v 2 \neq$ parent $[$ cnode $])$ obstr_edges_terminal $(G, w, v 2, v 2)$;

else obstr_edges_pnode( $G$, parent [cnode], $w)$;

if $(w 3 \neq$ parent $[$ cnode $])$ obstr_edges_terminal $(G, w, v 3, v 3)$;

else obstr_edges_pnode( $G$, parent[cnode], $w)$;

\}

Figura 6.13: Código da função obstruction_edges_countmin_1.

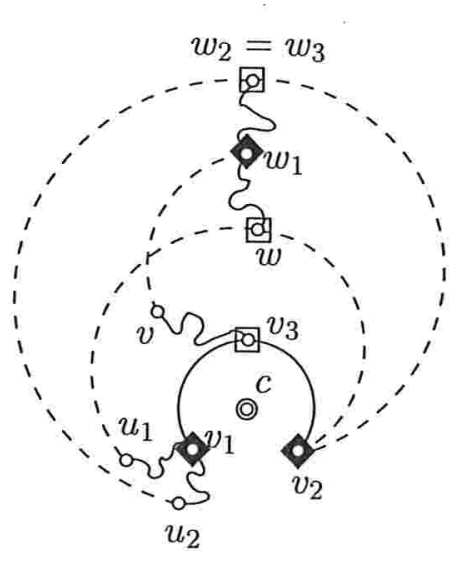

(a)

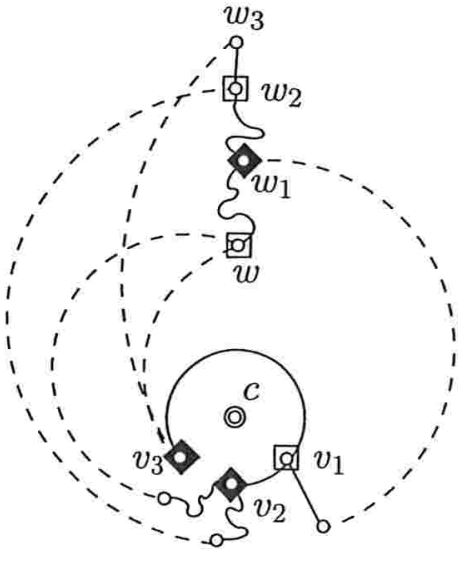

(b)

Figura 6.14: Cálculo de uma subdivisão de $K_{3,3}$. Neste caso, não é possível obter uma subdivisão de $K_{5}$ pois $w_{1}$ não tem grau 4. (a) Caso de dois terminais $v_{1}$ e $v_{2}\left(v_{3}\right.$ é o pai de $\left.c \operatorname{em} T\right)$. (b) Caso de três terminais $v_{1}, v_{2}$ e $v_{3}$. 
112a 〈ordena nós pelo valor de b 112a) 三

void $s h \_p l a n a r+i m p l:: s o r t \_b y \_l a b e l \_b($ node $\& t 1$, node $\& t 2$, node \& $t 3)$

$\{$ if $($ label_b $[t 2]<$ label_b $[t 1])$ leda_swap $(t 1, t 2)$;

if $($ label_b $[t 3]<$ label_b $b 1])$ leda_swap $(t 1, t 3)$;

if $\left(l a b e l \_b[t 3]<l_{0} a b e l_{-} b[t 2]\right)$ leda_swap $(t 2, t 3)$;

\}

Figura 6.15: Código da função sort_by_label_b.

112b 〈alguns arcos na fronteira da face externa 112b) 三

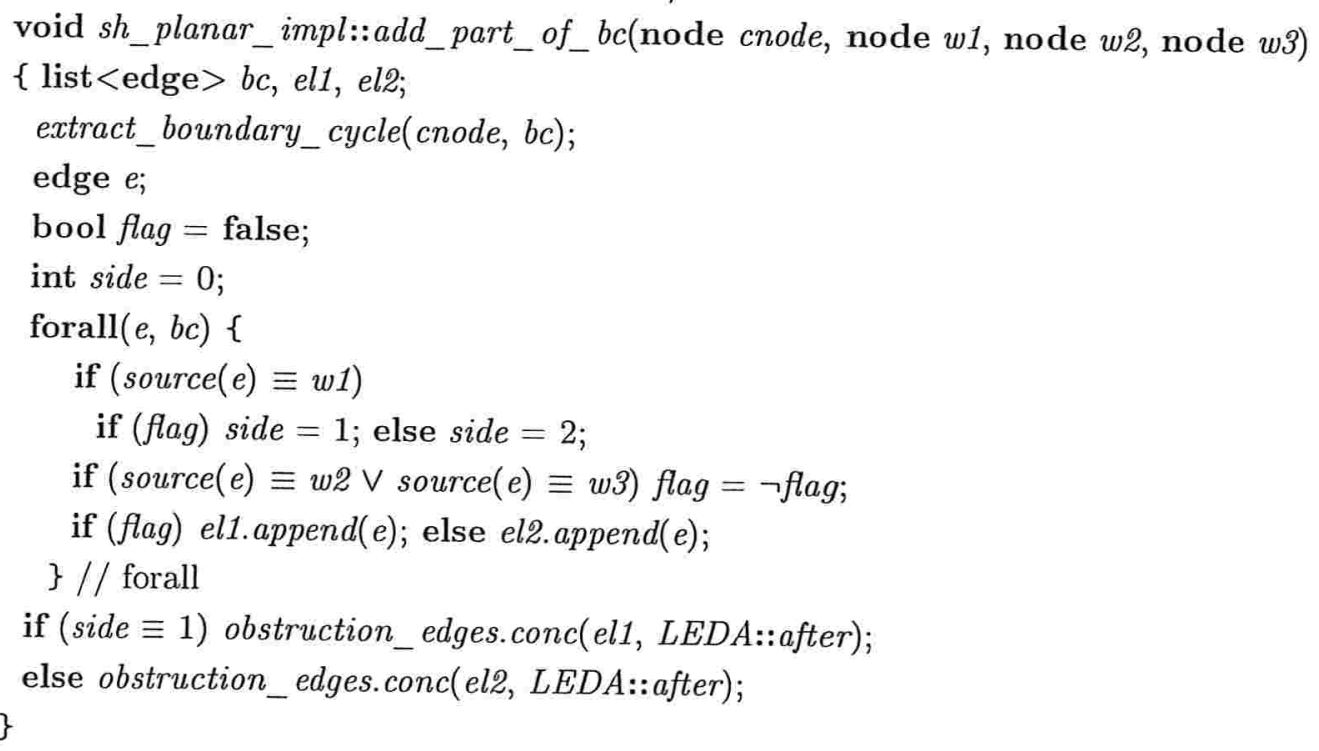

Figura 6.16: Código da função add_part_of_bc. 
devidos a $v_{i}$ da mesma maneira que foi descrita na seção anterior, através das funções obstr_edges_pnode e obstr_edges_terminal, respectivamente.

\subsection{Subdivisão de $K_{3,3}$ com parte do ciclo externo de um C-nó}

Seja $\left(C, v_{1}, v_{2}, v_{3}\right)$ uma obstrução do tipo (o2) detectada para um C-nó $c$ e seja $m$ o ancestral comum em $T_{0}$ de pelo menos dois entre $v_{1}, v_{2}$ e $v_{3}$, com o menor bp-número. A função obstruction_edges_countmin_2_3 (figura 6.17) é acionada pela função find_obstruction nos pontos marcados com (3) e (4) nas figuras 4.11 e 4.12 (páginas 63 e 64) para calcular uma subdivisão de $K_{3,3}$ em $G$ se a condição ( $\left.K_{5} 1\right)$ não é satisfeita e se $m$ coincide com um terminal (figura 6.18).

〈obstrução de dois no mesmo c-nó 113) 三

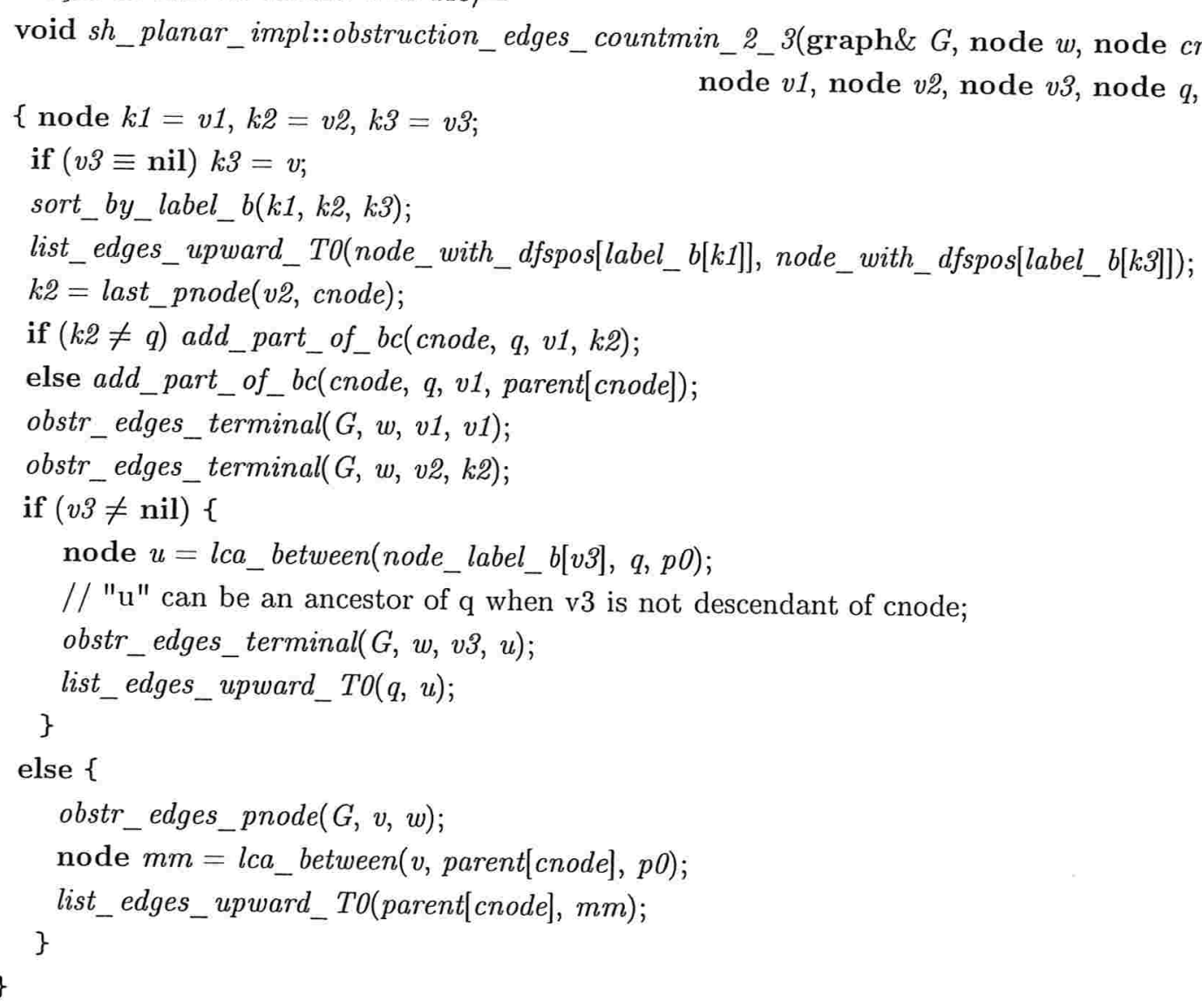

Figura 6.17: Código da função obstruction_edges_countmin_2_3.

Seja $q$ um vértice tal que, se $v_{i}=p$ para algum $i$ em $\{1,2,3\}$, então $q$ coincide com $p$ (figura 6.18(a)). Caso contrário, $q$ coincide com $v_{j}, j$ em $\{1,2,3\}$, tal que $v_{j}$ não é terminal (figura 6.18(b)). A lista de arcos da obstrução é calculada da seguinte maneira. 


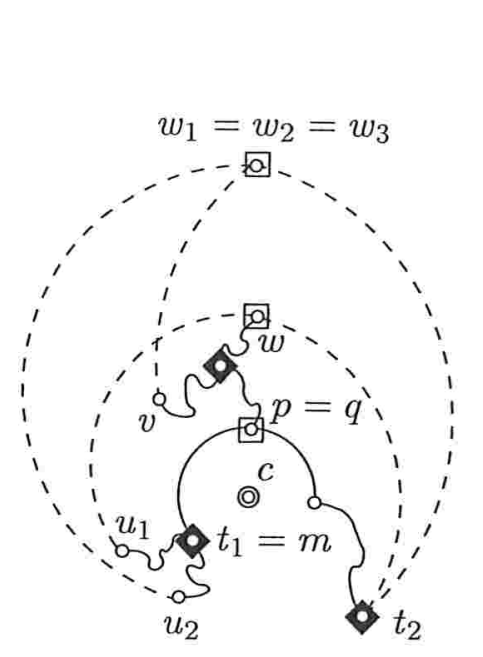

(a)

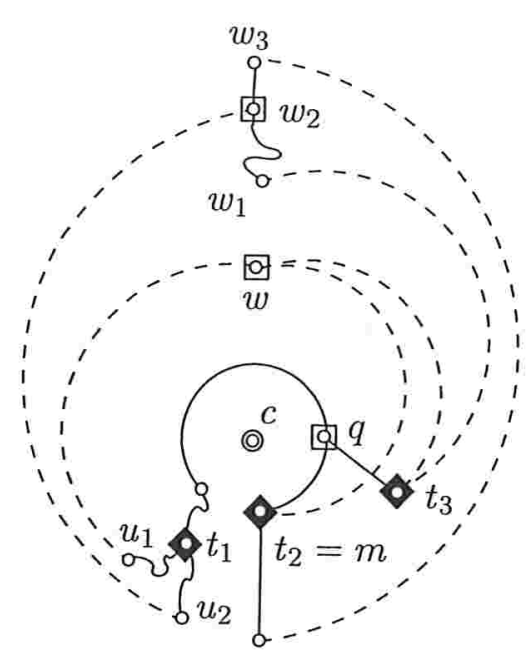

(b)

Figura 6.18: Cálculo de uma subdivisão de $K_{3,3}$ com um "pedaço" do ciclo facial externo de um C-nó c. (a) Caso de dois terminais $t_{1}$ e $t_{2}$. (b) Caso de três terminais $t_{1}, t_{2}$ e $t_{3}$.

Primeiramente, os vértices $v_{1}, v_{2}$ e $v_{3}$ são ordenados em ordem não-decrescente de $b$ e, para $i=1,2,3$, seja $w_{i}$ o vértice de bp-número igual a $b\left(v_{i}\right)$. Adiciona-se à lista obstruction_edges os arcos do caminho de $w_{1}$ a $w_{3}$ em $T_{0}$ e os arcos no lado do ciclo facial externo de $c$ que contém $q$, determinado por $v_{j}$ e $v_{k}$, onde $j$ e $k$ estão em $\{1,2,3\}$ e $j \neq k, v_{j} \neq q$ e $v_{k} \neq q$. (A função last_pnode (x,y) devolve o último P-nó no caminho de $x$ a $y$ em $T$, caso exista.)

Para finalizar, adiciona-se os arcos devidos a $v_{i}$ da seguinte maneira. Se $v_{i}$ coincide com $p$, então adiciona-se os arcos devidos a $v_{i}$ conforme descrito na seção 6.4, através da função obstr_edges_pnode. Caso contrário, seja $t_{i}$ o terminal que é descendente de $v_{i}$ em $T$ e sejam $u_{1}$ e $u_{2}$ os vértices apontados por neighbor_w_terminal $\left.t_{i}\right]$ e por node_label_b $\left.t_{i}\right]$, respectivamente. A função obstr_edges_terminal adiciona os $\operatorname{arcos} u_{1} w$ e $u_{2} w_{i}$ à lista obstruction_edges, além de adicionar os caminhos em $T_{0}$ de $u_{1}$ a $t_{i}$, de $u_{2}$ a $t_{i}$ e de $t_{i}$ a $v_{i}$ nessa lista.

\subsection{Subdivisão de $K_{3,3}$, subindo em $T_{0}$}

A função obstruction_edges_T0 (figura 6.19) é acionada pela função find_obstruction nos pontos marcados com (3) e (4) nas figuras 4.11 e 4.12 (páginas 63 e 64) para calcular uma subdivisão de $K_{3,3}$ em $G$ ou devido a uma obstrução do tipo (o3) ( $m, K_{1}, K_{2}, K_{3}$ ) (figuras 6.20(a) e (b)), ou devido a uma obstrução do tipo (o2) $\left(C, v_{1}, v_{2}, v_{3}\right)$ tal que a condição $\left(K_{5} 1\right)$ não é satisfeita e o ancestral comum $m$ em $T_{0}$ de pelo menos dois entre $v_{1}$, $v_{2}$ e $v_{3}$ com o menor bp-número não é um terminal (figura $6.20(\mathrm{c})$ ). 
〈obstrução por T0 115〉三

void sh_planar_impl::obstruction_edges_T0(graph\& G, node $w$, node parent_cnode,

$\{$ if $(t 3 \equiv$ nil $) t 3=v$; node $t 1$, node $t 2$, node $t 3$, node $v$ )

node $q 1=t 1, q 2=t 2, q 3=t 3$

sort_by_label_b( $q 1, q 2, q 3)$;

list_edges_upward_TO(node_with_dfspos[label_b[q1]], node_with_dfspos[label_b[q3]]);

$q 1=t 1 ; q 2=t 2 ; q 3=t 3 ;$

if $\left(i s_{-} \operatorname{cnode}(q 1)\right) q 1=\operatorname{parent}[q 1]$;

if (is_cnode (q2)) q2 = parent [q2];

if $($ is_cnode( $(q 3))$ q3 $=$ parent $[q 3]$;

node $m 1=l c a \_b e t w e e n(q 1, q 2, p 0), m 2=l c a \_b e t w e e n(q 1, q 3, p 0), m 3=l c a \_b e t w e e n(q 2, q 3, p 0)$;

int $\min =d f s p o s_{-} n u m[m 1], \max =d f s p o s_{-} n u m[m 1]$

if $($ dfspos_num $[\mathrm{m} 2]<\min )\left\{\right.$ leda_swap $\left.(t 2, t 3) ; \min =d f s p o s \_n u m[m 2] ;\right\}$

if (dfspos_num [m3] < min) \{

node $k 1=t 2, k 2=t 3, k 3=t 1$;

$t 1=k 1 ; t 2=k 2 ; t 3=k 3$;

\}

$\min =d f s p o s_{-} n u m[\mathrm{~m} 3]$;

if (dfspos_num[m2] $>$ max) $\max =$ dfspos_num[m2];

if (dfspos_num[m3] > max) max $=$ dfspos_num[m3];

obstr_edges_terminal $(G, w, t 1$, node_with_dfspos $[$ max $])$;

obstr_edges_terminal $(G, w, t 2$, node_with_dfspos $[\mathrm{min}])$;

if $(t 3 \neq v)$ obstr_edges_terminal $(G, w, t 3$, node_with_dfspos $[$ max $])$;

else \{

node $u=w$;

if $\left(l c a \_b e t w e e n\left(v, n o d e \_w i t h \_d f s p o s[m i n], p 0\right) \equiv\right.$ node_with_dfspos $[$ min $\left.]\right)$

$\{/ / \mathrm{v}$ is in the same c-node of node_with_dfspos[min];

node cnode $=$ active_cnode_of $($ true, $v)$;

add_part_of_bc(cnode, parent[cnode], v, node_with_dfspos[min] $)$;

$u=v$;

list_edges_upward_TO(parent $[$ cnode $], w)$;

\}

obstr_edges_pnode $(G, v, u)$;

\}

\}

Figura 6.19: Código da função obstruction_edges_TO. 


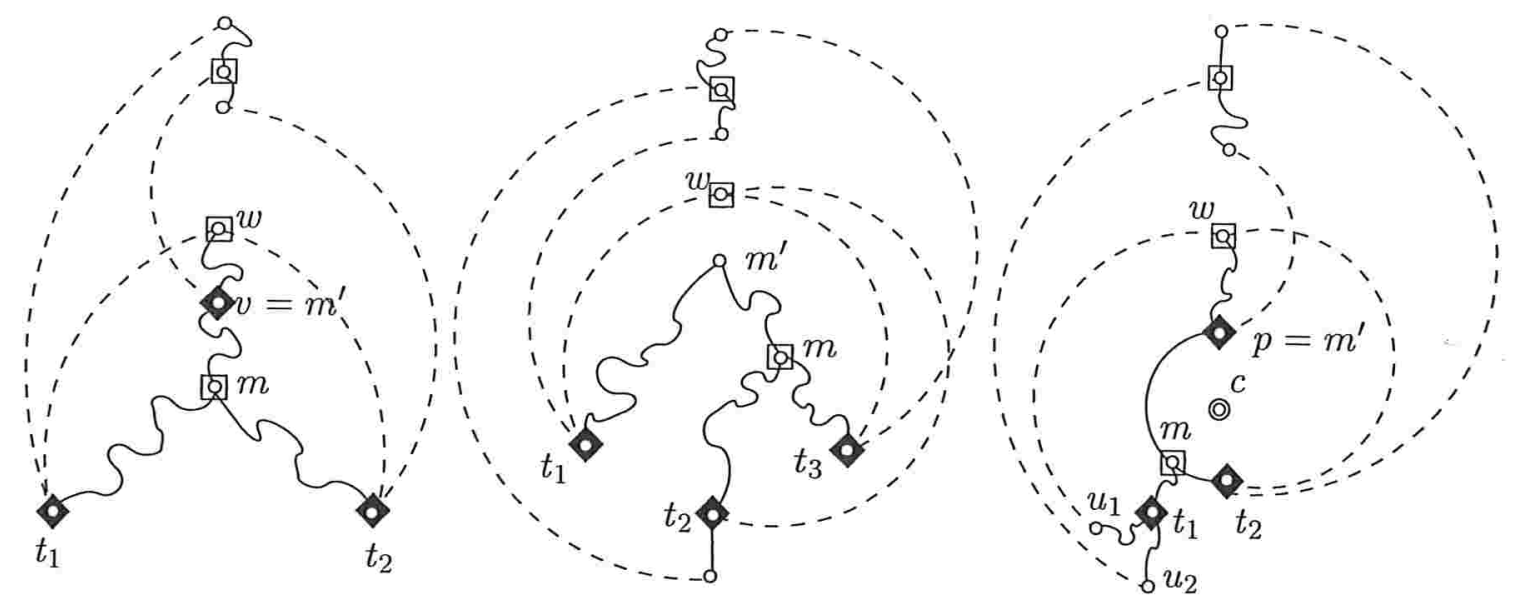

(a)

(b)

(c)

Figura 6.20: Cálculo de uma subdivisão de $K_{3,3}$ subindo em $T_{0}$. (a) Caso de três terminais $t_{1}, t_{2}$ e $t_{3}$. (b) Caso de dois terminais $t_{1}$ e $t_{2}$.

Sejam $q_{1}, q_{2}$ e $q_{3}$ vértices distintos tais que, para $i=1,2,3, q_{i}$ coincide com um terminal ou com o vértice $v$ devolvido pela função find_node_with_label_b_greater_than_dfs_w. A lista de arcos da obstrução é construída da seguinte maneira. Primeiramente, os vértices $q_{1}, q_{2}$ e $q_{3}$ são ordenados em ordem não-decrescente de $b$ e, para $i=1,2,3$, seja $w_{i}$ o vértice de bp-número igual a $b\left(q_{i}\right)$. Os arcos no caminho de $w_{1}$ a $w_{3}$ em $T_{0}$ são adicionados à lista obstruction_edges.

Sejam $m$ e $m^{\prime}$ os ancestrais comuns em $T_{0}$ de pelo menos dois entre $q_{1}, q_{2}$ e $q_{3}$ com o menor e com o maior bp-número, respectivamente. Seja $q_{j}$ um vértice em $\left\{q_{1}, q_{2}, q_{3}\right\}$ que é descendente de $m$ e sejam $q_{k}$ e $q_{l}$ vértices distintos em $\left\{q_{1}, q_{2}, q_{3}\right\}$ tais que $k \neq j$ e $l \neq j$. Falta adicionar a obstruction_edges os arcos devidos aos vértices $q_{1}, q_{2}$ e $q_{3}$. Isto é feito da seguinte maneira.

Para $i=1,2,3$, se $q_{i}$ é um terminal, então sejam $u_{1}$ e $u_{2}$ os vértices apontados por neighbor_w_terminall $\left.q_{i}\right]$ e por node_label_b $\left[q_{i}\right]$, respectivamente. A função obstr_edges_terminal (figura 6.12) adiciona os $\operatorname{arcos} u_{1} w$ e $u_{2} w_{i}$ à lista obstruction_edges, além de adicionar os caminhos em $T_{0}$ de $u_{1}$ a $q_{i}$ e de $u_{2}$ a $q_{i}$ nessa lista. Além disso, se $\bar{i}=k$ ou $i=l$ então adiciona-se o caminho em $T_{0}$ de $q_{i}$ a $m^{\prime}$ à lista de arcos. Caso contrário, $i=j$ e adiciona-se o caminho em $T_{0}$ de $q_{j}$ a $m$ à lista de arcos.

Se $q_{i}$ não é um terminal, então $q_{i}=v$. Seja $u$ o vértice apontado por node_label_b $[v]$ e seja $s$ um vértice tal que $s$ coincide com $v$ se $v$ é um descendente de $m$ em $T_{0}$, e $s$ coincide com $w$ caso contrário.

Se $v$ é um descendente de $m$ em $T_{0}$, então $v$ e $m$ são irmãos em $T$ e estão em $\operatorname{RBC}(d)$, para algum C-nó ativo $d$ (figura 6.21), e este é o único caso especial em que a função obstruction_edges_To precisa escolher um dos lados do ciclo facial externo de um C-nó: adiciona-se à 
lista obstruction_edges os arcos que estão no lado do ciclo facial externo de $d$ determinado por $v$ e por $m$ que contém o pai de $d$. Além disso, adiciona-se à lista de arcos da obstrução os arcos no caminho do pai de $d$ a $w$ em $T_{0}$.

Finalmente, se $q_{i}=v$, a função obstr_edges_pnode (figura 6.11) adiciona à lista obstruction_edges o arco $u w_{i}$ e o caminho de $u$ a $s$ em $T_{0}$.

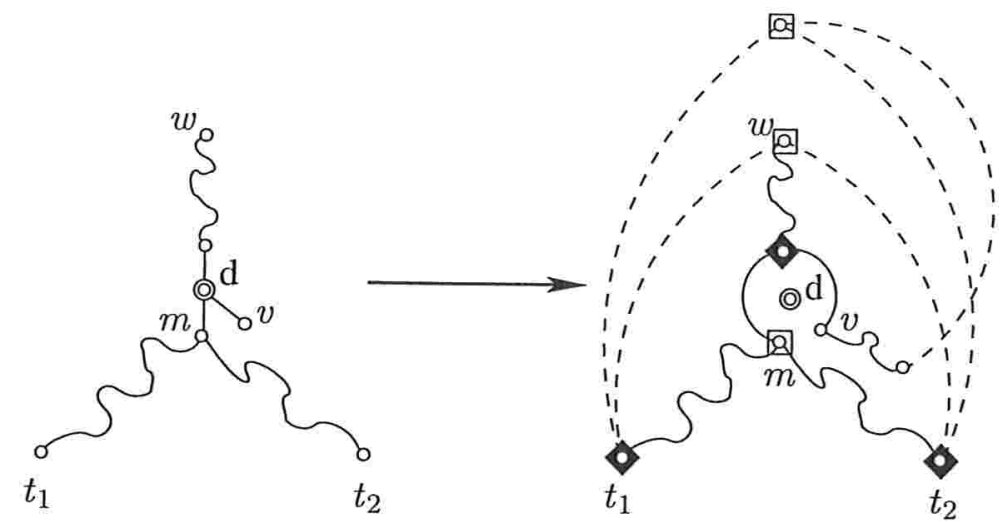

Figura 6.21: Na figura 6.20(a), se $v$ é descendente de $m$ em $T_{0}$ então $v$ e $m$ estão em $\operatorname{RBC}(d)$, para um C-nó ativo $d$, e adiciona-se à lista obstruction_edges os arcos que estão no lado do ciclo facial externo de $d$ determinado por $v$ e por $m$ que contém o pai de $d$.

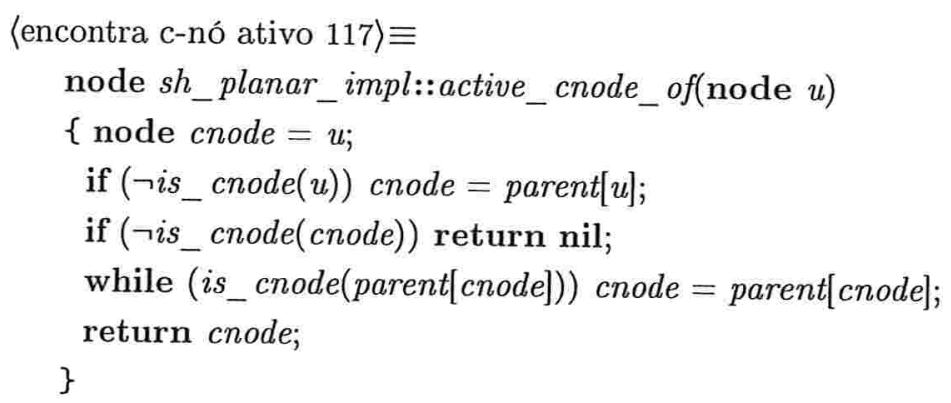

Figura 6.22: Código da função active_cnode_of.

\subsection{Correção}

Observe que os caminhos em $T_{0}$ determinados por cada função que calcula uma lista de arcos de uma obstrução são internamente disjuntos entre si. Além disso, para cada função que inclui o ciclo facial externo $C$ (ou parte dele) de um C-nó na lista obstruction_edges, cada caminho em $T_{0}$ calculado pela função é internamente disjunto de $C$. Disso decorre facilmente a correção desta parte do algoritmo. 


\subsection{Eficiência}

Cada função apresentada neste capítulo executa, basicamente, três tarefas para o cálculo da lista obstruction_edges: cálculo de um número constante de caminhos em $T_{0}$; cálculo de no máximo um ciclo facial externo de um C-nó; e procura por um número constante de arcos (no máximo seis arcos que representam uma aresta de retorno em $T$, com uma ponta em $w$ ou em ancestrais de $w$ ).

A adição de arcos em caminhos em $T_{0}$ à lista obstruction_edges é feita pela função list_edges_upward_TO (figura 6.4) e o tempo gasto por essa função é proporcional ao comprimento do caminho. Como cada caminho em $T_{0}$ tem comprimento máximo $n-1$, onde $n$ é o número total de vértices no grafo, então esta tarefa consome tempo $O(n)$.

Para o cálculo do ciclo facial externo de um C-nó $c$, feito pela rotina extract_boundary_cycle (figura 6.5)), percorre-se no máximo duas vezes o número total de arcos no bloco em $\bar{H}+w$ representado por $c$. Se a função add_part_of_bc (figura 6.16) é executada, então percorre-se no máximo mais uma vez o número total de arcos no bloco em $H+w$ representado por $c$. Como $B_{c}$ é planar, então o tempo consumido por esta tarefa é $O(n)$.

Finalmente, para encontrar um arco no grafo, usa-se a função find_edge (figura 6.3), que percorre a lista de adjacência da ponta inicial do arco, consumindo assim tempo $O(n)$. Logo, totalizando o tempo gasto pelas três tarefas, tem-se que o tempo consumido por cada uma das funções que calcula uma lista de arcos de um obstrução é $O(n)$. 


\section{Resultados experimentais}

Neste capítulo são mostrados alguns resultados experimentais obtidos da comparação de tempos de execução entre algumas implementações para o problema da planaridade: as implementações de Boyer e Myrvold (BM), de Shih e Hsu (SH) e as duas implementações presentes no LEDA: uma implementação de Booth e Lueker (BL) para o algoritmo de LEC e uma implementação de Hopcroft e Tarjan (HT) para o algoritmo de Auslander, Parter e Goldstein.

Lembre-se de que o problema da planaridade consiste em decidir se um dado grafo é ou não é planar e justificar a resposta com um certificado, que neste caso é ou uma descrição combinatória plana ou um subgrafo de Kuratowski do grafo. Dentre as implementações citadas acima, apenas HT não justifica a resposta para grafos não-planares.

Os dados presentes neste capítulo foram obtidos por uma versão modificada do programa planarity_time, que é um programa de demonstração que integra o pacote do LEDA. Basicamente, foram adicionadas as implementações de $\mathrm{BM}$ e de $\mathrm{SH}$ ao programa original.

Durante os testes realizados, a implementação de BM foi a que apresentou o melhor desempenho, mas apenas esta implementação, dentre as quatro, não foi desenvolvida sobre a plataforma LEDA.

Além disso, o programa planarity_time modificado converte o formato de entrada do LEDA para o formato de entrada da implementação de BM, mas a saída construída por BM não foi convertida para o formato LEDA, pois seria necessário mais tempo para estudar e mudar o seu código (os rótulos dos vértices no grafo de entrada são modificados durante a execução de BM). Por esse mesmo motivo, como o código original de BM foi escrito especificamente para resolver o problema da planaridade, não foi medido o tempo gasto pela implementação de BM para testar planaridade sem a construção de um certificado para a resposta. 


\subsection{Ambiente experimental}

Os testes foram rodados num PC com sistema GNU/Linux (RedHat 7.1), com um processador Celeron de $700 \mathrm{MHz}$ e $256 \mathrm{MB}$ de memória RAM. O programa de testes foi compilado com o gcc versão 2.96, com as opções de compilação -DLEDA_CHECKING_OFF -0 e com o LEDA versão 4.4.

As instâncias usadas nos testes foram os mesmos "tipos" de grafos (descritos mais adiante) usados no programa original planarity_time. Em todos os testes, para cada valor de $n$, onde $n$ é o número de vértices do grafo, foi obtida a média entre os valores de tempo relativos a dez grafos. Cada valor de tempo foi medido pela função used_time do LEDA, cujo código é ilustrado na figura 7.1.

$\langle$ tempo de uso de CPU 120〉三

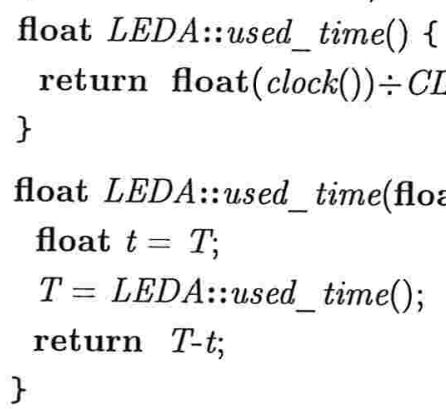

Figura 7.1: Código da função used_time do LEDA versão 4.2.

Os tipos de grafos usados nos testes foram os seguintes:

(G1) grafos planares aleatórios;

(G2) grafos com um $K_{3,3}$. Cada grafo do tipo (G2) é obtido da seguinte maneira. Seis vértices de um grafo planar aleatório $G$ são escolhidos para a construção de um $K_{3,3}$ em $G$, adicionandose arestas a $G$;

(G3) grafos com um $K_{5}$. Cada grafo do tipo (G3) é obtido da seguinte maneira. Cinco vértices de um grafo planar aleatório $G$ são escolhidos para a construção de um $K_{5}$ em $G$, adicionandose arestas a $G$;

(G4) grafos planares maximais;

(G5) grafos planares maximais a menos de uma aresta. Cada grafo do tipo (G5) é obtido da seguinte maneira. Seja $G$ um grafo planar maximal e sejam $u$ e $v$ dois vértices de $G$ tais que $u v$ não está em $E_{G}$. O grafo $G+u v$ é um grafo do tipo (G5). 
As funções usadas para gerar grafos planares maximais e grafos aleatórios planares usam as funções maximal_planar_map (figura 7.2) e random_planar_map (figura 7.3), respectivamente. A primeira gera um grafo planar maximal com $n$ vértices e a segunda gera um grafo planar com $n$ vértices e $m \leq 3 n-6$ arestas. (O reverso de um arco $e$ num grafo $G$ é acessado pela função G.reversal(e) e o sucessor cíclico de $e$, no ciclo facial que contém $e$, é acessado pela função G.face_cycle_succ(e).)

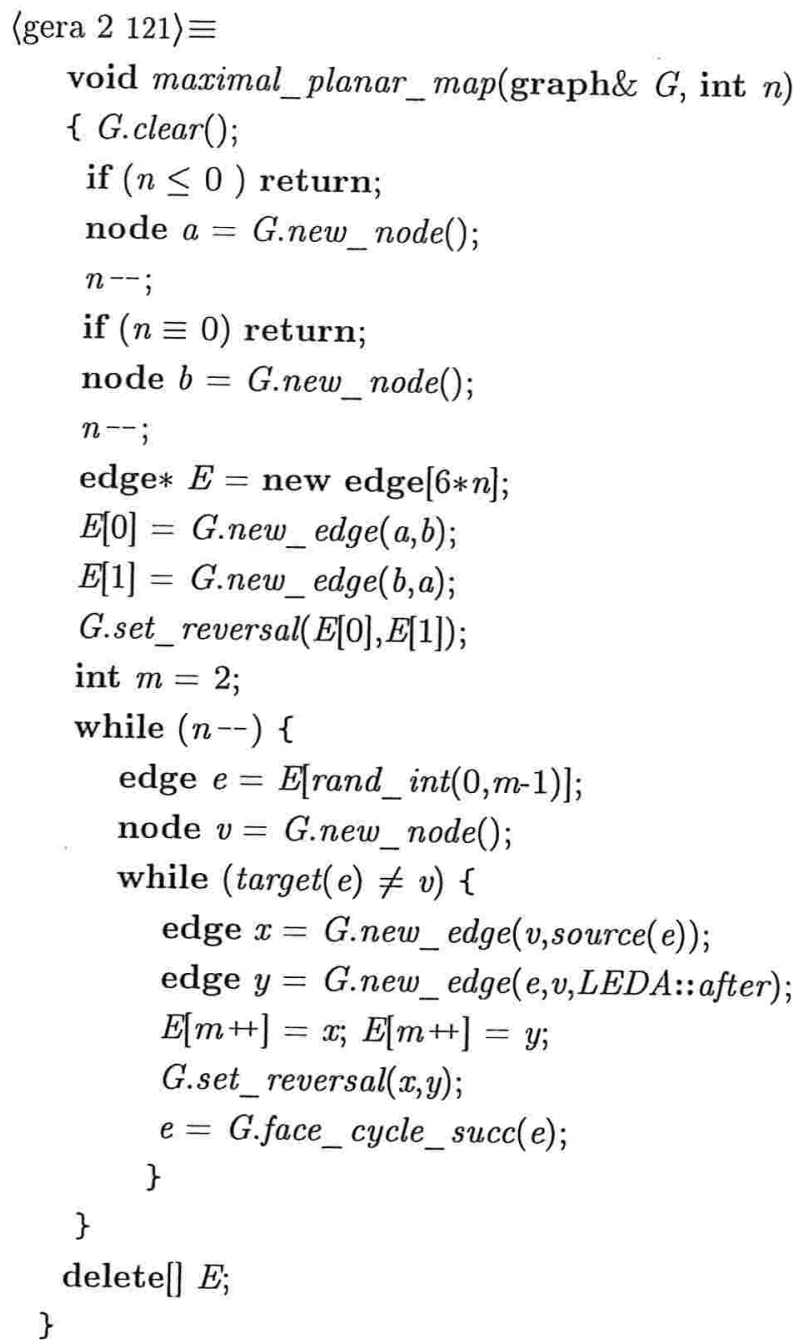

Figura 7.2: Código da função maximal_planar_map do LEDA versão 4.2.

A tabela com os tempos de execução de cada implementação é ilustrada na figura 7.5. Nela, não foram medidos os tempos de teste de planaridade sem o cálculo do certificado para a implementação de BM, onde a coluna T foi substituída pela coluna TJ.

A seguir, para cada tipo de grafo usado nos testes, encontram-se uma descrição de como os grafos foram gerados, uma tabela do consumo de espaço para cada uma das implementações e dois 


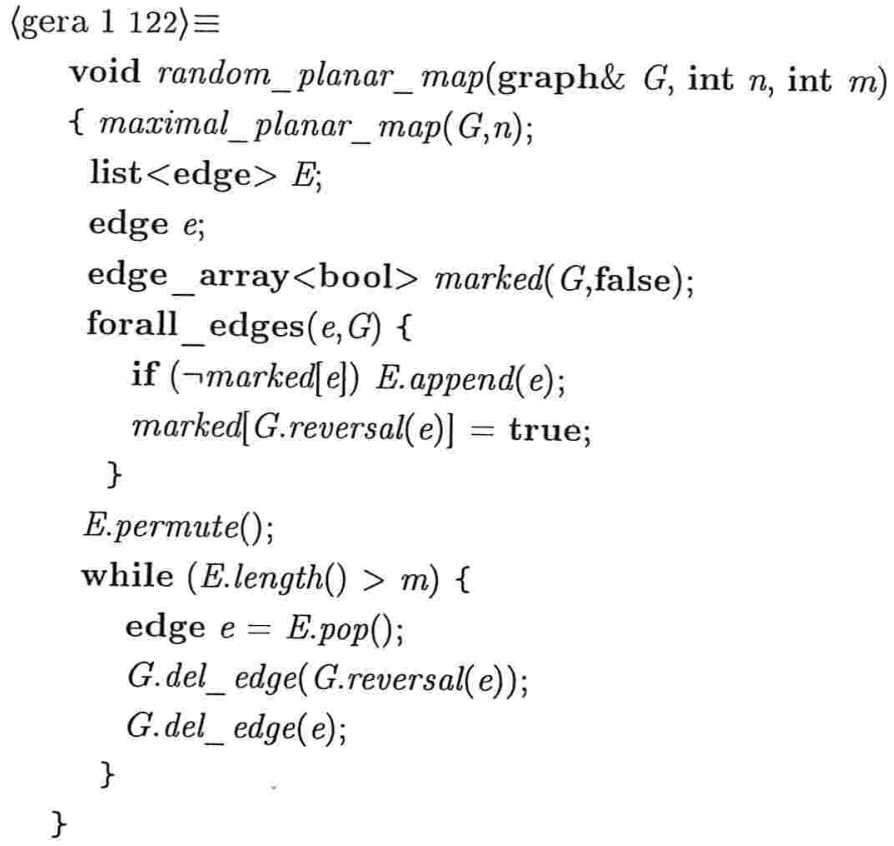

Figura 7.3: Código da função random_planar_map do LEDA versão 4.2.

gráficos obtidos com os dados da figura 7.5, um para o teste sem cálculo do certificado e outro para o teste com construção do certificado e verificação deste. (Na implementação de SH a verificação de uma descrição combinatória plana é feita pela função check_embedding na seção 2.5.)

O espaço consumido foi medido pela função print_statistics do LEDA, que mostra um resumo da memória usada como mostrado na figura 7.4.

STD_MEMORY_MGR (memory status)
\begin{tabular}{|rrrrr|}
\hline size & used & free & blocks & bytes \\
\hline 8 & 0 & 1022 & 1 & 8176 \\
12 & 0 & 20430 & 30 & 245160 \\
44 & 0 & 8325 & 45 & 366300 \\
52 & 0 & 2028 & 13 & 105456 \\
64 & 0 & 630 & 5 & 40320 \\
time: $0.20 \mathrm{sec}$ & \multicolumn{3}{c}{ space: $751.63 \mathrm{~kb}$} \\
\hline
\end{tabular}

Figura 7.4: Saída da função print_statistics do LEDA, que mostra um resumo da memória usada. 


\begin{tabular}{|c|c|c|c|c|c|c|c|c|c|}
\hline & & \multicolumn{2}{|c|}{$\mathrm{BL}$} & \multicolumn{2}{|c|}{$\mathrm{HT}$} & \multicolumn{2}{|c|}{$\mathrm{BM}$} & \multicolumn{2}{|c|}{$\mathrm{SH}$} \\
\hline Tipo & $n$ & $T$ & $T J C$ & $T$ & $T J C$ & $T J$ & $T J C$ & $T$ & $T J C$ \\
\hline \multirow[t]{7}{*}{ (G1) } & 1000 & 0.03 & 0.06 & 0.09 & 0.13 & 0.02 & 0.02 & 0.12 & 0.77 \\
\hline & 2000 & 0.06 & 0.13 & 0.19 & 0.30 & 0.05 & 0.06 & 0.28 & 1.65 \\
\hline & 4000 & 0.13 & 0.29 & 0.43 & 0.69 & 0.12 & 0.13 & 0.60 & 3.38 \\
\hline & 8000 & 0.27 & 0.60 & 0.89 & 1.49 & 0.24 & 0.27 & 1.26 & 6.88 \\
\hline & 16000 & 0.57 & 1.23 & 1.87 & 3.19 & 0.50 & 0.56 & 2.63 & 13.99 \\
\hline & 32000 & 1.20 & 2.59 & 3.95 & 6.93 & 1.08 & 1.19 & 5.45 & 28.70 \\
\hline & 64000 & 2.55 & 5.52 & 8.40 & 15.13 & 2.22 & 2.40 & 11.58 & 58.07 \\
\hline \multirow[t]{7}{*}{ (G2) } & 1000 & 0.02 & 0.22 & 0.11 & - & 0.02 & 0.03 & 0.11 & 0.21 \\
\hline & 2000 & 0.05 & 0.46 & 0.23 & - & 0.06 & 0.06 & 0.24 & 0.47 \\
\hline & 4000 & 0.10 & 0.94 & 0.48 & - & 0.13 & 0.13 & 0.43 & 0.75 \\
\hline & 8000 & 0.20 & 1.86 & 0.99 & - & 0.27 & 0.29 & 1.04 & 2.09 \\
\hline & 16000 & 0.42 & 3.93 & 2.03 & - & 0.57 & 0.61 & 1.82 & 3.40 \\
\hline & 32000 & 0.83 & 7.81 & 4.24 & - & 1.04 & 1.10 & 3.94 & 7.45 \\
\hline & 64000 & 1.79 & 16.86 & 8.62 & - & 2.24 & 2.39 & 8.01 & 15.30 \\
\hline \multirow[t]{7}{*}{ (G3) } & 1000 & 0.03 & 0.24 & 0.12 & - & 0.02 & 0.02 & 0.10 & 0.19 \\
\hline & 2000 & 0.05 & 0.52 & 0.25 & - & 0.06 & 0.06 & 0.25 & 0.48 \\
\hline & 4000 & 0.11 & 1.02 & 0.52 & - & 0.13 & 0.14 & 0.47 & 0.89 \\
\hline & 8000 & 0.22 & 2.03 & 1.05 & - & 0.27 & 0.29 & 1.05 & 2.04 \\
\hline & 16000 & 0.45 & 4.23 & 2.11 & - & 0.50 & 0.55 & 1.84 & 3.21 \\
\hline & 32000 & 0.91 & 8.71 & 4.38 & - & 1.06 & 1.12 & 3.89 & 7.15 \\
\hline & 64000 & 1.95 & 18.19 & 8.92 & - & 2.16 & 2.31 & 8.29 & 15.48 \\
\hline \multirow[t]{7}{*}{ (G4) } & 1000 & 0.05 & 0.11 & 0.18 & 0.28 & 0.03 & 0.03 & 0.25 & 1.52 \\
\hline & 2000 & 0.10 & 0.22 & 0.37 & 0.59 & 0.07 & 0.08 & 0.51 & 3.08 \\
\hline & 4000 & 0.21 & 0.44 & 0.73 & 1.21 & 0.16 & 0.17 & 1.02 & 5.94 \\
\hline & 8000 & 0.42 & 0.88 & 1.46 & 2.48 & 0.30 & 0.34 & 2.03 & 12.21 \\
\hline & 16000 & 0.85 & 1.76 & 2.95 & 5.16 & 0.65 & 0.68 & 4.12 & 23.05 \\
\hline & 32000 & 1.72 & 3.59 & 6.19 & 11.00 & 1.28 & 1.41 & 8.54 & 48.58 \\
\hline & 64000 & 3.61 & 7.62 & 12.48 & 23.15 & 2.64 & 2.99 & 18.24 & 94.94 \\
\hline \multirow[t]{7}{*}{ (G5) } & 1000 & 0.04 & 0.33 & 0.08 & - & 0.03 & 0.03 & 0.21 & 0.43 \\
\hline & 2000 & 0.07 & 0.62 & 0.16 & - & 0.07 & 0.08 & 0.40 & 0.86 \\
\hline & 4000 & 0.14 & 1.18 & 0.33 & - & 0.17 & 0.18 & 0.85 & 1.88 \\
\hline & 8000 & 0.34 & 2.78 & 0.66 & - & 0.34 & 0.36 & 1.77 & 4.28 \\
\hline & 16000 & 0.57 & 4.79 & 1.39 & - & 0.67 & 0.72 & 3.10 & 6.74 \\
\hline & 32000 & 1.24 & 10.26 & 2.78 & - & 1.35 & 1.48 & 6.78 & 15.49 \\
\hline & 64000 & 2.71 & 21.69 & 5.83 & - & 2.76 & 2.94 & 14.59 & 33.40 \\
\hline
\end{tabular}

Figura 7.5: Na tabela acima, encontram-se os tempos medidos em segundos para cada uma das implementações: $\mathrm{BL}, \mathrm{HT}, \mathrm{BM}$ e SH. Para cada valor de $n$, onde $n$ é o número de vértices, cada valor é a média entre dez medidas de tempo e, para os tipos (G1), (G2) e (G3), usou-se $m=2 n$ arestas. A coluna $\mathrm{T}$ representa o tempo gasto para testar planaridade sem justificar o resultado. Já as colunas TJ e TJC representam os tempos gastos para testar e obter um certificado. A diferença entre estes dois é que TJC inclui o tempo gasto para verificar o certificado. 


\subsection{Grafos planares aleatórios (G1)}

Para gerar grafos aleatórios planares, usou-se a função random_planar_graph do LEDA (figura 7.6), que recebe como parâmetros uma variável $G$ do tipo graph e inteiros $n$ e $m$, que são, respectivamente, o número de vértices e o número de arestas do grafo $G$ a ser gerado. Para os testes, usou-se $m=2 n$.

〈gera grafos aleatórios planares 124$\rangle \equiv$

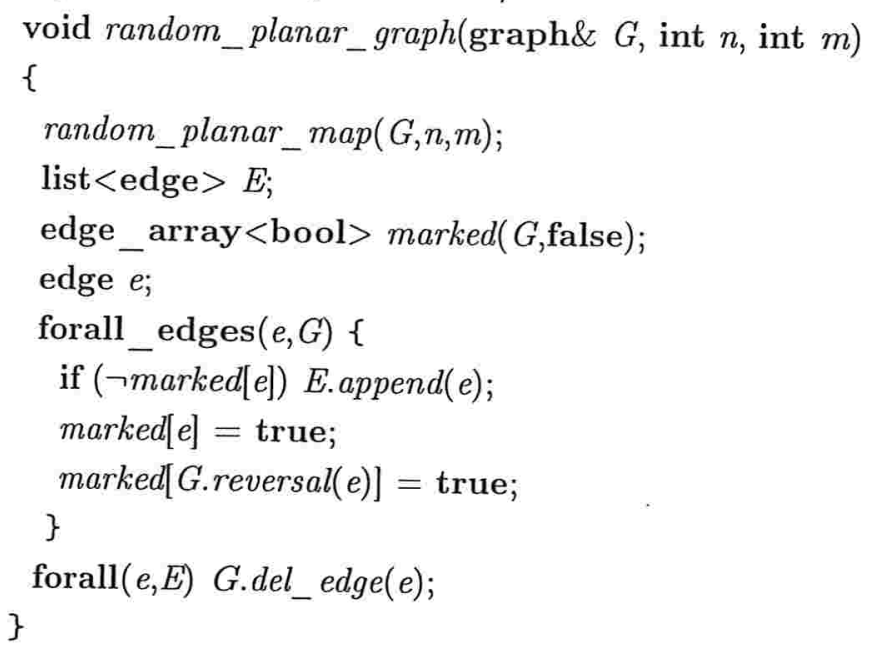

Figura 7.6: Código da função random_planar_graph do LEDA.

Na figura 7.7 encontra-se uma tabela com as medidas do espaço consumido por cada implementação para grafos do tipo (G1). Neste caso, observou-se que HT obteve o pior desempenho.

\begin{tabular}{lrrrr}
\hline \hline$n$ & BL & HT & BM & SH \\
\hline \hline 1000 & 751.63 & 1195.43 & 671.67 & 815.60 \\
\hline 2000 & 1487.27 & 2382.85 & 1327.35 & 1615.21 \\
\hline 4000 & 2886.59 & 4725.71 & 2646.71 & 3206.43 \\
\hline 8000 & 5725.20 & 9443.41 & 5285.42 & 6388.88 \\
\hline 16000 & 11362.45 & 18854.83 & 10562.84 & 12721.79 \\
\hline 32000 & 22652.93 & 37701.65 & 21117.68 & 25443.57 \\
\hline 64000 & 45042.00 & 75339.33 & 42227.37 & 50831.17 \\
\hline \hline
\end{tabular}

Figura 7.7: Espaço consumido para grafos planares aleatórios (G1) com $n$ vértices. Para cada valor de $n$ foi medido o espaço gasto por cada algoritmo, em Kbytes.

Os gráficos com os dados da tabela 7.5 para grafos aleatórios planares são ilustrados na figura 7.8. Nos dois gráficos, observa-se que as implementações de BM e de BL obtiveram os melhores desempenhos. 
(G1) TESTE

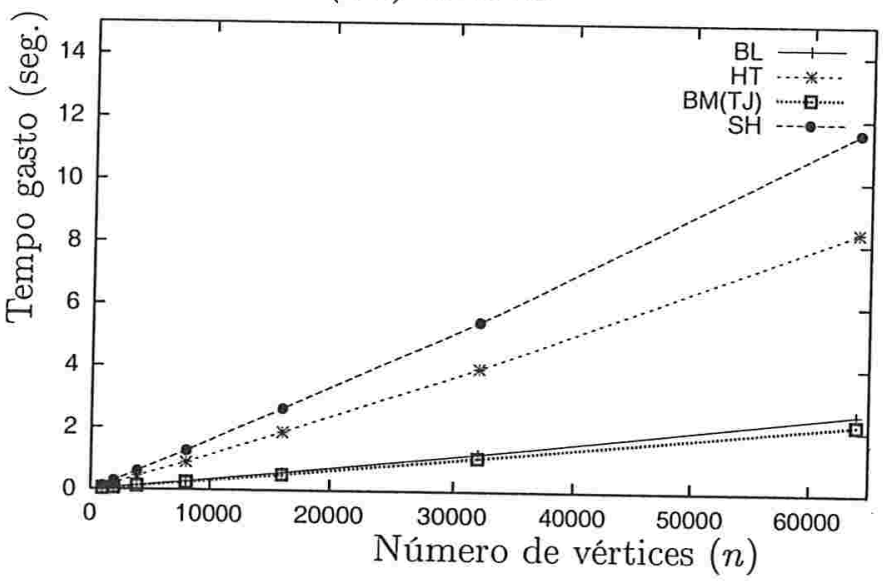

(G1) TESTE + CERTIFICADO

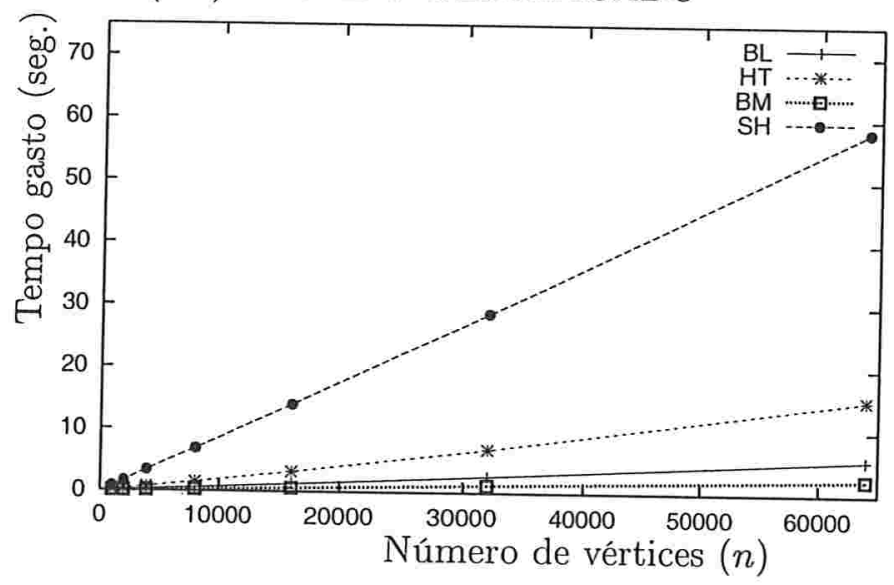

Figura 7.8: Tempos gastos pelas implementações com grafos planares aleatórios (G1). O primeiro gráfico ilustra o tempo para apenas testar planaridade. Neste, apenas BM executa o cálculo do certificado. O segundo ilustra o tempo gasto para testar, justificar e verificar o certificado construído. 


\subsection{Grafos não-planares do tipo (G2)}

Os grafos não-planares do tipo (G2) foram gerados conforme mostrado na figura 7.9. Primeiramente um grafo planar $G$ com $n$ vértices e $m=2 n$ arestas é gerado aleatoriamente. Em seguida, seis vértices de $G$ são escolhidos para fazer parte de um $K_{3,3}$ em $G$, construído adicionando-se arestas a $G$.

$\langle$ gera grafos aleatórios planares + K33 126〉三

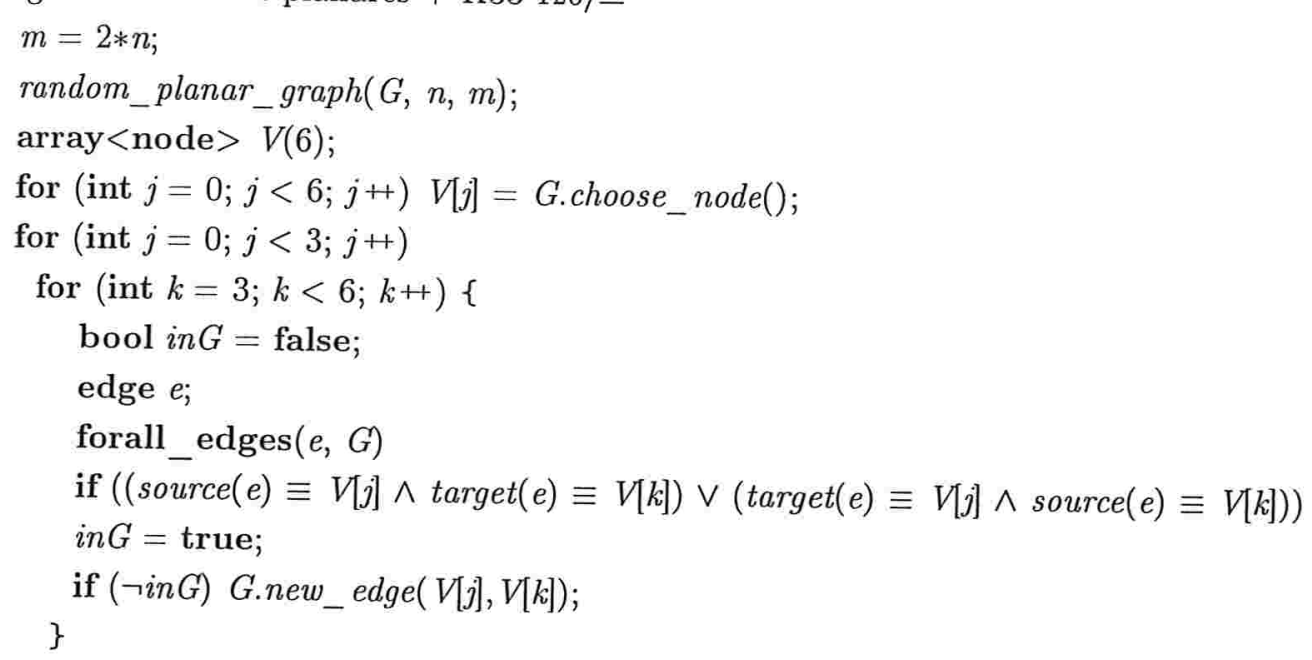

Figura 7.9: Trecho de código (do LEDA) para gerar grafos não-planares do tipo (G2).

Na figura 7.10 encontra-se uma tabela com as medidas do espaço consumido por cada implementação para grafos do tipo (G2). Neste caso, observou-se que HT e BL consumiram mais espaço.

\begin{tabular}{lrrrr}
\hline \hline$n$ & BL & HT & BM & SH \\
\hline \hline 1000 & 1143.44 & 1171.44 & 671.67 & 687.66 \\
\hline 2000 & 2047.00 & 2310.88 & 1327.35 & 1359.34 \\
\hline 4000 & 4078.01 & 4597.77 & 2654.70 & 2678.69 \\
\hline 8000 & 8715.74 & 9179.54 & 5293.41 & 5357.38 \\
\hline 16000 & 16168.10 & 18295.10 & 10578.83 & 10642.80 \\
\hline 32000 & 32200.27 & 36574.20 & 21117.68 & 21589.45 \\
\hline 64000 & 62993.23 & 73300.33 & 42227.37 & 42595.19 \\
\hline \hline
\end{tabular}

Figura 7.10: Espaço consumido para grafos do tipo (G2) com $n$ vértices. Para cada valor de $n$ foi medido o espaço gasto por cada algoritmo, em Kbytes.

Os gráficos com os dados da tabela 7.5 para grafos do tipo (G2) são ilustrados na figura 7.11. No primeiro, apesar de BL ter apresentado o melhor desempenho, juntamente com BM, BL 
apresentou o pior desempenho no segundo, juntamente com SH. Como a implementação de HT não calcula o certificado para grafos não-planares, esta foi omitida do segundo gráfico.

(G2) TESTE

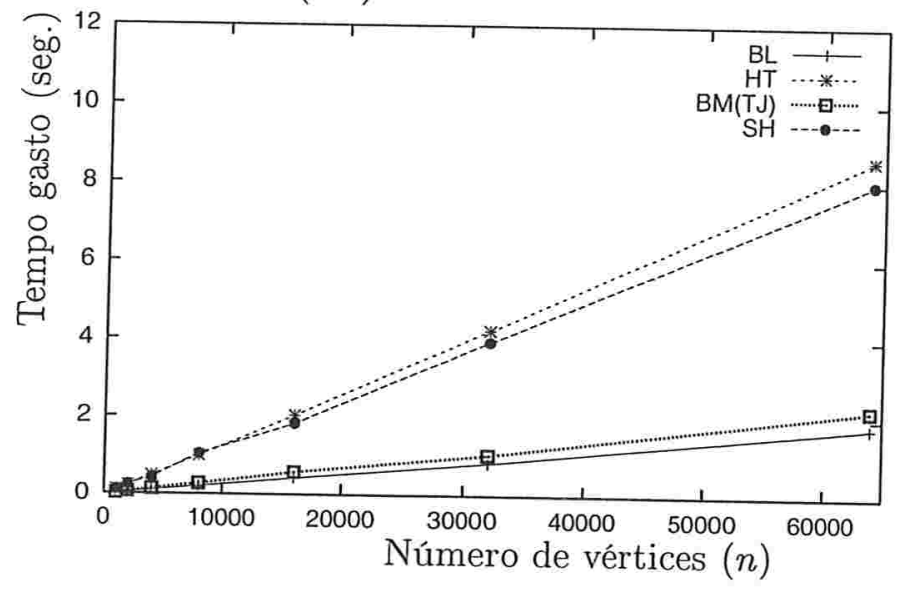

(G2) TESTE + CERTIFICADO

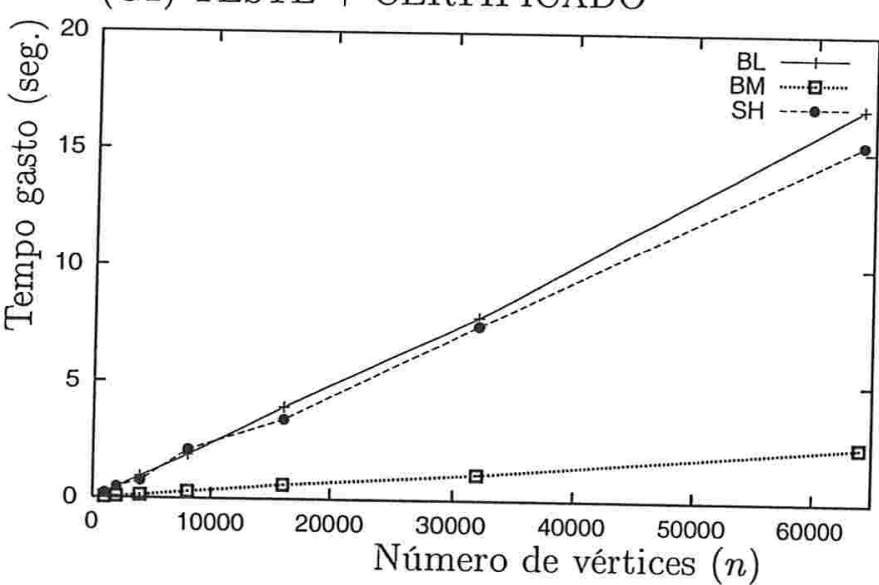

Figura 7.11: Tempos gastos pelas implementações com grafos não-planares do tipo (G2). O primeiro gráfico ilustra o tempo gasto para apenas testar planaridade. Neste, apenas BM executa o cálculo do certificado. O segundo ilustra o tempo gasto para testar, justificar e verificar o certificado construído. 


\subsection{Grafos não-planares do tipo (G3)}

Os grafos não-planares do tipo (G3) foram gerados conforme mostrado na figura 7.12. Primeiramente um grafo planar $G$ com $n$ vértices e $m=2 n$ arestas é gerado aleatoriamente. Em seguida, cinco vértices de $G$ são escolhidos para fazer parte de um $K_{5}$ em $G$, construído adicionando-se arestas a $G$.

〈gera grafos aleatórios planares + K5 128) $\equiv$

$m=2 * n ;$

random_planar_graph $(G, n, m)$;

array $<$ node $>V(5)$;

for (int $j=0 ; j<5 ; j+$ )

for (int $k=j+1 ; k<5 ; k+$ ) \{

bool in $G=$ false;

edge $e$;

forall_edges $(e, G)$

if $((\operatorname{source}(e) \equiv V[j] \wedge \operatorname{target}(e) \equiv V[k]) \vee(\operatorname{target}(e) \equiv V[j] \wedge \operatorname{source}(e) \equiv V[k]))$ in $G=$ true;

if $(\neg i n G)$ G.new_edge $(V[j], V[k])$;

\}

Figura 7.12: Trecho de código (do LEDA) para gerar grafos não-planares do tipo (G3).

Na figura 7.13 encontra-se uma tabela com as medidas do espaço consumido para grafos do tipo (G3). Neste caso, observou-se novamente que HT e BL consumiram mais espaço.

\begin{tabular}{lrrrr}
\hline \hline$n$ & BL & HT & BM & SH \\
\hline \hline 1000 & 1103.46 & 1171.44 & 671.67 & 687.66 \\
\hline 2000 & 2023.01 & 2310.88 & 1327.35 & 1359.34 \\
\hline 4000 & 3910.09 & 4597.77 & 2654.70 & 2702.68 \\
\hline 8000 & 7972.11 & 9171.54 & 5293.41 & 5397.36 \\
\hline 16000 & 16583.90 & 18295.10 & 10578.83 & 10762.74 \\
\hline 32000 & 33983.40 & 36574.20 & 21117.68 & 21245.62 \\
\hline 64000 & 62961.24 & 73300.33 & 42227.37 & 42715.13 \\
\hline \hline
\end{tabular}

Figura 7.13: Espaço consumido para grafos do tipo (G3) com $n$ vértices. Para cada valor de $n$ foi medido o espaço gasto por cada algoritmo, em Kbytes.

Os gráficos com os dados da tabela 7.5 para grafos do tipo (G3) são ilustrados na figura 7.14. Como observado na seção anterior, BL e BM apresentaram um bom desempenho no primeiro gráfico. Já no segundo, BL e SH apresentaram os piores desempenhos. 
(G3) TESTE

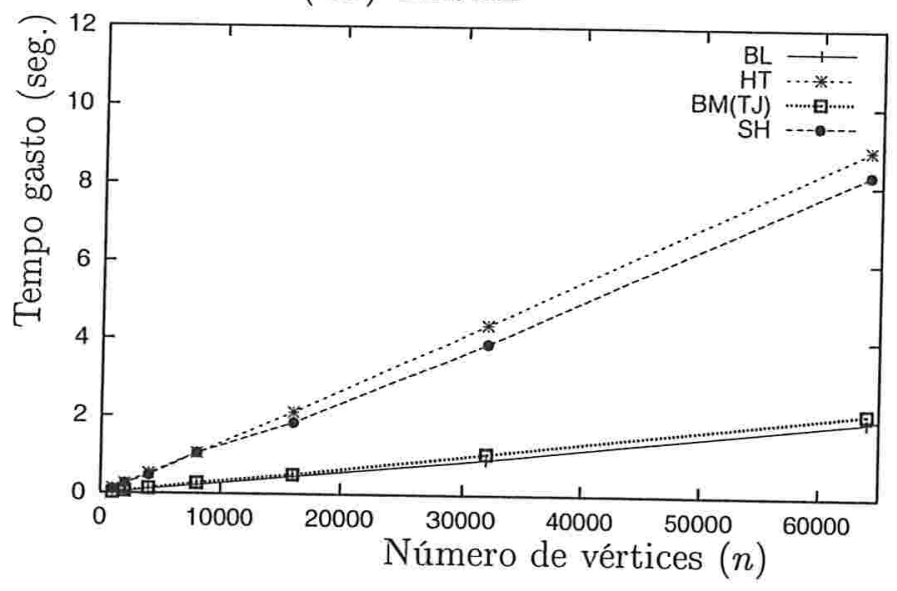

(G3) TESTE + CERTIFICADO

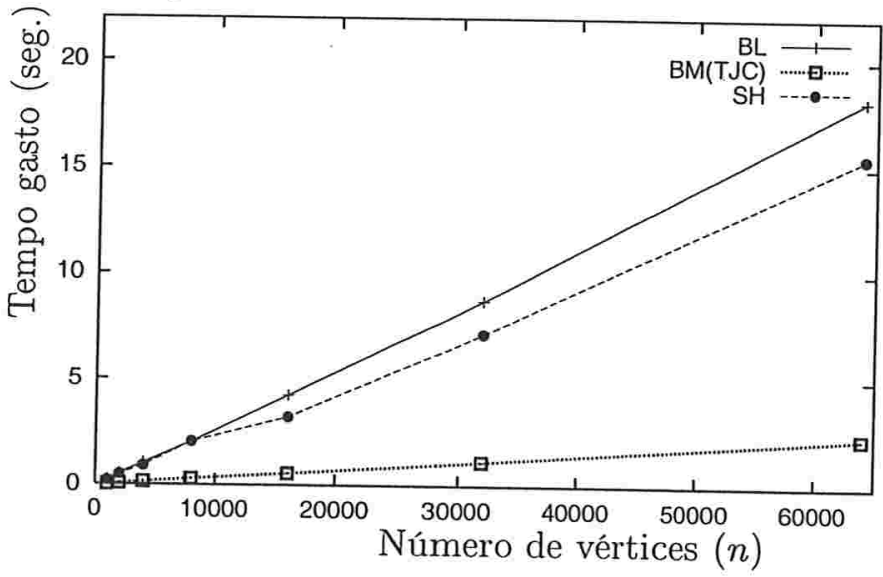

Figura 7.14: Tempos gastos pelas implementações com grafos não-planares do tipo (G3). O primeiro gráfico ilustra o tempo gasto para apenas testar planaridade. Neste, apenas BM executa o cálculo do certificado. O segundo ilustra o tempo gasto para testar, justificar e verificar o certificado construído. 


\subsection{Grafos planares maximais (G4)}

Para gerar grafos maximais planares, usou-se a função maximal_planar_graph do LEDA (figura 7.15), que recebe como parâmetros uma variável $G$ do tipo graph e um inteiro $n$ representando o número de vértices do grafo a ser gerado.

$\langle$ gera grafos maximais planares 130$\rangle \equiv$

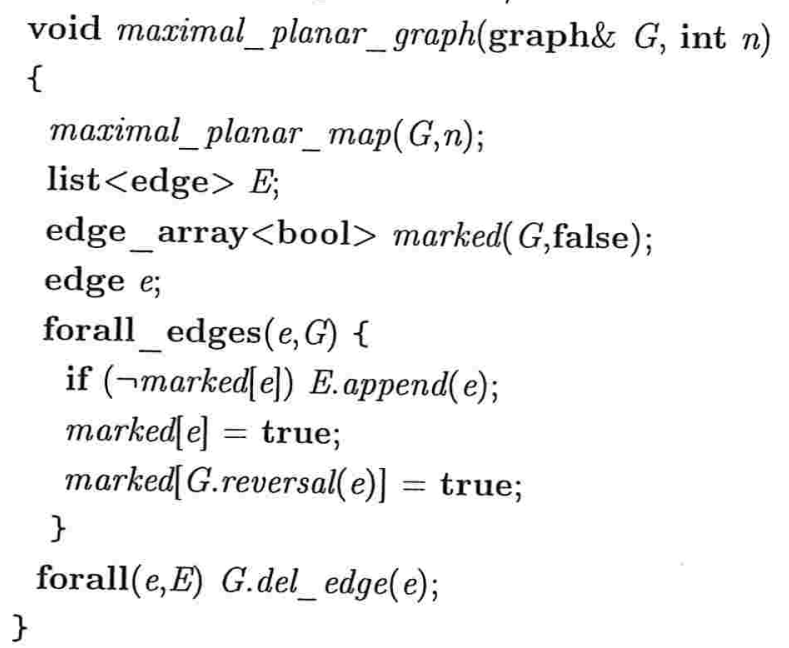

Figura 7.15: Código da rotina maximal_planar_graph do LEDA.

A tabela da figura 7.16 mostra as medidas do espaço consumido para grafos planares maximais. Neste caso, observou-se que HT foi a implementação que consumiu mais espaço.

\begin{tabular}{lrrrr}
\hline \hline$n$ & $\cdot$ BL & HT & BM & SH \\
\hline \hline 1000 & 1023.50 & 1651.20 & 943.54 & 1111.46 \\
\hline 2000 & 1991.03 & 3286.41 & 1887.08 & 2198.93 \\
\hline 4000 & 3950.07 & 6532.82 & 3766.16 & 4365.87 \\
\hline 8000 & 7796.19 & 13049.65 & 7516.33 & 8691.75 \\
\hline 16000 & 15552.40 & 26067.30 & 15024.66 & 17383.51 \\
\hline 32000 & 30768.97 & 52102.62 & 30033.33 & 34743.03 \\
\hline 64000 & 61617.90 & 104189.23 & 60058.66 & 69414.09 \\
\hline \hline
\end{tabular}

Figura 7.16: Espaço consumido para grafos planares maximais (G4) com $n$ vértices.

Os gráficos com os dados da tabela 7.5 para grafos planares maximais são ilustrados na figura 7.17. Nos dois gráficos, BL e BM apresentaram os melhores desempenhos e SH foi o pior. 

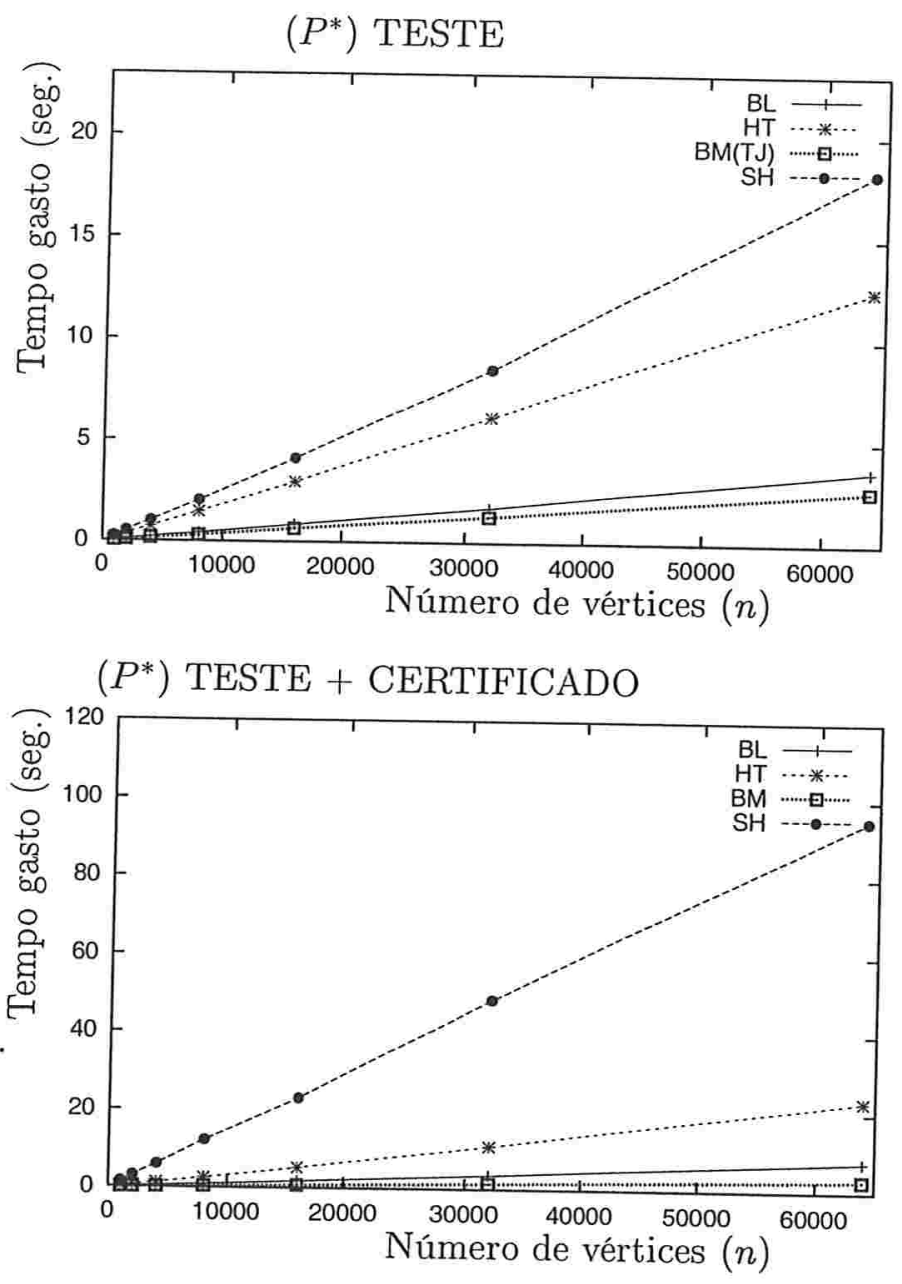

Figura 7.17: Tempos gastos pelas implementações com grafos planares maximais (G4). O primeiro gráfico ilustra o tempo gasto para apenas testar planaridade. Neste, apenas BM executa o cálculo do certificado. O segundo ilustra o tempo gasto para testar, justificar e verificar o certificado construído. 


\subsection{Grafos não-planares do tipo (G5)}

Os grafos não-planares do tipo (G5) usados nos testes foram gerados conforme mostra a figura 7.18. Primeiramente foi gerado um grafo planar maximal $G$ com $n$ vértices e em seguida adicionou-se uma nova aresta a $G$ entre dois vértices não-adjacentes.

〈gera grafos maximais planares mais uma aresta 132$\rangle \equiv$

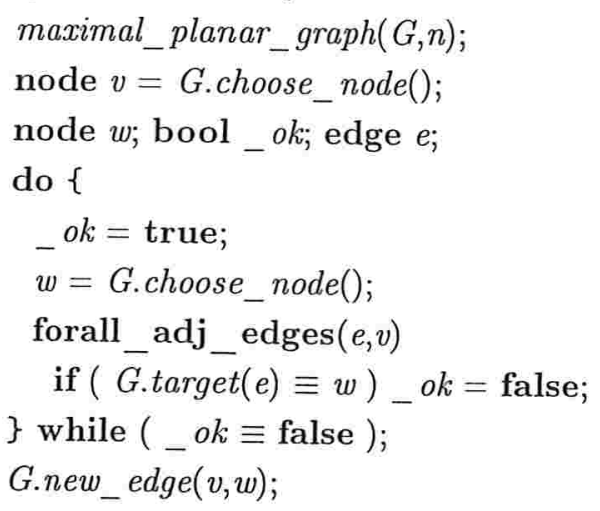

Figura 7.18: Trecho de código para gerar grafos não-planares do tipo (G5).

A tabela da figura 7.19 mostra as medidas do espaço consumido para grafos do tipo (G5). Neste caso, observou-se que BL foi a implementação que consumiu mais espaço.

\begin{tabular}{lrrrr}
\hline \hline$n$ & BL & HT & BM & SH \\
\hline \hline 1000 & 1799.12 & 1267.39 & 943.54 & 975.52 \\
\hline 2000 & 2862.60 & 2518.78 & 1887.08 & 1935.05 \\
\hline 4000 & 6109.02 & 5021.56 & 3766.16 & 3846.12 \\
\hline 8000 & 11354.45 & 10035.12 & 7516.33 & 7732.22 \\
\hline 16000 & 22948.79 & 20054.24 & 15024.66 & 15328.51 \\
\hline 32000 & 47856.62 & 40100.48 & 30033.33 & 30832.94 \\
\hline 64000 & 85574.20 & 80184.96 & 60058.66 & 60866.27 \\
\hline \hline
\end{tabular}

Figura 7.19: Espaço consumido para grafos planares maximais (G5).

Os gráficos com os dados da tabela 7.5 para grafos planares maximais são ilustrados na figura 7.17. Nos dois gráficos, SH obteve o pior desempenho. Como foi observado para grafos não-planares dos tipos (G2) e (G3), BL apresentou o melhor desempenho no primeiro, junto com BM. Já no segundo, BL teve um desempenho ruim. 
(G5) TESTE

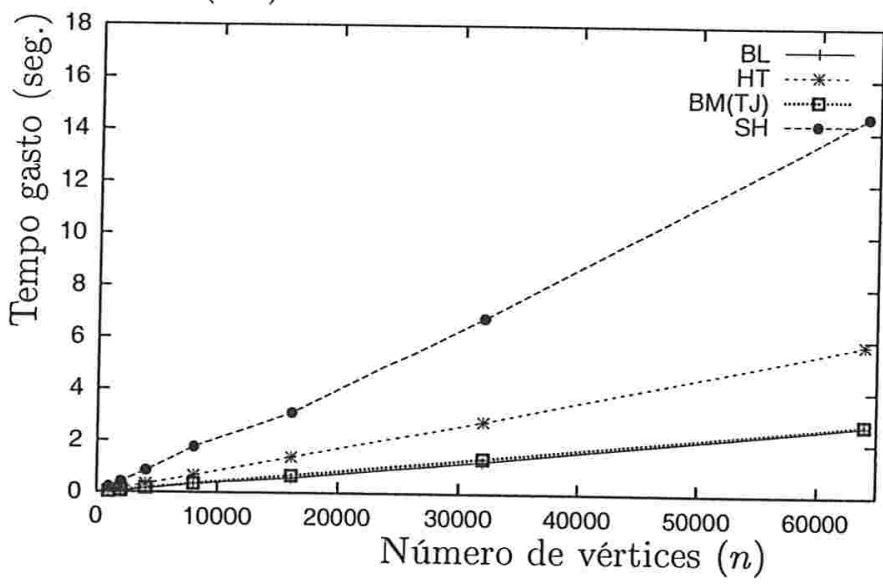

(G5) TESTE + CERTIFICADO

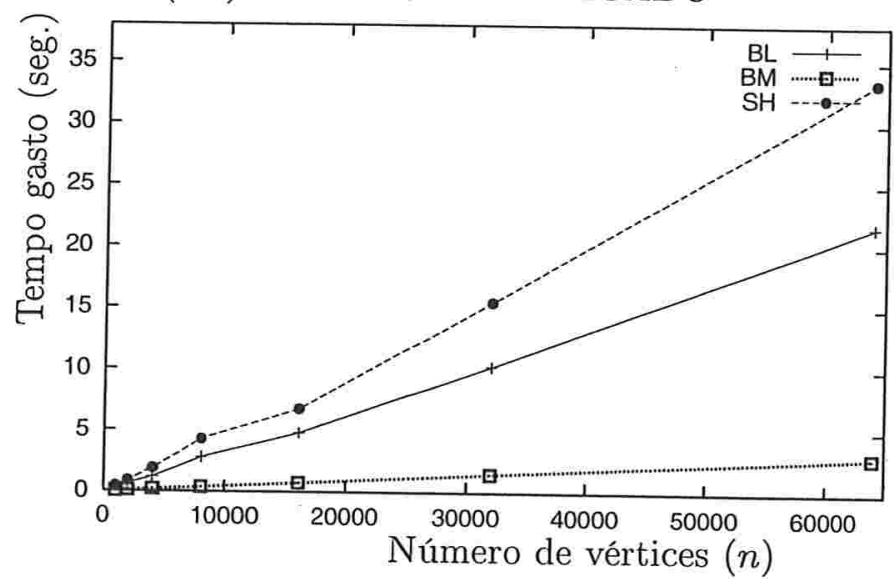

Figura 7.20: Tempos gastos pelas implementações com grafos não-planares do tipo (G5). O primeiro gráfico ilustra o tempo gasto para apenas testar planaridade. Neste, apenas BM executa o cálculo do certificado. O segundo ilustra o tempo gasto para testar, justificar e verificar o certificado construído. 



\section{Comentários finais}

Nesta dissertação, apresentamos uma descrição do algoritmo de LEC e uma implementação deste, proposta por Shih e Hsu. A implementação de SH descrita neste trabalho foi realizada sobre a plataforma LEDA.

Nosso estudo começou pelo artigo publicado por Shih e Hsu [15]. Nele encontra-se uma descrição para resolver o problema do teste de planaridade, mas com alguns erros, conforme mostra Boyer [4]. Além disso, muitos detalhes são omitidos neste artigo. Como exemplos, podemos citar o uso do contador para cada C-nó para testar a presença de uma $X Y$-obstrução do tipo (o1), a existência de C-nós inativos na PC-árvore alterada $T$ e a determinação dos C-nós ativos em $T$.

O desenvolvimento da implementação de SH foi iniciada após o estudo do artigo publicado por Thomas [18]. Através deste artigo conseguimos enxergar o algoritmo de SH "de um nível mais alto" e nos ajudou a desvendar muitos dos detalhes omitidos no primeiro artigo.

Nossa primeira versão da implementação que resolvia o problema do teste de planaridade consumia tempo quadrático no número de vértices do grafo e consistia basicamente de dois passos, além do pré-processamento: um passo para calcular as componentes de $T-L$ e seus respectivos terminais e um outro passo para estender a PC-árvore alterada $T$ para a próxima iteração, caso não existisse uma $X Y$-obstrução. Observe que nessa versão, a busca por uma $X Y$-obstrução e a atualização de $T$ eram feitas em um passo apenas. Apesar de usarmos duas listas duplamente ligadas para representar cada RBC (cada uma num sentido para evitar o custo para inverter a lista), esta versão era quadrática pois C-nós inativos eram percorridos durante os dois passos básicos executados nesta versão.

Numa segunda versão da implementação, aplicamos a heurística union-find [16] para compressão de caminhos formados por C-nós inativos consecutivos. A idéia era comprimir todos os caminhos percorridos em $T$ durante a fase do cálculo dos terminais, garantindo assim que, na fase seguinte, cada caminho percorrido em $T$ tivesse no máximo dois C-nós consecutivos. Apa- 
rentemente, essa versão ficou bem mais rápida que a anterior e partimos para o desenvolvimento de uma nova versão que resolvesse o problema da planaridade. O primeiro passo nesse sentido foi deduzir como construir uma descrição combinatória plana de um grafo planar, já que tanto o artigo de Shih e Hsu quanto o artigo de Thomas omitiam essa parte. (Essa foi a parte mais complicada no desenvolvimento da implementação de SH.)

Após conseguirmos uma versão que resolvesse o problema da planaridade, partimos para os experimentos, nos baseando no programa de demonstração presente no LEDA, chamado planarity_time. Adaptamos este programa de forma a incorporar a nossa implementação de $\mathrm{SH}$ e, nas versões 4.2 e 4.3 do LEDA, notamos que as implementações de BL e de HT estavam quadráticas. (Aparentemente, na versão 4.4 do LEDA esses problemas foram solucionados.) Nos primeiros testes realizados com planarity_time, notamos que a nossa implementação de SH também estava quadrática, mas com o uso do programa gprof, conseguimos ajustar nosso programa de modo que consumisse tempo linear.

Durante essa fase de testes, tivemos a oportunidade de olhar rapidamente o artigo de Boyer e Myrvold [3] e entramos em contato com o John M. Boyer, que gentilmente nos cedeu seu código fonte em $C$, que foi adiciondo ao programa planarity_time, além de nos fornecer informações valiosas para corrigir e melhorar nossa implementação de SH. Com estas informações, eliminamos as duplicações das listas RBC e DCP associadas a C-nós ativos, através do uso do tipo bmlist (proposto por Boyer e Myrvold) e corrigimos a maneira de como era feito o percurso na PC-árvore alterada $T$ (conforme descrito na seção 4.5) de modo que C-nós inativos só sejam percorridos no caso do cálculo da lista de arcos de uma $X Y$-obstrução.

A versão final da implementação de $\mathrm{SH}$, que resolve o problema da planaridade, ficou com quatro passos básicos: cálculo dos terminais, busca por uma $X Y$-obstrução, cálculo da descrição combinatória plana para cada novo C-nó e atualização da PC-árvore alterada $T$ para a próxima iteração. Note que um dos passos da primeira versão foi dividido em: um passo para buscar uma $X Y$-obstrução e outro para atualizar $T$ para a próxima iteração, caso nenhuma $X Y$-obstrução seja detectada no passo anterior. Isso foi feito pois tanto o cálculo da lista de arcos de uma $X Y$ obstrução quanto o cálculo de uma descrição combinatória de um novo C-nó utilizam a árvore $T$ corrente, além dos valores de $b$ correntes.

Apesar da versão atual consumir tempo linear no número de vértices do grafo, conforme demonstrado neste trabalho, os resultados obtidos pelos testes realizados no capítulo 7 mostram que nossa implementação está muito lenta (principalmente quando submetemos grafos planares ao algoritmo), quando comparada às implementações presentes no LEDA. Na realidade, esta versão é um protótipo onde muito pode ser melhorado, principalmente na parte em que ocorre o cálculo de uma descrição combinatória plana para um novo C-nó e uma possível proposta de trabalho seria reescrever este programa de forma que fique "mais limpo" e "comparável" às implementações já existentes. 


\section{Índice}

\subsection{Blocos do código}

〈adiciona arcos subindo em T0 104b)

〈alguns arcos na fronteira da face externa 112b)

〈arcos do pai de um c-nó 109b)

$\langle\operatorname{arcos}$ em T0 do terminal e arcos de retorno 110)

〈atualiza rótulos b 70 〉

〈construção de um grafo 12b〉

〈constrói novo c-nó 71)

〈constrói RBC do novo c-nó 73)

〈constrói RBC partindo de um terminal 74a)

〈Critério combinatório 24〉

〈cálculo da DCP para o novo c-nó 86)

〈cálculo da DCP subindo em T 91〉

〈cálculo de terminais 62〉

〈declaração de uma PC-árvore 53〉

〈declarações 12a)

〈desenha arestas de retorno 92〉

〈emenda um RBC antigo ao novo RBC 74b)

〈encontra c-nó ativo 117)

〈enumeração dos vértices e arcos 13〉

〈função auxiliar para procurar c-nó ativo 58)

〈função principal testar planaridade 55〉

〈gera 1 122〉

〈gera 2 121)

〈gera grafos aleatórios planares 124〉 
〈gera grafos aleatórios planares + K33 126)

〈gera grafos aleatórios planares + K5 128〉

$\langle$ gera grafos maximais planares 130〉

〈gera grafos maximais planares mais uma aresta 132〉

〈group back-edges 90a)

〈inclui DCP de um c-nó antigo a DCP de um novo c-nó 96)

〈lista de arcos na fronteira da face externa 105〉

〈marca caminho em T como VISITADO 89

〈obstrução de dois no mesmo c-nó 113〉

〈obstrução de três no mesmo c-nó 111)

〈obstrução K5 109a)

〈obstrução pelo contador do c-nó 102〉

〈obstrução por T0 115〉

〈ordena arestas de retorno 94〉

〈ordena nós pelo valor de b 112a)

〈procura arco 104a)

〈procura c-nó ativo 57〉

〈procura uma obstrução 63〉

〈Programa 14〉

$\langle$ tempo de uso de CPU 120〉

〈testa caminho Q' 69)

〈teste de um c-nó 68)

$\langle$ teste partindo de um terminal 65$\rangle$ 


\section{Referências Bibliográficas}

[1] L. Auslander e S.V. Parter, On imbeddings graphs in the plane, J. Math. and Mech. 10 (1961), no. 3, 517-523. Citado na(s) página(s) [ii, 2, 4]

[2] K.S. Booth e G.S. Lueker, Testing the consecutive ones property, interval graphs, and graph planarity using PQ-tree algorithms, Journal of Computer and System Science 13 (1976), 335-379. Citado na(s) página(s) [3, 4, 25]

[3] J. Boyer e W. Myrvold, Stop minding your P's and Q's: A simplified $O(n)$ planar embedding algorithm, Procedding of the Tenth Annual ACM-SIAM Symposium on Discrete Algorithms (Baltimore, Maryland), ACM Special Interest Group on Algorithms and Computation Theory and SIAM Activity Group on Discrete Mathematics, ACM Press, January 1999, pp. 140-146. Citado na(s) página(s) [ii, 3, 4, 13, 25, 136]

[4] John M. Boyer, Corrections to the PC-tree planarity algorithm, http://pacificcoast.net/ Iightning/jboyer.htm, January 2003. Citado na(s) página(s) [135]

[5] E.R. Canfield e S.G. Williamson, The two basic linear time planarity algorithms: Are they the same?, Linear and Multilinear Algebra 26 (1990), 243-265. Citado na(s) página(s) [3]

[6] Reinhard Diestel, Graph theory, Graduate Texts in Mathematics, Springer, 1991. Citado na(s) página(s) [45]

[7] K. Mehlhorn e St. Näher, The LEDA Platform of Combinatorial and Geometric Computing, Cambridge University Press, 1999. Citado na(s) página(s) [3, 11]

[8] Shimon Even, Graph algorithms, Computer Science Press, 1979. Citado na(s) página(s) $[47,48]$ 
[9] A.J. Goldstein, An efficient and constructive algorithm for testing whether a graph can be embedded in a plane, Graph and Combinatorics Conference, Dept. Math., Princeton University, 1963, pp. 16-18. Citado na(s) página(s) [ii, 2, 4]

[10] J. Hopcroft e R. Tarjan, Efficient planarity testing, Journal of the Association for Computing Machinery 21 (1974), no. 4, 549-568. Citado na(s) página(s) [ii, 3, 4]

[11] C. Kuratowski, Sur le probleme des corbes gauches en topologie, Fundamenta Mathematicae 15 (1930), 271-283. Citado na(s) página(s) [2]

[12] A. Lempel, S. Even e I. Cederbaum, An algorithm for planarity testing of graphs, Proceddings International Symposium on Theory of Graphs (New York) (P. Rosenstiehl, ed.), Gordon and Breach, July 1967, pp. 215-232. Citado na(s) página(s) [ii, 2, 3, 4, 25, 33]

[13] K. Mehlhorn e P. Mutzel, On the embedding phase of the Hopcroft and Tarjan planarity testing algorithm, Algorithmica 16 (1996), no. 2, 233-242. Citado na(s) página(s) [3]

[14] P. Mutzel, A fast $O(n)$ embedding algorithm based on the Hopcroft-Tarjan planarity test, Tech. Report 92.107, Universität zu Köln, 1992, http://www.mpi-sb.mpg.de/ ${ }^{\sim m u t z e l / m p i r e p o r t s / p u b l i c a t i o n s . h t m l . ~ C i t a d o ~ n a(s) ~ p a ́-~}$ $\operatorname{gina}(\mathrm{s})[3]$

[15] W.-K. Shih e W.-L. Hsu, A new planarity test, Theoretical Computer Science 223 (1999), 179-191. Citado na(s) página(s) [ii, 3, 4, 25, 26, 48, 49, 135]

[16] Cormen \& Leiserson \& Rivest \& Stein, Introduction to algorithms, The MIT Press, 2001. Citado na(s) página(s) [50, 135]

[17] R.E. Tarjan, Implementation of an efficient algorithm for planarity testing of graphs, Dec. 1969. Citado na(s) página(s) [3, 25]

[18] R. Thomas, Planarity in linear time, http://www.math.gatech.edu/ thomas/, June 1997. Citado na(s) página(s) [25, 26, 135] 


\title{
Índice Remissivo
}

\author{
$(V, A), 7$ \\ $(u, v), 7$ \\ A, 7 \\ activecnode, 53 \\ $A_{G}, 7$ \\ $\operatorname{Adj}(u), 10$ \\ $E_{G}, 8$ \\ adjacentes, 8 \\ algoritmo de SH, 49 \\ ancestral, 9 \\ próprio, 9 \\ $G-V, 7$ \\ $G\left[V_{H}\right], 7$ \\ $I, 15,16$ \\ $T_{u}, 9$ \\ $V, 7$ \\ $V_{G}, 7$ \\ $X Y$-caminho, 28 \\ $X Y$-obstruções, 28 \\ resta, 8 \\ arestas de retorno, 59 \\ base, 27 \\ bloco, 9 \\ bmlist, 13 \\ bp-árvore, 9 \\ bp-número, 10 \\ $\operatorname{bp}(u), 10$ \\ $\{u v, v u\}, 8$ \\ $b(u), 54$ \\ $h(u), 54$ \\ bp-ordenação, 10 \\ C-nó, 45 \\ c-nós \\ C-nós \\ ativos, 52 \\ $m, 8$ \\ $n, 8$ \\ $u v, 7$ \\ entra, 7 \\ sai, 7 \\ tem reverso, 7 \\ C-nós \\ inativos, 52 \\ caminho, 8 \\ perimetral, 27 \\ trivial, 9 \\ caminho complementar, 27 \\ árvore, 9 \\ de blocos, 45 \\ de busca em profundidade, 9 \\ ciclo, 8 \\ facial, 19 \\ geradora, 9 \\ ciclo facial \\ determinado, 85
}


externo, 85

circuito, 8

componente, 9

contração de aresta, 33

$\operatorname{DCP}(c), 85$

descendente, 9

próprio, 9

descrição combinatória, 19

plana, 21

descrição combinatória plana alinhada, 85

desenho, 15

plano, 16

enraizada, 9

enxergar através, 28

fórmula

de Euler, 16

face, 16

externa, 16

filho, 9

real, 52

floresta, 9

grafo, 8

arcos, 7

biconexo, 9

bipartido, 9

conexo, 9

orientado, 7

planar, 16

plano, 16

simétrico, 8

vértices, 7

vazio, 7

grafo de Kuratowski, 18

grau de um vértice, 8

incidente, 8 irmãos, 9

LEC, 25

lec-ordenação, 33

listas de adjacências, 10

moldura, 26

moldura de um subgrafo, 26

P-nó, 45

pai, 9

real, 52

passeio, 8

PC-árvore

PC-árvore alterada, 52

PC-árvore, 50

ponta

final do arco, 7

inicial do arco, 7

pontas da aresta, 8

pontas do passeio, 8

predecessor, 43

problema

da lec-ordenação, 42

da nova moldura, 42

da planaridade, 24

do teste de planaridade, 23

problema do $X Y$-caminho, 42

ptritem, 53

raiz, 9

$\mathrm{RBC}(c), 50$

reverso, 7

span, 27

st-ordenação, 33

subárvore, 9

subdivisão, 18

de aresta, 18

subgrafo 
induzido, 7

subgrafo orientado, 7

sucessor, 43

tamanho de $G, 8$

terminal, 47, 59

vértice

de corte, 9

vértices

internos do passeio, 8

vizinhos, 7 